\title{
Development of an ultrafast low-energy electron diffraction setup
}

\author{
DissERTATION \\ zur Erlangung des mathematisch-naturwissenschaftlichen \\ Doktorgrades \\ "Doctor rerum naturalium" \\ der Georg-August-Universität Göttingen \\ im Promotionsprogramm ProPhys \\ der Georg-August University School of Science (GAUSS) \\ vorlegt von \\ Herrn Dipl.-Phys. Max Gulde \\ aus Reutlingen
}

Göttingen, 2014 


\section{Betreuungsausschuss}

Prof. Dr. Claus Ropers, IV. Physikalisches Institut

Prof. Dr. Tim Salditt, Institut für Röntgenphysik

\section{Mitglieder der Prüfungskommission}

Referent Prof. Dr. Claus Ropers, IV. Physikalisches Institut

Korreferent Prof. Dr. Tim Salditt, Institut für Röntgenphysik

2. Korreferent Prof. Dr. Klaus Sokolowski-Tinten, Universität Duisburg-Essen

Prof. Dr. Wolfram Kollatschny, Institut für Astrophysik

Prof. Dr. Hans-Ulrich Krebs, Institut für Materialphysik

Prof. Dr. Marcus Müller, Institut für Theoretische Physik

Prof. Dr. Holger Stark, Max-Planck-Institut für Biophysikalische Chemie

Tag der mündlichen Prüfung: 15. Oktober 2014 


\section{Abstract}

Quasi two-dimensional systems such as surfaces and atomically thin films can exhibit drastically different properties relative to the material's bulk, including complex phases and transitions only observable in reduced dimensions. However, while methods for the structural and electronic investigation of bulk media with ultrahigh spatio-temporal resolution have been available for some time, there is a striking lack of methods for resolving structural dynamics at surfaces.

Here, the development of an ultrafast low-energy electron diffraction setup is presented, offering a temporal resolution of a few picoseconds in combination with monolayer structural sensitivity. In particular, a detailed account is given on the defining beam properties of the electron source, based on a nonlinearly driven nanometric photocathode. The emitter parameters within an electrostatic lens assembly are studied by means of a finite element approach. In particular, the optimal operation regime as well as achievable temporal resolution are determined. A prototype emitter comparable to the one used in the simulation is designed, characterized and applied within an ultrafast low-energy diffraction experiment. Specifically, the superstructure dynamics of an ultrathin bilayer of polymer crystallites adsorbed on free-standing graphene are investigated upon strong out-of-equilibrium excitation. Different processes in the superstructure relaxation are identified together with their respective timescales between 40 and 300 ps, including the energy transfer from the graphene to the polymer, the loss of crystalline order and the formation of extended amorphous components. The findings are subsequently discussed in view of an ultrafast melting of the superstructure. To conclude, the contribution of the approach to time-resolved surface science is discussed and an outlook is given in terms of future systems to investigate and further developments of the apparatus.

\section{Keywords}

Ultrafast Low-Energy Electron Diffraction, Electron Pulses, Surface Science, Structural Analysis, Superstructure Dynamics, Ultrathin Polymer Film, Graphene, PMMA 



\section{Contents}

Abstract

Contents $\quad$ v

1 Introduction 1

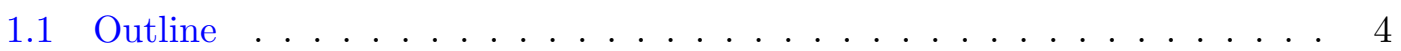

2 Methods and concepts $\quad 7$

2.1 An introduction to LEED . . . . . . . . . . . . . . . . . . 7

2.2 Diffraction pattern formation in two dimensions . . . . . . . . . . . . . 10

2.3 Graphene and graphene technology . . . . . . . . . . . . . . . . . 17

3 Aspects of ultrafast LEED $\quad \mathbf{2 3}$

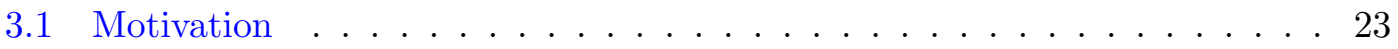

3.2 Ultrahigh temporal resolution via pump-probe . . . . . . . . . . . 26

3.3 From continuous to pulsed electron sources . . . . . . . . . . . . . 28

3.4 Beam properties of tip-based electron sources . . . . . . . . . . . . . . . 31

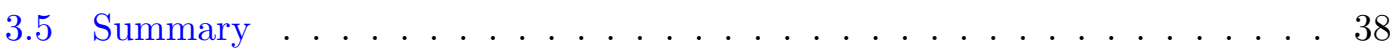

4 Numerical analysis of a tip-based ultrafast electron gun 41

4.1 Finite element simulation . . . . . . . . . . . . . . . . 41

4.2 The suppressor / extractor unit . . . . . . . . . . . . . . . . 44

4.3 The lens: pulse duration, focusability, and total current . . . . . . . . . 47

4.4 Energy dispersion . . . . . . . . . . . . . . . . . 50

4.5 Summary and discussion . . . . . . . . . . . . . . . . . . 51

5 Experimental analysis of a tip-based ultrafast electron gun $\quad \mathbf{5 5}$

5.1 Tip preparation . . . . . . . . . . . . . . 55

5.2 ULEED experimental setup . . . . . . . . . . . . . . . . 57

5.3 Experimental characterization of the ultrafast electron gun . . . . . . . 59

5.4 Summary and discussion . . . . . . . . . . . . . . . . . . 67

6 Ultrafast PMMA superstructure dynamics on free-standing graphene $\mathbf{7 5}$

6.1 Choice of a sample system . . . . . . . . . . . . . . . . 75

6.2 Bilayer characterization in the ULEED setup . . . . . . . . . . . . . 79

6.3 Ultrafast relaxation dynamics of the PMMA superstructure . . . . . . . . 91

6.4 Physical picture and discussion . . . . . . . . . . . . . . . . . . . . . . . . . . . . . . . . . . . . .

6.5 Summary . . . . . . . . . . . . . . . . . 106 
7 Conclusions $\quad 109$

7.1 Summary . . . . . . . . . . . . . . . . . . . . . 109

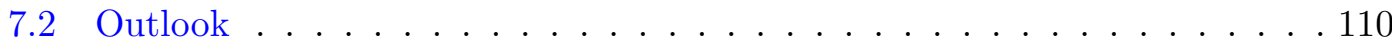

$\begin{array}{lr}\text { A Methods and Calculations } & 117\end{array}$

A.1 Analytical model for pulse durations . . . . . . . . . . . . . . . . . . . 117

A.2 The electron inelastic mean-free-path . . . . . . . . . . . . . . . . 120

A.3 Finite element method . . . . . . . . . . . . . . . . . . . . . 121

A.4 Data normalization and fitting . . . . . . . . . . . . . . 123

A.5 Separation of diffraction spot and disc contributions . . . . . . . . . . 123

A.6 Reproducibility of the measurements . . . . . . . . . . . . . . . . . . . 124

$\begin{array}{lr}\text { B Sample characterization } & 127\end{array}$

B.1 Graphene characterization . . . . . . . . . . . . . . . . . . . 127

B.2 Formation time of the folded-chain crystallites . . . . . . . . . . . . . 129

B.3 Thermal resistance of the superstructure . . . . . . . . . . . . . . 130

B.4 Superstructure degradation . . . . . . . . . . . . . . . . . . 131

$\begin{array}{ll}\mathrm{C} \text { Abbreviations and symbols } & \mathbf{1 3 5}\end{array}$

C.1 Abbreviations . . . . . . . . . . . . . . . . . . . 135

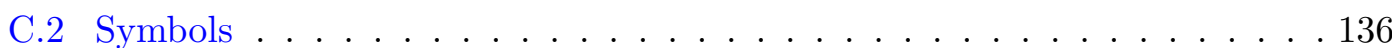

$\begin{array}{lr}\text { Bibliography } & 139\end{array}$

$\begin{array}{lc}\text { Publications and Conferences } & 173\end{array}$

$\begin{array}{ll}\text { Acknowledgments } & 175\end{array}$ 


\section{Chapter 1}

\section{Introduction}

The outermost atomic layers in a solid are generally referred to as its surface. Whenever a physical or chemical interaction occurs between two objects, the result of this interaction is subject to the individual properties of the surfaces in contact with each other. Interestingly, even though the surface is in principle made up from the same constituents as the material's volume (its bulk), it often exhibits drastically different behavior in terms of its structural or electronic properties [1]. The reason for this discrepancy originates in the symmetry breaking at the surface with the solid on the one side and, e.g., vacuum on the other, leading to a strong coupling of electronic and lattice systems.

The study of surfaces and their physical and chemical properties constitutes "surface science". One of its main objectives is to relate the microscopic structure of a given surface to the material's macroscopic properties. Although optical, electrical, magnetic and chemical investigations have been performed systematically for a long time, quantitative results on an atomistic level became only available with relatively recent technological advances. In particular, ultrahigh vacuum technology, the development of suitable detection systems, and the appearance of digital computers have propelled experimental and theoretical progress $[2,3]$. This has resulted in a rapid increase in the number of available surface sensitive techniques, reaching from atomic force microscopy (AFM) [4] and low-energy electron diffraction (LEED) [5, 6] to scanning electron microscopy (SEM) [7] and scanning tunneling microscopy (STM) [8] to (grazing incidence) X-ray diffraction (XRD) [9]. 
For structural surface analysis, LEED has an outstanding role. In particular, it combines extremely high surface sensitivity (under certain conditions down to a fraction of a monolayer) with the possibility of atomic structure determination, a direct and fast interpretation of symmetry information, and a robust (but involved) theoretical background, all in an overall low-cost approach. Additionally, Auger spectroscopy can be straight-forwardly implemented within a LEED setup to retrieve chemical information about the surface. These properties make LEED one of the most prominent structural surface techniques of today.

Paralleling the technological developments, new methods in surface science have quickly emerged, which are yet again motivating new applications. These applications include corrosion and wear protection by means of self-assembling monolayers [10], superconductorsemiconductor interfaces as a basis for quantum computing [11-13], catalysis in surface chemistry, e.g., in view of energy storage and production [14-16] and giant magnetoresistance for the development of non-volatile information storage devices [17], to name but a few. Moreover, the recent possibility to atomically tailor materials, such as graphene heterostructures and composite materials, has additionally accelerated the trend of surface/interface enlargement with respect to volume [18-21], making surfacesensitive studies increasingly important in view of current scientific and technological advances. Generally speaking, surface science has become a major field of material science.

In the context of the wealth of observed surface structures, reflected for example in the existence of phases and phase transitions limited to two-dimensional systems [22-25], an equally high degree of diversity is to be expected from structural dynamics at surfaces. This assumption is substantiated, for example, by recent experimental and theoretical studies on surface premelting [26, 27], the investigation of negative thermal expansion coefficients at surfaces [28] and the observation of surface charge density waves (sCDW) [29].

In stark contrast to the richness of explorable systems, time-resolved methods to actually follow structural changes during physical and chemical processes at surfaces are sparse. On the other hand, ultrafast time resolution ${ }^{1}$ has been successfully implemented

\footnotetext{
${ }^{1}$ Here, the term ultrafast is used for timescales below one nanosecond.
} 
in a number of systems for bulk analysis, including time-resolved electron [30] and $\mathrm{x}$ ray spectroscopy [31, 32], ultrafast electron diffraction (UED) [33-35] and microscopy [36-38], time-resolved second harmonic and sum frequency spectroscopy [39], as well as ultrafast x-ray crystallography $[40,41]$. Whereas some of these techniques such as photoelectron spectroscopy can be applied to the probing of the electronic structural evolution at surfaces [42], time-resolved atomic structural information of surfaces is challenging to obtain.

So far, for the investigation of a surface's atomic structure, mainly x-ray or electron-based techniques are employed. While x-rays offer supreme temporal resolution combined with high monochromaticity, electrons are less damaging to specimens, the technology of electron imaging is well-developed, electron sources are brighter and their interaction with matter stronger [43].

Depending on the probe, high surface sensitivity can be in principle achieved by using either large angles of incidence, as in XRD and reflection high-energy electron diffraction (RHEED) [44], or low kinetic electron energies. Large angles of incidence, however, have the disadvantage to be strongly dependent on the surface morphology, which makes a quantitative analysis difficult. Additionally, a grazing-incident geometry not only results in large probing areas on the sample, hence making a localized probing challenging, but also limits the achievable temporal resolution of the technique, necessitating a tilted wave front setup [45]. On the other hand, when using low-energy electrons, space charge effects and dispersive broadening strongly decrease the temporal resolution [46], so far preventing the development of time-resolved LEED with sub-nanosecond resolution [47].

Only recently, the availability of table-top ultrafast laser sources in combination with nano-engineered photocathodes has opened up a new gateway to potentially develop low-energy electron sources capable of ultrafast probing [48-51]. In particular, nonlinearly driven, nanometrically sharp needle emitters are suggested to minimize broadening effects [52-54].

In the experimental work presented in this thesis, an ultrafast LEED (ULEED) setup in transmission is developed, featuring a temporal resolution of few picoseconds at low electron energies. Particularly, this setup represents the first application of a tip-based emitter in a diffraction experiment. To demonstrate the feasibility of the approach, free-standing graphene is chosen as a prototype material, offering a well-defined atomic 
structure in combination with high mechanical and thermal stability. Specifically, the structural relaxation dynamics of an ultrathin polymer superstructure, adsorbed on the graphene substrate and excited to a highly out-of-equilibrium state, is investigated. The characteristic time scales of the superstructure evolution are subsequently identified and a physical picture is derived from the observations.

This novel tool in time-resolved material science carries the potential to allow for the investigation of atomic scale structural dynamics at surfaces and thin films with ultrahigh temporal precision.

\subsection{Outline}

The organization of this thesis is as follows: In Chap. 2, the reader is introduced to the theoretical concepts and methods commonly employed in surface science. This includes a brief description of the LEED concept, followed by basic diffraction theory and a description of the materials constituting the sample system of Chap. 6 .

The main body of the thesis is divided into two parts. The first deals with the development of a new electron source for time resolved surface studies and its subsequent theoretical and experimental characterization (Chap. 3, 4, and 5). In contrast, the second part displays the application of the technique to resolve the ultrafast dynamics of a first sample system (Chap. 6).

More specifically, Chap. 3 gives a brief overview of the current state of the art in ultrafast surface science. Subsequently, the feasibility of nonlinearly driven nanometric electron sources in terms of an application in an ultrafast LEED setup is investigated. The characterizing source properties, such as coherence, brightness and temporal resolution, are calculated theoretically.

In the subsequent Chap. 4, a finite element method (FEM) is employed to further investigate the performance of an electron gun based on nanoscale photoemission. Particularly, the optimal electron source parameters are determined for highest brightness and temporal resolution.

Chapter 5 is the experimental analogue to its predecessor, investigating the properties of a prototype electron source within a setup for low-energy electron diffraction. The 
theoretically and experimentally found quantities are compared and discussed. The chapter closes with an outlook on potential developments of the tip-based source to further increase its temporal and spatial resolution.

The application of the setup to resolve the structural evolution of a sample system is described in Chap. 6. Specifically, the relaxation dynamics of a polymer superstructure adsorbed on graphene, are investigated. The governing time scales of different processes are determined and a physical picture based on the observations is provided.

In the last chapter, a brief summary of the major aspects of the thesis is given. In particular, Chap. 7 concludes with an outlook on the ongoing molecular dynamics simulations, prospective systems to study in the future, as well as the further development in terms of ULEED.

There are two suggested pathways of reading. Whereas the scientist new to the field of time-resolved material science may just follow the thesis in the given order, a reader with a background in (electron) diffraction is suggested to skip Chap. 2. Furthermore, those parts of Chap. 3, which are introducing the concepts of brightness, emittance and coherence, can be equally omitted. The relevant observation in terms of electron source performance are repeated at the end of the chapter. For the remaining thesis, the given order can be followed. 



\section{Chapter 2}

\section{Methods and concepts}

This chapter offers an introduction to the theoretical and experimental methods, which are important in the framework of this thesis. First, the concept of low-energy electron diffraction as a tool for surface structural investigations is outlined. Specifically, after a brief historical overview on the development of LEED in combination with its key features and challenges (Sec. 2.1.1), the typical experimental setup is illustrated (Sec. 2.1.2). In the following section, the basics of surface-diffraction theory are presented in view of the analysis performed in the time-resolved surface study of Chap. 6 (Sec. 2.2). For this purpose, the materials comprising the sample system are introduced (Sec. 2.3).

\subsection{An introduction to LEED}

\subsubsection{LEED historical context and key aspects}

Low-energy electron diffraction is a technique for the structural investigation of crystalline surfaces based on the diffraction of electrons with low kinetic energy. The origins of LEED go back to the 1920s, when C. J. Davisson and L. H. Germer directed a beam of monochromatic, slow electrons at a nickel single-crystal under vacuum conditions [5]. They found that the recorded angular intensity pattern of the scattered electrons was in agreement with the concept of diffraction of wave-like electrons, as had recently been proposed by L. de Broglie [55]. At the same time, G. P. Thomson independently made similar observations with faster electrons in a backscattering as well as in a transmission geometry $[56,57]$. 
These findings mark the beginning of modern electron diffraction experiments such as LEED, which then eventually developed into the powerful tool in surface crystallography it is today. With respect to the technique of X-ray diffraction from crystals, which was already well-established at that time $^{1}$, the use of electrons presented a few important advantages:

- X-rays are scattered relatively weakly by matter, resulting in large penetration depths, making electron-based methods far more sensitive to the surface structure.

- The energy of electrons can be easily adapted to fit the investigated sample, which is a far more involved task for most X-ray sources.

- Electronic and magnetic lens systems allow for a straight-forward manipulation and tailoring of the electron beam, while X-ray optics are challenging to produce.

- For the typical electron energies used for highest surface sensitivity, the electron wavelengths are comparable to the lattice spacing of most crystals, leading to large scattering angles.

Despite these advantages, the subsequent development of LEED came to an untimely end only few years later, owning largely to difficulties in meeting the requirements associated with the experimental setup. Specifically, high surface crystallinity as well as cleanliness of the sample both necessitated operation under ultrahigh vacuum conditions, which were not easily achievable at the time. Additionally, the lack of spatiallyresolved detector systems and computational resources made LEED recordings arduous and time-consuming.

Only with the availability of suitable vacuum, electron sources and detection techniques in the 1960s, LEED achieved a wide acceptance as a surface probing tool within the scientific community. These advantages also in turn led to the development of the theoretical methods based on multiple scattering to allow for a quantitative understanding of the recorded data. With the technological requirements met, LEED rapidly became one of the standard methods for structure determination [59]. Nowadays, besides qualitative investigations on the symmetry and periodicity of a surface, the analysis of I-V curves and spot profiles in combination with robust numerical methods enables scientists to

\footnotetext{
${ }^{1}$ The first quantitative X-ray bulk structural analysis was performed in 1913, only one year after the initial prove of X-ray diffraction [58].
} 
obtain detailed information about crystal defects as well as the precise atomic arrangement within a surface unit cell. Moreover, LEED setups are routinely coupled to Auger electron spectroscopy (AES), additionally yielding information about the chemical composition of surface contaminants [60].

\subsubsection{Experimental Setup}

LEED investigates the surface structure of a given crystalline sample by recording the scattered diffraction orders. Commonly, as shown in Fig. 2.1A, a collimated electron beam for sample probing is generated within a thermionic electron gun. The latter comprises a heated cathode filament and an electrostatic lens system, whereby LEED gun currents are typical in the range of $10^{-4}$ and $10^{-8} \mathrm{~A}$ [61]. The emitted electrons are then backscattered by the sample and subsequently recorded on a spherical cap-shaped fluorescent phosphor screen. Since only the elastically scattered electrons carry the wanted structural information [62], an energy filter is employed to deflect any inelastically scattered electrons. The energy filter is most commonly made from a series of fine metal grids with the inner ones set to a retarding electrostatic potential just below the initial electron energy, allowing only elastically scattered electrons to pass.
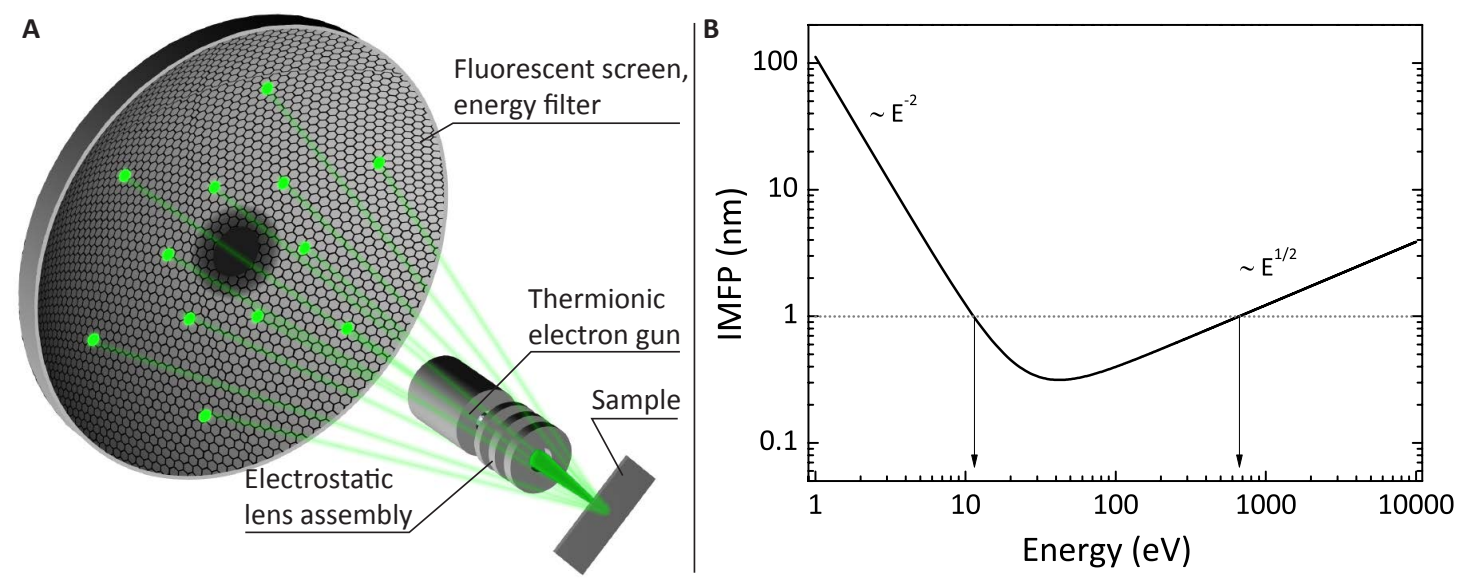

FIGURE 2.1: Sketch of LEED principle and IMFP computation. A: Electrons generated by a thermal emitter are focused on the sample by an electrostatic lens system. The (elastically) backscattered electrons are then recorded on a fluorescent screen after passing an energy filter (usually both shaped as a spherical cap). B: Universal curve of IMFP for electrons in a solid [63]. Energy range with highest surface sensitivity below $1 \mathrm{~nm}$ indicated by arrows.

Due to the use of low-energy electrons, LEED is extremely surface sensitive and has to be carried out under ultrahigh vacuum conditions to avoid sample contamination, e.g. through oxidation or adsorbed molecules. A plot of the inelastic mean-free-path 
(IMFP) as a function of the kinetic electron energy is shown in Fig. 2.1B [63] (for computation, see App. A.2). The minimal IMFP for most materials, including graphite, is found at electron energies of about $50 \mathrm{eV}$. Nevertheless, very high surface sensitivity with an IMFP below $1 \mathrm{~nm}$ is generally achieved for energies between $10 \mathrm{eV}$ and $700 \mathrm{eV}$. This sensitivity stems from the collective excitation of vibrational lattice (phonons) and electron (plasmons) modes with energies between a few to some tens of electron volts above the Fermi level [6]. These excitations significantly reduce the typical penetration depth of impinging electrons by removing a substantial fraction of their initial kinetic energy.

Depending on the investigated quantity, LEED is operated in the following ways:

1. For a qualitative analysis of the surface structure in terms of lattice spacing, periodicity and symmetry, merely a reciprocal scale has to be determined to access these parameters directly. This can either be done by knowledge of the experiment's exact geometry or a reference diffraction pattern.

2. For a more quantitative analysis, the diffraction spot profile can be used to determine deviations from the ideal order, including the domain size (spot width) and the existence of steps, facets or surface defects (shape) [64, 65].

3. In order to extract information about the precise internal unit cell structure, the diffraction spot intensity is recorded as a function of electron energy (so-called $I-V$ curves) [66-68]. An iterative approach based on the comparison with a theoretical model then leads to the atomic arrangement within the unit cell.

The combination of these analytical capabilities makes LEED into a versatile tool for detailed structural surface characterization.

\subsection{Diffraction pattern formation in two dimensions}

Diffraction can be seen as a result of the interaction between an incoming, periodic wave field and a likewise periodic array of scattering centers [6]. At large enough wave numbers $\mathbf{k}_{0}$ of the incoming wave, the amplitudes of scattered waves are in-phase along certain directions (diffraction condition), resulting in an observable intensity on the detector. 
Normally, not only one but a larger number of conditions along different scattering directions are met simultaneously, resulting in the appearance of multiple beams and the so-called diffraction pattern.

To obtain a mathematical description of the process, we are assuming a monochromatic incoming plane wave as well as an infinite periodicity of the sample. This requirement is usually an adequate assumption in two dimensions with at most a few layers in the direction perpendicular to the surface, as well as an in-plane periodicity, which is limited only by either the domain size of the sample or the coherently probed area.

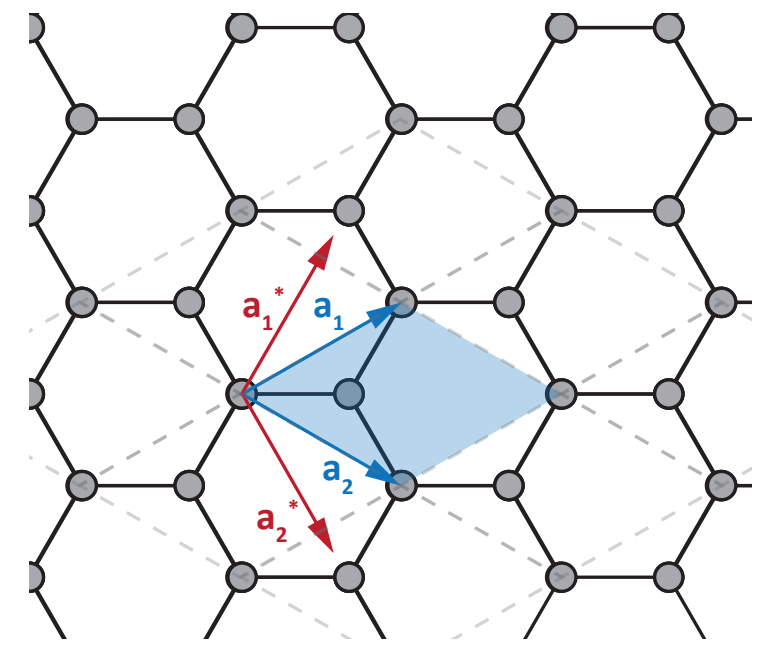

Figure 2.2: Sketch of a single unit cell (blue) with lattice vectors $\mathbf{a}_{\mathbf{1}}$ and $\mathbf{a}_{\mathbf{2}}$ in case of a hexagonally symmetric surface atom arrangement. Dashed areas denote repeating unit cells. Reciprocal lattice vectors displayed in red.

The surface periodicity is given in terms of a lattice. A lattice is defined as the simplest arrangement of points which follow the fundamental periodicity of the crystal [6]. Individual mesh cells of a lattice are called unit cells and carry the relationship between the lattice and the actual atomic positions (Fig. 2.2). Unit cells are chosen according to be the smallest possible repeating unit to fully describe the crystal structure. Depending on the actual atomic arrangement, different choices of unit cells are often possible to describe the same structure. A real space lattice is described by a set of two linearly independent lattice vectors defining the boundaries of the unit cell.

The above described diffraction condition, namely that a net flux of scattered waves is recorded along those directions, in which constructive interference occurs, is called the Laue condition and can be written as (Fig. 2.3)

$$
a\left(\sin \Theta_{n}-\sin \Theta_{0}\right)=n \lambda,
$$


where $\Theta_{0}$ and $\Theta_{n}$ are the angles of the incident and scattered waves, respectively, $n \lambda$ an integer multiple of the wavelength of the incoming wave, and $a=|\mathbf{a}|$ the lattice parameter.

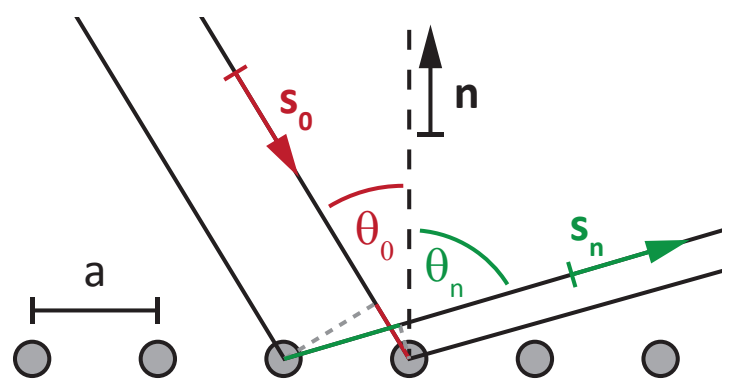

FIGURE 2.3: Sketch of diffraction from a one-dimensional array of scatterers. Dashed black line denotes "surface" normal with unit vector $\mathbf{n}$. Incoming (outgoing) wave vector labeled $\mathbf{s}_{0}\left(\mathbf{s}_{n}\right)$. Red and green lines denote path length contributions $\left(a \sin \Theta_{0}\right)$ and $\left(a \sin \Theta_{n}\right)$, respectively. Lattice parameter: a.

When written in terms of the unit vectors $\mathbf{s}_{0}$ and $\mathbf{s}_{n}$ of the incident and scattered beam, respectively, this expression becomes

$$
\mathbf{a}\left(\mathbf{s}_{n}-\mathbf{s}_{0}\right)=\mathbf{a} \Delta \mathbf{s}=n \lambda .
$$

Hence, the diffracted beam is determined by the normalized path length difference $\Delta \mathbf{s}$, which is given by integer multiples of $\lambda /|\mathbf{a}|$, a quantity proportional to the reciprocal lattice constant. Specifically, the reciprocal lattice vectors, $\mathbf{a}_{i}^{*}$, are defined in terms of the real space lattice vectors, $\mathbf{a}_{i}$, by the following relationship [1]:

$$
\mathbf{a}_{i}^{*}=2 \pi \frac{\mathbf{a}_{j} \times \mathbf{n}}{\left|\mathbf{a}_{i} \times \mathbf{a}_{j}\right|}
$$

In Eq. 2.3, $\mathbf{n}$ is the unit vector normal to the surface as depicted in Fig. 2.3. The relationship between real and reciprocal lattice vectors is often also expressed by $\mathbf{a}_{i}^{*} \mathbf{a}_{j}=$ $2 \pi \delta_{i j}$ leading to the pairwise perpendicular vectors illustrated in Fig. 2.2.

Any general translation vector, relating two reciprocal lattice points, therefore takes the form

$$
\mathbf{g}_{h k}=h \mathbf{a}_{1}^{*}+k \mathbf{a}_{2}^{*}=\Delta \mathbf{s} / \lambda .
$$

The integers $h$ and $k$ are called Miller indices. 


\subsubsection{Ewald construction}

The above-mentioned diffraction criteria can be visualized by a geometrical construction called the Ewald construction. Before doing so, we will briefly assess the impact of twodimensionality on the diffraction process.

From Eq. 2.3 one can see that whenever a real space lattice vector is increased in length, the corresponding reciprocal vector is decreased accordingly. Considering an isolated two-dimensional system, the lattice vector normal to the surface is infinitely stretched, hence the respective reciprocal lattice vector becomes infinitesimally small. This means that parallel to this direction, interference effects are eliminated, allowing the beam to be observed at all angles and energies. In the Ewald construction, this is expressed by the existence of reciprocal lattice rods rather than points for the direction normal to the surface (Fig. 2.4). As a consequence, a diffraction pattern of a quasi-two-dimensional system is seen at nearly any incident angle and energy.

A

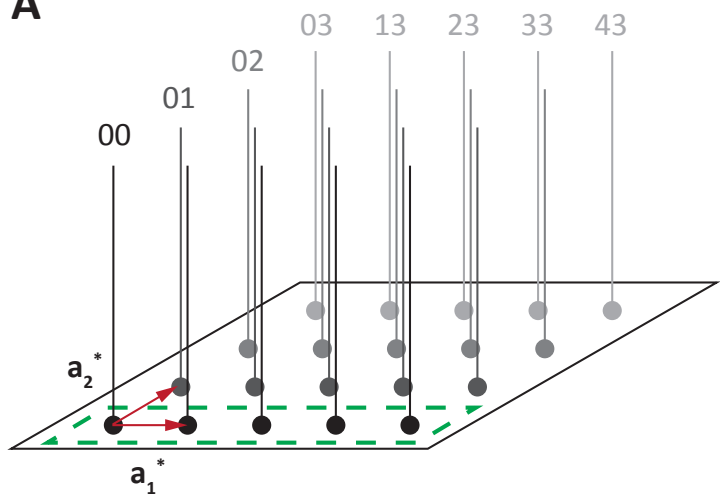

B

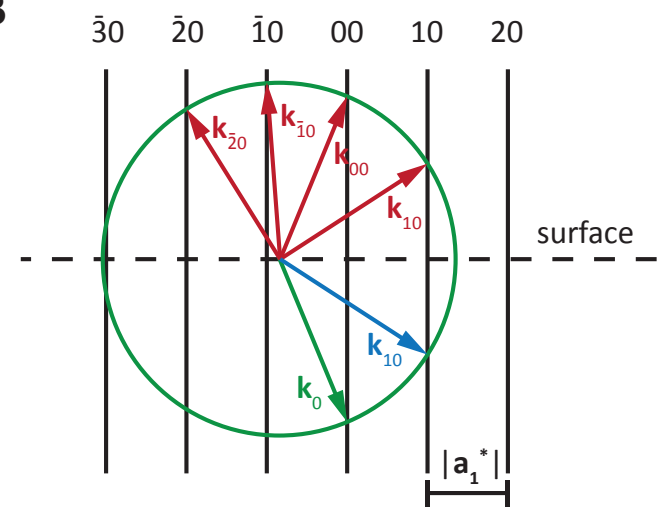

FiguRe 2.4: Ewald construction. A: Reciprocal space of a periodic surface with rods instead of points perpendicular to the surface. Numbers above rods: Miller indices. Red arrows: reciprocal unit vectors. Green rectangle denotes single row depicted in B.

B: Two-dimensional Ewald sphere. Incoming wave vector $\mathbf{k}_{0}$ in green, back-scattered (forward-scattered) wave vectors $\mathbf{k}_{h k}$ in red (blue) with $h$ and $k$ Miller indices.

In Fig. 2.4B, the Ewald sphere (in two dimensions) is shown for a single row of the surface reciprocal lattice depicted in Fig. 2.4A. The incident wave vector $\mathbf{k}_{0}=2 \pi / \lambda$ (green arrow) impinges on the sample surface and is scattered (red arrows). As stated earlier (Sec. 2.1.2), we are only concerned with the elastically scattered waves, since they carry the structural information of the sample system. If the scattered beams do not lose energy, they must have the same length as the incident wave vector, thus lying on a circle (sphere in three dimensions). Hence, the intersections of the circle of radius $\left|\mathbf{k}_{0}\right|$ 
with the lattice rods fulfill both the condition of energy conservation as well as the Laue diffraction condition.

The direct reflection from the incident beam is labeled $\mathbf{k}_{00}$ and usually has to be blocked in order to observe the other, less intense diffracted beams. In the case of very thin samples or high electron energies, not only reflected, but also transmitted beams will be seen. Notably and in stark contrast to bulk diffraction, diffraction patterns will not only be observable for certain energy-angle relations, but basically for any chosen configuration, as long as the Ewald sphere's diameter is larger than the reciprocal lattice constant.

\subsubsection{Overlayers and domains}

Based on the concept of the Ewald sphere, the total number of diffracted beams with their respective scattering angles can be determined for a given energy in combination with the knowledge of the reciprocal lattice. While the bulk structure of crystals is generally well known from x-ray crystallography, structural deviations of the surface from the bulk are quite common. Typical deviations may stem from surface reconstructions due to symmetry breaking and subsequent energy minimization or from the adsorption of molecules [6, 69]. This individual surface reconstruction will hereby strongly depend on the bulk structure, the type of molecules and bonds involved, as well as environmental factors such as temperature and pressure.

In the most general case, the lattice vectors of the overlayer or superstructure can be expressed in terms of the lattice vectors of the primary lattice [6]:

$$
\begin{aligned}
& \mathbf{b}_{1}=m_{11} \mathbf{a}_{1}+m_{12} \mathbf{a}_{2}, \\
& \mathbf{b}_{2}=m_{21} \mathbf{a}_{1}+m_{22} \mathbf{a}_{2} .
\end{aligned}
$$

This can be rewritten in matrix notation as

$$
\mathbf{b}=\left(\begin{array}{l}
\mathbf{b}_{1} \\
\mathbf{b}_{2}
\end{array}\right)=\left(\begin{array}{ll}
m_{11} & m_{12} \\
m_{21} & m_{22}
\end{array}\right)\left(\begin{array}{l}
\mathbf{a}_{1} \\
\mathbf{a}_{2}
\end{array}\right)=M \mathbf{a} .
$$


Similarly, a relationship between the reciprocal lattices can be established

$$
\mathbf{b}^{*}=M^{*} \mathbf{a}^{*}
$$

where the matrices $M$ and $M^{*}$ are related by [6]

$$
\left(\begin{array}{ll}
m_{11} & m_{12} \\
m_{21} & m_{22}
\end{array}\right)=\frac{1}{\operatorname{det} M^{*}}\left(\begin{array}{cc}
m_{22}^{*} & -m_{21}^{*} \\
-m_{12}^{*} & m_{11 *}
\end{array}\right) \Leftrightarrow M\left(M^{*}\right)^{T}=2 \pi \mathbb{1},
$$

with $\mathbb{1}$ representing the identity matrix in two dimensions. Once the reciprocal lattice vectors of the superstructure are extracted from the diffraction pattern, its real space lattice vectors can in principle be computed. Nevertheless, this will only yield the periodicity of the overlayer, but not the information about its atomic arrangement or the registration to the surface. For an identification of the atom's positions within the unit cell, additional information needs to be included, e.g., from measurements of the I-V curves of the superstructure spots or by prior knowledge about preferred bonding sites of an adsorbate.

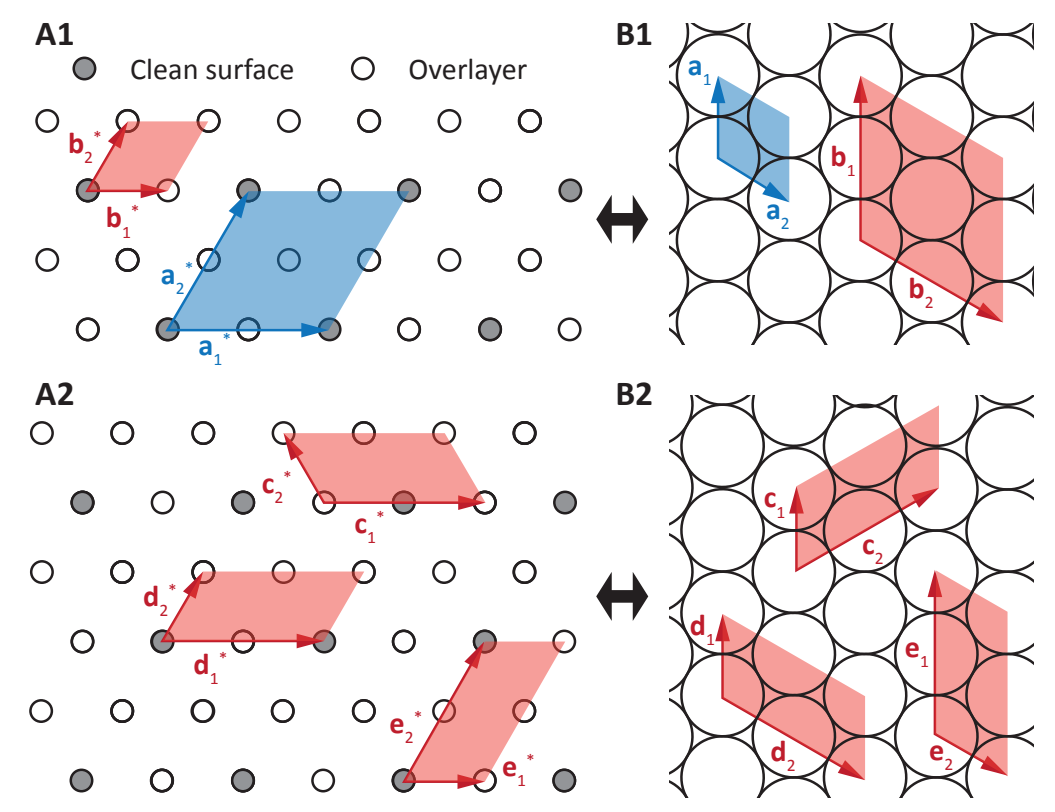

FIGURE 2.5: Exemplary ambiguity in real-space superstructure lattice reconstruction. Clean surface (overlayer) unit cell(s) in blue (red). Top panel: Interpretation as a single $2 \times 2$ overlayer structure. Bottom panel: Interpretation as a superposition of three $2 \times 1$ overlayer domains. Both real space superstructures shown in B1 and B2 result in the same diffraction pattern depicted in $\mathbf{A} \mathbf{1}$ and $\mathbf{A 2}$.

Frequently, more than one orientation of the superstructure is abundant. Such regions, which are comprising a certain orientation of the overlayer, are called domains or islands 
in case of very small coverage. Depending on the characteristic length scale of the domains, the probed sample area, and the coherence length of the source relative to each other, different results in term of observed diffraction patterns may be expected. When the domain size is comparable to the investigated sample area, the probe beam can in principle be scanned over the surface, yielding different diffraction patterns depending on the underlying domain orientation. In contrast, a domain size well below the size of the probed region leads to two distinguishable cases, depending on the ratio between coherence length $l_{c}$ and domain size $d$ :

1. $l_{c}<d$ : A superposition of diffraction patterns from different domain orientations.

2. $l_{c}>d$ : An interference between the diffraction signals from different domains.

Generally, the existence of domains can result in an additional ambiguity when interpreting the structure of the overlayer (see Fig. 2.5). In the diffraction pattern shown in Fig. 2.5A1, the clean surface (full circle) and overlayer (empty circle) spots have been identified. The overlayer is interpreted as a $2 \times 2$ superstructure (red unit cell), which translates to the real-space periodicity shown in Fig. 2.5B1. Alternatively, the same diffraction image (Fig. 2.5A2) can be understood as the superposition of three overlayer domain types as indicated in the real-space image in Fig. 2.5B2.

However, there exist several options for resolving this ambiguity in the interpretation of the diffraction patterns. For instance, the sample could be prepared in a way to allow only certain domain orientations, e.g., by the introduction of step defects [70]. Similarly, a piecewise scanning of small sample areas could reveal the abundance of domains.

When taking the diffraction pattern from surfaces or thin films with more than one atomic layer, the scattering from successive crystal planes has to be taken into account. In case of a rationally related overlayer, the lattice vectors can be expressed via Eq. 2.5 and 2.6.

An incoming beam $\mathbf{s}_{0}$ (see Fig. 2.3) then produces a series of diffracted beams following the relation given in Eq. 2.4:

$$
\mathbf{s}_{0}-\mathbf{s}_{h k}=\lambda\left(h \mathbf{b}_{1}^{*}+k \mathbf{b}_{2}^{*}\right)
$$


This beam is now incident upon another lattice plane, resulting in a new set of beams

$$
\begin{aligned}
\mathbf{s}_{h k}-\mathbf{s}_{f g} & =\lambda\left(f \mathbf{a}_{1}^{*}+g \mathbf{a}_{2}^{*}\right), \text { hence } \\
\mathbf{s}_{0}-\mathbf{s}_{f g} & =\lambda\left(\left(f+h m_{11}^{*}+k m_{21}^{*}\right) \mathbf{a}_{1}^{*}+\left(g+h m_{12}^{*}+k m_{22}^{*}\right) \mathbf{a}_{2}^{*}\right)
\end{aligned}
$$

with $f, g, h, k, m_{x y}^{*}$ integers. Therefore, the beams $\mathbf{s}_{f g}$ must correspond to the same set of angles as the $\mathbf{s}_{h k}$ set. This also means that no new diffraction beams from scattering of successive planes will be introduced and multiple scattering in this case only shifts the intensities between diffraction spots.

\subsection{Graphene and graphene technology}

Whereas surfaces and in particular atomically thin superstructures can be seen as quasitwo-dimensional systems, free-standing $2 \mathrm{D}$ materials have not been available until very recently [71]. And even though there is now a vast number of monolayer systems available, including various types of oxides (e.g. BSCCO ("bisko"), $\mathrm{Bi}_{2} \mathrm{Sr}_{2} \mathrm{Ca}_{n-1} \mathrm{Cu}_{n} \mathrm{O}_{2 n+4+x}$ ) or chalcogenides (e.g. molybdenum disulfide, $\mathrm{MoS}_{2}$ ), the material that continues to attract the most attention is graphene, a single crystalline sheet of carbon atoms [18].

One reason for the popularity of graphene as a research material are its outstanding electronic and mechanical properties as a consequence of its low dimensionality in combination with its atomic and electronic structure [72]. Specifically, the extremely high carrier mobility of graphene, even at elevated temperatures, motivates significant efforts in the field of graphene-based electronics. This includes, for example, the development of single-electron, high frequency transistors operating ballistically at room temperature [73-76] as well as devices for electromagnetic interference shielding [77]. Graphene-based applications are also under investigation in the fields of sensor development, biology and medical sciences $[19,78]$. To date, however, most of these applications are not within immediate reach due to still existing challenges, mainly connected to the complexity of large-scale manufacturing of high-quality graphene sheets [19].

In contrast, the first applications using graphene as a compound material, in particular within a polymer matrix, are already available today [21, 79-82]. So far, applications 
include graphene-based touch-screens [83-85], coatings for thermal and electromagnetic shielding and conductive $\mathrm{ink}^{2}$.

Yet, before graphene composite materials are discussed, a brief introduction to graphene is presented here. In view of the analysis performed in this work, this introduction will mainly focus on the structural properties of graphene. A broader and more general review of graphene can be found in Ref. [19, 72].

\subsubsection{Structural properties of graphene}

Graphene consists of a single layer of carbon atoms arranged in a hexagonal lattice with a two-atomic base (Fig. 2.6) [86]. The corresponding reciprocal lattice therefore also exhibits a six-fold symmetry with reciprocal lattice vectors as depicted in Fig. 2.5A.

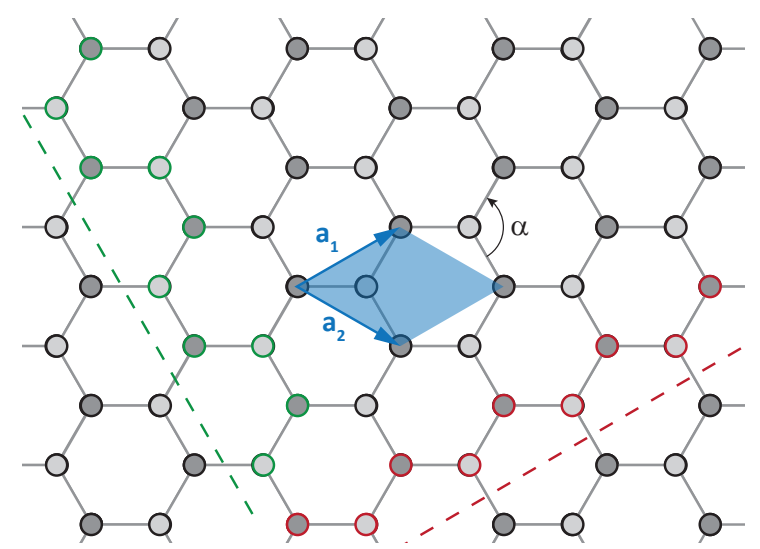

Figure 2.6: Hexagonal lattice of graphene $\left(\alpha=120^{\circ}\right)$. Possible representation of the unit cell indicated in blue with unit cell vectors $\mathbf{a}_{1}$ and $\mathbf{a}_{2}$. Atoms of the two sub-lattices are shaded with dark and light gray. Possible grain boundaries for polycrystalline graphene: Cut along [10] direction leads to zigzag (red), cut along [-12] direction leads to armchair configuration (green). Reciprocal lattice as in Fig. 2.5A.

The four binding electrons of each carbon atom hybridize into three covalent $\mathrm{sp}^{2}$ bonds and a single, delocalized $\pi$ bond [87]. The carbon-carbon bond length is $1.42 \AA$, leading to a unit cell vector length of $\left|\mathbf{a}_{1}\right|=\left|\mathbf{a}_{2}\right|=\sqrt{3} \cdot 1.42 \AA=2.46 \AA$ [88]. The covalent bonding energy within the lattice plane is large $\left(E_{\text {inplane }}=4.3 \mathrm{eV}\right)$ compared to the van der Waals bonding energy between adjacent, stacked sheets in the case of graphite $\left(E_{\text {normal }}=0.07 \mathrm{eV}\right)[87]$. The successful exfoliation of graphite to isolated single sheets of graphene can be attributed to this discrepancy [71].

\footnotetext{
${ }^{2}$ List of vendors of a selection of graphene-based applications can be found here: http://www.understandingnano.com/graphene-companies.html.
} 
Due to the honeycomb structure of the lattice, different types of grain boundaries for polycrystalline graphene are possible. Grain boundaries can in principle strongly influence the mechanical and electronic properties of the material by the formation of defects [89-91]. In the case of graphene, however, it was found that for large tilting angles the incorporation of defects into the crystal lattice does not necessarily lead to a drastic decrease in bond stability. In particular, tilting angles of $21.7^{\circ}$ (zigzag configuration, red dashed line and atoms in Fig. 2.6) and $28.7^{\circ}$ (armchair configuration, green dashed line and atoms in Fig. 2.6) lead to less initial strain on the carbon bonds compared to smaller angles in the same configuration, and are therefore very stable [91].

\subsubsection{Polymers in graphene technology}

As previously mentioned, potential graphene applications do not only arise from the use of pure graphene, but also from the combination of graphene with different other materials. For the latter case, two major current research areas can be identified, namely graphene-based heterostructures and composite materials.

Heterostructures describe a group of thin-film type materials, which are constructed by consecutive stacking of individual two-dimensional crystalline sheets on top of each other [18]. Whereas strong covalent bonds act within these sheets, the resulting heterostructures are held together by relatively weak van der Waals forces [92, 93].

The big appeal of such structures stems from the idea to atomically tailor material properties. With the large amount of readily available $2 \mathrm{D}$ crystals $[18,19]$, this provides for a seemingly endless number of possible combinations. Specific efforts are, for example, dedicated to find high temperature superconductors [18] or substituents for silicon-based electronics [94].

A conceptually similar approach to heterostructures is the manufacturing of composite materials. Here, too, the modification of material properties is in the foreground, which is commonly accomplished by immersion of graphene sheets or flakes (so called nano-platelets) in a polymer matrix [21, 79, 95-97]. From the technological point of view, graphene-based composite materials therefore offer one great advantage compared to heterostructures: Since they do not necessitate layer-based, large-area, high-quality, 
single-crystalline graphene, but mostly rely on nano-platelets of few to few tens of micrometers in diameter, the production process is greatly facilitated. At very low volumetric fractions of graphene, significant increases in tensile strength, Young's modulus, as well as thermal and electric conductivity have been reported [98]. The resulting features make these new materials interesting for a wide range of applications, as, for example, conductive plastics and ink as used in electromagnetic interference shielding [77], or implementation into energy conversion [99], energy storage [100] and non-volatile memory devices [101].

Polymers, including poly(methyl methacrylate) (PMMA) and polycarbonate (PC), are commonly used to stabilize the two-dimensional crystal sheets upon transfer from the substrate used in the preparation process [102] (Sec. 6.1.1). After the transfer process, the thin polymer film is removed by organic solvents such as acetone and isopropanol [103]. However, the strong physisorption of the polymer in contact with the graphene results in a very resistant ultrathin residual polymer layer [104, 105]. Several methods have been employed to remove this residual layer, e.g. changing the polymer [106], annealing at high temperatures [105], as well as polymer-free, so-called direct transfer [107]. However, to date, none of these approaches has resulted in large area, high quality graphene, stimulating additional interest in the investigation of the intimate connection between these two unlike materials $[21,108,109]$.

\subsubsection{Structural properties of PMMA}

PMMA is a lightweight plastic, which is most commonly known as acrylic glass (trade name: Plexiglas). Low cost and easy handling as well as the lack of potentially harmful bisphenol-A, as found in PC, allow PMMA to be used in many applications in medical technologies or as a resist in semiconductor electron beam lithography.

Structurally, this polymer consists of long chains of methyl-methacrylate (MMA) repeat units as shown in Fig. 2.7A and B. The molar mass of MMA is $100.12 \mathrm{~g} / \mathrm{mol}$, while the total polymer's molar mass strongly depends on the overall chain length, and is usually in the range of $5 \cdot 10^{4}-10^{6} \mathrm{~g} / \mathrm{mol}$.

PMMA molecules can display different tacticity, which describes the relative orientation of the side groups within the polymer. Possible configurations are isotactic (Fig. 2.7C), 

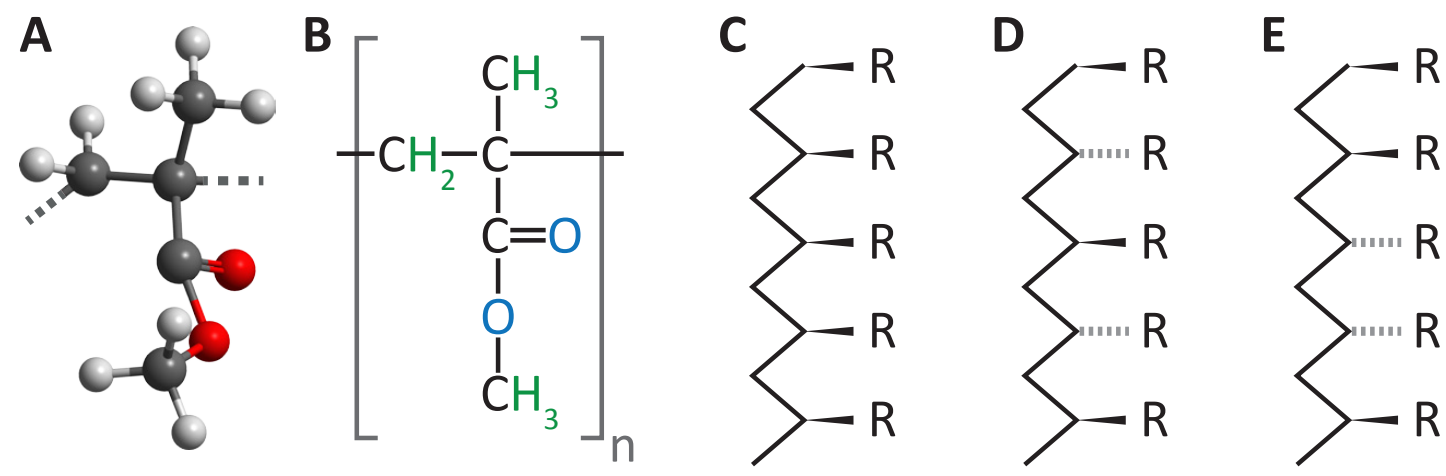

Figure 2.7: Structure and tacticity of PMMA. A,B: 3D model and structural formula of repeating monomer unit MMA. C-E: Sketch of isotactic, syndiotactic and atactic configurations, respectively. $R$ represents the functional (side) group of the monomer.

syndiotactic (Fig. 2.7D) and atactic (Fig. 2.7E). Tacticity strongly influences the physical properties of a polymer, including the degree to which it exhibits crystalline order.

STM as well as AFM studies have shown that thin PMMA Langmuir-Blodgett films on mica and graphite display long-range crystalline order independent of tacticity, in contrast to PMMA behavior in bulk $[110,111]$. This behavior is in principle well known for other types of polymers, too, when subjected to a strong potential template [112, 113]. In the case of graphite and graphene, the adsorption of polymer chains to the surface is facilitated via van der Waals forces, similar to the bonding between consecutive graphene planes [114].

Atactic and syndiotactic PMMA molecules both arrange in a folded-chain configuration with little to no backbone crossovers [110]. The chain-to-chain distances reported in the literature are 5.0(1.0) $\AA$ and 4.8(1.9) $\AA$, respectively, with a repeating monomer unit length of about $2.5 \AA$ in direction of the polymer backbone. In the case of isotactic PMMA, linear as well as helical arrangements are found with a somewhat smaller interchain distance of the latter of $3.7(0.8) \AA[110,115,116]$.

Because of the sensitivity of PMMA to electron irradiation, the formation of crystalline folded-chain conformations is challenging to observe in a regular transmission electron microscope (TEM), because of the relatively high electron current density of such systems. For ULEED, a quantitative analysis of the degradation behavior can be found in App. B.4. 



\section{Chapter 3}

\section{Aspects of ultrafast LEED}

In this chapter, the potential of ultrafast LEED for investigations with atomic-scale resolution is discussed. To motivate the development of an ULEED setup, the field of ultrafast science is briefly introduced with focus on the time-resolved study of quasi-twodimensional systems (Sec. 3.1), while particular challenges in such a setup are assessed in the subsequent section (Sec. 3.1.1). Next, an introduction is given into common techniques employed in ultrafast imaging and diffraction. In particular, the pump-probe scheme is described (Sec. 3.2), followed by a brief presentation of different electron sources evaluated with respect to their capability to be implemented into a time-resolved LEED experiment (Sec. 3.3). Lastly, the characteristic quantities of a pulsed electron source based on a nonlinearly driven nanometric photocathode are determined (Sec. 3.4) and discussed in view of an application in ULEED (Sec. 3.5).

\subsection{Motivation}

The field of ultrafast science is intimately connected to the development of mode-locked laser sources in the 1960s [117], which ultimately allowed for the observation of extremely short-lived phenomena $[118,119]$ on time scales below those accessible via electronic means $[47,120]$.

In 1984, Williamson et al. investigated the fluence-dependent melting of a thin aluminum sample by diffraction upon illumination from a strong laser pulse, using electrons with a kinetic energy of $25 \mathrm{keV}$ [121]. And even though for higher fluences, the achieved 
pulse duration of 20 ps only allowed for an observation of the loss of order in the sample at the time, the applied methodology to study ultrafast phenomena remained mostly unchanged until today. Specifically, laser-driven photocathodes in combination with a pump-probe scheme are still the basis for the majority of modern ultrafast electron diffraction and imaging experiments [33, 122, 123].

Since then, a multitude of techniques with ultrahigh spatio-temporal resolution have emerged, including time-resolved photoelectron spectroscopy [124, 125], ultrafast x-ray spectroscopy $[126,127]$ and crystallography $[40,41,128]$, high-energy electron diffraction [33, 34, 129] and microscopy [36, 37], as well as, relatively recently, terahertz STM (THz-STM) [130]. The type of probe pulse in combination with the geometry of the experimental setup determine, which quantities of a system can be accessed.

Generally, compared to the large number of techniques with atomic resolution available for the observation of ultrafast phenomena in bulk media, accessing dynamics at surfaces with similar resolution has proven to be challenging. Time and angle-resolved photoemission spectroscopy (trARPES) [125] and THz-STM can be, for example, employed to map the electronic structure with atomic resolution and high surface sensitivity. For the investigation of the atomic structure at surfaces, mainly two pathways are currently followed: On the one hand, extremely thin films are probed by ultrafast TEM (UTEM) [131] and ultrafast electron diffraction (UED) [132] in transmission. To obtain a strong surface signal, a grazing incidence geometry is alternatively applied as in time-resolved XRD [133] and RHEED [44] studies.

Existing time-resolved surface studies have demonstrated the richness of ultrafast phenomena in quasi-two-dimensional systems excited to states far from equilibrium, including phonon confinement effects in ultrathin films [134], the relaxations of surface reconstructions and complex superstructures in monolayer adsorbate systems [44, 135], surface pre-melting [136], and the formation of warm dense matter as a result of a strong coupling between electronic and lattice systems [137].

But despite recent accomplishments, e.g., in further increasing the temporal resolution of the experimental techniques by pulse compression schemes [138-140] and tilted wavefront setups $[35,45]$, the inherent requirements to achieve high surface sensitivity make quantitative analysis difficult. Particular challenges are the strong influence of the surface morphology, large probing areas, and the mapping of only a restricted 
angular fraction of reciprocal space in grazing incidence-based methods, as well as the preparation of ultrathin samples to avoid large background contributions in ultrafast transmission experiments.

LEED equipped with ultrafast temporal resolution, on the other hand, would naturally offer extremely high surface sensitivity in combination with a well-developed experimental and theoretical framework to access the atomic structural evolution at surfaces.

\subsubsection{Particular challenges of ultrafast LEED}

An implementation of ultrahigh temporal resolution into a LEED system using a laserpump / electron-probe scheme as described below is mainly limited by the capability to deliver short electron pulses at low energies [46, 47, 141, 142]. In particular, electrons with kinetic energies typically employed in LEED are highly susceptible to spatiotemporal broadening from velocity dispersion and space charge effects.

A temporal expansion by Coulomb repulsion within the bunch can, for example, be avoided by operating in the one-to-few electrons per pulse regime, while integrating over a large number of electron pulses. Alternatively, electron pulses can be compressed, e.g., within a radio-frequency cavity [138], to improve the temporal resolution. Yet, considering the proof-of-concept character of the ultrafast LEED developed in this work as well as the confined dimension of a LEED setup, a complex pulse compression scheme as employed in high-energy electron diffraction experiments seems to be too involved at this point in time. Hence, the ultrafast LEED setup in this work is based on the stroboscopic approach introduced in the next section, avoiding space-charge effects by employing only a relatively small electron number per pulse.

In terms of dispersive broadening caused by different initial kinetic energies of the emitted electrons, several solutions have been suggested. However, many of the proposed ideas make compromises in either low signal intensity [142] or grazing incidence geometry [143], which ultimately complicates the quantitative analysis, as well as limits the achievable pulse duration.

As an alternative approach, electron sources based on the nonlinear emission from nanometric photocathodes have been proposed [48, 49, 51-54]. In the following, after a brief 
description of the pump-probe scheme used in ultrafast imaging and diffraction, an introduction to the operation principle of tip-based photocathodes is given as well as a more detailed analysis in terms of their applicability with respect to an ultrafast LEED experiment.

\subsection{Ultrahigh temporal resolution via pump-probe}

Ultrafast temporal resolution is commonly achieved by so-called pump-probe schemes, as illustrated in Fig. 3.1. The sample is pumped by a short laser pulse (red) and subsequently probed by an electron pulse (green) after a given temporal delay. In particular, the electron pulse is generated within a fast photocathode, which is driven by part of the same laser pulse (purple) employed also for the sample excitation.

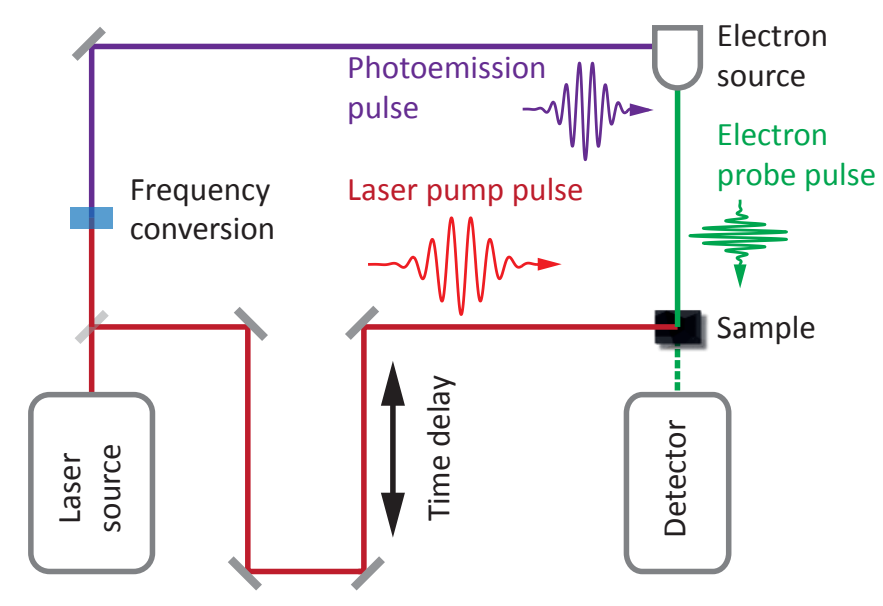

FigurE 3.1: Sketch of a laser-pump / electron-probe scheme. The relative arrival time is controlled by changing the optical path length of the laser pump pulse (red).

The challenge of realizing exact timing of the relative arrival of (laser) pump and (electron) probe pulses is solved by introducing a variable additional optical path length in either the pump or the photoemission arm of the laser. In particular, a routinely controllable additional optical path length of $1 \mu \mathrm{m}$ translates into a temporal shift of $3.3 \mathrm{fs}$, thus allowing for extremely high temporal accuracy.

Depending on the experimental settings, this scheme can be operated to obtain information in real or in reciprocal space. Diffraction techniques provide direct insight into the structure of a system by measuring the specimen's atomic spacings, whereas real space images can be employed to identify phase and grain boundaries, impurities, dislocations or defects in the sample $[43,144]$. The requirements in terms of electron sources for 
both cases are similar though, and depend strongly on the investigated sample as well as the employed experimental geometry. For imaging as well as for diffraction, two main modes of operation can be identified.

The single-shot approach is mainly used for the investigation of irreversible dynamics, allowing for an observation of the dynamics as they occur [139, 145]. In order to extract a significant amount of information from the sample with a single shot, probing pulses with about $10^{7}$ electrons per pulse are required to obtain an image. Such dense electron clouds are highly susceptible to internal Coulomb repulsion, which eventually limits the currently achievable temporal resolution to few tens of nanoseconds in TEMs [146] and some hundreds of femtoseconds for UED [147]. Several techniques, such as radiofrequency compression as well as relativistic electron energies, are commonly employed to reduce temporal pulse broadening [148-151].

In an alternative operation mode, the so-called stroboscopic or single-electron approach, individual images are formed by integration over a large number of probing pulses including only a small number of electrons in each one [152]. Hence, space charge effects can be mainly avoided, allowing for a temporal resolution down to few hundreds of femtoseconds in imaging as well as diffraction. Compared to the single-shot approach, comparable or even higher temporal resolution can be achieved with considerably less involved experimental setups [146]. However, a necessary requirement to the investigated dynamical process is its reversibility on a time scale determined by the repetition rate of the driving laser system, which is somewhat limiting the accessible processes and sample systems. On the one hand, generally reversible systems will most likely relax to their initial state in between consecutive pump pulses, given the ultrafast nature of the investigated processes and commonly employed laser repetition rates in the $\mathrm{kHz}$ to $\mathrm{MHz}$ regime. On the other hand, the sample must be able to withstand not only about $10^{6}$ to $10^{8}$ such pulses, but also an equal number of photo excitations, potentially leading to a degradation of the sample, e.g. by accumulative heating [139]. These effects may be particularly severe when studying organic molecules, as for example proteins or polymers. Then again, the life span of very sensitive samples might strongly benefit from the small duty cycle of the experiments, allowing for the dissipation of heat and charge in between consecutive excitations (The sample degradation for the polymer investigated in Chap. 6 is described in App. B.4.). 
In order to further increase the experimental capabilities of both approaches, much effort is currently devoted into the development and modification of novel electron sources $[43,131,153]$.

\subsection{From continuous to pulsed electron sources}

\subsubsection{Thermionic electron sources}

Electron sources as used in experimental setups such as LEED and TEM are usually operated by heating a metal filament connected to a sharp metal tip. By raising the temperature of the material, the Fermi-Dirac distribution of the electron gas is broadened, eventually allowing electrons from the high-energy tail to overcome the potential barrier and subsequently be emitted into the vacuum [154]. These so-called thermionic electron sources rely strongly on the thermal stability with respect to the work function of the employed materials, and are therefore commonly made from tungsten (W). Alternatively, Lanthanum hexaboride $\left(\mathrm{LaB}_{6}\right)$ electron sources are frequently used in TEMs, because of the material's very low work function (around $2.7 \mathrm{eV}$ ) in combination with a high melting temperature $\left(2210^{\circ} \mathrm{C}\right)[62]$.

\subsubsection{Cold and Schottky field emitters}

However, the operation at temperatures necessary for thermal emission strongly reduces the lifetime of thermionic electron sources to at most a few hundred hours [62]. To lessen the strain on the material, different approaches are pursued: In order to decrease the necessary thermal energy of the electrons for the emission process, the effective work function $\Phi_{\text {eff }}$ can be decreased by the application of an electric field $F$ to the emitter $[155,156]$

$$
\Phi_{\text {eff }}=\Phi-\sqrt{\frac{e^{3} F}{4 \pi \epsilon_{0}}},
$$

with $\Phi$ the material's work function, $e$ the electron charge and $\epsilon_{0}$ the vacuum permittivity. Specifically, an applied electric potential reduces the effective image potential of the electron cloud at the surface of the metal and hence the work function. 
The idea of so-called cold field emission guns is to employ the lightning rod effect of sharp metal tips to locally enhance the electric field, resulting in strongly localized electron emission at the tip apex [48, 157-159]. Particularly, for a given potential $U$ and a tip radius of curvature $r$, the enhanced field strength is given by $F=U /(k r)$, with $k \approx 5-6$ a geometrical factor depending on the shape of the tip and its distance to the anode [160, 161]. The typical radius of curvature of employed field emission tips is of the order of about $100 \mathrm{~nm}$, leading to significant field enhancement. In addition to the operation at much lower temperatures, which significantly increases the source's lifetime, field emission guns exhibit a narrower electron energy spectrum [62]. Furthermore, the smaller emission area leads to more favorable beam properties, in particular an increased brightness of the source (see Sec. 3.4.3) as well as a higher transverse coherence (see Sec. 3.4.1).

For so-called Schottky field emitters, tungsten tips are additionally coated with materials such as zirconium dioxide $\left(\mathrm{ZrO}_{2}\right)$ to locally lower the work function $[62,162]$. In contrast to cold field emitters, which are normally operated at room temperature, Schottky field emitters are operated at around $1800 \mathrm{~K}$. They usually feature higher total emission currents, but lower emission current densities due to their larger cathode radius. Therefore, even though Schottky field emitters have the advantages of not requiring frequent cleaning and as strict vacuum conditions, cold field emitters offer higher brightness and smaller emittance.

\subsubsection{Pulsed electron sources}

To achieve precise temporal control over the emission process, pulsed electron sources are commonly driven by intense laser pulses [150, 163-165]. Most state of the art femtosecond photocathodes are based on the classical photoelectric effect, i.e., for moderate light intensities, electron emission occurs upon absorption of a photon with an energy $\hbar \omega$ above the work function $\Phi$ of the material (Fig. 3.2A). This mode of operation is usually realized in pulsed electron sources by the illumination of a thin metal film and a subsequent acceleration of the emitted electrons within a strong electric potential $[33,143,166]$. However, this approach is challenging to implement in LEED-type experiments, since pulses of low-energy electrons are very susceptible to dispersive broadening even at narrow electron energy distributions and the absence of space charge effects. 
Instead, as will be discussed later (Sec. 5.3.2), pulsed electron emission within a field emitter geometry can be used to strongly minimize these effects.
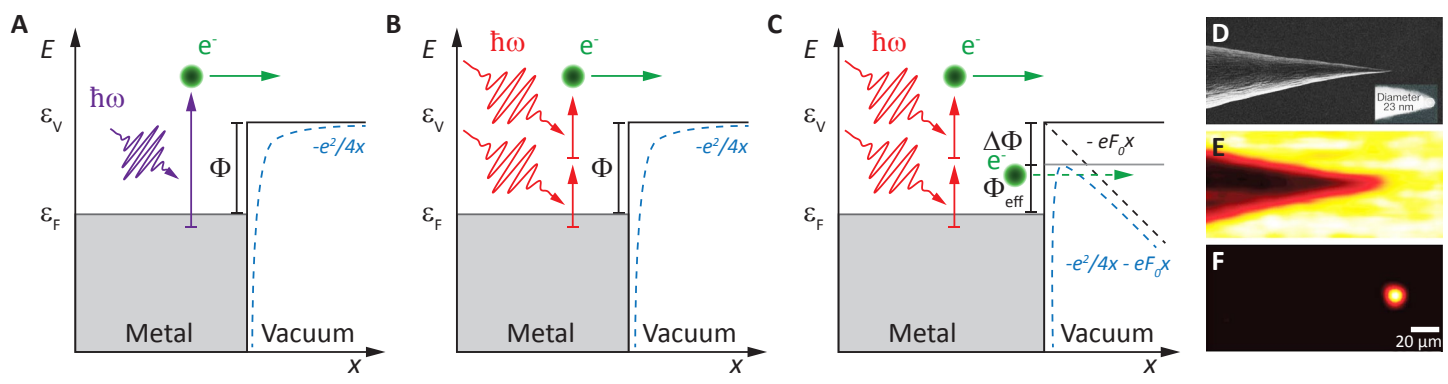

Figure 3.2: Comparison of linear and nonlinear photoemission. A: Sketch of the classic photoelectric effect. Photon with energy above metal's work function $(\hbar \omega>\Phi)$ raises electron energy from Fermi-level $\left(\epsilon_{F}\right)$ to above vacuum level $\left(\epsilon_{V}\right)$; electron is emitted. Potential decay indicated by blue dashed line. B: Sketch of multiphoton photoelectric effect. Single photon energy below work function of the metal $(\hbar \omega<\Phi)$, vacuum potential barrier energetically overcome by absorption of multiple photons. C: Tunnel assisted multiphoton photoelectric effect aided by static field $F_{0}$ to lower the work function (Schottky effect) to $\Phi_{\text {eff }}=\Phi-\Delta \Phi$ (dashed green arrow). D-F: SEM image of sharp gold tip (D), shadow image (E) and strongly localized electron signal

(F) of same tip, when scanned through the laser focus. [167]

The translation of the field emitter working principle is not directly transferable to pulsed operation. In particular, an illumination of a sharp needle-type photocathode with photons of energy $\hbar \omega>\Phi$ does not lead to strongly localized emission as described above. In this case, the size of the emission area is given by the diameter of the laser pulse on the photocathode and therefore effectively by the used wavelength.

In order to accomplish selective electron emission, nanometric sources can instead be illuminated by light pulses of photon energies below the material's work function, eventually resulting in nonlinear (multiphoton) photoemission for high enough light intensities (Fig. 3.2B). In this case, several photons with $\hbar \omega<\Phi$ are absorbed to deliver the necessary energy to overcome the potential barrier for electron emission. However, the absorption of multiple photons by the same electron requires very high optical field amplitudes. High fields can be reached by intense, short laser pulses in combination with the above mentioned field enhancement effects at regions of small radius of curvature $[50,168,169]$. This results in a highly selective emission process for a polarization of the driving light field along the tip axis, ultimately leading to an extreme localization of the electron emission site (Fig. 3.2D-F)[157, 170, 171]. Furthermore, electron emission can be supported by the additional application of a static potential to the tip to reduce the effective work function (Fig. 3.2C) [49, 50, 172]. 
This description holds, as long as the light fields are still significantly smaller than the binding fields of the material. The relationship between the strengths of light and binding energy is usually quantified by the Keldysh parameter $\gamma$, defined as [173]

$$
\begin{aligned}
\gamma & =\sqrt{\frac{\Phi}{2 U_{p}}} \text { with } \\
U_{p} & =\frac{e^{2} F^{2}}{2 \epsilon_{0} c m_{e} \omega^{2}},
\end{aligned}
$$

where the latter is the ponderomotive potential of the light field $F$ oscillating at an frequency of $\omega$. Physically, $U_{p}$ describes the cycle-averaged quiver energy of an electron (charge $e$, mass $m_{e}$ ) in an oscillating electromagnetic field $F$. For $\gamma \gg 1$, the light field can be seen as a small perturbation of the binding potential (multiphoton regime), while the latter is dominated by the former in case of $\gamma \ll 1$ (optical field emission or strongfield regime). A more detailed treatment of electron emission within the strong-field regime can be found in Refs. $[174,175]$ and references therein. The potential operation of an electron gun in the optical-field emission regime is briefly discussed at the end of Chap. 5 (Sec. 5.4.3).

\subsection{Beam properties of tip-based electron sources}

In the following, an estimate of the characteristic quantities of the proposed tip-based electron source will be given, namely coherence (Sec. 3.4.1), emittance (Sec. 3.4.2), brightness (Sec. 3.4.3) and temporal resolution (Sec. 3.4.4). Furthermore, a performance comparison with state of the art pulsed electron sources is provided (Fig. 3.3).

A

$(-)$

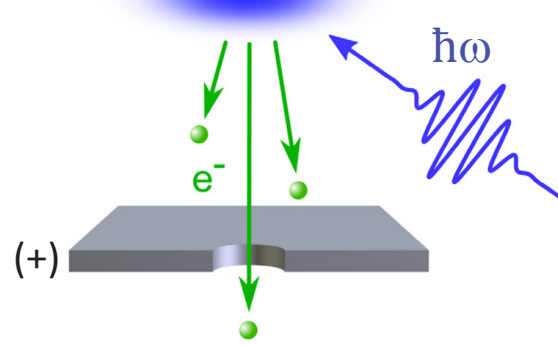

B

$(-)$

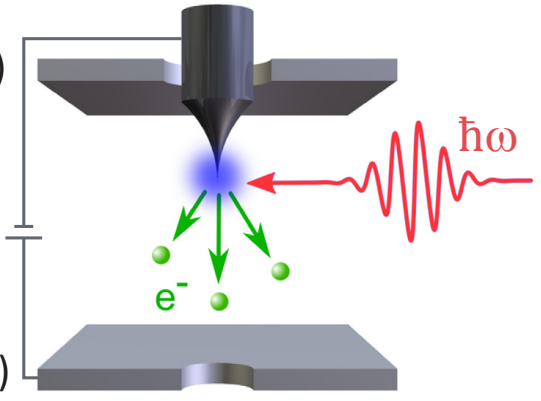

FiguRE 3.3: Sketches for planar (A) and tip-based (B) electron gun geometry. [176] 


\subsubsection{Coherence}

In an electron diffraction experiment, the pattern on the detector results from the superposition of electron wave packets, which have been scattered by the crystal lattice of the sample. The coherence describes the phase correlation within an ensemble of electron wave packets and hence their ability to interfere ${ }^{1}$.

Generally speaking, there are two types of coherence, namely a longitudinal (temporal) coherence, and a transverse (spatial) coherence (Fig. 3.4)[177]. The former is a measure of correlation between the phase of the wave in time and in propagation direction. A phase shift can be introduced, for example, by different initial kinetic energies of the electrons. In a typical diffraction experiment, the longitudinal coherence length is much larger than the spacing between atoms in the direction of propagation and therefore poses no limitation for the observation of diffraction spots, especially in quasitwo-dimensional systems [140]. A small longitudinal coherence length will mainly result in an incoherent superposition of diffraction spots corresponding to different energies and hence contributes only to the overall intensity of the diffraction pattern [178].

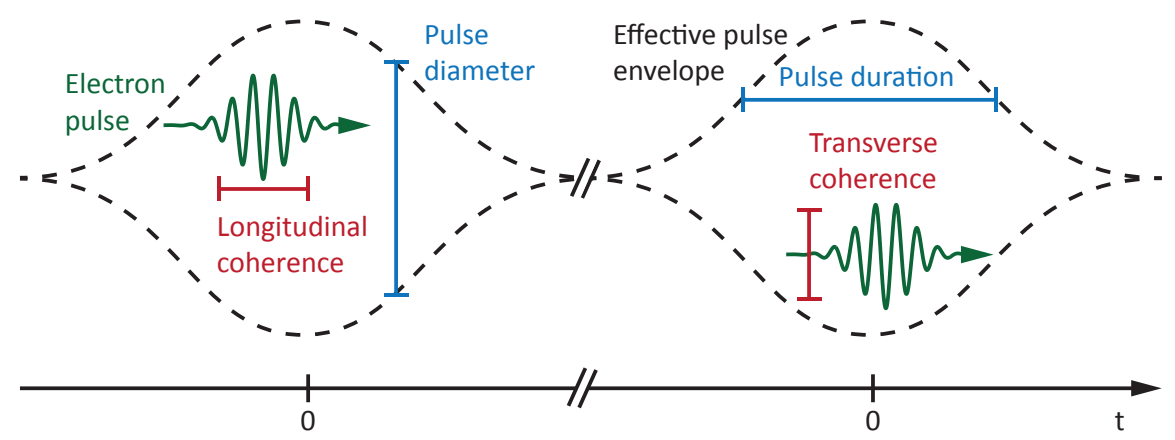

Figure 3.4: Transverse (spatial) and longitudinal (temporal) coherence of electron pulses. Due to spatial and temporal jittering, pulse diameter and duration are determined by the effective pulse envelope. Relative laser pulse timing indicated at $t=0$. Image adapted from Ref. [140].

In contrast, the transverse coherence is a measure for the ability of scatterers to interfere when separated normal to their direction of propagation. It ultimately limits the maximum spatial resolution of a diffraction experiment, i.e., the achievable sharpness of the recorded Bragg peaks [177]. Ideally, the sharpness is only determined by the spatial extension of the sample region within which scattered electrons from the source can still constructively interfere. Therefore, the coherently probed sample region should be as

\footnotetext{
${ }^{1}$ In a more general definition as used in Ref. [177], the coherence is a measure for the correlation between quantities of an optical field.
} 
big as possible, which can be a limiting factor especially in the case of very large unit cells, e.g., from long organic molecules [139].

A

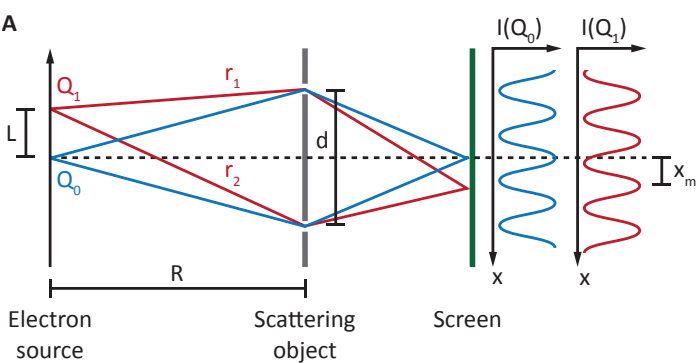

B

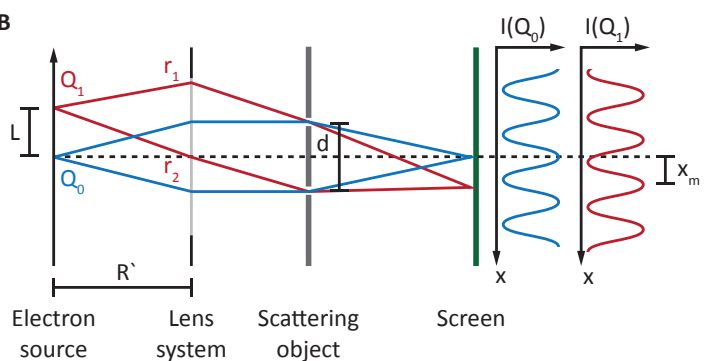

FIGURE 3.5: Sketches of interference experiments. A: Classical Young's interference setup displaying two optical paths from points $Q_{1}$ (red) and $Q_{0}$ (blue) of the source. B: Setup with additional lens system at position $R^{\prime}$. Beams between $R^{\prime}$ and the screen are assumed to propagate parallel. [179]

Figure 3.5A illustrates the setup of a classical interference experiment with an extended, incoherent (electron) source of size $L$. here, incoherent means that there is no fixed phase relation along the source's spatial extent. Instead, the source can be seen as an array of independent point sources. For simplicity, a double slit of dimension $d$ is taken to be the scattering object at distance $R$ from the source. The path of waves emitted from a point $Q_{0}$ on the optical axis and the resulting interference pattern on the screen are indicated as blue lines. Since no path length difference occurs, the intensity maximum is found on the optical axis (see $\left.I\left(Q_{0}\right)\right)$.

The propagation paths $r_{1}$ and $r_{2}$ of electrons from the outmost point $Q_{1}$ of the source to the scattering object, and subsequently to the screen, are marked as red lines. In contrast to emission from the optical axis, the path length difference for electron emitted from $Q_{1}$ is non-zero and instead given by [177]

$$
\left|r_{2}-r_{1}\right| \approx \frac{d L}{R}
$$

For Eq. 3.4, it was assumed that the extent of the source $\left(\overline{Q_{1} Q_{2}}\right)$ is small compared to the distance $R$ to the scattering object. As a result from the off-axis emission, the intensity pattern $I\left(Q_{1}\right)$ is shifted with respect to the one generated from $Q_{0}$. Specifically, destructive interference is obtained when the intensity maximum of $Q_{0}$ coincides with the intensity minimum of $Q_{1}$ or vice versa. In this case, the path difference would be exactly half the electron wavelength. 
This consideration allows for the determination of the maximum extent of the sample area within which scattered electrons can interfere constructively:

$$
d_{c}=\frac{\lambda R}{2 L}
$$

Usually, electrons are not propagating freely to the sample, but are subject to collimation, e.g., by an electrostatic lens system at a distance $R^{\prime}<R$. For the purpose of generality, we assume that the propagation paths of electrons from the same point of the source are parallel after the source (Fig. 3.5B), hence $R=R^{\prime}$ in Eq. 3.5. As can be seen from Eq. 3.5, $d_{c}$ is inversely proportional to the spatial extension of the source. With the emission area of a tip source of the order of its radius of curvature (about $10 \mathrm{~nm}$ ), a comparison to a planar photocathode, where the source size is determined by the size of the laser focus (about $10 \mu \mathrm{m}$ ), yields an increase of about three orders of magnitude in the transverse coherence. This would translate in a six orders of magnitude larger area of unit cells within the coherently illuminated sample surface, potentially leading to significantly sharper Bragg peaks [180]. However, the present discussion is not taking into account aberrations caused by the lens system or a limited resolution of the detector assembly.

For parameters typically employed in LEED experiments $\left(\lambda=1 \AA, R^{\prime}=1 \mathrm{~mm}\right)$ a maximum transverse coherence length of few tens of micrometers can be theoretically achieved within the tip geometry. In comparison, standard LEED optics only exhibit transverse coherence lengths of $10-20 \mathrm{~nm}$ at most [61]. Consequently, the enlarged coherence would potentially allow not only for sharper diffraction patterns in general, but also for the investigation in deviations of long range order on the micrometer scale not observable with current electron sources.

\subsubsection{Emittance}

As mentioned in the previous section, electron beams are not monochromatic, but have a finite kinetic energy spectrum. The reasons for this energy spread are manifold and include emission from different energy levels within the metal, modifications in the effective work function of the particular emission site, and in case of photoemission also non-monochromatic photons from the driving source. The emittance provides a figure of merit for the beam quality by relating it to the phase space volume or the beam's width 
and divergence. Since the exact definition of the emittance is not congruent throughout the literature, we will follow the convention of M. Reiser [181] and Dowell et al. [156] for this purpose.

The root-mean-square (RMS) emittance as a measure of the pulse's spatial extension and divergence and is defined as

$$
\epsilon_{\mathrm{RMS}}=\sqrt{\left\langle r^{2}\right\rangle\left\langle r^{\prime 2}\right\rangle-\left\langle r r^{\prime}\right\rangle^{2}}
$$

with $\langle\ldots\rangle$ denoting the average value of the quantity in brackets, $r$ the radial position in cylindrical coordinates, and $r^{\prime}=d r / d z$ the slope along the direction of propagation. The cross term $\left\langle r r^{\prime}\right\rangle^{2}$ encompasses the correlation between the location of emission and the transverse momentum and is zero at the waist of an ideal uniform beam. As a result, the RMS emittance is a measure for the minimal achievable cross section $\sigma_{r}=\sqrt{\left\langle r^{2}\right\rangle}$ of the beam, which is given by

$$
\sigma_{r}=\epsilon_{\mathrm{RMS}} / \sigma_{r^{\prime}}
$$

with $\sigma_{r^{\prime}}=\sqrt{\left\langle r^{\prime 2}\right\rangle}$ the RMS divergence of the electron beam.

For a flat photocathode, it is assumed that the emission site and the transverse momentum of the emitted electron are generally not correlated, hence $\left\langle r r^{\prime}\right\rangle^{2}=0$. This consideration leads to the following expression for the RMS emittance $\epsilon_{n}$, normalized by the relativistic factor $\beta / \sqrt{1-\beta^{2}}$ (with $\beta=v_{e} / c$ and $v_{e}$ and $c$ the electron and light velocity, respectively) to compensate for different electron pulse operation energies:

$$
\epsilon_{n} \approx \sigma_{r} \sqrt{\frac{\hbar \omega-\Phi_{\mathrm{eff}}}{3 m_{e} c^{2}}} .
$$

Here, $\hbar \omega-\Phi_{\text {eff }}$ is the excess energy from the photoemission process with $\Phi_{\text {eff }}$ as given in Eq. 3.1 and $m_{e}$ the electron's mass. For electrons photoemitted from a tungsten surface within a well-adjusted two-photon process (excess energy: $0.1 \mathrm{eV}$ ) from an emission site of $20 \mu \mathrm{m}$ diameter, Eq. 3.8 would result in a normalized RMS emittance of about 2.5 $\mu \mathrm{m}$ mrad, which is in the typical range of values for such a emitter $[166,182]$.

In case of a needle emitter, the electron momentum is correlated to the emission site on the apex, hence $\left\langle r r^{\prime}\right\rangle^{2} \neq 0$. Consequently, the effective source size in a tip geometry is smaller than the radius of curvature of the emission site. A pulsed electron source based on a nanometric needle-type emitter with a drastically decreased emission area is 
therefore beneficial in terms of the achievable emittance. Specifically, S. Strauch [182] performed a detailed FEM simulation-based analysis and comparison of the emittance and brightness of a pulsed tip-based electron source with respect to flat cathode emitters in the framework of the development of an ultrafast TEM setup. According to the numerical findings in her work, the achievable emittance in such a geometry is significantly smaller than in the case of the flat emitter type at a comparable brightness.

\subsubsection{Brightness}

Even though the emittance is a valuable figure of merit for the beam quality, it can theoretically be arbitrarily reduced by the placement of collimating apertures lessening the transversal spread. This would of course severely diminish the overall current available for imaging and diffraction. Consequently, and in perspective of the instrument's capability to resolve atomically small features within a reasonable time, the current has to be taken into account when characterizing the electron beam. The parameter used to describe this requirement is the brightness $B$, which is the current density $J=d I / d A$ per unit solid angle $d \Omega$,

$$
B=\frac{d I}{d A d \Omega}
$$

The brightness averaged over the whole trace space volume can be expressed in terms of the RMS emittance $\epsilon_{n}$ via

$$
\bar{B}=\frac{I}{8 \pi^{2} \epsilon_{n}^{2}} .
$$

As in the case of the emittance, the brightness can be arbitrarily increased when minimizing the solid angle of emission by the application of high acceleration voltages. Therefore, the brightness is usually normalized by the relativistic parameter $(\beta \gamma)^{2}$ similarly to the emittance or in terms of the accelerating voltage ${ }^{2} U$. The so called reduced brightness then reads

$$
\bar{B}_{r}=\frac{\bar{B}}{U}=\frac{I}{8 U \pi^{2} \epsilon_{n}^{2}} .
$$

Typical values for static thermionic electron guns are of the order of $10^{6} \mathrm{~A} / \mathrm{Vm}^{2} \mathrm{sr}$, those for field emission guns even of $10^{12} \mathrm{~A} / \mathrm{Vm}^{2} \mathrm{sr}[62,162,182,183]$. In comparison to these static sources, pulsed electron guns have the disadvantage of a very small duty cycle,

\footnotetext{
${ }^{2}(\beta \gamma)^{2} \approx 2 \cdot 10^{-6} U$
} 
thus reducing the average brightness by orders of magnitude, making it difficult to judge their applicability.

Therefore, we compare the reduced brightness to be expected for an ultrafast tip-based emitter with those of flat state of the art femto- and picosecond photocathodes, which have already been successfully employed in time-resolved imaging and diffraction experiments. Values for the latter range between $10^{-4}$ and $10^{3} \mathrm{~A} / \mathrm{Vm}^{2} \mathrm{sr}[147,166,184]$.

For the computation of the reduced brightness in a needle-type geometry, we assume an emission area similar to the apex surface of a typical tip of $A=\pi(10 \mathrm{~nm})^{2}$, while the solid angle of emission without a lens system is about $\Omega=0.3 \mathrm{sr}$ [50]. With an acceleration potential of $U=100 \mathrm{eV}$ and an average electronic current of $I=1 \mathrm{pA}$, the achievable brightness is of the order of $10^{2} \mathrm{~A} / \mathrm{Vm}^{2} \mathrm{sr}$, hence within the upper region of values from flat pulsed electron sources.

However, this estimate does not take into account that for a tip-geometry, the transverse momentum is correlated to the emission site, which leads to a smaller effective source size (see Sec. 3.4.2). Hence, the actual experimental brightness of such sources might be significantly higher.

\subsubsection{Temporal resolution}

The defining quantity of any time-resolved experiment is of course its achievable temporal resolution. Hence, a comparison between the planar and the tip emitter geometries, as sketched in Fig. 3.3, is undertaken based on a simple analytical model (For details, see App. A.1).

Specifically, the pulse duration $\tau_{\text {pulse }}$ of an electron pulse with energy spread $\Delta E$ and after propagation of a distance $d$ in the field of a plate capacitor with acceleration voltage $U$ can be expressed as

$$
\tau_{\text {pulse }}=\frac{\sqrt{2 m_{e}}}{e U} d\left(\sqrt{e U+E_{0}+\Delta E}-\sqrt{e U+E_{0}}-\sqrt{\Delta E}\right)
$$

with $m_{e}$ and $e$ the electron mass and charge, respectively, and $E_{0}$ the initial mean kinetic energy of the electron after the emission process. In the case of an electron accelerated from a nanometric tip under the same conditions, the pulse duration can be 
approximated as

$$
\tau_{\text {pulse }}=\sqrt{\frac{m_{e}}{2}} d\left(\frac{1}{\sqrt{e U+E_{0}+\Delta E}}-\frac{1}{\sqrt{e U+E_{0}}}\right) .
$$

Figure 3.6 displays the electron pulse durations in both geometries as a function of the initial energy spread for three different low electron energies. In the case of the flat cathode geometry (Fig. 3.6A), electrons are drawn from an emission area comparable to the size of the laser focus, which is usually a few tens of micrometers in diameter. Subsequent linear acceleration towards the anode (denoted with $(+)$ in Fig. 3.3A) leads to typical pulse durations in the range of few tens to more than hundred picoseconds after a propagation distance of $2 \mathrm{~mm}$ (Fig. 3.6A).
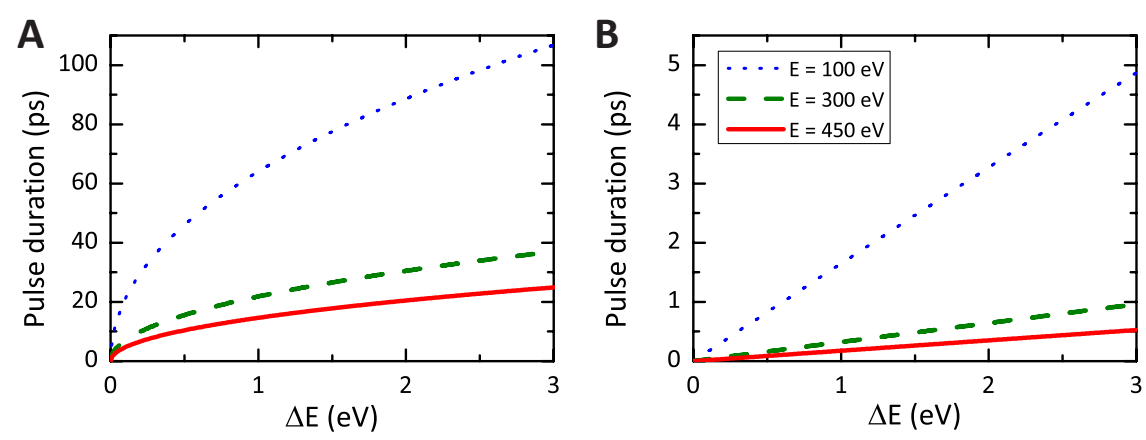

Figure 3.6: Estimated temporal resolution of $(\mathbf{A})$ a flat and $(\mathbf{B})$ a tip-based photocathode as a function of electron energy spread $\Delta E$. Solid red, dashed green, and dotted blue lines for final electron energies of $100 \mathrm{eV}, 300 \mathrm{eV}$, and $450 \mathrm{eV}$, respectively.

Propagation distance: $2 \mathrm{~mm}$. Model is described in detail in App. A.1.

In contrast, in the case of nanometric field emitters, nonlinear photoemission leads to selective emission from the tip apex (Fig. 3.6B). Electrons are then accelerated to velocities close to their final velocity within few tens of nanometers. This minimizes the propagation time and strongly suppresses dispersive pulse broadening, resulting in pulse durations more than 20 times shorter as compared to the model based on linear acceleration.

\subsection{Summary}

The concept of an electron source based on nonlinear photoemission from a sharp metal tip has been introduced. Subsequently, the characterizing quantities, namely emittance, brightness, coherence and temporal resolution have been estimated for such an electron gun within a time-resolved LEED experiment. 
It has been found that the average brightness of pulsed electron sources in general is strongly reduced in comparison to continuous emitters due to their extremely shot duty cycle. However, when applied within a stroboscopic approach, the brightness is of less importance than in the single shot mode, since it can be potentially compensated for by longer integration times. Therefore, a high brightness is mainly important for the fast acquisition of high quality images. Compared to flat state of the art photocathodes, a tipbased geometry offers comparable or slightly higher brightness at an reduced emittance, allowing for an increased focusability of the electron pulses. In terms of the achievable spatial resolution, the extremely localized area of emission of nanometric photocathodes leads to a high transverse coherence, making such sources ideal for the investigation of systems with large unit cells, e.g., complex organic molecules.

Most importantly, the achievable temporal resolution is strongly enhanced in comparison to capacitor-type geometries. This effect is especially pronounced at low energies: Electrons emitted from the tip surface are basically instantly accelerated to velocities very close to their final velocity, hence significantly reducing the time of flight and their susceptibility to dispersive broadening. Additionally, the tip-geometry is less susceptible to space-charge effects due to quickly diverging trajectories of emitted electrons.

In conclusion, nonlinear driven nanometric photocathodes offer excellent pulse properties in terms of achievable spatial and temporal resolution as well as overall current even at low electron energies, making them ideal candidates for the application in ultrafast imaging and diffraction experiments including ULEED. 



\section{Chapter 4}

\section{Numerical analysis of a tip-based}

\section{ultrafast electron gun}

In this section, a numerical study of an electron source with tip-based geometry is provided. First, a brief introduction to the employed finite element method (FEM) is given (Sec. 4.1). Next, the influence of the individual lens components with respect to focusability and temporal resolution of the electron pulse is simulated (Sec. 4.2). The chapter closes with the analysis of the impact of energy dispersion on the electron pulse duration (Sec. 4.4) and a discussion on the feasibility of the simulated approach (Sec. 4.5). Parts of the results presented here have been published in Ref. [52].

\subsection{Finite element simulation}

For the development of an ultrafast electron gun suitable for the operation at low electron energies, we theoretically model the source properties using a FEM-based approach. In particular, the spatio-temporal evolution of single short electron wave packets emitted from a nanoscopic photocathode are investigated. A more detailed account on the simulation procedure is given in App. A.3.

The finite element computations are performed in the MATLAB ${ }^{1}$ programming environment together with $\mathrm{GMSH}^{2}$ for the initial mesh generation. In order to reduce the

\footnotetext{
${ }^{1}$ The MATLAB code has been largely programmed by Felix Schenk. Information about MATLAB can be found at: http://www.mathworks.de/products/matlab/

${ }^{2}$ Free, open source, 3D mesh generator for use in FEM, more information at: http://geuz.org/gmsh/
} 
computational effort, cylindrical symmetry is assumed as well as zero out-of-plane momentum. The electron propagation is simulated classically by solving the equation of motion in the computed electric field for pulses containing only single electrons. Since a) the normal operation regime of such a gun features one to few tens of electrons per pulse (see Sec. 3.2), and b) the tip geometry strongly suppresses space charge effects, electron-electron interaction has been neglected. This simplification is supported by experimental findings in Ref. [50], where space-charge effects for a similar tip-based electron emitter were not observed up to some hundreds of electrons per pulse, albeit for low laser repetition rates. Additionally, it is assumed that the electron generation occurs instantaneously, which is a good approximation for a sub-100-femtosecond driving laser in combination with electron pulse durations of the order of few picoseconds.

The geometrical parameters from the sharp metal tip are taken from experimentally employed tips produced within the framework of earlier works [185, 186] (also see Sec. 5.1). Specifically, a tip apex radius of curvature of $25 \mathrm{~nm}$ is used throughout the simulations.

At the beginning of the computations, several different electrostatic lens assembly geometries and potential configurations were simulated. The geometry chosen was derived from TEM optics and consists of a lens system comprising four individual components (Fig. 4.1), namely suppressor (S), extractor (E), lens (L) and anode (A), in addition to the metal tip $(\mathrm{T})$. These TEM-type lens assemblies were found to deliver good results in terms of pulse duration and focal size, as confirmed in a later experimental realization. In the numerical calculations, the anode is always kept at ground potential; hence, the energy of electrons, which have left the lens system, is solely dependent on the (negative) tip potential.

An exemplary potential distribution along with the mesh grid for the geometry used in the following simulations is shown in Fig. 4.1 (upper and lower half, respectively). In addition to the lens assembly, the sample is labeled $(\mathrm{P})$ and is positioned at a distance of about $1.5 \mathrm{~mm}$ from the anode $(\mathrm{A})$.

Electron trajectories are computed for only one half of the geometry depicted in Fig. 4.1 and subsequently mirrored at the tip axis to obtain the full picture in agreement with the assumption of cylindrical symmetry. 


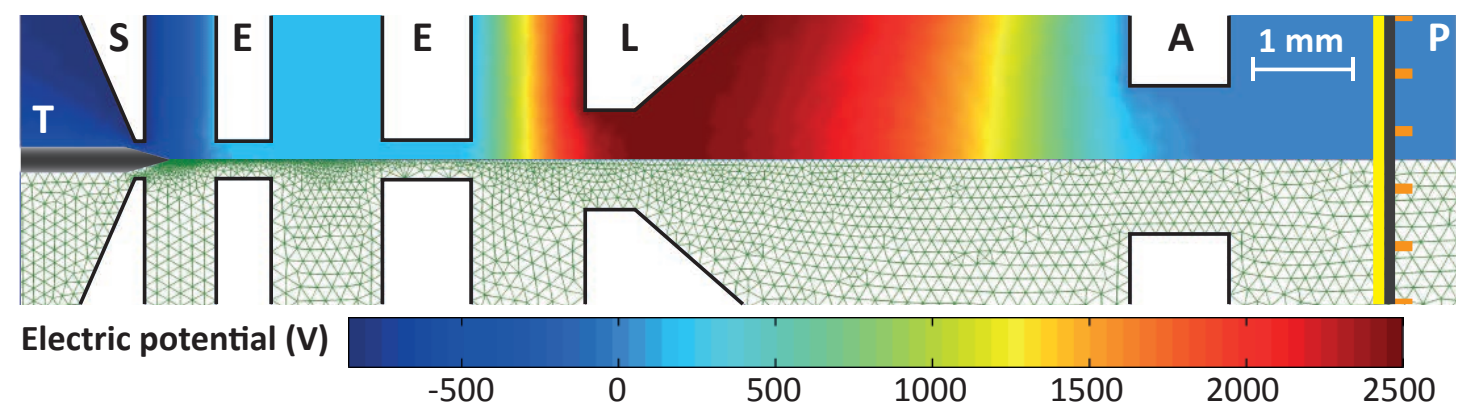

Figure 4.1: Electrostatic potential distribution (upper half) and mesh (lower half) computed for the ultrafast electron gun employed in the FEM simulations. T: tip, S: suppressor, E: extractor, L: lens, A: anode, P: sample.

The grid used in the FEM is manually adapted to the feature size of the surrounding geometry, as can be seen in Fig. 4.2. The possibility of refining the mesh size is of particular importance, since the evaluation of the electric potentials and fields has to be performed correctly and within a reasonable time interval not only in the vicinity of the nanometric sized tip (see Fig. 4.1A, lower half), but also for the macroscopic lens assembly. Before starting the simulations, the optimal temporal step width and mesh resolution have been determined.

\subsubsection{Energy and emission site distribution}

Since the exact spatial and temporal profile of photoemitted electrons from such nanometric photocathodes depends strongly on the detailed experimental conditions including, for example, the laser intensity and wavelength, the applied tip potential or the crystalline structure of the employed tip [50, 186-188], the following generalizations are made: $(i)$ The initial energy distribution of electrons emitted from the tip is assumed to be Gaussian

$$
D(E)=D_{0, E} \Theta(E) \exp \left(-\frac{8 \log (2)\left(E-E_{0}\right)^{2}}{2 \Delta E^{2}}\right),
$$

where $\Theta(E)$ is the Heaviside function ensuring only positive values of $E, D_{0, E}$ is a normalization factor, and $\Delta E$ is the full width at half maximum (FWHM) of the energy distribution width around the distribution center $E_{0}$. The factor of $8 \log (2)$ stems from the relationship between the standard deviation $\sigma$ and the FWHM of a Gaussian distribution: $\mathrm{FWHM}=2 \sqrt{2 \log (2)} \cdot \sigma \approx 2.35 \sigma$. For generality, all obtained values for the pulse duration and focal size are given in terms of the FWHM of their respective temporal and radial electron distributions. 

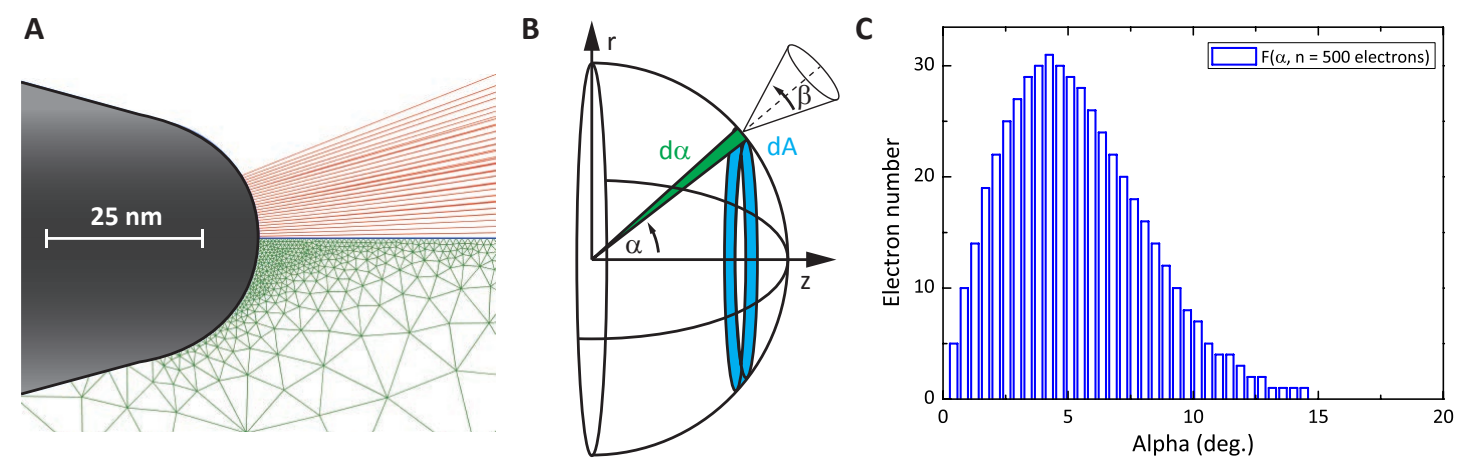

Figure 4.2: Simulated field emitter tip apex and angular electron distribution. A: FEM mesh (lower half) and trajectories (red) of emitted electrons (upper half). B: Emission site distribution takes into account three dimensionality of apex. C: Exemplary resulting angular distribution for $n=500$ electrons.

(ii) The electrons are assumed to be uniformly emitted into a (2D) solid angle of $\beta=45^{\circ}$ centered around the surface normal of the emission site. In a previous study on this system, $\beta$ was assumed to be zero [52]. (iii) The emission site distribution follows again a Gaussian distribution

$$
D(\alpha)=D_{0, \alpha} \exp \left(-\frac{8 \log (2)\left(\alpha-\alpha_{0}\right)^{2}}{2 \Delta \alpha^{2}}\right) \sin \alpha
$$

The term $\sin \alpha$ results from taking into account the three-dimensionality of the emission sites on the tip apex (Fig. 4.2B). In this case, the infinitesimal surface area becomes $d A(\alpha)=2 \pi r^{\prime} d \alpha=2 \pi(r \sin \alpha) d \alpha$ at a given angle $\alpha$. At first glance, this consideration might lead to a somewhat oddly shaped trajectory distribution with low density along the main symmetry axis, as for example seen in Fig. 4.3B. However, this assumption is necessary to correctly weight the tip surface in three dimensions, when assuming a constant emission density over the emitting area. An exemplary angular distribution is given in Fig. 4.2C.

In the strong-field study of Bormann et al. [50], a solid angle of emission of about $\Omega=$ $0.28 \mathrm{sr}$ was found in the multiphoton regime. For a conical approximation of the shape of the emission volume, this translates into a half-angle of $\alpha=\arccos \left(1-\frac{\Omega}{2 \pi}\right) \approx 17^{\circ}$.

\subsection{The suppressor / extractor unit}

In a first step, the influence of the suppressor unit on the emission characteristics of the gun is simulated. The suppressor unit is negative with respect to the tip bias potential 
and serves two main functions: Firstly, it suppresses electrons generated not from the apex, e.g., from hotspots ${ }^{3}$ along the tip shaft, and secondly, together with the extractor, it can be used to control the field enhancement at the apex (Fig. 4.3). For now, we keep the extractor voltage at a fixed value and change only the suppressor settings.
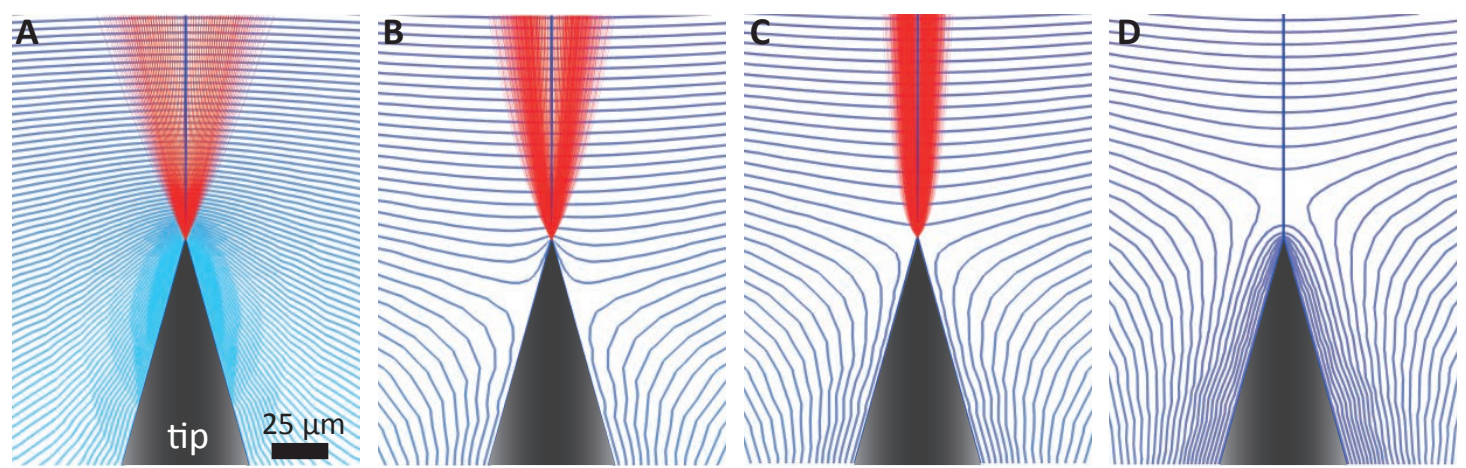

Figure 4.3: Influence of the suppressor on the field distribution and electron trajectories from the tip. Potentials are: tip: $-450 \mathrm{~V}$, extractor: $150 \mathrm{~V}$, lens and anode: $0 \mathrm{~V}$. Electron trajectories shown in red, equipotential contour lines in blue, step size: $5 \mathrm{~V}$. A: Suppressor potential $U_{S}=-550 \mathrm{~V}$, relatively close to the tip potential. Strongly diverging electron trajectories, strong field enhancement. B: $U_{S}=-860 \mathrm{~V}$, electron trajectories less divergent, lower field enhancement. C: $U_{S}=-923 \mathrm{~V}$, electron trajectories now strongly collimated, nearly complete suppression of field enhancement. D: Electron emission cut off due to field reversal at the tip apex, shown for $U_{S}=-1100 \mathrm{~V}$.

For suppressor voltages $U_{S}$ close to that of the tip, electrons propagate away from their emission site normal to the tip surface, leading to a strongly divergent beam (Fig. 4.3A). In order to avoid pulse broadening resulting from different path lengths of electrons from different emission sites, the suppressor voltage can be decreased (Fig. 4.3B). This eventually leads to an increasingly pronounced suppression of the field enhancement around the tip (Fig. 4.3C) and finally to a field reversal (Fig. 4.3D), resulting in the complete extinction of electron emission. The reduction of the field enhancement also decreases the acceleration of the electrons, ultimately leading to a capacitor-like potential distribution at the cut-off voltage $\left(U_{S}=-924 \mathrm{~V}\right.$, situation similar to Fig. $\left.4.3 \mathrm{C}\right)$ and hence an increased electron pulse duration (Fig. 3.6B). Therefore, the optimal suppressor setting will be a tradeoff between two regimes: Path-length-induced temporal broadening on the one side and broadening caused by reduced field enhancement at the tip apex on the other side. Additionally, it is expected that the optimal suppressor potential shifts closer to the tip potential, when the suppression of dispersive broadening (and hence a stronger field enhancement) becomes important. This outcome is the case for a large electron energy distribution width $\Delta E[52]$.

\footnotetext{
${ }^{3}$ Hotspots are regions on the tip shaft with radius of curvature comparable to the apex, leading to strong electron emission within the tip area, which is illuminated by the driving laser.
} 
A

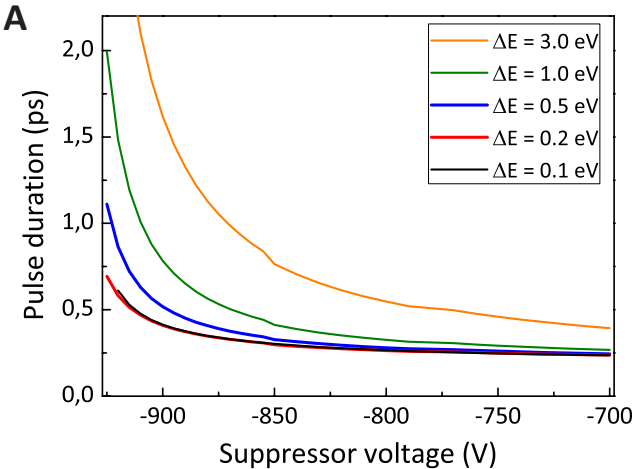

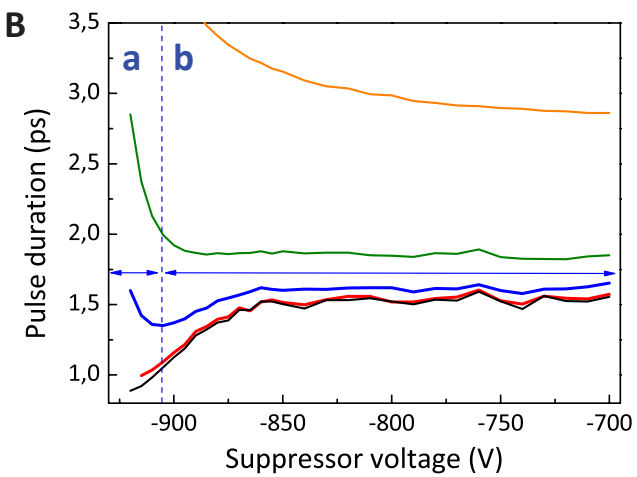

FigURE 4.4: Influence of the suppressor voltage on the pulse duration as a function of electron energy spread $\Delta E$ for two different propagation distances (A: $0.5 \mathrm{~mm}, \mathbf{B}: 12$ $\mathrm{mm}$ ). Dashed line in B indicates the optimal suppressor setting for $\Delta E=0.5 \mathrm{eV}$ (blue solid line): pulse broadening effect due to (a) reduced field enhancement and (b) path length differences. Tip voltage: $-450 \mathrm{~V}$.

The dependence of the pulse duration on the suppressor voltage as a function of $\Delta E$ is simulated in Fig. 4.4A after propagation from the apex to the extractor $(d=0.5 \mathrm{~mm})$. It displays a growing temporal pulse width by $(i)$ a decrease of the suppressor voltage and $(i i)$ an increase of the energetic spread of the electron energy distribution. As expected, the impact of the suppressor voltage setting rises when assuming broader energy distributions, since in this case, field enhancement becomes more crucial for short electron pulses. Yet, pulse broadening due to path length-variations cannot be observed in Fig. 4.4A, since the propagation distance is too small (effect visible in Fig. 4.4B and in Ref. [52], Fig. 7).

Figure 4.4B displays the pulse duration as a function of suppressor voltage with the same settings as in Fig. 4.4A but at a significantly longer propagation distance of $12 \mathrm{~mm}$, corresponding to the full distance to the sample position. For a relatively narrow range of electron energy widths, a suppressor voltage close to the cut-off threshold is favorable, resulting in strong radial compression of the pulse $(\Delta E \leq 0.5 \mathrm{eV})$. Upon enlargement of $\Delta E$, the effect of velocity dispersion becomes visible by strongly increased temporal broadening for the aforementioned suppressor settings (region denoted by "a" for $\Delta E=$ $0.5 \mathrm{eV})$. On the other hand, pulse lengthening is caused due to path-length differences at more positive suppressor settings (region denoted by "b" for $\Delta E=0.5 \mathrm{eV}$ ).

Qualitatively, the optimal suppressor voltage for a minimal pulse duration in this setup increases with a broader electron energy spectrum. This can be understood by the growing importance of field enhancement to compensate dispersive broadening. In this 
simulation, however, the effect of the extractor and the lens, which themselves have strong influence on tempo-spatial pulse development, have not yet been taken into account. These components as well as their interplay will therefore be analyzed in the following.

A control of the initial acceleration of the electrons away from the tip by means of the extractor voltage is especially useful at low electron energies to avoid pulse broadening from long overall propagation times. Figure 4.5 displays the temporal spread of the electron pulse after $2 \mathrm{~mm}$ of propagation for different extractor voltages and a tip potential of $-100 \mathrm{~V}$. The extractor and suppressor settings are chosen to yield the same potential at the position of the tip apex, only the gradient (the field) is changed. As expected for an increasing extraction potential, the temporal broadening is significantly suppressed. Within an experimental realization, the extractor potential is of course somewhat restricted due to the danger of electric sparkovers. We therefore use potential distributions with a maximum electric field strength of about $3.0 \mathrm{kV} / \mathrm{mm}$ between suppressor and extractor to avoid the mentioned sparkovers as well as static field emission from the sharp tip.

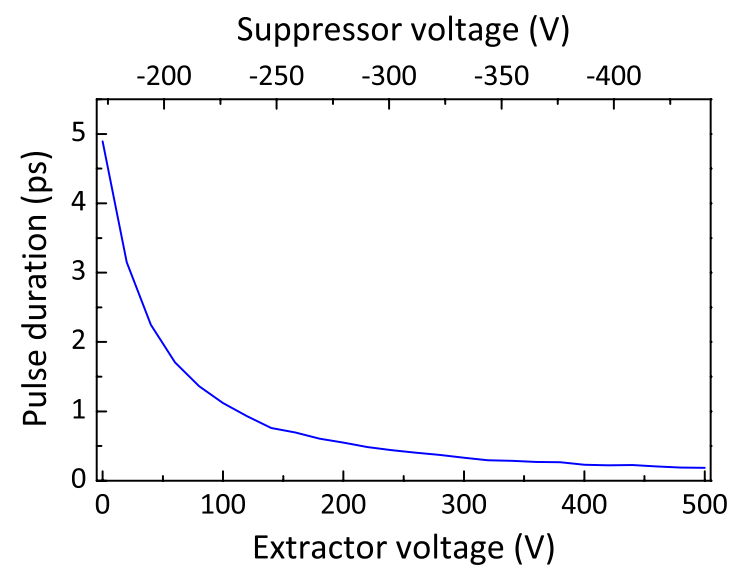

Figure 4.5: Pulse duration as a function of extractor voltage. Tip potential: $-100 \mathrm{~V}$. Propagation distance: $2 \mathrm{~mm}$. Electrons per data point: 100. Energy spread: $1 \mathrm{eV}$.

\subsection{The lens: pulse duration, focusability, and total cur- rent}

In a next step, the pulse duration as a function of lens voltage is simulated for different suppressor voltages to find the respective optimal operation regime of the lens. For this 
simulation, a sample is assumed to be at a position about $1.5 \mathrm{~mm}$ after the anode, at a total distance of $12 \mathrm{~mm}$ away from the tip apex. The tip and extractor potentials are held at $-450 \mathrm{~V}$ and $150 \mathrm{~V}$, respectively, while the lens potential is scanned for a fixed suppressor voltage. For each point, the trajectories of 4500 electrons with an initial kinetic energy spread of $\Delta E=1 \mathrm{eV}$ around an offset of $E_{0}=1.5 \mathrm{eV}$ and an angular distribution with $\alpha=10^{\circ}$ and $\beta=45^{\circ}$ are used. The solid angle of emission $\alpha$ was chosen to be somewhat smaller than experimentally found in Bormann et al. [50] and instead be in accordance to the value used in the joint publication with A. Paarmann [52] for better comparison (see Sec. 4.5).

The pulse duration exhibits a local minimum close to the ground potential and a global minimum at lens voltages around $2500 \mathrm{~V}$ (Fig. 4.6A). With increasing suppressor voltage (closer to the tip potential), the pulse duration becomes smaller and the minima more pronounced. A minimal pulse duration is achieved for $U_{L}=-2450 \mathrm{~V}$ and $U_{S}=-700 \mathrm{~V}$.
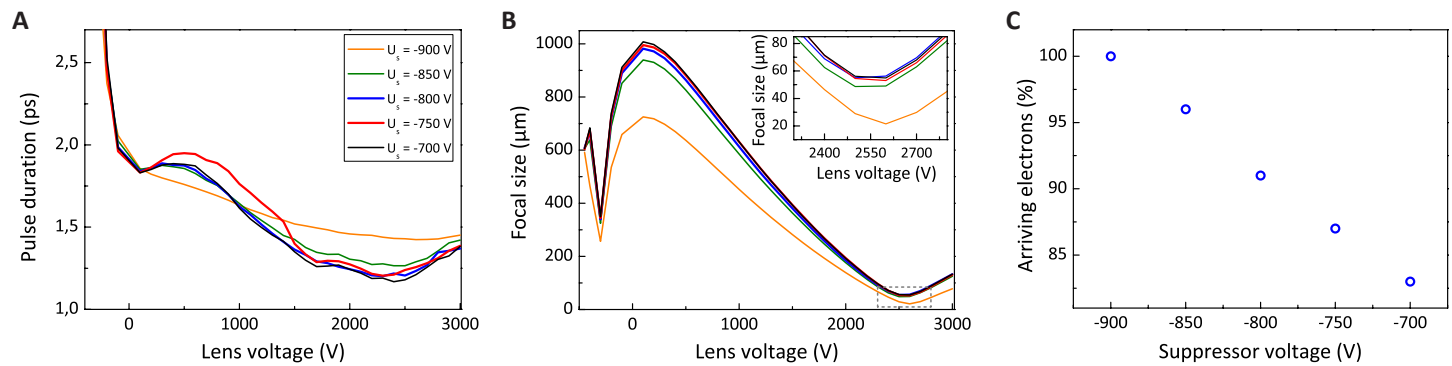

FIgURE 4.6: Simulation of the lens and suppressor influence on pulse duration, focal width and throughput. Potentials: tip: $-450 \mathrm{~V}$, extractor: $150 \mathrm{~V}$. Propagation distance: $12 \mathrm{~mm}$. Energy spread: $1 \mathrm{eV}$. A: Pulse duration as a function of lens voltage for different suppressor voltages. B: Radial spread of arriving electrons. Inset: Magnified region denoted by gray dashed rectangle. C: Relative amount of arriving electrons at the sample position $(12 \mathrm{~mm})$.

The radial electron pulse spread is displayed in Fig. 4.6B. Again, two minima exist, one local minima at lens voltages close to the tip potential and a global one between lens voltages of $2500 \mathrm{~V}$ and $2600 \mathrm{~V}$. The smallest focus is achieved for a suppressor voltage of $-900 \mathrm{~V}$ and a lens voltage of $2580 \mathrm{~V}$ (Fig. 4.6B, inset). Further minima are observed at higher lens voltages $(>5000 \mathrm{~V})$, which are neglected, since they $(i)$ do not offer better beam characteristics in terms of temporal and spatial spread and (ii) are technically more difficult to realize, again due to the increasing danger of electric sparkovers at high voltages.

Both the focal spread and the pulse duration exhibit their minima under comparable conditions. In particular, the second minimum around a lens voltage of $2500 \mathrm{~V}$ is 
displaying a relatively small electron focus $(20-60 \mu \mathrm{m})$ as well as pulse durations of the order of 1.2-1.5 ps. Both minima in pulse duration result from a (local) minimization of the path length differences, naturally best achieved close to the lens settings for minimal radial spread.

For a suppressor voltage close to the cutoff voltage (Fig. 4.3D), the influence of the lens on the pulse duration is somewhat reduced compared to more positive suppressor voltages. This behavior stems from the focusing property of the suppressor and can lead to the shortest possible electron pulses for very small $\Delta E$. In the present case as shown in Fig. 4.6, the energy spread is taken to be $\Delta E=1 \mathrm{eV}$, therefore resulting in longer pulse durations for $U_{S}=-900 \mathrm{~V}$ as compared to less negative suppressor settings.

In general, a suppressor voltage close to the tip potential is beneficial in terms of minimal achievable pulse duration, while slightly increasing the focal size. The enlarged focus can be explained by analyzing the influence of the solid angle of emission ( $\beta$-parameter) at different suppressor settings. In the case of reduced field enhancement, electron emitted at the surface are subject to the capacitor-like potential distribution between extractor and suppressor almost immediately. A strong bending of the electron trajectories in the forward direction results in a reduction of the effective source size. At higher field enhancements, the emission direction of electrons is initially preserved, leading to an increased effective source size. However, the overall impact of the suppressor setting is relatively small when operated sufficiently away from the cutoff voltage, which leaves a large parameter space of possible operation voltages.

When choosing the optimal operation regime of the gun, an additional parameter to consider is the absolute throughput of electrons. The throughput is the percentage of electrons emitted from the tip, which actually arrive at the sample position (Fig. 4.6C). The losses are mainly a result from electrons blocked by the first and in a minor degree the second extractor aperture, when operating the gun with a more divergent beam. In the simulations, the total loss amounts to about $10 \%$ at $U_{S}=-800 \mathrm{~V}$. This somewhat limits the useful operational regime of the suppressor, which will now be a tradeoff between the maximal achievable temporal resolution on the one hand and a sufficient signal intensity on the other hand. Moreover, it has to be taken into account that within the simulation, an ideal alignment of the tip and lens elements with respect to each other is achieved, which is most likely not the case in an experimental realization. 
In the latter, electron losses will therefore be larger, ultimately limiting the range of suppressor settings.

As for the lens voltage, the optimal setting is straight forward: There is only one pronounced minimum in the spatial as well as in the temporal spread, which is achieved at comparable lens voltages.

\subsection{Energy dispersion}

The above simulation was performed for a single energy spread of $\Delta E=1 \mathrm{eV}$. Now, the influence of $\Delta E$ on the achievable spatio-temporal resolution is determined for a fixed suppressor voltage of $U_{S}=-860 \mathrm{~V}$ (Fig. 4.7). This voltage is chosen while trying to satisfy the above mentioned criteria for an optimal operation regime. In terms of angular distribution of the emission sites, lens voltages and total number of electrons simulated per data point, the simulation parameters are kept equal to those used in the analysis of the influence of the suppressor settings (Fig. 4.6).
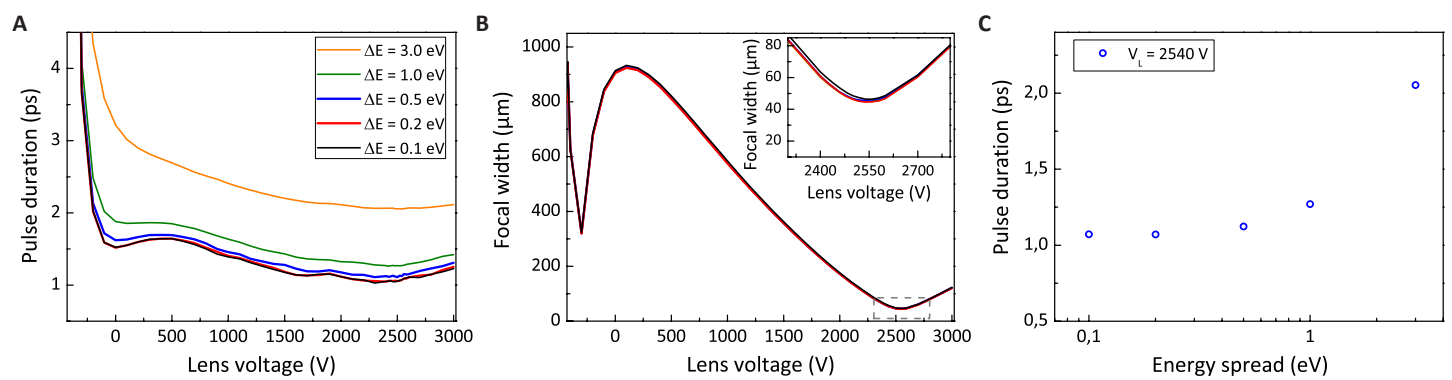

FIGURE 4.7: Influence of the initial electron energy spread $\Delta E$. Applied potentials: tip: $-450 \mathrm{~V}$, suppressor: $-860 \mathrm{~V}$, extractor: $150 \mathrm{~V}$. Lens voltage dependent pulse duration $(\mathbf{A})$ and focal size $(\mathbf{B})$ as a function of $\Delta E$. Inset in $\mathrm{B}$ is a magnification of area denoted by dashed grey rectangle. C: Pulse duration at lens setting for smallest focus as function of $\Delta E$.

Figure 4.7A displays the pulse duration as a function of lens voltage for initial electron energy distributions widths of $0.1 \mathrm{eV} \leq \Delta E \leq 3.0 \mathrm{eV}$. The behavior displays the two minima as described in Fig. 4.6A, except for very high $\Delta E$. For moderate to small energy spreads, the minimal achievable pulse duration within the chosen geometry is found to be between $1.0 \mathrm{ps}$ and $1.3 \mathrm{ps}$. Within the simulated accuracy, no influence of $\Delta E$ on the focusability of the electron pulses at the sample position has been observed. Lens voltage settings between $2300 \mathrm{~V}$ and $2800 \mathrm{~V}$ seem to offer good focusability of the beam down to about $50 \mu \mathrm{m}$. These values are of course a strong function of the 
initial angular distribution of the emission sites as well as of the direction of emission of the electrons, where especially the former has a significantly influence on the focal size. For comparison, in the simulations performed together with A. Paarman [52], we find standard beam deviations between $25 \mu \mathrm{m}$ and $50 \mu \mathrm{m}$ for a similar tip voltage of $-400 \mathrm{~V}$, taking into account, however, only emission normal to the tip surface $(\beta=0)$. If we assume a Gaussian distribution of the radial coordinate of the electrons at the sample position, the standard deviation translates to the FWHM by multiplication with a factor of $2 \sqrt{2 \log (2)} \approx 2.35$, thus resulting in values similar or even slightly bigger than those in the present work for a comparable angular distribution. Considering that the effective source size is basically zero in the case of $\beta=0$, a significantly smaller focus than in the present work could have been expected, since the simulated systems were otherwise comparable. At this moment it remains unclear to what extent the focusability of the electron beam is influenced by the distribution of the initial electron velocity vector and the individual components of the electrostatic lens system.

The pulse duration as a function of the initial spread $\Delta E$ is displayed in Fig. 4.7C at a given lens voltage of $U_{L}=2550 \mathrm{~V}$. The graph displays a steep increase of the pulse duration for $\Delta E>1.0 \mathrm{eV}$ for this suppressor setting. Hence, the influence of $\Delta E$ on the optimal suppressor setting has to be kept in mind for an experimental realization of such an electron source.

Finally, a strong influence of the tip radius on the pulse duration is not found for a rather large range of tip radii, an observation which is somewhat parallel to the broad operation regime of the suppressor: A larger tip radius would lead to a reduced field enhancement, which does not have a huge impact within a certain range, as seen from the calculations in Fig. 4.4, assuming an initial electron energy distribution width $\Delta E$ of not more than 1 or $2 \mathrm{eV}$. Furthermore, since the suppressor unit can basically tune the field enhancement at the tip apex by controlling the surrounding potential distribution, changes in the tip radius can easily be balanced out within a reasonable range of values.

\subsection{Summary and discussion}

In comparison to the results of the joint study with A. Paarmann [52], significantly longer pulse durations have been computed at comparable lens geometries, energies and 
energetic spreads. This can be mainly attributed to the following reasons: Firstly, the propagation distance in Ref. [52] has been chosen to be only about $8 \mathrm{~mm}$ in comparison to $12 \mathrm{~mm}$ used here. As seen in Eq. 3.13, the pulse duration increases roughly linearly with the propagation distance. On the other hand, the study presented here uses higher energy electrons, $450 \mathrm{eV}$ compared to $300 \mathrm{eV}$, but at lower extraction fields (150 V compared up to $600 \mathrm{~V})$.

The main difference, however, comes from the inclusion of non-normal emission from the tip surface. By allowing for $\beta>0^{\circ}$, not only the effective source size increases (and therefore the minimal achievable focal size), but also the effect of dispersive broadening. A higher initial kinetic energy offset of $E_{0}=1.5 \mathrm{eV}\left(E_{0}=0.5 \mathrm{eV}\right.$ in Paarmann et al.) further increases this tendency ${ }^{4}$. Compared to the narrow energy distribution of $\Delta E=0.2 \mathrm{eV}$ as chosen in the joint study, this leads to a strong increase in pulse duration. However, a non-zero $\beta$ is a more realistic assumption compared to emission exclusively normal to the tip surface [156] and should result in good agreement with experimentally recorded values (see Sec. 5.3.2).

In conclusion, the above performed simulations demonstrate the possibility to operate laser-driven nanoscopically sharp metal tips as ultrafast electron guns. Such electron sources are potentially suitable but not limited to time-resolved low-energy applications as ULEED. As a rough guideline for an optimal performance,

- the width of the initial kinetic energy spectrum of the emitted electrons should be kept as small as possible, ideally below $\Delta E=1.0 \mathrm{eV}$ (Fig. 4.7C),

- a combination of a strong intermediate acceleration by the lens and subsequent deceleration by the anode offers the smallest pulse durations in the regime of $1 \mathrm{ps}$ together with good beam collimation of the order of $50 \mu \mathrm{m}$ (Fig. 4.7),

- the suppressor should be operated in a regime not too close to the cutoff voltage to avoid pulse broadening by loss of field enhancement and not too close to the tip potential to avoid a significant electron current decrease at the apertures (Fig. 4.4),

- the effect of temporal broadening due to path length differences is only visible for very narrow energy distributions and can in most cases be neglected,

\footnotetext{
${ }^{4}$ As will be seen in the intensity-dependent electron current measurement (Sec. 5.3.1), an initial energy of $E_{0}=1.5 \mathrm{eV}$, corresponding to half the photon energy of $400 \mathrm{~nm}$ laser pulses, models the emission process more realistically.
} 
- a relatively high extractor voltage should be chosen, especially at low electron energies, to increase the initial acceleration of the electrons (Fig. 4.5).

It should be noted that the operation regime of such a gun is not limited to the use of low-energy electrons, but could be readily implemented in a similar fashion within various other time-resolved imaging and diffraction experiments. 



\section{Chapter 5}

\section{Experimental analysis of a tip-based ultrafast electron gun}

In this chapter, the experimental realization of the simulated gun design is presented, starting with the preparation procedure of the needle emitter (Sec. 5.1). Furthermore, the setup of the ULEED experiment is described (Sec. 5.2) and a characterization of key parameters such as pulse duration is given (Sec. 5.3). Finally, the electron source is compared with the simulation of the previous chapter and possible improvements are discussed (Sec. 5.4). The chapter ends with a brief discussion on an electron source operated in the optical field emission regime (Sec. 5.4.3), THz compression of the electron pulses (Sec. 5.4.4), as well as alternative methods for a more precise determination of the pulse duration (Sec. 5.4.1).

\subsection{Tip preparation}

For the realization of an electron source as employed in the simulations, nanometric sharp tungsten tips are produced by chemical wet etching in a procedure derived from a tip-production scheme for STM tips as depicted in Fig. 5.1 [189]. Specifically, a tungsten ${ }^{1}$ wire of $250 \mu \mathrm{m}$ radius is tapered by electrochemical polishing in an $3.5 \%$ mol aqueous $\mathrm{KOH}$ solution for 8-10 minutes with an applied DC voltage of 6.5 V. Etching proceeds, until the weight of the part submerged in the etching solution cannot be supported by

\footnotetext{
${ }^{1}$ Vendor: GoodFellow (http://www.goodfellow.com/), purity: $99.95 \%$, order number: W 005150.
} 
the notched region at the solution / air interface and falls to the bottom of the beaker. At this point, the etching current $I$ drops and the etching is stopped by electronically shutting off the external voltage. Tungsten tips are then washed in ethanol and deionized (DI) water and routinely characterized in terms of straightness, smoothness and radius of curvature under the optical microscope and subsequently by a SEM (Fig. 5.2C).

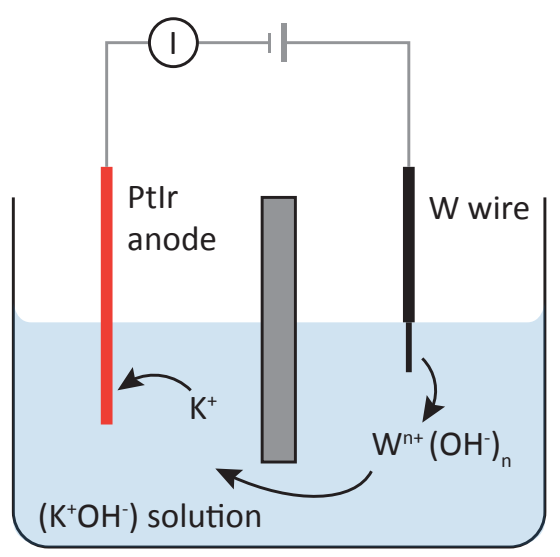

FiguRE 5.1: Sketch of chemical wet etching process of sharp tungsten tip preparation.

\subsubsection{Tip alignment}

In a next step, the tungsten tip is mounted into the suppressor unit as shown in Fig. 5.2.
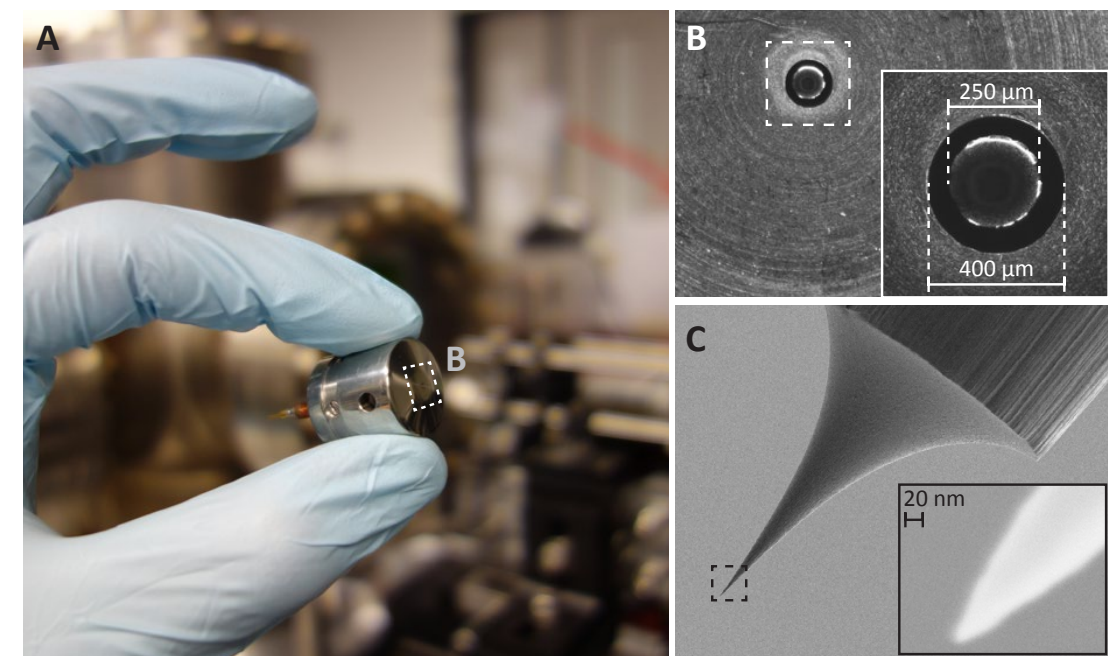

Figure 5.2: Nanometric photocathode and suppressor unit. A: Photograph of the suppressor unit as used within the electrostatic lens assembly. Dashed white box denotes region shown in B. B: Top-down view of the suppressor unit and tungsten tip. Inset: Magnified area as indicated by white dashed box. C: SEM image of the tungsten tip used, inset shows radius of curvature of about $10 \mathrm{~nm}$.

The exact alignment of the tip within the suppressor aperture is performed manually under an optical microscope and requires experience and patience. It is found that a 
correct alignment is essential to avoid blocking a large part of the electron beam by the apertures of the lens assembly (see also Sec. 4.3).

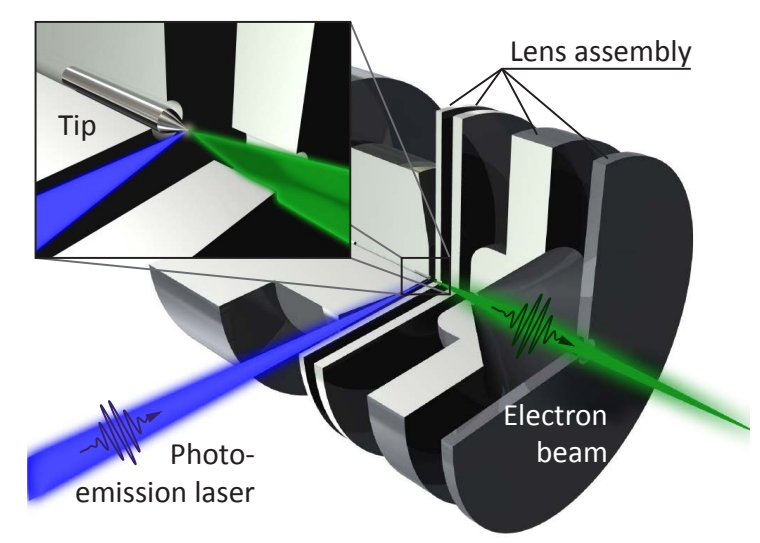

Figure 5.3: Conceptual drawing of the ultrafast electron gun. Inset displays magnified version of tip region denoted by dark rectangle.

The suppressor unit with tungsten tip is subsequently aligned within the lens system as shown in a conceptual drawing of the assembled gun in Fig. 5.3. To avoid the high electric fields from the lens to alter electron trajectories outside of the gun, the whole gun is mounted within a shielding Faraday cup, which also comprises the anode (at ground potential) of the lens system. The total propagation distance within the lens system from the tip apex to the outside of the aperture is taken to be $12 \mathrm{~mm}$. The experimental setup in which the developed electron gun is employed is described in the next section.

\subsection{ULEED experimental setup}

The ultrafast electron gun is employed in a laser-pump / electron probe scheme as illustrated in Fig. 5.4. The amplified laser source, which delivers the pulses for sample excitation (pump) as well as for photoemission, comprises a Coherent Mantis Ti:sapphire oscillator ( $80 \mathrm{MHz}$ repetition rate, $7 \mathrm{~nJ}$ pulse energy, center wavelength $800 \mathrm{~nm}$, sub-100fs pulses) in combination with a Coherent RegA 9050 regenerative amplifier (variable repetition rate up to $312 \mathrm{kHz}, 5 \mu \mathrm{J}$ pulse energy, center wavelength $800 \mathrm{~nm},<80$ fs pulse duration). About half of the intensity from the amplifier is guided through an optical chopper and over a variable delay stage into the vacuum chamber to the sample (pump pulse, red). The other half is frequency-doubled via second-harmonic generation (SHG) in a $\beta$-barium borate $(\mathrm{BBO})$ crystal and focused on the tungsten tip (photoemission 
pulse, purple) by a movable lens outside of the vacuum chamber with $250 \mathrm{~mm}$ focal length. Before the lens, the laser beam is expanded to a diameter of about $25 \mathrm{~mm}$ by a telescope comprising two concave silver mirrors. The system of electron source, sample and detector assembly is operated under ultrahigh vacuum conditions $\left(10^{-8} \mathrm{mbar}\right.$ $>p>11^{-9}$ mbar) to avoid sample contamination as well as electron scattering on the way to the sample. A magnified sketch of the gun-sample-detector configuration is shown in Fig. 5.4B.

A

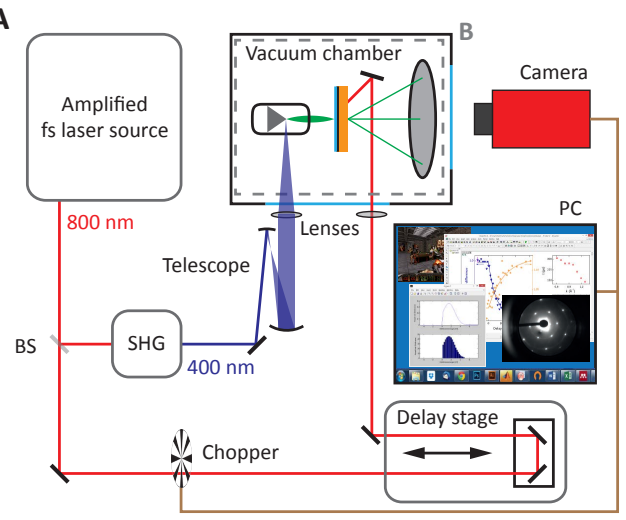

B

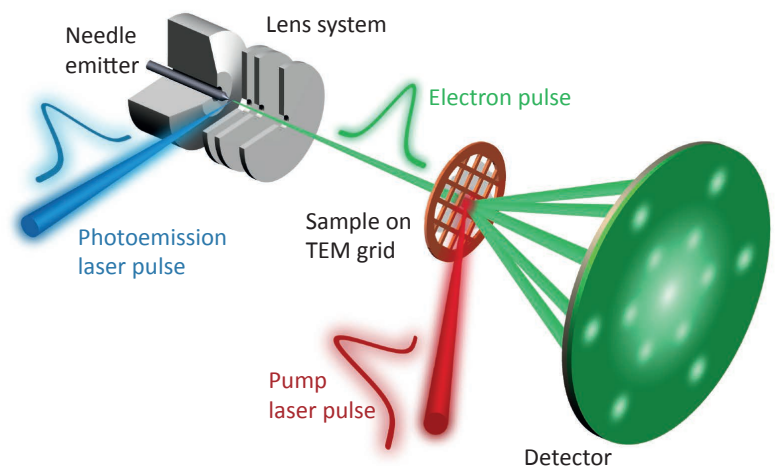

Figure 5.4: Experimental setup of ULEED. A: Schematic of the laser-pump / electron-probe setup. Dashed gray box denotes area magnified in B. B: Schematic of the gun-sample-detector system within the vacuum chamber (not to scale). [190]

Electrons emitted from the gun (green) arrive at the sample, which is situated on a TEM mesh grid. Depending on the operation mode of the setup, the electrostatic lens system is employed to produce either a focused or a divergent beam at the sample position. The excitation of the sample by the pump pulse (red) takes place from the backside to allow for a small incident angle as well as a reduced distance between electron source and sample. Scattered electrons are recorded on a phosphor screen microchannel plate (MCP) detector (model: Hamamatsu F2226-24P). The distance between the anode of the electron gun and the sample is about $2 \mathrm{~mm}$, resulting in a total propagation length of ca. $14 \mathrm{~mm}$ along the $\mathrm{z}$ axis from the tip apex to the sample ${ }^{2}$, while the sample-detector distance is set to about $90 \mathrm{~mm}$. A sketched overview of the vacuum chamber system from the experimental setup used is displayed in Fig. 5.5.

In contrast to classic LEED geometries (Sec. 2.1.2), the ULEED setup is currently operated in transmission. As will be demonstrated later (Chap. 7), diffraction recorded in a back-reflection geometry is also possible, but for the characterization of the electron source and the sample system in this work, a transmission setup is advantageous.

\footnotetext{
${ }^{2}$ Which is slightly longer compared to the value used in the simulation (12 mm).
} 


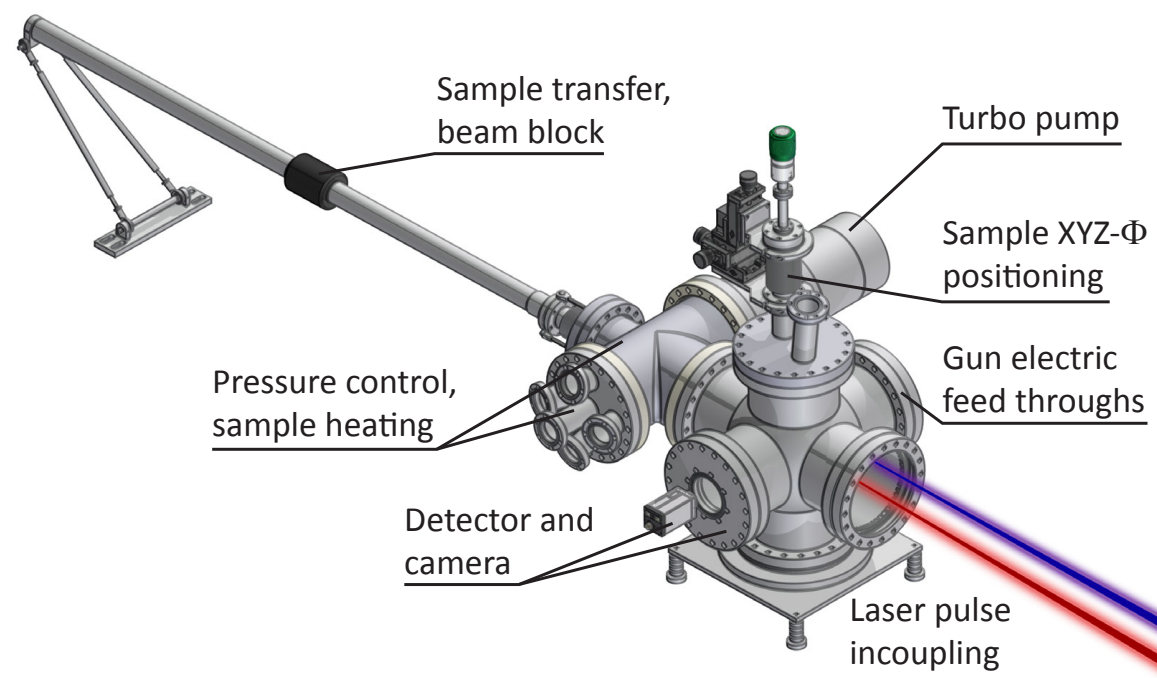

FiguRE 5.5: Sketch of the ULEED ultrahigh vacuum chamber system. Pump and photoemission pulses are indicated by red and purple rays, respectively.

A further difference with respect to a regular LEED is the use of a planar instead of a spherical-cap type detector. While a conversion from the planar to the spherical screen geometry is performed in the analysis, individual diffraction images presented in this work are shown as recorded.

\subsection{Experimental characterization of the ultrafast electron gun}

In this section, the ultrafast needle emitter is characterized with respect to its electron energy spectrum, the electron current and, most importantly, the achievable temporal resolution.

\subsubsection{Focal size, energy spectrum and brightness}

The focal size of the electron beam at the sample position can be determined, for example, by a knife-edge-type experimental configuration, as performed for this setup by S. Schweda in Ref. [191] (electron beam diameter at sample position: 60(5) $\mu \mathrm{m}$ ). Alternatively, the electrostatic lens of the electron source can be employed to zoom into the projection images of TEM grids with known mesh size. We found that for the setup described above, the electron beam can be focused through a single mesh cell of a 300 
mesh TEM grid ${ }^{3}$, hence yielding a minimal radial spread close to the values extracted from the simulation (Sec. 4.3) as well as from the knife-edge measurement.

For the determination of the electron energy spectrum, a retarding-potential energy filter is placed between the electron source and the detector. The differential electron intensity recorded on the detector peaks at the set kinetic electron energy and results in a spectral width of about $1.2 \mathrm{eV}$ FWHM, assuming a Gaussian energy distribution of the electrons (Fig. 5.6A). This measurement is mainly limited by the poor resolution of the A/D-converter PCI board that controls the programmable power supplies of the tip, the lens assembly and the energy filter. The resolution of the board in combination with the employed power supplies is of the order of $0.5-1.0 \mathrm{eV}$. Also, it has to be taken into account that only the energy component in the z-direction (along the tip axis) can be measured. Furthermore, the waviness of the employed grids limit the relative accuracy of the filter to about $2 \%$ [191].

A

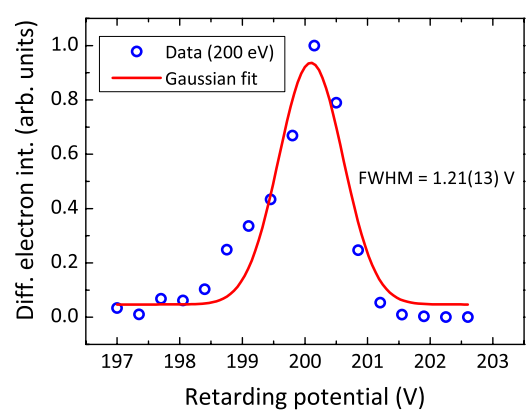

B

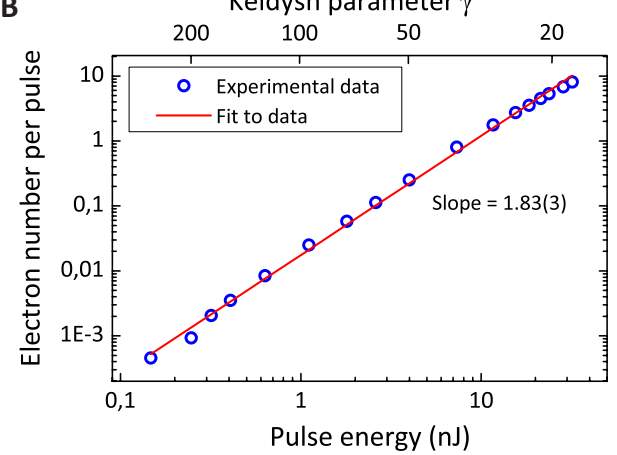

Figure 5.6: Characterization of the electron source used in the experiment. A: Electron energy distribution width at a pulse energy of $200 \mathrm{eV}$. B: Recorded electrons per pulse yielding a power law with exponent 1.8. Electron energy: $300 \mathrm{eV}$. Integration time: $1 \mathrm{~s}$. Detector quantum efficiency: $50 \%$. Electron loss before detector: $50 \%$.

Driving laser repetition rate: $312 \mathrm{kHz}$. Keldysh parameter as defined in Eq. 3.2.

Alternatively, an estimate of the energy spread can be made from a consideration of the photoemission process with respect to the material's work function. Depending on the surface direction at the tip apex, tungsten has work functions between $4.5 \mathrm{eV}$ (111) and $5.3 \mathrm{eV}$ (110) [192]. Assuming two-photon photoemission ((Fig. 3.2) from the tip with a single photon energy of $3.1 \mathrm{eV}$ (400 nm, see Sec. 5.2), excess energies in the order of $0.9-1.7 \mathrm{eV}$ are to be expected. However, this is not taking into account possible further work function modifications by oxidation of the tungsten [193] and applied potentials [172]. Since the tips are etched from polycrystalline tungsten, this would result in a

\footnotetext{
${ }^{3}$ Vendor: Plano GmbH (http://www.plano-em.de/); single mesh size: $70 \mu \mathrm{m}$. The mesh number gives the number of mesh cells per inch. The bar size is usually of the order of $5-15 \mu \mathrm{m}$.
} 
width of the energy spectrum of about $1.3 \mathrm{eV}$ for a uniform distribution of crystal surface directions.

Indirectly, an upper limit for the energy spread can also be obtained by a comparison of the measured pulse durations with the simulated ones. This would result in an energy spread of $1.15 \mathrm{eV}$ (App. A.1). For simplicity and until measurements with higher accuracy are undertaken, we presume an energy spread of approximately $1.0 \mathrm{eV}$. Beyond that, methods for the reduction of the energy spread are discussed at the end of this chapter.

So far, the claimed two-photon photoemission process driving the electron emission has not yet been experimentally confirmed. This hypothesis can be tested by measuring the electron current dependence on the photoemission laser pulse energy. The functional relation found is displayed in a double logarithmic plot in Fig. 5.6B. Specifically, the measurement is performed by recording the light intensity on the detector in the area of electron signal from the electron gun over $1 \mathrm{~s}$ for a given pulse energy. At very low emission currents, the detected electrons are counted manually as well as by using a counting algorithm [186], while at the same time recording their averaged intensity. This is followed by an estimation of the brightness of a single electron to extrapolate the electron count at higher emission currents. Finally, the relation was corrected for the detector quantum efficiency (taken as $50 \%$ ) and electron losses before the detector $(50 \%)$. As a result of a probably slight misalignment of the tip with respect to the center of the suppressor, the losses were taken to be higher than expected from the simulation for the given lens settings (Fig. 4.6C).

The functional dependence of the electron number per pulse $N_{e}$ on the pulse energy $E_{p}$ was found to follow the power law

$$
N_{e}=0.0175(2) \cdot\left(\frac{E_{p}}{\mathrm{~nJ}}\right)^{1.83(3)}
$$

With a maximum value of about 10 electrons per pulse at $E_{p}=30 \mathrm{~nJ}$ pulse energy. Compared to the experimental data on the power dependence by Bormann et al. [50], the employed pulse energies are significantly higher. This stems mainly from the larger focal size of the laser spot on the tip apex, which was about 10 times bigger in our experiment, resulting in a 100 times smaller intensity at the same pulse energies (see 
Keldysh parameter scale in Fig. 5.6B). When this is taken into account, the resulting electron numbers are comparable in magnitude.

The exponential given in Eq. 5.1 is reduced by about $10 \%$ compared to the exponential expected for a pure two-photon photoemission process. However, we have to consider a few effects, which are potentially supporting the electron emission in this setup: Firstly, the tip is heated by the driving laser. The influence of the resulting thermal blurring can be estimated by means of the electron gas temperature in the metal after the initial heating by the short laser pulse. Specifically, when assuming an intensity of about $10 \mathrm{GW} / \mathrm{cm}^{2}$ of the driving photoemission pulse (100 fs pulse duration, $30 \mu \mathrm{m}$ focal size, $300 \mathrm{kHz}$ repetition rate and $10 \mathrm{~mW}$ average laser power; corresponding to about $33 \mathrm{~nJ}$ pulse energy), we expect an initial temperature increase of the electron gas to about $1000 \mathrm{~K}$ for tungsten $[194,195]$. The FWHM of the derivative of the Fermi-Dirac distribution has a width of $0.3 \mathrm{eV}$ in this case, compared to $0.1 \mathrm{eV}$ at $T=300 \mathrm{~K}$. Hence, a thermally assisted two-photon process with a reduced effective nonlinearity can be expected [156, 196, 197].

Furthermore, the applied bias voltage at the tip leads to a decreased effective work function (also see Fig. 3.2C) following Eq. 3.1, likewise resulting in a decreased power law in Eq. 5.1 [186, 198]. This behavior can be easily verified by changing the suppressor voltage, which effectively controls the field enhancement at the tip apex (Sec. 4.2): we expect a decrease of the nonlinearity for higher fields (suppressor voltage closer to tip voltage) and a likewise increase of the nonlinearity for capacitor-like potential distributions (suppressor voltage close to cut-off value). Specifically, for a tip voltage of $-450 \mathrm{~V}$, the exponent in Eq. 5.1 is decreased from 1.88 to 1.61 when reducing the magnitude of the suppressor voltage from $-960 \mathrm{~V}$ to $-880 \mathrm{~V}$. The strong suppressor voltage dependence also serves as an indicator for electron emission from the apex. The reduced exponential in Eq. 5.1 is most likely a result from the combination of both effects.

For testing purposes, we operated the gun with up to about 100 electrons per pulse, but found this operation regime to be too close to the tip damage threshold at the employed high repetition rates $(312 \mathrm{kHz})$. In perspective of a stable long-term operation ${ }^{4}$, we therefore limited the maximum emission current to about 10 electrons per pulse. As will

\footnotetext{
${ }^{4}$ All sample measurements presented in the next chapter have been performed with the same tungsten tip without any noticeable degradation of the electron signal.
} 
be shown later, the resulting few million electrons on the detector per second allow for a real-time observation of diffraction patterns with reasonable contrast and necessitate long integration times only for high-accuracy measurements or low repetition rates [142].

A knowledge of the focusability and electronic current now also allows for the determination of the reduced brightness (see Eq. 3.9 and 3.11). Taking the solid angle of emission to be $\Omega=0.3 \mathrm{srad}$ [50], a current of $1 \mathrm{pA}$ (corresponds to $312 \mathrm{kHz}$ repetition rate and 20 electrons per pulse) and an typical electron energy of $300 \mathrm{eV}$, this results in a reduced brightness of $B_{r}=35 \mathrm{~A} / \mathrm{Vm}^{2} \mathrm{sr}$. This value is in the upper range of those reported for state of the art femtosecond photocathodes (see Ref. [182] for comparison). Taking into consideration the extremely small duty cycle of the electron source $\left(\approx 2.5 \cdot 10^{-8}\right)$, the resulting value is not far from the brightness achieved by static field emission sources [181].

The coherence of the electron source cannot be measured directly at this point. However, it is roughly estimated in (Sec. 6.2.2) from the angular width of the diffraction peaks.

\subsubsection{Temporal resolution of the ultrafast electron gun}

The temporal spread of the electron pulses at the sample position as well as their spatial and temporal overlap with the pump pulses is determined via a transient electric field (TEF) effect near a bare gold or copper TEM grid [148, 199-201]. The experimental principle is demonstrated in Fig. 5.7. First, the electron gun is operated with a divergent beam (zero lens voltage) to obtain a projection image of the TEM grid (Fig. 5.7B).

The pump beam is then approximately overlapped with the imaged region by observing the diffuse laser reflection on the grid at low fluence levels. Once the overlap is achieved, the pump fluence is increased again (greater than $10 \mathrm{~mJ} / \mathrm{cm}^{2}$ ). While changing the relative temporal delay $\Delta t$ between electron probe and laser pump via a translation stage (Fig. 5.4A), the projection image is recorded. For positive delay times (arrival of pump pulse before probe pulse), a distortion of the projection image can be observed (Fig. 5.7C and D). The distortion stems from the deflection of the electron trajectories of the passing pulse by Coulomb repulsion within the region of the electron cloud generated by the pump pulse. 

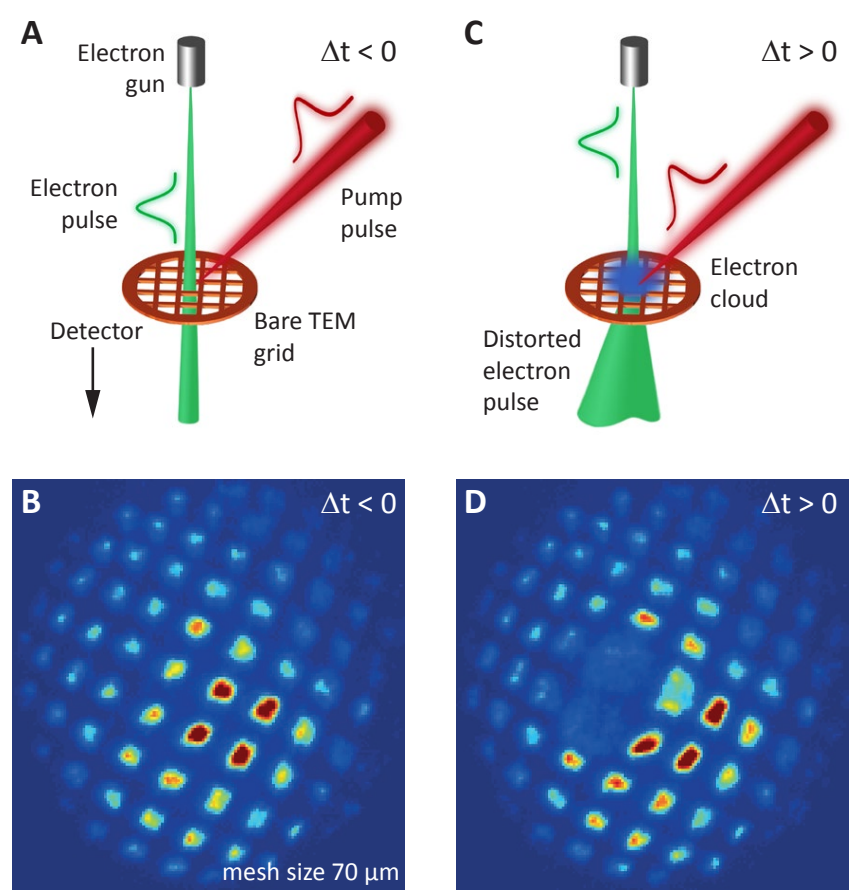

Figure 5.7: Pulse length determination via TEF effect. A: For negative delay times, the electron gun delivers an undisturbed projection image of a bare TEM grid (B). C: For positive delay times, the electron pulse propagation is altered by the pump-induced electron cloud on the TEM grid, resulting in a distorted projection image $(\mathbf{D})$. For a more detailed account on this technique, see Ref. [148, 199-201].

However, this distortion is only seen in part of the projection image and hence only applies to a partial beam. To obtain a measure for the pulse duration under normal operation conditions, e.g., in a diffraction experiment, the above measurement is repeated with the electron beam focused through a single mesh cell by adaption of the lens voltage. This technique requires a repositioning of the delay stage to maintain the temporal overlap.

The achievable temporal resolution of this method can be roughly estimated by the interaction time $t_{\text {int }}$ of the electron cloud emitted from the grid with the passing electrons [202]. When assuming the radial electron cloud diameter to be of the order of $r=100 \mu \mathrm{m}$ (as visible in Fig. 5.7D) and exhibiting a half-spherical shape on the TEM grid's surface [141], the interaction distance along the axis of propagation of the electrons is on average ${ }^{5}$ about $d_{\mathrm{int}}=\frac{2}{3} r$. This gives the following dependence between the interaction time and the electron energy $E$ for in the case of non-relativistic electrons

$$
t_{\text {int }}=d_{\text {int }} \sqrt{\frac{m_{e}}{2 E}}
$$

\footnotetext{
${ }^{5}$ With respect to the half-sphere's volume.
} 
where $m_{e}$ is the electron mass.

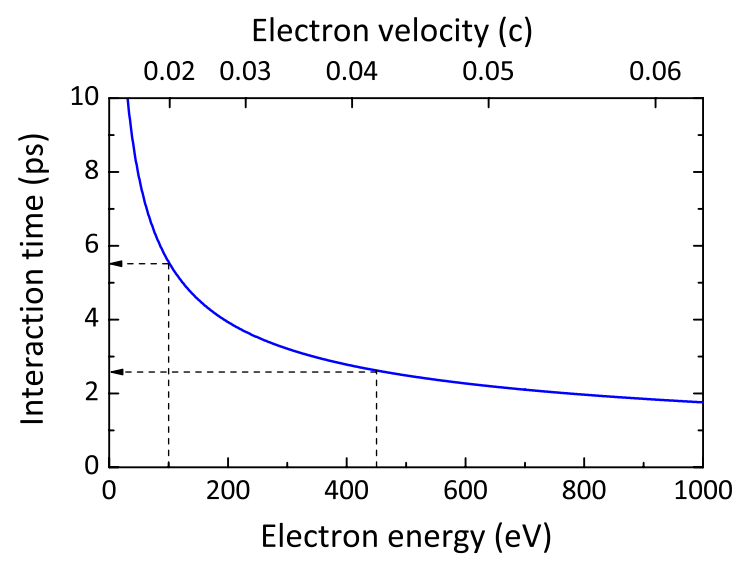

Figure 5.8: Estimate for the interaction time of a passing electron with energy $E$ through the electron cloud generated at the TEM grid surface. Dashed arrows indicate the interactions times for electrons of $100 \mathrm{eV}$ and $450 \mathrm{eV}$ to be about $5.5 \mathrm{ps}$ and $2.5 \mathrm{ps}$, respectively.

Figure 5.8 displays the estimated interaction time for energies between $1 \mathrm{eV}$ and $1000 \mathrm{eV}$, which is of about the same order of magnitude as the pulse durations computed in the simulations (Fig. 4.7) and in agreement with values found in the literature [148]. However, it also means that this method is likely to deliver only an upper limit for the pulse durations, especially for faster electron pulses (on the ULEED scale) of few hundreds of electron volts. In the light of this limitation, several alternative approaches to measure the pulse duration are discussed at the end of this chapter (Sec. 5.4.1).

The experimentally recorded, normalized intensity transients for two electron energies of a few-micron sized mesh region are displayed in Fig. 5.9. For a determination of the pulse duration from the recorded intensity evolution $I(t)$, we assume a static, instantaneously generated electron cloud at $t=t_{0}$ as well as a Gaussian temporal beam profile $g(t)$ of the electron cloud.

$$
\begin{aligned}
I(\Delta t) & =(\Theta * g)(\Delta t), \text { and } \\
D(\Delta t) & =D_{0}+D_{1} \operatorname{erf}\left(-\frac{2 \sqrt{2 \log (2)} \cdot I(\Delta t)}{\mathrm{FWHM}}\right),
\end{aligned}
$$

where $\Delta t=t-t_{0}, \Theta(t)$ is the Heaviside function, $D_{0}$ is an offset and $D_{1}$ is a normalization constant.

The recorded transients display a width of $7 \mathrm{ps}$ for $100 \mathrm{eV}$ and $2 \mathrm{ps}$ for $450 \mathrm{eV}$ electron energy. As expected, these values are very close to those estimated for the interaction 

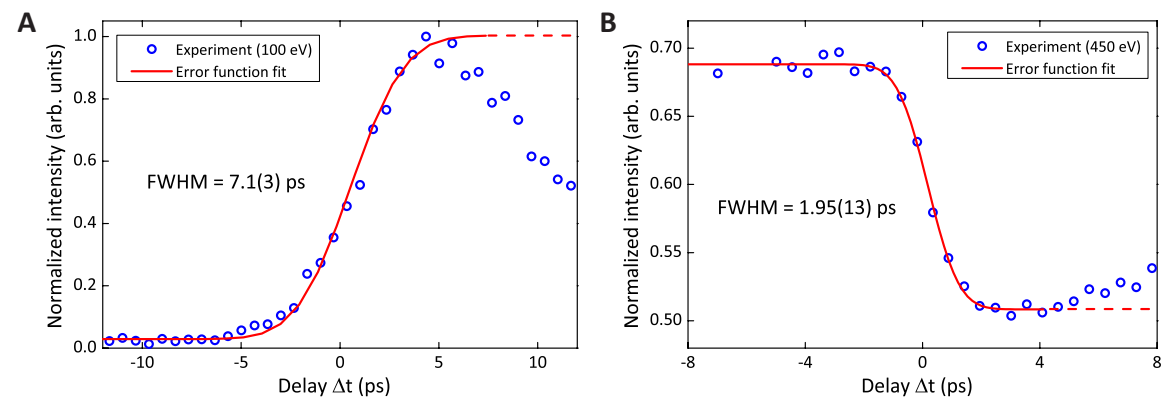

Figure 5.9: Pulse duration determination via TEF effect for different electron energies. Fluence: $20 \mathrm{~mJ} / \mathrm{cm}^{2}$. Electron energy: $100 \mathrm{eV}(\mathbf{A}), 450 \mathrm{eV}(\mathbf{B})$.

time with the plasma cloud, namely 5.5 ps and $2.5 \mathrm{ps}$, respectively (Fig. 5.8). Additionally, they do not take into account the formation time or propagation effects of the plasma cloud during the passing of the electron pulses. Therefore, these pulse durations are to be seen as an upper limit for a partial beam ${ }^{6}$. The actual pulse durations are therefore likely to be somewhere in between these values and those from the simulation.
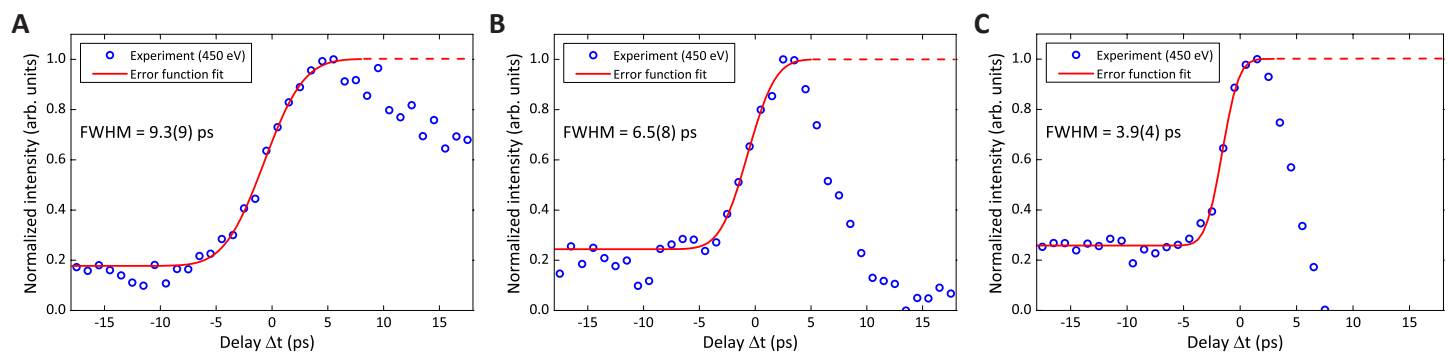

Figure 5.10: Fluence dependence of pulse duration measured via transient electric field effect evaluated at the same position. Pulse duration measured at same sample position for pump fluences of $2.3 \mathrm{~mJ} / \mathrm{cm}^{2}(\mathbf{A}), 4.2 \mathrm{~mJ} / \mathrm{cm}^{2}(\mathbf{B})$ and $10.4 \mathrm{~mJ} / \mathrm{cm}^{2}$ (C)

Notably, the measured temporal width of the intensity transient is a strong function of the pump fluence [203]. Figure 5.10 displays the above measurement (settings as in Fig. 5.9B) repeated for a set of lower fluences. A shortening of the recorded transients is somewhat counter intuitive, since one would expect an expansion of the plasma cloud at higher fluences to be accompanied by a growing interaction time with the passing electron pulse. This effect can be explained by the higher electron velocities in the emitted plasma cloud, which lead to a faster dissipation of the same [204]. Hence, for a more in-depth analysis of the achievable temporal resolution of this method, a model taking into account also the plasma dynamics would be better suited.

\footnotetext{
${ }^{6} \mathrm{An}$ integration over the whole mesh cell yields a longer pulse duration (about 6 ps in the case of $450 \mathrm{eV}$ electrons), caused by a significantly stronger impact of the plasma cloud formation and propagation time as well as by a spatial inhomogeneity of pulse durations within the electron beam.
} 


\subsection{Summary and discussion}

In this chapter, a gun design based on the previously introduced concept of a laserdriven needle emitter has been implemented and characterized. Specifically, a reduced nonlinearity compared to pure two-photon photoemission has been found, which could be attributed to a reduced effective work function by application of a bias voltage as well as heating of the photocathode by the driving laser. Furthermore, the energy spread was measured to be in the range of $1 \mathrm{eV}$.

The reduced brightness of the electron source was determined to be of the order of $B_{r}=35 \mathrm{~A} / \mathrm{Vm}^{2}$ sr at $300 \mathrm{eV}$ electron energy, which is comparable to flat pulsed photocathodes. The current setup features an energy-dependent temporal resolution between 2 ps $(450 \mathrm{eV})$ and 7 ps (100 eV) FWHM. All measured values compare well to the simulated ones, attesting for the further implementation of FEM computations in future source developments.

The main statement of this chapter is, however, that the developed electron source is well-suited for the time-resolved investigation of ultrafast dynamics at surfaces.

\subsubsection{Alternative approaches to determine the pulse duration}

The applied method of the TEF effect yields only a relatively rough estimate of the pulse duration [199]. For the employed prototype electron gun, this estimate may be sufficient. In light of further developments, however, experimental means for a more accurate determination of the temporal spread are needed. In the following, a few alternative approaches are discussed with respect to their ability to characterize lowenergy electron pulses in combination with an applicability in the current ULEED setup.

1. A temporal pulse characterization by ultrafast streaking [205-208]. The electron pulses are channeled through a narrow and rapidly changing electromagnetic field normal to their propagation direction. The detected electron position of the streaked pulses then depends on the relative arrival time of the electrons in the field region. From the length of the streaking pulses as well as the temporal evolution of the field, the pulse duration can be estimated. 
2. Similar to the streaking principle, the electron pulse duration can be determined via ponderomotive scattering by an intense laser pulse [209, 210]. Specifically, a short and intense pump pulse focused in the line of propagation of the electron pulse and polarized normal to it scatters passing electrons depending on their relative temporal delay.

3. Photon-induced near field electron microscopy (PINEM) [211]. The operation principle is based upon the observation that a temporal overlap of the electron pulse with an intense optical pulse in the close vicinity of a nanostructure leads to identifiable changes in the electron pulse spectrum.

4. A TEF effect on a second nanometric tip placed at the position of the grid.

5. The measurement of an ultrafast transient effect, which is significantly shorter than the electron pulse duration, thus effectively allowing for a sampling of the electron pulse length [212, 213].

The principle of ultrafast streaking is shown to work very well in the case of fast electron pulses for energies between $30 \mathrm{keV}$ [206] and several $\mathrm{MeV}$ [207] with temporal resolutions down to about $500 \mathrm{fs}$ and $200 \mathrm{fs}$, respectively. Similar to the case of the transient electric field effect on the copper grid, the quantity limiting the temporal resolution is the interaction time with the electric field. By relating the electron velocities and respective achieved temporal resolutions, the temporal resolution of a streaking setup in the present experiment can be estimated. Assuming $450 \mathrm{eV}$ electrons, this estimate yields a temporal resolution of about 4.3(0.6) ps (10 ps at $100 \mathrm{eV})$. Hence, a determination of the pulse duration by means of a streaking setup will only be meaningful after an improvement of the experimental setup, e.g., by further reducing the propagation distance through the capacitor.

Recently, so called laser streaking has been developed, which is based on the electron probe and laser pump beam being intersected at an ultrathin metal mirror. This concept potentially allows for sub-femtosecond temporal resolution, since it mainly depends on the duration of the employed laser pulses [208]. For high energy electron pulses, this method bears large potential for an extremely precise temporal characterization. Nevertheless, it has two distinct disadvantages in view of an application in ULEED: Firstly, phase-stabilized laser pulses are needed, and secondly, the metallic mirror must 
be thin enough for the electrons to pass. At the thickness needed to be transmissive for a sufficient percentage for low-energy electrons, such a mirror would most likely be mechanically and thermally unstable, when illuminated by intense, short laser pulses, hence making this approach challenging in the case of slow electrons.

Based on a similar concept, the temporal resolution achievable by ponderomotive scattering is also primarily determined by the optical pulse length and focal size employed. Hebeisen et al. [209] used 90 fs laser pulses and achieved a temporal resolution of about 100 fs. The major drawback of the method, however, is the requirement of very high pulse energies of several millijoules to achieve the necessary intensities of about $100 \mathrm{TW} / \mathrm{cm}^{2}$. However, for low-energy electrons, the necessary field strength is reduced, potentially allowing for an application of this method to ULEED. In streaking, the maximum energy $\Delta E$ gained from an optical field (wavelength $\lambda$, peak electric field $F_{\|}$along the electron's direction of propagation) can be approximated as [214]

$$
\Delta E=\frac{e \lambda}{\sqrt{2 m_{e}} \cdot \pi c} \sqrt{E_{0}} \cdot F_{\|}
$$

with $E_{0}$ the electron energy. In the experiment conducted by Hebeisen et al., $\Delta E$ was of the order of $E_{0}$. With our current laser system and experimental conditions (average pump power: $500 \mathrm{~mW}$, focal spot size: $20 \mu \mathrm{m}$, pulse duration: $80 \mathrm{fs}$, repetition rate: $312 \mathrm{kHz}$, wavelength $800 \mathrm{~nm}$ ), we would only achieve about $15 \%$ of the needed energy gain at an electron energy of $100 \mathrm{eV}$. However, when using a microscope objective for focusing, this approach could potentially be made feasible in a ULEED setup.

PINEM employs strong near fields in the vicinity of laser-illuminated nanostructures to (de-) accelerate passing electrons [211]. The interaction time between the nanostructure and a passing electron pulse is, due to the small nanostructure diameter, only in the range of few femtoseconds, even for relatively slow electrons $(\approx 17$ fs for $100 \mathrm{eV}$ electrons and a $100 \mathrm{~nm}$ thick nanowire). Due to the field enhancement effect at the nanostructure (Sec. 3.3.3), the laser pulse energies of few microjoule used are comparable to those for sample excitation in most ultrafast experiments. However, the experimental effort especially with respect of the measurement of the electronic spectra and the large beam diameter of the ULEED setup, is relatively high.

Yet, a partial application of the concept introduced in PINEM would potentially lead to a drastically increase of the temporal resolution of the current TEF effect-based method: 
Specifically, the influence of a TEF effect on the electron pulse is recorded at a second sharp needle emitter (instead of the copper grid). The second needle is positioned to have its symmetry axis perpendicular to the electron's direction of propagation and is likewise illuminated by the pump laser. By optical field enhancement, electrons around the tip apex are strongly scattered. To allow for an even stronger enhancement caused by resonant plasmonic excitation at the surface, taking into account the $800 \mathrm{~nm}$ driving pulses in our current setup, a gold instead of a tungsten tip could be employed.

The big advantage of this method would be the relatively modest engineering effort to implement a second tip and a potential temporal resolution of about $100 \mathrm{fs}$, in principle limited only by the driving laser of the second tip. However, the extremely small interaction area compared to the large beam diameter of the electron pulse results in a small scattering efficiency, thus necessitating long integration times to observe a transient behavior. Specifically, taking the ULEED electron pulse cross section to be $A_{\text {pulse }}=\pi(30 \mu \mathrm{m})^{2}$ and the interacting tip surface to be of the order of $A_{\text {int }}=\pi(10 \mathrm{~nm})^{2}$, the scattering efficiency is about $10^{-7}$. This would mean that even at high beam currents, only one scattered electron every two seconds is detected. For a rough estimate of the necessary integration time, the following parameters are taken: The employed $\mathrm{MCP}^{7}$ has an effective diameter of $77 \mathrm{~mm}$, a spatial resolution of about $1 \mathrm{~mm}$ and a dark count of the order of 10 electrons per second at single-electron sensitivity. Hence, when assuming that the non-scattered electrons can be efficiently blocked without losing the scattered electrons, an integration time of few tens of seconds should be sufficient for a discernible signal (approx. $30 \mathrm{~dB}$ signal to noise). However, this estimate is rather optimistic and does not take into account additional noise producing effects, including electronic noise from the camera's charge-coupled device (CCD) or electrons occasionally scattered from lens assembly apertures.

A similar straight-forward approach as the one just discussed would be to determine the pulse duration via an ultrafast structural effect, such as a reduction of the diffraction intensity due to lattice heating (about $\tau \approx 200$ fs for graphene / graphite, [213], but potentially masked by transient electric field effects [204]) or the structural change observed in vanadium dioxide $\left(\mathrm{VO}_{2}\right)$ upon transition from insulator to metal (in particular the $\mathrm{V}-\mathrm{V}$ bond dilution, $\tau \approx 300 \mathrm{fs}$, [35]). The major advantage of this approach would be

\footnotetext{
${ }^{7}$ Hamamatsu F2226-24p, data sheet available at: http://www.datasheetarchive.com/hamamatsu\%20F2226-datasheet.html
} 
the applicability in a transmission (thin film dynamics) as well as in a reflection (surface dynamics) geometry.

\subsubsection{Further improvements on the gun design}

As has been demonstrated in the simulations, the achievable pulse duration is basically limited by two major factors, namely the propagation distance to the sample and the energetic spread of emitted electrons. In the following, approaches to further decrease the temporal spread based on these two factors, are discussed.

The pulse duration increases roughly linearly with the propagation length, as can be seen in Eq. 3.13. A reduction of the pulse duration by means of a miniaturizing of the electron gun in general is therefore a relatively straight-forward approach. In fact, an electron gun currently being developed in our laboratory employs apertures from electron microscopy to limit the total propagation distance to few millimeters, thus potentially allowing for sub-picosecond resolution. Additionally, the gun diameter is strongly decreased, which facilitates the recording of diffraction images in the classical back-reflection LEED geometry (see Sec. 7.2).

An alternative approach is the lensless diffraction by a nanometric needle emitter directly in front of the sample [215]. The approach has shown to allow for about 250 fs temporal resolution at electron energies as low as $70 \mathrm{eV}$ with excellent focusability. However, there are still particular challenges of this geometry due to the vicinity of the emitter to the sample, including the influence of the pump pulse on electron emission, the necessary shielding of back-reflected electrons from the electrostatic tip and suppressor potentials, as well as the transfer of this approach to a backscattering geometry without largely shadowing the sample.

Ultimately, a nanofabricated electron lens system would be ideal, offering small source dimensions, hence minimizing propagational broadening and shadowing effects, while allowing for full electron beam control.

The second strong influence on the pulse duration is velocity dispersion, originating from a finite initial energy distribution width of the emitted electrons. This width has several contributions, including thermal blurring of the Fermi-Dirac distribution of the electron 
gas in the metal, different electron momenta within the metal surface, finite width of the spectrum of the driving laser, and the non-planar emission geometry of the tip itself.

A first step in reducing the energy spread is the use of tungsten with a single crystal orientation for the production of the tips, since, depending on the surface direction, tungsten has work functions between $4.5 \mathrm{eV}$ (111) and $5.3 \mathrm{eV}$ (110) [192]. Without this initial step, the efficiency of the subsequently explored methods would be strongly reduced.

The influence of the thermal blurring has previously been estimated by means of the electron gas temperature in the metal after the initial heating by the short laser pulse (Sec. 5.3.1). Specifically, it was found that for tungsten, the FWHM of the derivative of the Fermi-Dirac distribution is $0.3 \mathrm{eV}$ under typical experimental conditions as found in ULEED, compared to $0.1 \mathrm{eV}$ at $T=300 \mathrm{~K}$. The enhanced tip temperature leads to thermally assisted photoemission, which results in a broader energy distribution of the emitted electrons. To minimize this effect, the photocathode can be cooled [216].

Additionally, the wavelength of the photoemission laser can be adapted to avoid large excess kinetic energies of electrons after the emission process. This could be achieved by means of an optical parametric amplifier (OPA). A disadvantage of a carefully aligned wavelength would be that whenever the potentials of the suppressor, tip or extractor unit are changed, the wavelength would also have to be realigned to compensate for the modified effective work function, see Eq. 3.1. In the typical operation regime of the current ULEED setup, the change in work function is of the order of $0.2 \mathrm{eV}$ when increasing the electron energies of $300 \mathrm{eV}$ to $450 \mathrm{eV}$. However, by adapting the electric potentials of all three components in such a way that the field around the tip is not drastically altered, this change can be minimized.

A reduction of the initial kinetic energy of the electrons would also reduce the energy spread caused by the tip geometry. The spread is caused by emission of electrons with velocity components normal to the forward propagation direction. Specifically, an electron with initial energy $E_{0}$ emitted under an angle of emission $\beta$ relative to the surface normal at the emission site $\alpha$ (Sec. 4.1.1) has an energy component parallel to the forward direction which is reduced by

$$
\Delta E=E_{0}(1-\cos (\alpha+\beta)) .
$$


This difference in kinetic energy is somehow damped by the trajectories being bent in forward direction, depending on the lens assembly voltage settings. A reduction of the initial energy $E_{0}$ would likewise decrease the contribution of the emission geometry to the overall energy spread.

Specific efforts in the direction of the development of an improved electron source are presented in the outlook of this work (Sec. 7.2).

\subsubsection{Optical field emission electron gun}

As described in this section, the pulsed electron gun in this setup is operated in the multiphoton regime (see Fig. 5.6B). An operation at even higher intensities of the driving laser is theoretically possible, and could even lead to shorter electron pulses with higher spatial coherence.

In the regime of optical field emission (Sec. 3.3.3), the driving light field is dominating over the electronic binding forces or the metal, thus basically ripping the electrons out of the tip. After emission, electrons could potentially gain a significant amount of kinetic energy from the strongly enhanced oscillating light field in the vicinity of the nanostructure [49], thereby also reducing the solid angle of emission [175]. The energy gain would depend strongly on the phase of the laser pulse at the time of emission and can easily be in the region of hundreds of electron volts for long enough wavelengths. However, this high energy gain has to be paid for by a broad electron energy spectrum, resulting from its strong phase-dependency [217].

Nevertheless, with accurately timed, phase-locked laser pulses and appropriate spectral compression methods, e.g., by RF-cavities [138, 148, 218] an optical field emission electron gun could in principle lead to enhanced temporal resolution at low electron energies. A main drawback of such an electron source would be the limited repetition rate within a pump / probe scheme. To avoid strong cumulative heating effects resulting in a thermal destruction of the metal tip, the operation would be likely limited to repetition rates of the order of few $\mathrm{kHz}$, resulting in long integration times due to the relatively small electron-per-pulse yield of needle-type emitters [50]. Additionally, the temporal compression of the electron pulses is paid for by a broadened energy spectrum. Considering, for example, the relatively large RF-cavities in combination with the strong 
propagational broadening at low electron energies, the realization of such a scheme will likely be technically challenging.

From the point of view of this work, the operation of such a laser driven optical field emission electron gun is in principle feasible, but will require a rather sophisticated experimental setup compared to the one at hand.

\subsubsection{THz compression of electron pulses}

Very recently, optical temporal compression of electron pulses photoemitted from a nanometric tip has been demonstrated experimentally in our group [217]. In particular, a pulsed, needle-type photocathode has been illuminated by an additional $\mathrm{THz}$ pulse, effectively leading to a streaking and gating of the photoelectron emission by modulation of the metal work function. Depending on the relative temporal delay between the photoemission and the $\mathrm{THz}$ pulses, the energetic electron spectra have been compressed, shifted or broadened.

This approach, which can be understood as the optical equivalent of RF compression, offers two distinctive advantages compared to the cavity-based approach: firstly, the THz-based technique operates at orders of magnitude higher frequencies, offering few-fs temporal control [219]. Secondly, the electron pulses are manipulated directly at the emission site, therefore avoiding a temporal broadening caused by longer propagation distances, allowing for a potential implementation of the technique in ULEED. 


\section{Chapter 6}

\section{Ultrafast PMMA superstructure dynamics on free-standing graphene}

This chapter describes the time-resolved structural analysis of an ultrathin polymer overlayer adsorbed on free-standing graphene by the previously introduced transmission ULEED setup. Part of the results presented here have been published in Ref. [220].

After a short motivation of the selected sample system (Sec. 6.1) and a brief description of the sample preparation method (Sec. 6.1.1), ULEED is used to perform an initial characterization of the graphene / PMMA bilayer system (Sec. 6.2). Further, the nonequilibrium dynamics of a crystalline PMMA superstructure are analyzed and the time scales of different involved processes identified (Sec. 6.3). Lastly, we propose a physical picture of the superstructure evolution (Sec. 6.4), which is subsequently discussed (Sec. 6.5).

\subsection{Choice of a sample system}

The previous chapters have theoretically as well as experimentally demonstrated the potential performance of ULEED based on its brightness, its spatial and its temporal resolution. However, the determination of all the key quantities has been performed in 
isolation from each other. Therefore, it is important to additionally present a feasibility study, based on the analysis of a complex sample system, to demonstrate the applicability of ULEED as a novel tool for ultrafast surface science.

We therefore prepare a sample system consisting of an ultrathin polymer layer on freestanding graphene. From a fundamental point of view, this system is interesting, because its constituents display drastically different mechanical, electronic and thermodynamic properties. In particular, whereas the polymer, atactic PMMA, usually does not exist in crystalline form, graphene is a highly-ordered system. Moreover, while individual PMMA chains with molecular weights of close to $10^{6} \mathrm{~g} / \mathrm{mol}$ are expected to react rather slowly on environmental changes [221], graphene's relaxation dynamics have been shown to happen on very short time scales $[203,222]$.

On the other hand, the properties of these two materials are often interlaced in terms of graphene heterostructures [18], and, even more strongly, graphene / polymer composite materials [79]. But also apart from graphene-based material compositions, PMMA is commonly incorporated into the preparation process of free-standing graphene [103], and to our knowledge also regularly seen on TEM images [103, 105, 223]. However, concerning the interplay between these two materials, there is still a pronounced lack of understanding. One reason might be that even though the graphene subsystem and its dynamical properties are routinely mapped [203], the dynamical investigation of the PMMA layer is challenging. Major difficulties in the investigation include PMMA's transparency over a wide spectral range [224] as well as its high susceptibility to electron beam induced damage [225].

In light of a fundamental interest as well as its importance in graphene research and technology, a PMMA / graphene bilayer represents a potentially very rich system to study. Furthermore, the extremely low radiation-dose of ULEED compared to other time-resolved electron diffraction experiments might allow for a new perspective on the dynamics of graphene composites relaxing from highly non-equilibrium states, which have been inherently difficult to access so far. 


\subsubsection{Sample preparation}

Graphene was first obtained by means of the scotch-tape method as described in [71]. Since then, many different preparation methods have emerged ultimately trying to allow for a high-quality, low-cost, large-area graphene production [226]. An overview on alternative preparation methods can be found in Ref. [227] with focus on chemical vapor deposition (CVD).

The sample preparation process as used in the framework of this thesis is sketched in Fig. 6.1.

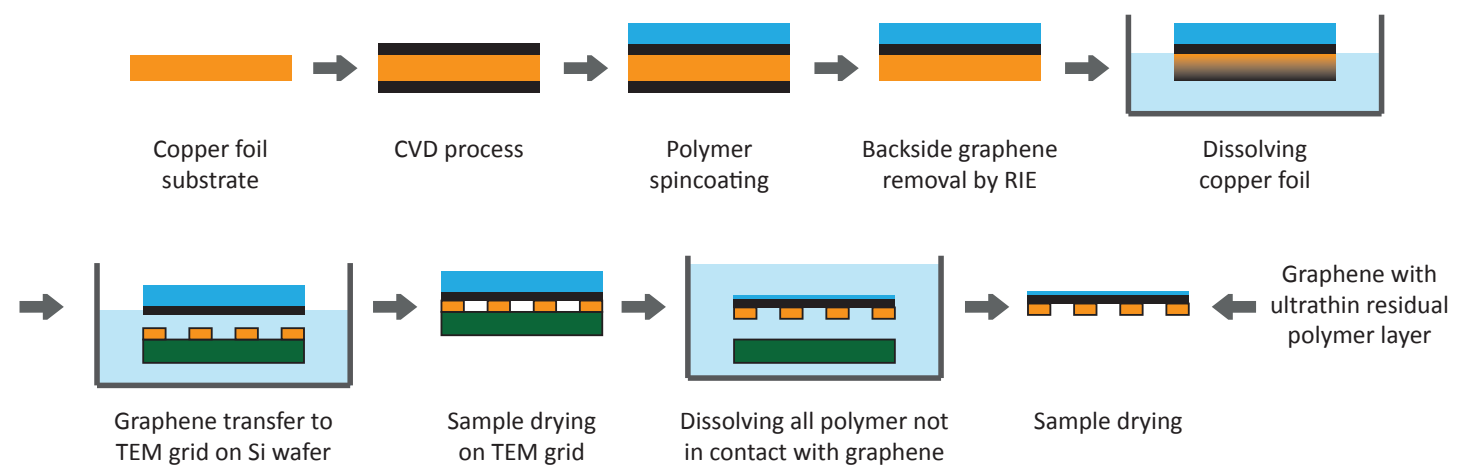

FigURE 6.1: Sample preparation of polymer/graphene bilayer system. Production of monolayer graphene and subsequent polymer-supported transfer to TEM grid.

Specifically, monolayer graphene is produced by a CVD process on a $25 \mu \mathrm{m}$ thick, preetched $\mathrm{Cu}$ substrate ${ }^{1}$. The following steps are undertaken within the CVD process $([103,220])$ :

1. First, the quartz tube reaction chamber is evacuated to a pressure of about $3 \mathrm{mTorr}^{2}$ by a mechanical pump.

2. Then, a $40 \mathrm{sccm}$ hydrogen gas flow is introduced at a pressure of $950 \mathrm{mTorr}$.

3. To enlarge the $\mathrm{Cu}$ grains and remove residual $\mathrm{Cu}$ oxide and impurities from the foil's surface, the $\mathrm{Cu}$ is heated to $1000{ }^{\circ} \mathrm{C}$ within $60 \mathrm{~min}$ and subsequently annealed for $30 \mathrm{~min}$.

\footnotetext{
${ }^{1}$ Vendor: Alfa Aesar, item No. 13382, purity: $99.8 \%$.

${ }^{2}$ The pressure scale of the instrument is in Torr. Torr are converted into Pa by multiplication with $101325 / 760$.
} 
4. For the graphene synthesis, methane gas together with hydrogen is introduced into the chamber (6 sccm and $20 \mathrm{sccm}$, respectively) for $10 \mathrm{~min}$ at a total pressure of 460 mTorr.

5. Lastly, the furnace is rapidly cooled down to room temperature, keeping the 20 sccm hydrogen flow.

In a next step, free-standing graphene is obtained by a transfer of the graphene to a TEM grid. To suspend the graphene and secure its adhesion to the grid, it is covered by a thin, holey layer of amorphous carbon (Quantifoil ${ }^{3}$ ), as visible in Fig. 6.2. For the sample used in this work, the hole size of the Quantifoil is either $3.5 \mu \mathrm{m}$ or $7 \mu \mathrm{m}$. At larger sizes the adhesion of the graphene to the substrate is decreasing rapidly.
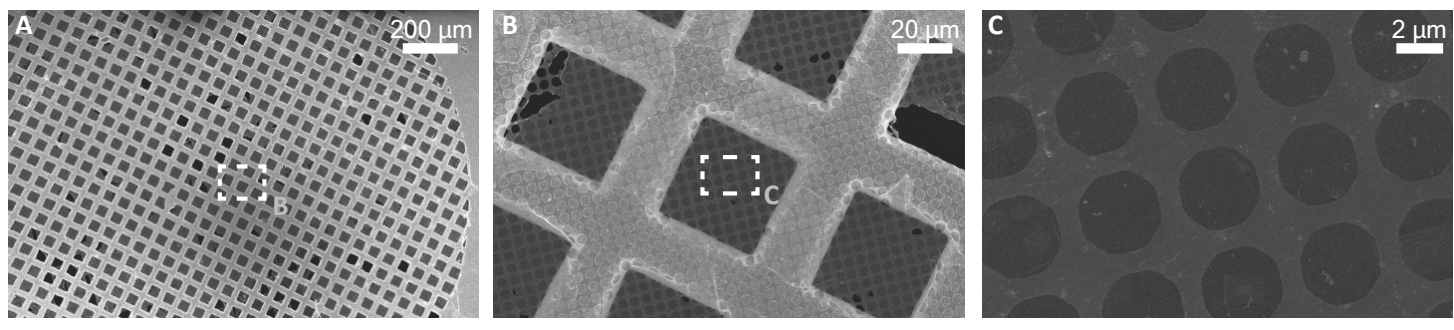

FiguRE 6.2: TEM grid covered with a thin layer (about $20 \mathrm{~nm}$ ) of amorphous, holey carbon (Quantifoil). A, B, and $\mathbf{C}$ show different magnifications of the TEM grid on the covering Quantifoil film. The dashed white rectangles show the areas magnified in the image to the right.

For the transfer process (Fig. 6.1), one side of the graphene / $\mathrm{Cu}$ foil is spincoated with a polymer. In this work, various types of polymers for spincoating have been used, namely two different solutions of $\mathrm{PMMA}^{4}$ and a single solution of $\mathrm{PC}^{5}$. The spincoating is performed at $2000 \mathrm{rpm}$ for $60 \mathrm{~s}$, the sample is subsequently dried for $1 \mathrm{~h}$ under atmospheric conditions. Backside graphene on the $\mathrm{Cu}$ substrate is then removed by oxygen plasma etching $(30 \mathrm{~s}$ at $100 \mathrm{~W})$. The remaining copper is dissolved in a $0.3 \mathrm{M}$ $\left(\mathrm{NH}_{4}\right)_{2} \mathrm{~S}_{2} \mathrm{O}_{8}$ solution for $6 \mathrm{~h}$. Finally, the left over polymer / graphene film is scooped out of the solution by TEM-grids ${ }^{6}$, which have been attached to a $\mathrm{Si}$ wafer by a small

\footnotetext{
${ }^{3}$ More information on Quantifoil on: http://www.quantifoil.com/.

${ }^{4}$ Named $\mathrm{PMMA}_{i}$ and $\mathrm{PMMA}_{i i}$; vendor: Allresist (http://www.allresist.de/); tacticity: atactic; molecular weight: $950,000 \mathrm{~g} / \mathrm{mol}$; PDI: 3.76 ; melting temperature: $130{ }^{\circ} \mathrm{C}-160{ }^{\circ} \mathrm{C}$; glass transition temperature: $105^{\circ} \mathrm{C}$; viscosity at $25^{\circ} \mathrm{C}$ : $\mathrm{PMMA}_{i} 43.4 \mathrm{mPas}, \mathrm{PMMA}_{i i} 8.8$ mPas; solvent: $\mathrm{PMMA}_{i}$ solved in anisole, $\mathrm{PMMA}_{i i}$ solved in ethyl lactate. Since most of the measurements and especially all time-resolved experiments are performed with samples spincoated with $\mathrm{PMMA}_{i}$, it is referred to as simply "PMMA", whereas the use of PC and PMMA ${ }_{i i}$ is specifically indicated.

${ }^{5}$ Poly(propylene carbonate); vendor: Sigma-Aldrich (http://www.sigmaaldrich.com/); tacticity: atactic; molecular weight: $50,000 \mathrm{~g} / \mathrm{mol}$; glass temperature: $25{ }^{\circ} \mathrm{C}-45{ }^{\circ} \mathrm{C}$; molecular weight: $102 \mathrm{~g} / \mathrm{mol}$; solvent: ethyl acetate.

${ }^{6}$ If not denoted otherwise, 400 mesh copper grid covered with a Quantifoil film of about $10 \mathrm{~nm}$ thickness and hole size of $3.5 \mu \mathrm{m}$. Vendor: Plano (http://www.plano-em.de/).
} 
amount of the same polymer as used during spincoating.

To increase the adhesion of the graphene on the grid, the samples are dried for several days under atmospheric conditions at room temperature. After this period, all polymer but the one in direct contact with the graphene substrate is removed by consecutively washing the sample for 15 min each in acetone, isopropanol, and DI water in the case of PMMA and chloroform, isopropanol, and DI water in the case of PC.

This fabrication protocol applies to all of the samples investigated in this work. A more detailed account on the graphene characterization, the influence of the drying time on the bilayer, as well as its thermal stability and degradation behavior under electron irradiation is provided in App. B.

\subsection{Bilayer characterization in the ULEED setup}

\subsubsection{Local diffractive probing}

For a first sample overview, the setup is operated in projection mode ${ }^{7}$ with a strongly divergent beam (Fig. 6.3A). The dark areas on the TEM grid denote sample coverage, whereas the few visible very bright mesh cells in the bottom right area of Fig. 6.3A indicates missing or ripped Quantifoil ${ }^{8}$.

In order to demonstrate bilayer abundance, local diffractive probing on a TEM grid with relative poor overall sample coverage has been performed. Fig. 6.3B shows a magnification of the area in the red dashed rectangle of Fig. 6.3A. Diffraction images have then been taken from each single mesh cell of the $3 \times 3$ mesh array in Fig. 6.3C. The green boxes mark mesh cells with notable diffraction signal, clearly showing that the graphene / PMMA bilayer covers only the dark areas within the projection images. An in-depth analysis of the recorded diffraction pattern is performed in the next section.

The dark spot just below the central beam block in (Fig. 6.3C) is visible in all diffraction images and originates from the geometry of the MCP detector. In particular, the

\footnotetext{
${ }^{7}$ Suppressor voltage optimized for homogeneous illumination of the whole sample area, lens voltage set to ground.

${ }^{8}$ Mean-free path length for $500 \mathrm{eV}$ electrons is of the order of $1 \mathrm{~nm}$, whereas the Quantifoil thickness is about $20 \mathrm{~nm}$, therefore not transmissive. Quantifoil coverage is about $50 \%$ on an intact sample site. More information on this subject in App. B.
} 

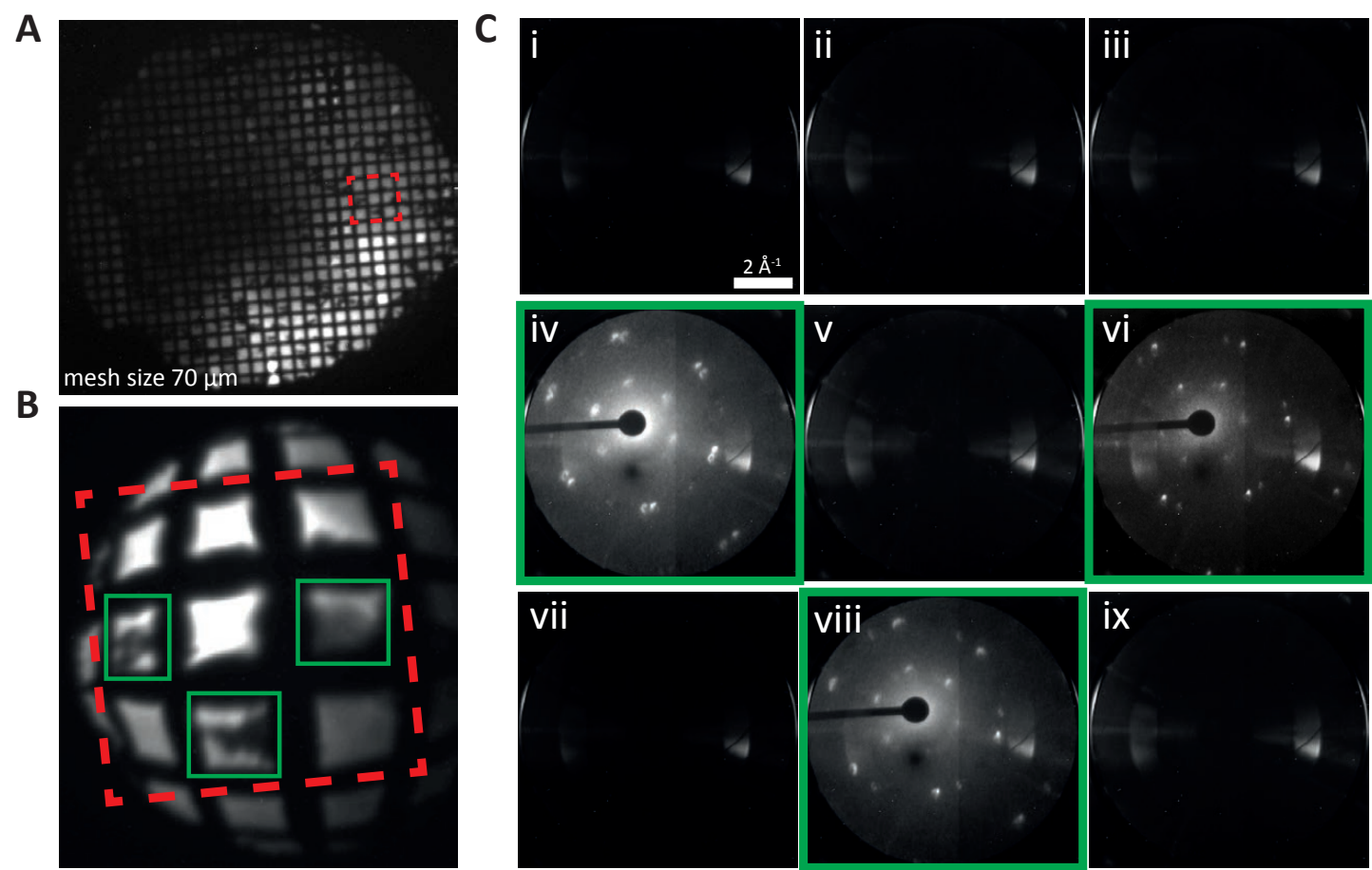

ix

Figure 6.3: Projection and local diffractive probing images of sample system. A: Projection image of sample, diffractive probing area is indicated by red dashed rectangle. B: Magnified area of dashed rectangle depicted in A. Green squares in B mark sample sites with notable diffraction signal. C: Diffraction images from individual mesh cells as indicated in B. Electron energy: $450 \mathrm{eV}$.

microchannels inside the MCP are tilted by $8^{\circ}$ with respect to the surface normal. At the spot position, electrons are propagating towards the screen at the same angle and are therefore subject to a reduced probability of amplification ${ }^{9}$.

In the framework of this thesis, the operation conditions have been chosen to be optimal for monolayer sensitivity, short pulse durations and high scattering efficiency. This resulted in electron energies above those typically employed in static LEED experiments.

However, is has to be stressed that the chosen energies are motivated only by the observed sample system and not a result of limitations of the electron source. In fact, the gun has been operated at electron energies below $100 \mathrm{eV}$, not showing any reduction of the beam quality.

Since the present setup is working in transmission, electrons need sufficient energy to be transmitted through the bilayer. This property can be observed in the dramatic increase of integration time, when reducing the electron energy below about $240 \mathrm{eV}$,

\footnotetext{
${ }^{9}$ We gratefully acknowledge discussions with M. Müller, which contributed greatly in understanding this effect.
} 
corresponding to a penetration depth of about $6 \AA$ (App. A.2). For comparison, the integration time for the diffraction image recorded in Fig. 6.3C at an energy of $450 \mathrm{eV}$ is only $10 \mathrm{~s}$, while the images displayed in Fig. 6.4, at an energy of $150 \mathrm{eV}$, are integrated over $200 \mathrm{~s}$ and those in Fig. 6.5 for only one second at an energy of $1000 \mathrm{eV}$.
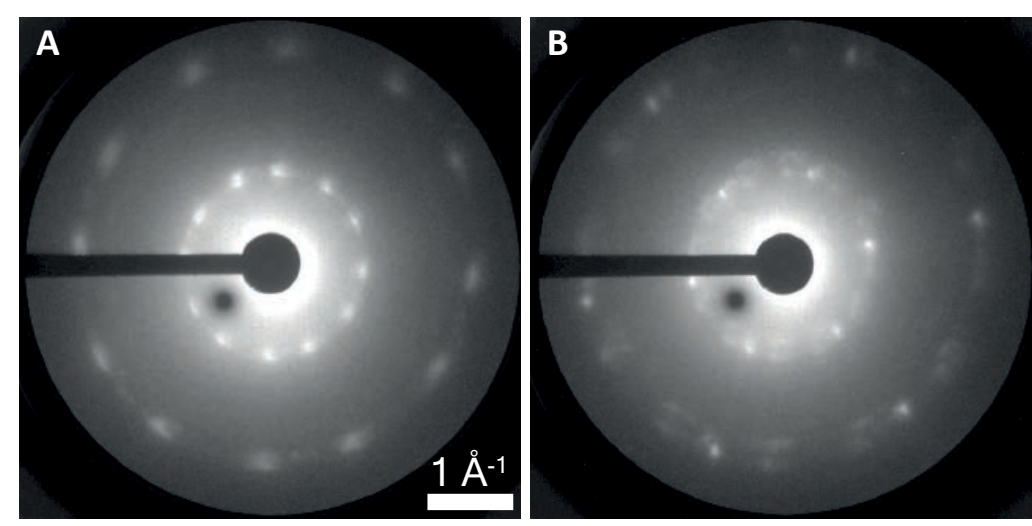

Figure 6.4: Diffraction images taken at $150 \mathrm{eV}$ at two different sample positions. Integration time: $200 \mathrm{~s}$. Non-linear gray scale for better visibility.

\subsubsection{Structural analysis of the polymer overlayer}

The deterioration of the sample by intense laser irradiation as well as electron bombardment have made it necessary to frequently change sample sites after long measurement runs. Therefore, typically samples with higher overall coverage were chosen, as displayed in Fig. 6.5A (coverage about $40 \%$ ).
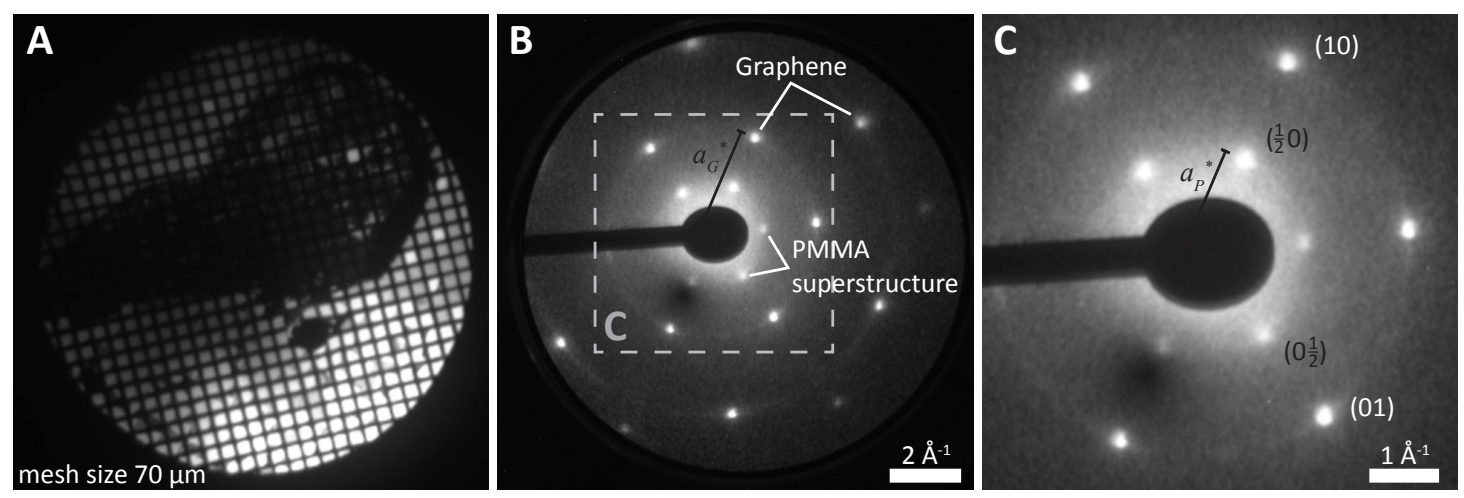

Figure 6.5: Projection and diffraction images of the PMMA/graphene bilayer system. A: Projection image from a sample recorded with the ULEED electron gun and divergent beam settings. Dark areas denote sample coverage. Electron energy: $450 \mathrm{eV}$. B: Diffraction image of a single mesh cell. The sample displays the typical hexagonally symmetric graphene peaks as well as an orientationally linked PMMA superstructure close to the central beam stop. Reciprocal graphene lattice parameter $a_{G}^{*}=2.95 \AA^{-1}$. Integration time: $1 \mathrm{~s}$, electron energy: $1000 \mathrm{eV}$. Dashed rectangle indicates area magnified in C. C: Close-up of graphene and PMMA superstructure diffraction peaks with Miller indices. Superstructure lattice parameter $a_{P}^{*}=1.47 \AA^{-1}$. 
On such a high-quality sample, a detailed diffraction image can usually be obtained within a few seconds, depending on the electron energy employed. Fig. 6.5B displays a typical diffraction pattern from a single mesh cell with full graphene / PMMA coverage. In particular, the hexagonally symmetric graphene diffraction spots with a reciprocal lattice parameter of $a_{G}^{*}=2.95 \AA^{-1}$ as well as an equal number of additional peaks closer to the central beam stop can be observed. These peaks are found to be at half the scattering angles of the graphene spots, corresponding to a lattice parameter of $a_{P}^{*}=1.47 \AA^{-1}$ (Fig. 6.5C). Moreover, they also display a six-fold symmetry and can be attributed to a superstructure formed by the ultrathin PMMA film. As seen in the diffraction pattern, this superstructure is orientationally linked to the graphene lattice.

Very similar superstructures are found on all samples prepared under comparable fabrication protocols (Sample preparation), using one out of two different polymers, namely PMMA and PC. Figure 6.6 displays two diffraction patterns found in the case of a PC / graphene bilayer system.
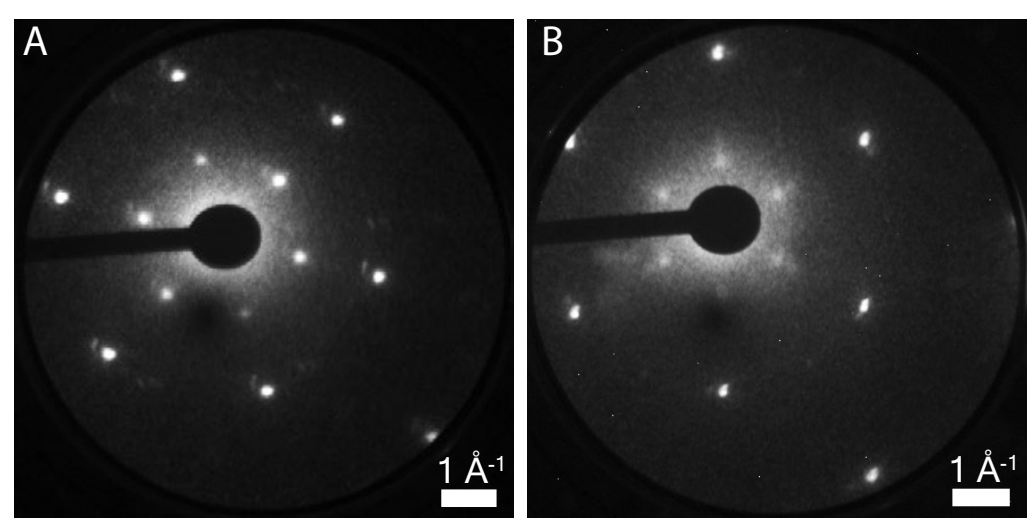

FiguRE 6.6: Superstructure types of PC/graphene bilayer system with different lattice parameter. $5 \mathrm{~s}$ integration time.

Orientationally, they display the same angular linkage to the graphene substrate, but differ significantly in intensity and lattice parameter. Whereas the reciprocal superstructure lattice constant in Fig. 6.6A is half that of graphene, the one found in Fig. 6.6B is about $30 \%$ smaller, displaying a reciprocal lattice constant of $a_{P C}^{*}=1.16 \AA^{-1}$. For a physical explanation of this effect, the impact of the monomer characteristics on the conformation of the superstructure has to be further investigated. Current efforts in this direction are pointed out in the outlook section of this work (Sec. 7.2).

Hence, in the time-resolved study, only graphene / PMMA samples with regions displaying a hexagonal pattern are chosen for the quantitative analysis of the diffraction 
images. Such samples have the advantage of usually featuring the best signal-to-noise ratio. Moreover, due to the limited resolution of the detector camera, intensity blurring between adjacent spots can be avoided. However, only about $20 \%$ of the probed sample areas display this symmetry, whereas the rest have shown a superposition of several different sample orientations. Most abundant are the two types of 12-fold symmetry depicted in Fig. 6.7A and B. Specifically, the individual patterns display relative angles of rotations of $\alpha=28^{\circ}$ and $\beta=21^{\circ}$, respectively. These tilt angles agree well with the theoretically predicted and experimentally confirmed most stable configurations of armchair- and zigzag-type grain boundaries of graphene (Sec. 2.3.1), which have been found to be $28.7^{\circ}$ and $21.7^{\circ}$, respectively [89-91].
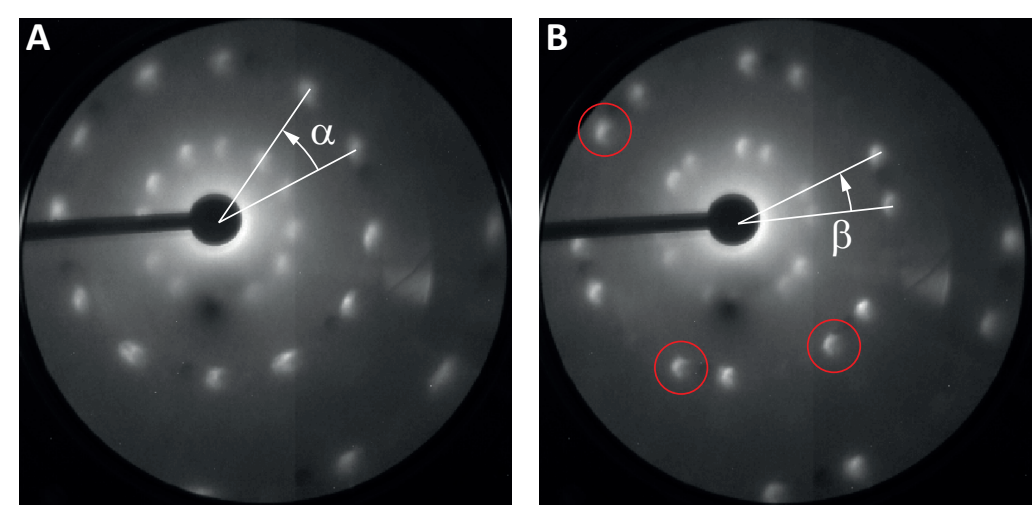

FIGURE 6.7: Diffraction images of typical sample regions featuring two distinct graphene orientations. Relative angular orientation of $\alpha=28^{\circ}(\mathbf{A})$ and $\beta=21^{\circ}$ (B). Red circles indicate clearly visible diffraction spot structures.

Additionally, the diffraction spots display a structure in terms of intensity distribution as marked by the red circles in Fig. 6.7B. Such an inhomogeneous intensity profile could in principle be an indicator of defects within the crystal lattice causing tension or mosaic formation and be interpreted as a result of the observed grain boundaries [69].

However, the observed relative angles between different graphene orientations are found to induce relatively little strain to the carbon bonds [91]. Moreover, a structured spot profile is also visible in the case of sample regions displaying a six-fold symmetry, which would be expected to have fewer defects compared to those depicted in Fig. 6.7. A more likely explanation is therefore an inhomogeneity not within the crystal lattice of graphene, but instead in the beam profile of the electron source. Particularly, the spot profile shows the same half-moon like shape as the intensity distribution of the electron beam, when operated in projection mode. This is likely caused by a combination of a slightly misaligned tip, an imperfect lens system, but also the tip geometry itself [228]. 
Generally, a measurement of transverse coherence in the framework of an electron diffraction experiment is relatively laborious. Nevertheless, the distance of the spots to the zeroth order spot (at the center of the pattern) with respect to their spatial extension can serve as a rough estimate. In particular, the ratio of peak width to peak distance to the pattern center is found to be $5-10$ is most cases, corresponding to a coherently illuminated sample area of about $2.5-5 \mathrm{~nm}$ in diameter. If compared to the value predicted for the maximal transverse coherence length (see Sec. 3.4.3 and Sec. 3.4.1), this value is orders of magnitude smaller. Possible reasons for the apparent reduction of the coherence length again include imperfect beam optics and a widening of the spot profile due to heating and superstructure degradation (see App. B.4). The latter can be excluded by using the graphene spots as a scale instead, resulting at similar values for the transverse correlation length. Additionally, as will be demonstrated in the outlook (Sec. 7.2), a newly designed miniature electron gun produces significantly sharper diffraction peaks, supporting the idea of a limitation of the coherence length by imperfect electron optics in the presented setup.

\subsubsection{TEM measurements}

Since the electron beam of the gun currently used in the ULEED setup samples a region of few tens of micrometers in diameter (Sec. 5.3), TEM as well as cryo-TEM measurements with few-nanometer sampling areas are performed to further investigate the nature of the superstructure (Fig. 6.8A). However, under irradiation of a regular TEM and even the low-dose approach of the cryo-TEM, a superstructure degradation within a few seconds is observed, making it challenging to perform more than a qualitative investigation on the system (see App. B.4).

Nevertheless, when sampling only few tens of nanometer large areas in a (cryo-) TEM in diffraction, three basic observations can be made:

1. Mainly two and sometimes four superstructure spots are visible out of the six in the images recorded with the ULEED setup (Fig. 6.8A).

2. Areas of single-domain superstructure abundance are about $10 \mathrm{~nm}$ in diameter.

3. The superstructure is only visible on about half of the investigated area. 

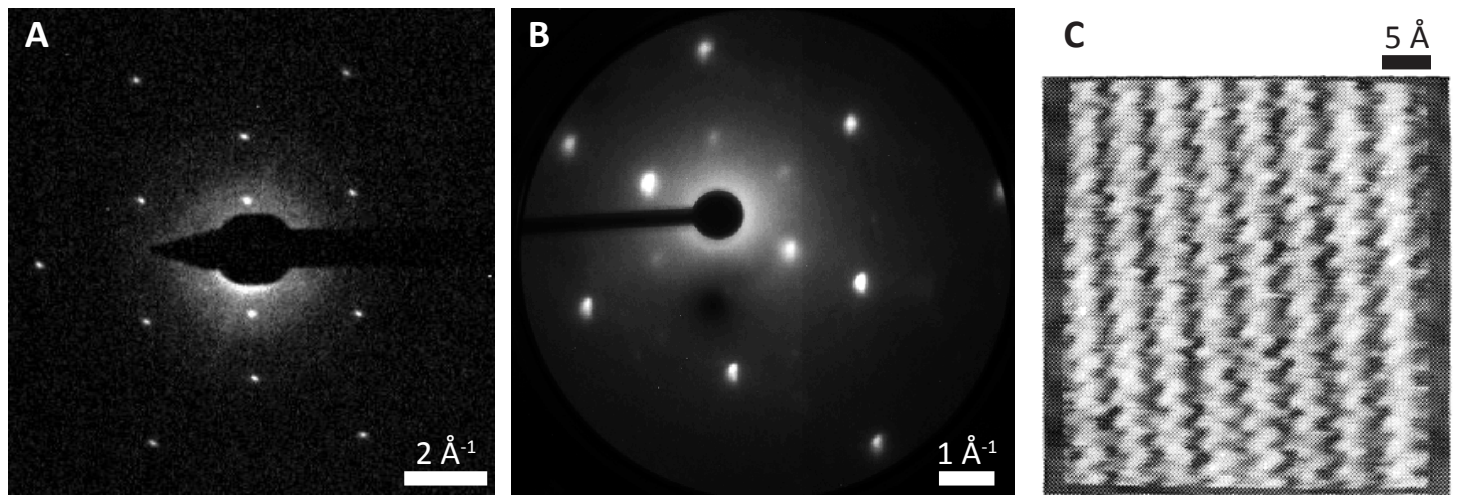

Figure 6.8: Characterization of the PMMA superstructure. A: Low-dose cryo-TEM image with few nanometer sampling area displays only two PMMA diffraction spots, indicating a stripe-like order as depicted in C. Electron energy: $300 \mathrm{keV}$. B: ULEED diffraction pattern of an infrequently encountered sample area showing a preferred direction of orientation (two bright center spots) of the adsorbed PMMA superstructure. C: STM image of atactic PMMA on HOPG with chain-to-chain distance of 5.0(1.0) $\AA$, taken from Ref. [110]. [229]

The first observation can be explained by the abundance of three superstructure domains with a periodicity in only one direction, instead of a hexagonally symmetric $2 \times 2$ superstructure (Sec. 2.2.2). As far as this observation goes, the domains differ only by being rotated by $60^{\circ}$ with respect to each other, while all of them are orientationally linked to the substrate.

The formation of stripe-like crystalline domains of polymers on various substrates has been previously observed. Specifically, AFM and STM measurements have been performed for various PMMA types on mica and highly ordered pyrolytic graphite (HOPG) [110, 111, 230, 231], finding tacticity-dependent configurations of so-called folded-chain crystals. Remarkably, this is also the case for atactic PMMA (Fig. 6.8C). Specifically, the charge density distribution recorded by the STM shows a strong periodicity along one direction, whereas the other direction displays a significantly weaker periodicity at a higher spatial frequency. However, this order along the backbone of the polymer is not visible in the ULEED diffraction images.

The second observation already gives a rough idea of the approximate domain dimension. Specifically, the domain size is of the same order as the transverse coherence length of the current ULEED setup. Taking into account a substrate coverage of approximately $50 \%$, it is likely that interference of diffraction signals from different domains plays only a minor role. Instead, the pattern can be seen as a superposition of the individual domains. 
Additionally, it can be concluded from the homogeneous intensity of the six individual superstructure spots in the majority of ULEED diffraction images (see e.g. Fig. 6.5B and $\mathrm{C}$ ) that the three domain orientations are uniformly distributed. Fig. 6.8B shows a ULEED diffraction image taken of an only rarely encountered sample region also displaying a preferred orientation of the superstructure, indicated by the higher intensity of two out of the six inner diffraction spots.

The last observation, concerning the PMMA coverage, agrees well with TEM images taken from the samples (Fig. 6.10C) and will become important when analyzing the out-of-equilibrium dynamics of the superstructure, in particular the expansion of the superstructure lattice.

\subsubsection{STM measurements}

To corroborate the notion of a folded-chain superstructure, STM measurements on the samples at a temperature of $77 \mathrm{~K}$ are performed. Before interpreting the results, it has to be noted that it was not possible to repeat individual measurements at the same position. This might stem from mobile PMMA chain segments, which got shifted by the STM tip. However, several observations are supporting the interpretation of a PMMA foldedchain crystal superstructure: Firstly, the measurements could be reproduced at different sample positions. Secondly, the instable tunneling process during the measurements indicates the abundance of a material overlayer other than graphene. Furthermore, the orientation of the stripe-like features fits the orientation of graphene and shows double its periodicity. And lastly, the obtained periodicity for both graphene and PMMA agree well with values found in the literature.

Figure 6.9A displays two exemplary STM images of PMMA (top) and graphene (bottom). The averaged traces of these images (area of averaging indicated by the dashed rectangular boxes) are plotted in Fig. 6.9B. By Fourier transformation, the periodicity of graphene is calculated to be $2.50 \AA$ (literature value: $2.46 \AA$ ), while the PMMA chain-to-chain distance is found to be $5.13 \AA$ (literature value: 5.0(1.0) $\AA$ [110]).

From these measurements, the polymer superstructure is understood to have the following configuration: The PMMA forms strands on top of the graphene displaying double periodicity with respect to the substrate's unit cell, $|\mathbf{b}|=2\left|\mathbf{a}_{\mathbf{1}}\right|=4.92 \AA$. It orders 

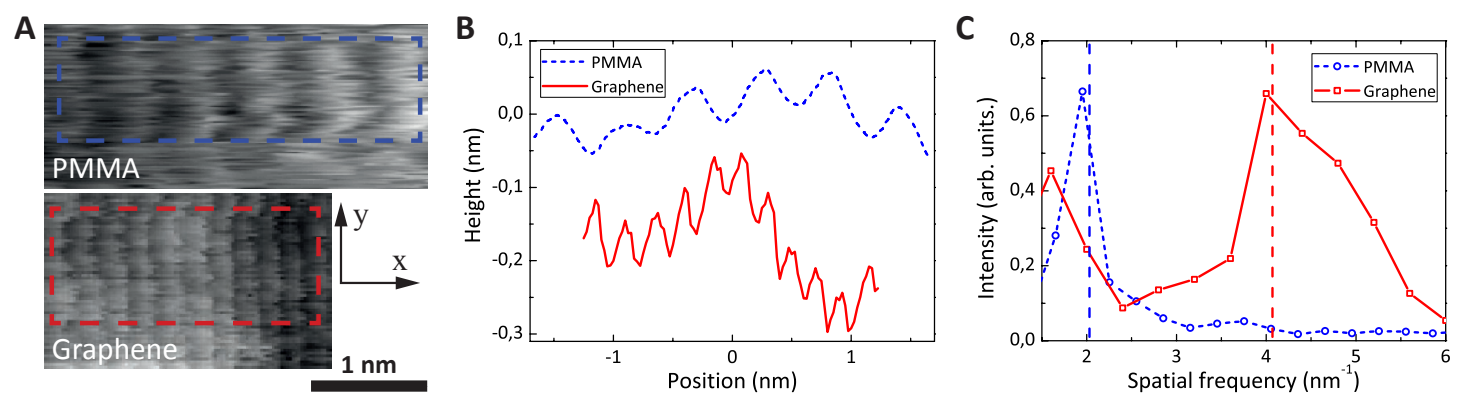

Figure 6.9: STM characterization of the PMMA/graphene bilayer. A: STM images of PMMA (top, voltage $-0.2 \mathrm{~V}$, constant current $0.2 \mathrm{nA}$ ) and empty graphene (bottom, voltage $0.25 \mathrm{~V}$, constant current $0.2 \mathrm{nA}$ ) displaying double periodicity of PMMA with respect to graphene in $x$ direction (black arrow). Dashed boxes indicate area of $y$-integration for B and C. Stripe-like appearance of graphene stems from preferred direction of scanning. Scale applies to both images. B: Integrated STM traces for both PMMA (dashed blue line) and graphene (solid red line). C: Fourier expansion of PMMA and graphene data in B, showing peaks at $1.95 \mathrm{~nm}^{-1}$ and $4.00 \mathrm{~nm}^{-1}$, respectively. Dashed lines indicate reciprocals of values given in the literature for the lattice spacing. [232]

itself in folded chains, which are following the directionality of the underlying graphene lattice, resulting in three different possible domain orientations rotated by $60^{\circ}$ with respect to each other. In particular, the adaptation to the substrate orientation follows from the periodically corrugated adsorption potential of graphene.

The size of these folded-chain polymer domains is estimated from TEM measurements (Fig. 6.8A) to be of the order of $10 \mathrm{~nm}$ in diameter, corresponding to about 20 chain spacings. This feature is in agreement with the typical correlation length, which is found for most of the samples probed to be of the order of 5 to 10 chain spacings. With the high molecular weight of the used PMMA (approx. $10^{4}$ monomers per chain), and assuming enough time for the molecule to rearrange on the graphene substrate, this results in a single PMMA chain building up to 50 connected crystal superstructure domains.

Since the samples are stored at temperatures below the glass transition temperature of the atactic PMMA $\left(T_{G} \approx 105{ }^{\circ} \mathrm{C}\right)$, its chain mobility is strongly decreased. Hence, we assume that despite the long drying time, at least a small part of the PMMA is still in amorphous form, visible as background signal in the diffraction pattern. In agreement with this assumption, the superstructure signal quality increased for samples, which have been subjected to longer drying times (see App. B.2).

Nevertheless, as the polymer superstructure diffraction peak intensity has been found to be comparable with the one of graphene and does not change significantly when scanning 
the sample in ULEED, considerable overall crystallinity and uniform substrate coverage on the micrometer scale can be inferred.

An exemplary PMMA superstructure on the graphene substrate is sketched in Fig. 6.10A and B. The red and orange tubes are showing two different folded-chain domains, both aligned to the graphene substrate and rotated by $60^{\circ}$ with respect to each other (Fig. 6.10A). For a more detailed picture, a magnified PMMA folding is displayed in comparison to the graphene unit cell in Fig. 6.10B.
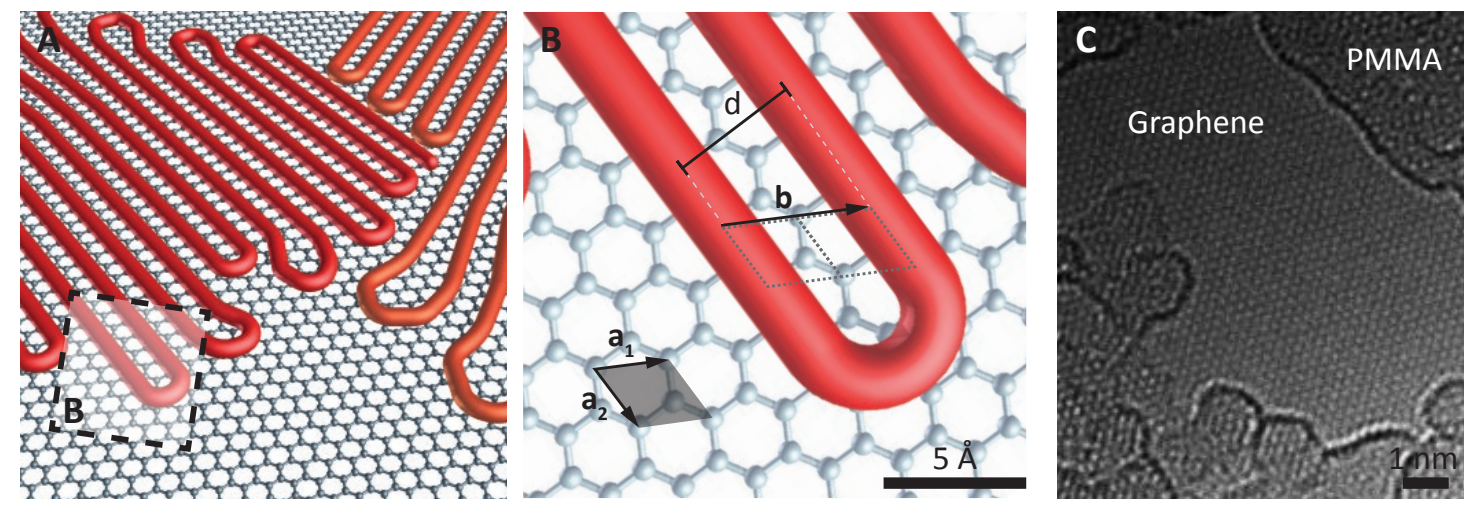

Figure 6.10: PMMA superstructure sketches and TEM image. A: Schematic sketch of the ordered PMMA chains (red and orange) on the graphene substrate. Dashed rectangle indicates area magnified in B. B: Graphene unit cell (gray area) with lattice vectors $\mathbf{a}_{i}$. Double periodicity $|\mathbf{b}|=4.92 \AA$ of superstructure with respect to (dashed) graphene unit cell and chain-to-chain distance $d=4.26 \AA$. C: TEM image of typical sample at $80 \mathrm{keV}$, displaying graphene and unordered PMMA. [233]

For comparison, a TEM image also displaying both graphene and PMMA, is depicted in Fig. 6.10C. In this case, however, any existing PMMA superstructure has been already destroyed by intense electron beam irradiation (see App. B.4). As mentioned above, the total PMMA coverage of graphene is of the order of $50 \%$ (more TEM images found in Ref. [103, 105, 223]).

As can be seen in the diffraction images (e.g. Fig. 6.5C) as well as in Fig. 6.10B, the inter-chain spacing of PMMA is determined by Eq. 2.3:

$$
d=\frac{2 \pi}{a_{p}^{*}}=4.26 \AA .
$$

With graphene having a hexagonal lattice with lattice vectors of length $\left|\mathbf{a}_{i}\right|=2.46 \AA$, a double periodicity results in a value of $|\mathbf{b}|=2\left|\mathbf{a}_{\mathbf{1}}\right|=4.92 \AA$, because the PMMA chain-to-chain vector is not parallel to the unit cell vector of graphene. However, when discussing the superstructure lattice parameter, the value of $d=4.26 \AA$ is referred to. 


\subsubsection{Higher diffraction orders}

Upon increase of the electron energy, higher diffraction orders of graphene and the superstructure become visible (Fig. 6.11A and B). In case of the latter, however, the diffraction spot intensity decreases rapidly with increasing order, which has been previously reported for similar systems, for example in electron diffraction experiments of polyethylene folded-chain crystallites [234].
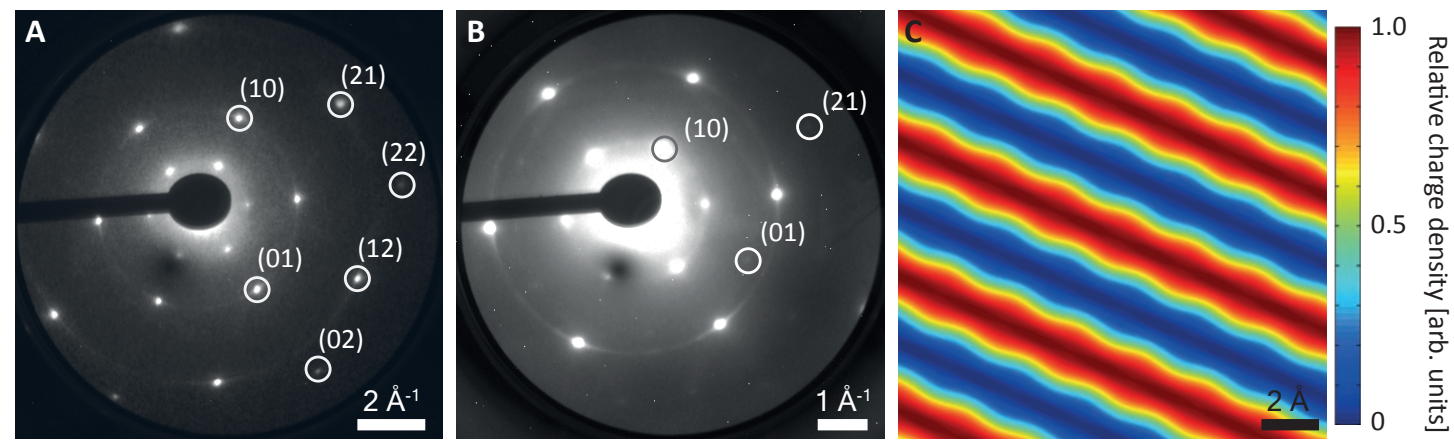

FiguRE 6.11: Higher diffraction orders of graphene and superstructure, simulated superstructure charge density modulation. Numbers denote Miller indices in graphene (A) and superstructure (B) lattice units. Electron energy: $1000 \mathrm{eV}(\mathrm{A}), 500 \mathrm{eV}$ (B). Integration time: $1.5 \mathrm{~s}(\mathrm{~A}), 45 \mathrm{~s}(\mathrm{~B})$. A: Diffraction image with graphene and PMMA peak labels. B: Diffraction peaks correspond to the same superstructure domain orientation. C: Idealized charge density modulation simulated for spot intensities and positions as found in B.

For illustration purposes, an idealized charge density distribution is simulated in the following, not taking into account static or dynamic disorder. While the position and shape of the diffraction spots is given by the relative arrangement of the unit cells and hence the structure factor $|S|^{2}$, their intensity $I$ is ultimately determined by the atomic form factor $|F|^{2}[69]$. Specifically, the atomic form amplitude is the Fourier transform of the charge density distribution $\rho$ within the scattering unit cell,

$$
I \propto\left|F_{h k}\right|^{2}=\rho^{2}
$$

Using Eq. 6.2, the relative atomic form factor amplitudes $F_{h k}$ can be evaluated from the measured spot intensities $I_{h k}$ in Fig. 6.11B. For a computation of the absolute value, however, the intensity of the zeroth order (transmitted) beam would have to be known. Specifically, the spot intensity is determined by subtracting a linear, radius-dependent background and a subsequent fit with a 2D Gaussian. 
The charge density distribution $\rho(x, y)$ is now obtained via the 2D Fourier series [235]

$$
\begin{aligned}
\rho(x, y) & \propto \sum_{h} \sum_{k} \varphi_{h k} F_{h k} \exp \left(i\left(k_{h} x+k_{k} y\right)\right) \\
& \propto \varphi_{10} F_{10} \sin \left(k_{h} x\right)+\varphi_{01} F_{01} \sin \left(k_{k} y\right)+\varphi_{21} F_{21} \sin \left(2 k_{h} x\right) \sin \left(k_{k} y\right),
\end{aligned}
$$

where the $\varphi_{h k}= \pm 1$ denote the phase coefficients and, without loss of generality, $\rho(x, y)$ was assumed to be an odd function. The magnitudes of the wave vectors $k_{h}$ and $k_{k}$ in the [10] and [01] directions, respectively, are obtained from Fig. 6.11B.

The result for $\rho(x, y)$ is depicted in Fig. 6.11C for $\varphi_{h k}=1$. Due to the symmetry of Eq. 6.4, there are only two distinguishable, yet still similar modulations for the overall eight combinations of the $\varphi_{h k}$ [191]. Specifically, Fig. 6.11C shows a strong modulation of the charge density along the [10] direction and a weaker modulation perpendicular to it. When interpreting this result, one has to keep in mind that Eq. 6.4 is based upon a strongly simplified picture of the physical system, e.g., using the Born approximation, a kinematic treatment (see also Sec. 6.2.6), and, more importantly, neglecting the influence of disorder. Nevertheless, one can conclude that this rough analysis is in support of previous results in terms of the polymer conformation on the graphene (compare Fig. 6.8C).

\subsubsection{Bilayer thickness}

Qualitatively, it can be reasoned that the strong template properties of the graphene only result in superstructure formation on the PMMA layer in direct contact with it [221]. Hence, possible subsequent PMMA layers may likely be non-crystalline and therefore only contribute to the scattering intensity as an amorphous background.

However, due to the small penetration depth of low-energy electrons, a rough estimate of the average bilayer thickness can be performed by means of the IMFP at those energies, when the signal drastically decreases (compare Sec. 6.2.1). This decrease is found at about $240 \mathrm{eV}$, corresponding to an IMFP of around $6 \AA$, which is in good agreement with the assumed monolayer coverage of PMMA. When approximating the PMMA strains as tubes with constant diameter, then their diameter cannot be larger than the chain-tochain distance, which is about $4.3 \AA$. Additionally, the graphene thickness is estimated 
by the inter-plane distance of successive graphene planes, which was determined to be about $4 \AA[72]$, resulting in a upper limit for the total bilayer thickness of about $8 \AA$.

In surface diffraction, multiple scattering from successive crystal planes has to be considered, which increases the level of complexity to quantitatively model the surface structure [6]. However, this effect is usually negligible in transmission studies of the ultrathin bilayers as in the present case.

\subsection{Ultrafast relaxation dynamics of the PMMA super- structure}

In the following, a quantitative analysis of the reversible relaxation of the presented bilayer system is given with focus on the superstructure dynamics, when excited farout-of equilibrium. Specifically, the current ULEED setup is employed to resolve the ultrafast temporal evolution of the different processes taking place after intense laser irradiation.

First, the general observation of an intensity loss of the diffraction spots is discussed (Sec. 6.3.1). In the next step, differently behaving components of the superstructure are identified (Sec. 6.3.2). Then, the temporal evolution of processes connected to the superstructure relaxation are mapped and their characteristic time constants determined (Sec. 6.3.3). Lastly, the graphene spot evolution is investigated (Sec. 6.3.6) and a physical picture is derived and discussed (Sec. 6.4), followed by the conclusions (Sec. 6.5).

\subsubsection{Diffraction intensity reduction}

Figure 6.12 displays the change in diffraction intensity of graphene and the PMMA superstructure, when illuminated by an intense laser pulse (pump pulse, duration: 3 ps, fluence: $6 \mathrm{~mJ} / \mathrm{cm}^{2}$ ) before (B) and after (C) arrival of the pump pulse as well as without excitation (A).

The diffraction image of the unpumped sample (Fig. 6.13A) shows the hexagonal symmetric peaks of the graphene as well as three pairs of peaks of the adsorbed PMMA superstructure with comparable intensity (Sec. 6.2). At negative delay times $(\Delta t<0 \mathrm{ps,}$ 

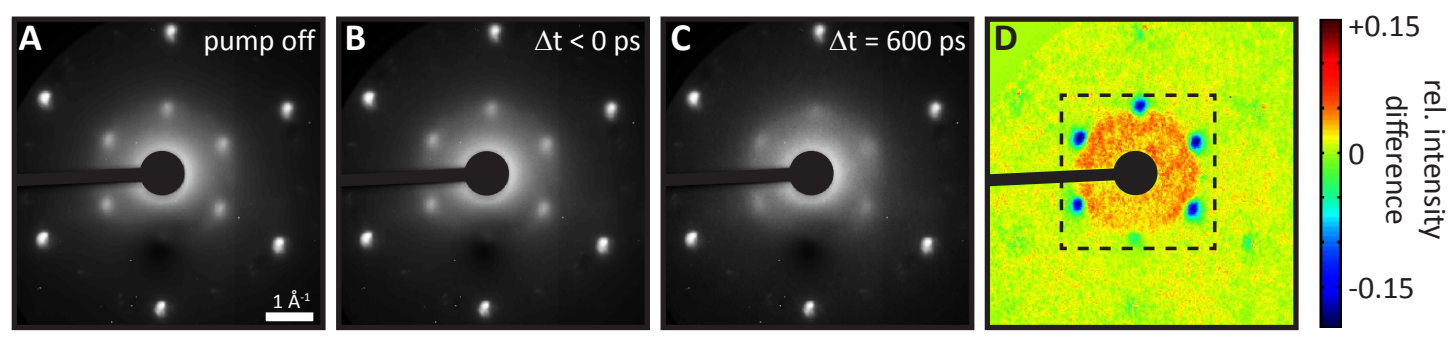

FiguRE 6.12: Change in the diffraction images upon laser irradiation. Electron energy: $450 \mathrm{eV}$. Pump fluence: $6 \mathrm{~mJ} / \mathrm{cm}^{2}$. A-C: averaged diffraction images for unpumped sample (A), pumped sample at negative (B) and positive (C) delay times. D: Difference map of images B and C. Dashed rectangle denotes image area shown in Fig. 6.13A.

electron probe pulse arrives before laser pump pulse), the intensity of the superstructure spots is slightly decreasing by about 10-15\% with respect to the unpumped sample (cf. Fig. 6.13A). This can be attributed to a non-complete recrystallization of the superstructure between two pump pulses. With the repetition rate of $10 \mathrm{kHz}$ used in the experiment, the recrystallization time is therefore of the order of below $100 \mu \mathrm{s}$.

The intensity decrease is significantly amplified at long positive delay times (Fig. 6.12C, $\Delta t=600 \mathrm{ps}$, electron probe pulse arrives after laser pump pulse), whereas the graphene spot intensity stays seemingly constant. The intensity development can be best analyzed by computing the difference between images taken at positive delays with respect to images taken at negative delays (Fig. 6.12D).

In these difference maps, a further observation can be made: Next to the loss of intensity for the superstructure peaks (blue), a slightly higher intensity at smaller scattering angles is observed (red disc). Notably, this increase does not show a preferred direction of orientation.

\subsubsection{Amorphous and crystalline components}

For a further examination of the temporal evolution, the difference maps for three delay times are analyzed with respect to their changes in diffraction intensity.

Figure 6.13A displays the small angle scattering region (as denoted by the black dashed rectangle in Fig. 6.12D) for three positive pump-probe delays. Mainly two features are observed, namely the already mentioned reduction of the superstructure diffraction peak intensity and an intensity increase at small scattering angles, corresponding to an inplane momentum transfer of $k_{\|}<1.25 \AA^{-1}$. As can be seen in $(i)$ and $(i i)$, the spot 


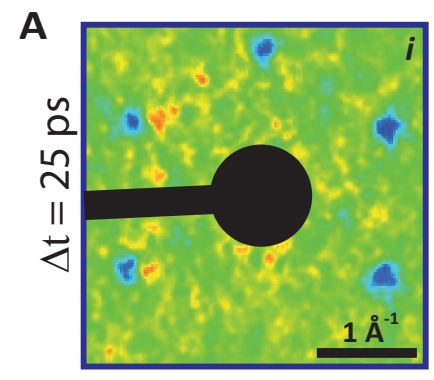

B

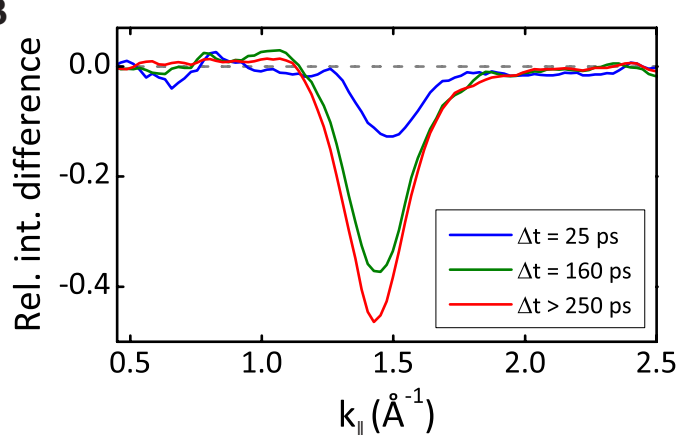

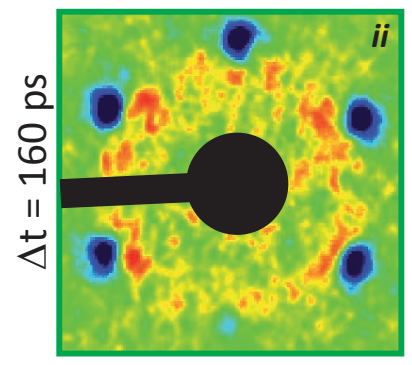

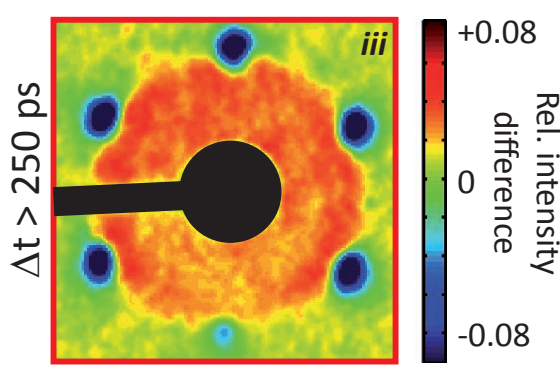

C

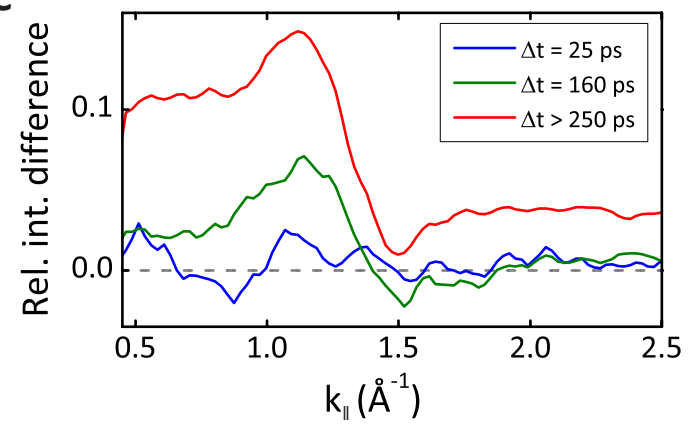

Figure 6.13: Difference maps of superstructure diffraction images. Parameters as in Fig. 6.12. A: Superstructure diffraction images for three different points in time, image area denoted by dashed rectangle in Fig. 6.12D. Blue (red) color indicates intensity decrease (increase). B: Momentum-dependent peak intensity evolution without isotropic contribution for delay times denoted in A. C: As in B, but only intensity of small-angle scattering without peak contribution.

intensity decrease takes place on a different time scale with respect to the formation of the inner disc $(i i i)$.

For a separate analysis of the individual features, the angularly averaged spot intensity without the isotropic contribution from the disc is displayed in Fig. 6.13B. Likewise, the latter is analyzed in Fig. 6.13C without the spot contribution. A detailed account on how these components are separated is given in App. A.5.

Interestingly, the isotropic feature displays a peak in addition to the plateau region visible in the difference maps (Fig. 6.13B A (iii)). The intensity change is maximal at $1.12 \AA^{-1}$, indicating a preferred spatial correlation length of about $5.6 \AA$, which is approximately $25 \%$ larger than the lattice parameter of the crystalline state.

Additionally, the notion of different time scales can be consolidated upon comparison of the intensity changes at the same point in time relative to the respective feature's peak value at large delays: Whereas the intensity loss of the spots (Fig. 6.13B, $k \approx$ $1.5 \AA^{-1}$ ) is nearly complete after about $160 \mathrm{ps}$, only about half of the maximal intensity increase has been achieved in case of the extended amorphous structure (Fig. 6.13C, 
$k \approx 1.2 \AA^{-1}$ ). Additionally, the time scale for the latter is apparently strongly dependent on the scattering angle. This feature can be confirmed by relating the displayed intensity change to the scattering momentum. Explicitly, the characteristic time scale is increasing with decreasing $k_{\|}$.

\subsubsection{Superstructure relaxation time scales}

For a quantitative determination of the underlying characteristic time scales, a detailed delay-dependent measurement with a temporal step-width between 10 ps (around timezero) and $60 \mathrm{ps}$ (for long delay times) is performed. In Fig. 6.14A, the evolution of the superstructure diffraction peak (blue triangles) and disc (orange circles) intensity is displayed and fitted (solid lines with respective colors, fitting method detailed in App. A.4). In case of the peak intensity evolution, a characteristic time scale of 105(8) ps is found, whereas the isotropic intensity changes on the order of 228(61) ps. The relative changes in amplitudes are $-40 \%$ and $+12 \%$, respectively.
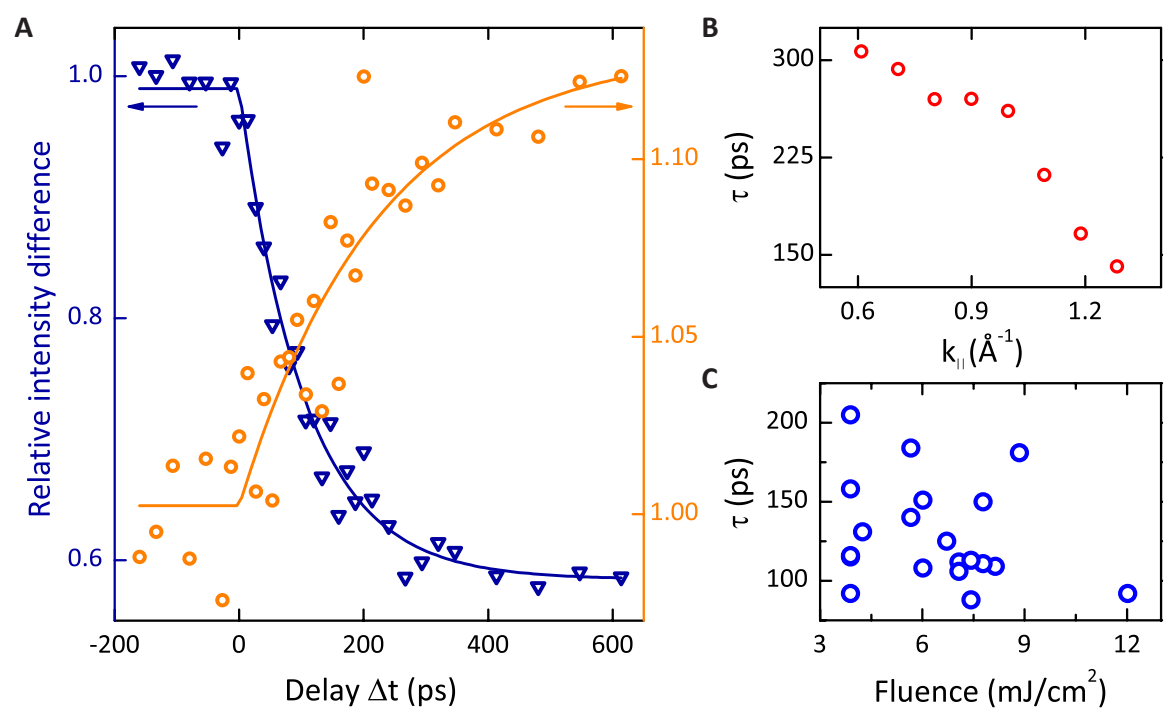

Figure 6.14: Structural evolution of the superstructure relaxation. A: PMMA diffraction peak (blue triangles) and small angle scattering (orange circles, integrated over all scattering angles) intensity development. Fluence: $6 \mathrm{~mJ} / \mathrm{cm}^{2}$. B: Scattering momentum dependent time constants for small angle scattering. C: Time constants as a function of laser pump fluence.

Notably, the value of approximately $230 \mathrm{ps}$ is obtained, when integrating the difference maps over a large range of scattering momenta between $0.6 \AA^{-1}$ and $1.3 \AA^{-1}$. Yet, as observed in Fig. $6.13 \mathrm{~B}$ and $\mathrm{C}$, the determined duration is a strong function of the 
scattering angle. To quantify this observation, the obtained time constants are displayed as a function of scattering momentum, using a integration width of $0.1 \AA^{-1}$, in Fig. 6.14B. Specifically, the computed characteristic time constants range from 150 ps $\left(k_{\|}=1.3 \AA^{-1}\right)$ to values beyond $300 \mathrm{ps}\left(k_{\|}<0.6 \AA^{-1}\right)$.

The functional dependence can be used to define a characteristic velocity $v$ of the process related to the small angle scattering increase:

$$
v=\frac{\Delta \lambda}{\Delta \tau}=\frac{2 \pi}{\Delta k \Delta \tau} \approx 6 \frac{m}{s}
$$

Notably, this velocity is significantly smaller than the speed of sound in PMMA $\left(v_{s}=\right.$ $2780 \mathrm{~m} / \mathrm{s},[236]$ ). We will return to this observation at the end of this chapter (Sec. 6.4).

Furthermore, a possible correlation between the observed time constant and the employed fluence level is investigated. To this end, the superstructure dynamics are recorded for numerous samples prepared under the same conditions, yielding a mean characteristic time scale for the peak intensity loss of 128(32) ps. The individual time constants are displayed in Fig. 6.14C as a function of employed laser pump fluence. So far, within the sample-to-sample variability, we found no fluence-dependency of the observed time constants for fluences between $3 \mathrm{~mJ} / \mathrm{cm}^{2}$ and $12 \mathrm{~mJ} / \mathrm{cm}^{2}$.

\subsubsection{Fluence threshold}

Interestingly, for fluence levels below $3 \mathrm{~mJ} / \mathrm{cm}^{2}$, the diffraction intensity loss of the peaks is found to be strongly suppressed. On these grounds, fluence-dependent measurements of the superstructure diffraction peak intensity and position are conducted (Fig. 6.15A). Up to a threshold fluence of about $3 \mathrm{~mJ} / \mathrm{cm}^{2}$, the diffraction peak intensity is slightly decreasing with increasing pump fluence, e.g., due to heating of the sample (blue squares, see Sec. 6.3.6). Above the threshold fluence, the intensity loss is strongly enhanced, displayed by a kink in the otherwise linear behavior. This finding is paralleled by an expansion of the superstructure lattice, which is also significantly steepened above the same critical fluence. Explicitly, the superstructure lattice expansion amounts to about $1 \%(0.05 \AA)$ at the threshold, while a doubling of the pump fluence further increases the lattice parameter by an additional $5 \%(0.2 \AA)$. 

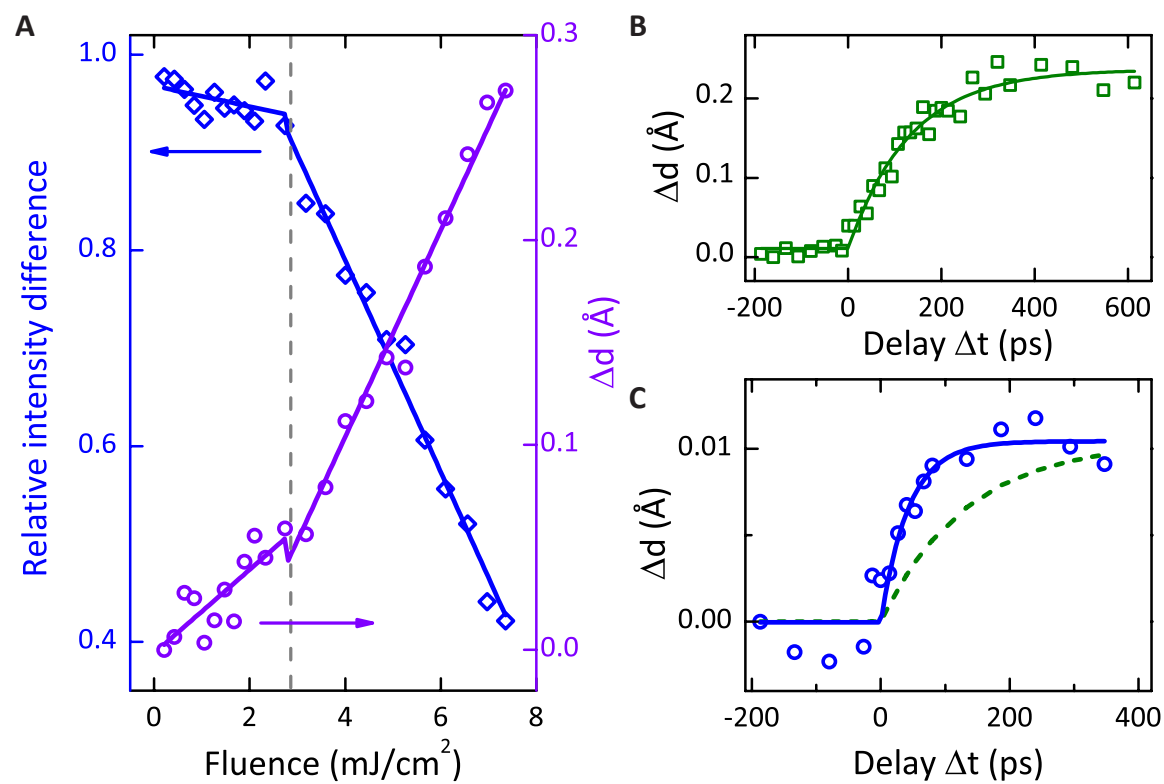

Figure 6.15: Fluence dependent intensity and spot position measurements. Electron energy: $450 \mathrm{eV}$. A: Superstructure peak (turquoise diamonds) and lattice parameter (purple circles) development as a function of pump fluence at large delay times $(\Delta t=$ $600 \mathrm{ps}$ ). Turquoise and purple lines are linear fits to the experimental data and should serve as a guide to the eye. B, C: Delay-dependent superstructure lattice parameter expansion above $(\mathrm{B})$ and below $(\mathrm{C})$ the fluence threshold with respect to the unpumped value $(d=4.26 \AA)$. Pump fluences: $6.0 \mathrm{~mJ} / \mathrm{cm}^{2}$ and $1.9 \mathrm{~mJ} / \mathrm{cm}^{2}$, respectively. Green dashed line in $(\mathrm{C})$ indicates time constant found in $\mathrm{B}$, scaled to the amplitude observed in $\mathrm{C}$.

The relatively abrupt change of the superstructure in crystallinity and lattice parameter upon passing the threshold fluence are indicating the abundance of two qualitatively different states of the polymer superstructure. The unexcited state comprises a foldedchain conformation with a well-defined lattice parameter, a high degree of order and an orientationally linkage to the substrate. After laser excitation, the order is strongly reduced and the formation of components with an expanded lattice parameter and no further registration to the graphene is observed.

The initial temperature increase of the graphene lattice up to the fluence threshold is estimated by assuming that the specific heat capacity $c_{G}$ of graphene equals that of graphite for temperature $T>100 \mathrm{~K}$ [237]. Specifically, it can be approximated by the following polynomial for $200 \mathrm{~K}<T<1200 \mathrm{~K}$ [238]:

$$
\begin{aligned}
c_{G} & =9 \cdot 10^{-16} T^{6}-6 \cdot 10^{-12} T^{5}+2 \cdot 10^{-8} T^{4} \\
& -2 \cdot 10^{-5} T^{3}+0.0092 T^{2}+0.9463 T-10.481\left[\frac{\mathrm{J}}{\mathrm{kg} \mathrm{K}}\right] .
\end{aligned}
$$


With the PMMA being quasi-transparent at the pump wavelength of $800 \mathrm{~nm}$, the energy of the pump pulse is mainly deposited into the graphene (2.3\% absorption, see [239]). At a fluence level of $3 \mathrm{~mJ} / \mathrm{cm}^{2}$, we obtain an initial graphene lattice temperature increase of 536 K. Subsequently, the thermal energy is transferred to the PMMA though the bilayer interface, leading to the observed expansion of the lattice parameter. The characteristic time for this process is discussed in the next section.

The temperature of the superstructure at the fluence threshold can be computed by means of the thermal expansion coefficient. Specifically, for a thermal expansion coefficient of $\alpha=7 \cdot 10^{-5} \mathrm{~K}^{-1}$ of bulk PMMA [240], the temperature at the fluence threshold is calculated to be about $165{ }^{\circ} \mathrm{C}$. This is close to the reported melting temperature range of values between $130{ }^{\circ} \mathrm{C}$ and $160{ }^{\circ} \mathrm{C}$ (Sec. 6.1.1).

\subsubsection{Energy transfer time}

The temporal evolution of the superstructure lattice expansion is displayed in Fig. 6.15B, exhibiting a characteristic time constant of about 133(13) ps. Remarkably, the time scale of the expansion is drastically reduced below the threshold fluence found in Fig. 6.15A $(\tau=43(10) \mathrm{ps})$. For a better comparison, Fig. 6.15C displays the delay-dependent superstructure peak position (blue circles and line) as well as the characteristic time constant found for the lattice expansion above the fluence threshold (green dashed line), normalized to the same amplitude.

In order to determine, if the time constant of the observed lattice expansion is limited by the mechanical properties of PMMA, the time of a sound wave traveling the length of a single superstructure domain is computed. Assuming that the velocity of sound of crystalline PMMA is of the same order as in bulk $\left(v_{s}=2780 \mathrm{~m} / \mathrm{s}\right)$ and taking into account the approximate domain size of $10 \mathrm{~nm}$ of the superstructure (Sec. 6.2), a time duration of less than 4 ps is obtained. Since also the temporal resolution of the ULEED setup is about one order of magnitude higher than the measured value of $\tau=43 \mathrm{ps}$, the latter is therefore attributed to the characteristic time scale for the energy transfer across the bilayer interface from the graphene to the PMMA.

Alternatively, the energy transfer time across the bilayer interface can also be calculated using the thermal boundary conductance of at an carbon / polymer interface [241]. 
It has to be considered, however, that the heat flow across an interface is drastically reduced compared to bulk diffusion [242]. Specifically, the temperature decay in a thin film on a substrate is given by [134]

$$
c_{p} \rho_{p} d_{p} \frac{\partial T_{p}(t)}{\partial t}=-\frac{T_{p}(t)-T_{G}(t)}{R_{k}}
$$

with $c_{p}$ the specific heat capacity of the film, $d_{p}$ its thickness, $R_{k}$ the thermal boundary (Kapitza) resistance of the substrate-film system, $\rho_{p}$ the film mass density and $T_{p}$ and $T_{G}$ the film and substrate temperatures, respectively. When assuming temperatureindependent material constants, the energy transfer time across the interface is then given by

$$
\tau=R_{k} c_{p} d_{p} \rho_{p}
$$

In the present case, the following values were used: $c_{p, \text { PMMA }}=1466 \mathrm{~J} / \mathrm{kgK}$ and $\rho_{p, \text { PMMA }}=$ $1.18 \mathrm{~g} / \mathrm{cm}^{3}$ (Sec. 6.1.1) as well as $R_{k}=8 \cdot 10^{-8} \mathrm{~m}^{2} \mathrm{~K} / \mathrm{W}$ for a polymer / carbon nanotube (CNT) interface [243, 244]. Together with the PMMA film thickness previously estimated to be $d_{p, \text { PMMA }}=4.3 \AA$ (Sec. 6.2.6), we obtain a transfer time of $\tau \approx 60$ ps, close to the experimentally determined value. However, since no experimental data on the thermal boundary resistance at a graphene / polymer interface could be found in the literature, the respective value in case of a polymer / CNT interface was used. However, theoretically obtained values for $R_{k}$ from molecular dynamics simulations of graphene / polymer systems are available, resulting in a somewhat smaller characteristic time constant of $\tau \approx 30 \mathrm{ps}$ instead for $R_{k}=4.0(1.0) \cdot 10^{-8} \mathrm{~m}^{2} \mathrm{~K} / \mathrm{W}$ [245]. For comparison, the Kapitza resistance of the PMMA / graphene interface computed from the experimentally observed time constant in the present measurement is $R_{k}=5.8 \cdot 10^{-8} \mathrm{~m}^{2} \mathrm{~K} / \mathrm{W}$.

\subsubsection{Graphene spot evolution}

Upon careful investigation of the difference maps, a slight reduction of the graphene spot intensity by $1-2 \%$ is visible (Fig. 6.16A). In the following, this observation is investigated in view of three potential causes, namely a decreased scattering efficiency by lattice warming (Debye-Waller effect), electron deflection by a laser pump pulsegenerated TEF effect, and superimposed higher order superstructure diffraction spots. 

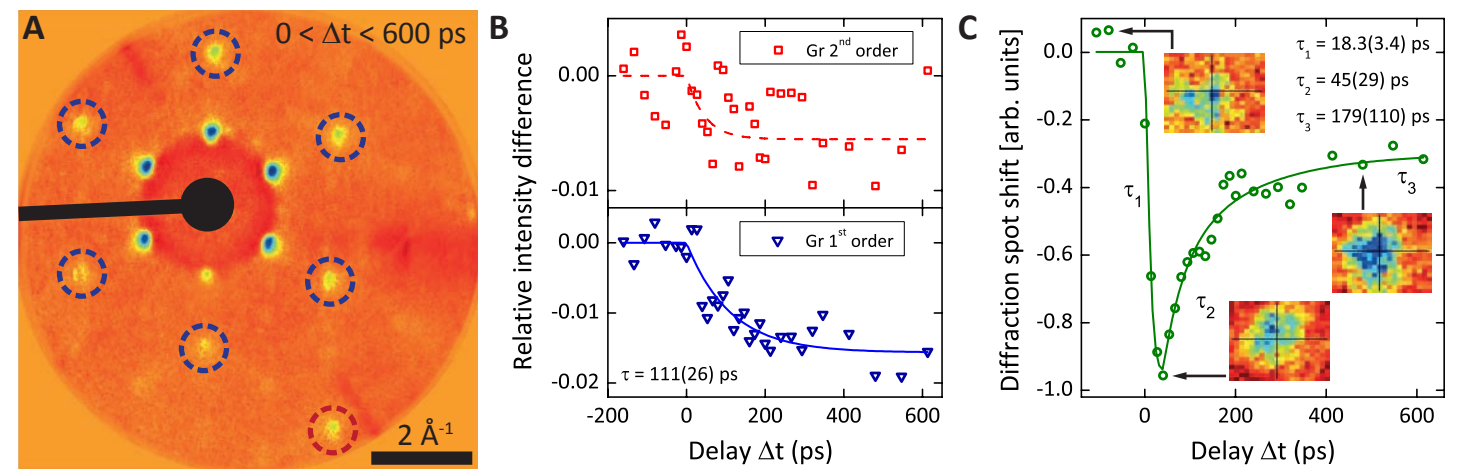

Figure 6.16: Graphene spot dynamics. A: Difference image between pumped and unpumped sample shows the intensity change of first (blue circles) and second (red circle) order graphene spots when integrated over all positive delay times. B: Delaydependent intensity change of graphene spots for first (lower panel) and second (upper panel) order. C: Delay-dependent graphene spot shift. Insets: Integrated diffraction spot images at marked delays. Fluence: $6 \mathrm{~mJ} / \mathrm{cm}^{2}$, electron energy: $450 \mathrm{eV}$.

In case of graphene, the derivation of the Debye-Waller factor $\exp ^{-2 W}$ is somewhat challenging, since it diverges for an infinite, two-dimensional crystal at temperatures above zero [246-249]. For this reason, two-dimensional materials were believed to be inherently thermally unstable before their experimental realization by A. Geim and K. Novoselov in 2004 [71]. However, it is possible to extract an approximate solution for temperatures above absolute zero, when the finite size of the crystal is taken into account $[250,251]$ :

$$
2 W \approx G^{2}\left[\frac{\hbar}{k_{D} M v_{s}}+\frac{2 k_{B} T}{k_{D}^{2} M v_{s}^{2}} \log \left(\frac{k_{B} T}{\hbar v_{s} k_{s}}\right)\right] .
$$

In Eq. $6.9, G$ is a discrete reciprocal lattice vector (taken as $2 \pi / a$ with $a=2.46 \AA$ the graphene lattice constant), $\hbar$ is Planck's constant, $M$ the carbon atom mass, $v_{s}$ the sound velocity in graphene $\left(2.4 \cdot 10^{4} \mathrm{~m} / \mathrm{s}[250]\right), k_{D}$ the Debye wave vector, $k_{B} T$ the thermal energy and $k_{s}$ smallest possible wave vector supported by the finite lattice ${ }^{10}$. For an estimate of the latter, $k_{s}=2 \pi / l_{c}$ with $l_{c}=5 \mathrm{~nm}$ the transverse coherence length of the experimental setup, as previously estimated in section (Sec. 6.2).

A fluence level of $6 \mathrm{~mJ} / \mathrm{cm}^{2}$ results in an initial graphene temperature increase of $746 \mathrm{~K}$ (Sec. 6.3.4), leading to an intensity reduction of $1.15 \%$ for the first order and $0.62 \%$ for the second order graphene diffraction peaks, which is well in the range of observed values. However, this initial lattice heating would happen on a very fast time scale of

\footnotetext{
${ }^{10}$ In the limit of an infinite lattice, $k_{s} \rightarrow 0$, and the diverging behavior of the Debye-Waller factor is recovered.
} 
the order of the pump pulse duration and should leave a visible trace in a time-resolved intensity analysis.

Figure 6.16B depicts the recorded relative intensity difference as a function of delay for the first order (lower panel, blue circles in Fig. 6.16A) and second order (upper panel, red circle in Fig. 6.16A) graphene spots. Only a single second order spot could be analyzed, since the detector distance and electron energy is optimized for the observation of the PMMA peaks in this measurement. In case of the first order graphene spots, a reduction of the intensity by about $1.5 \%$ (about $0.5-1.0 \%$ for second order) is visible at long delay times with a decay time of around 110 ps. A feature with a time constant in the range between the pump pulse duration ( $3 \mathrm{ps}$, heating of the graphene) and the energy transfer time (43 ps, thermal equilibration with the PMMA, hence graphene cooling) is not observed. Yet, with a temporal step width of about 10 ps around time-zero and a small overall signal intensity, it is possible that the fast Debye-Waller intensity transient is only partially recorded or hidden within the recorded transient. Lastly, at longer delay times, when the graphene and PMMA temperatures have equilibrated, the temperature of the combined bilayer is probably significantly lower, hence the reduction of scattering intensity less pronounced $\left(<0.2 \%\right.$ for first order and $T=T_{m \text {,PMMA }} \approx 160{ }^{\circ} \mathrm{C}$ ).

A missing fast intensity signal at small delay times also excludes a strong contribution to the observed spot intensity change by a TEF effect after optical pumping of the sample (Sec. 5.3.2). Even though an intensity transient is not detected, a small spatial shift of the graphene spots in one direction is observed, amounting to only about one pixel on the CCD of the camera (insets of Fig. 6.16C). For comparison, the measured magnitude of the superstructure spot shift as depicted in Fig. 6.15B is about three times larger. Figure 6.16C shows the averaged position of all six first order graphene diffraction spots as a function of delay time. Specifically, after a strong initial shift $\left(\tau_{1}\right)$, the graphene spots relax back towards their original position more slowly $\left(\tau_{2}\right)$. However, a full relaxation does not take place on the observed time scale. Instead, after reaching about $40 \%$ of the peak signal intensity, the spots shift evolution towards the initial state is strongly slowed $\left(\tau_{3}\right)$.

The observed fast transient can be attributed to a deflection of the electron pulse by a plasma cloud generated during sample excitation, which leads to a unidirectional shift of the diffraction pattern. It has to be noted that while this effect is well visible in case 
of the graphene spots, it does not influence the PMMA superstructure spot analysis due to the aforementioned unidirectionality. In this picture, the slower transient would be caused by a dissipation of the plasma cloud with relatively low-energy electrons. Similar behavior, i.e. 100-ps scale relaxations transients, are sometimes observed during pulse duration measurements [202]. Considering that PMMA is an insulator, the plateaus regions could be an indicator for remaining charge, which is only slowly dissipating over the bilayer interface into the sample holder.

For an estimate of the governing time scales, the signal has been fitted (solid green line) with a Gaussian error function with decay constant $\tau_{1}$, as well as with two single exponential decays with time constants $\tau_{2}$ and $\tau_{3}$. The resulting time scales are $\tau_{1}=18 \mathrm{ps}$, $\tau_{2}=45 \mathrm{ps}$, and $\tau_{3}=180 \mathrm{ps}$. Notably, the observed decay time $\tau_{1}$ is about one order of magnitude larger compared to those of TEF transients recorded during pulse duration measurements under similar conditions. This can be understood as the interplay of various effects: A small contribution is likely caused by the longer pump pulses compared to those in the pulse duration measurements, namely 3 ps instead of 80 fs. More importantly, the time scale of plasma cloud dissipation is strongly fluence-dependent (Sec. 5.3.2), leading to significantly slower dynamics at low fluences as employed in the measurements presented in Fig. $6.16\left(6 \mathrm{~mJ} / \mathrm{cm}^{2}\right)$. Also, TEF measurements are usually performed on blank $\mathrm{Cu}$ grids to avoid possible charging of insulating materials, such as the employed polymer. Lastly, the pulse duration measurements have been performed for a partial beam to reduce the influence of the plasma cloud dynamics. An integration over the whole area resulted in a transient of about 6 ps compared to 2 ps for the partial beam at $450 \mathrm{eV}$ electron energy. In this view, the unidirectional shift of the spots as well as the recorded time constant seem consistent with a pump-induced TEF.

A final remark on the graphene spot shift in Fig. 6.16C: the apparent oscillation of the signal after about 100 ps likely originates from a technical problem of the recording software used at the time of the measurement. For a further investigation of a possible physical effect, experiments with focus on the graphene spot shift and longer integration times are going to be performed in the near future.

An alternative explanation for the observed intensity reduction on a time scale of $110 \mathrm{ps}$ is based on higher order superstructure diffraction spots superimposed on the graphene spots. Specifically, the reduction in spot intensity of the superstructure occurs on a time 
scale of 108 ps (Sec. 6.3.3), which agrees well with the observed transient in Fig. 6.16B (lower panel). For a rough estimate of contributions from higher order superstructure spots, their intensities as found in the previous section (Sec. 6.2.5) are used. In particular, the investigated (01) and (21) peaks both had intensities of about $5 \%$ of the first order spots. Considering the $40 \%$ intensity loss of the superstructure peaks after laser excitation (Sec. 6.3.3) and comparable intensities for the primary graphene and polymer peaks, this roughly amounts to an overall intensity change of $3 \%$, similar to the intensity loss displayed in Fig. 6.16B.

Ultimately, the change in graphene spot intensity most likely originates from a combination of the above mentioned effects. However, the good comparableness of the delaydependent data with the superstructure spot intensity transient advocates a strong contribution from higher order PMMA diffraction peaks superimposed on those of graphene, while a pump-induced TEF effect mainly shows in the overall shift of the diffraction image.

\subsection{Physical picture and discussion}

The individually presented observations on the recorded PMMA superstructure dynamics are now combined into a connected physical picture.

The initial system can be described by strongly physisorbed, long PMMA chain molecules on the graphene substrate (Fig. 6.17A). Individual polymer strands crystallize in a folded-chain configuration with an inter-chain spacing of $d=4.26 \AA$. These strands form three different types of domains, which are orientationally linked to the substrate. Specifically, domains of polymer crystallites have an approximate diameter of $10 \mathrm{~nm}$ and cover about $50 \%$ of the graphene substrate in these regions. Different domain types are rotated by $60^{\circ}$ with respect to each other and exhibit a double periodicity in one direction in terms of the graphene unit cell.

Upon illumination by the few-ps laser pulse, energy is absorbed primarily within the graphene, while the PMMA is highly transmissive for the $800 \mathrm{~nm}$ wavelength of the pump (Fig. 6.17B). For an incoming laser fluence of $3 \mathrm{~mJ} / \mathrm{cm}^{2}$, the initial graphene temperature rises by about $535 \mathrm{~K}$, with the thermal equilibration of the graphene lattice occurring on the same time scale as the laser excitation [252]. 
Within about $40 \mathrm{ps}$, thermal energy is transferred from the graphene to the superstructure (Fig. 6.17C). This process leads to a slight spatial expansion of the crystallite's chain-to-chain distance of about $1 \%$ at a fluence level of $3 \mathrm{~mJ} / \mathrm{cm}^{2}$. For this fluence, the bilayer temperature reaches $165^{\circ} \mathrm{C}$, which is comparable to the melting temperature of bulk PMMA.

For higher fluences above the threshold level, the superstructure order is lost (Fig. 6.17D). Specifically, a deregistration of the polymer chains from the substrate on a 100 ps time scale is observed. The process is accompanied by a further temperature increase of the bilayer, which is derived qualitatively from the accelerated lattice expansion of the remaining crystalline components of about $5 \%$ within 130 ps.

For longer times up to $300 \mathrm{ps,}$ an amorphous state with expanded spatial components is formed. These components are peaked at a correlation length of about $5.7 \AA$ (Fig. 6.17E).

Finally, the initial crystalline state is largely (about $90 \%$ ) recovered after about $100 \mu \mathrm{s}$ (Fig. 6.17F). The very good reproducibility of the recrystallization process is ensured by the graphene, serving as a consistent structural template for the polymer chains.

The entirety of the observations, most notably the loss of order and substrate registration beyond a certain energy threshold, indicate a reversible phase transition between a crystalline and an amorphous phase of the superstructure.

A theory of melting in two dimensions has been developed by J. M. Kosterlitz and D. J. Thouless in the 1970s [22]. Based on this theory, D. R. Nelson and B. Halperin [253] have established a model for solid-liquid phase transition in a situation similar to the one experimentally investigated, namely of a two-dimensional film adsorbed to a periodic lattice. They distinguish between two general cases for the melting process of a commensurate crystalline overlayer phase.

1. A direct transition from a solid, crystalline phase, to a liquid phase at a melting temperature of $T_{m}$.

2. A two-step phase transition with an intermediate phase. In a first step, the solid adsorbate transitions to a so-called floating solid phase at a temperature $T_{m 1}$. This phase still remains basically crystalline, but has no more registration to the substrate. Instead, it can be understood as crystalline islands floating on the 


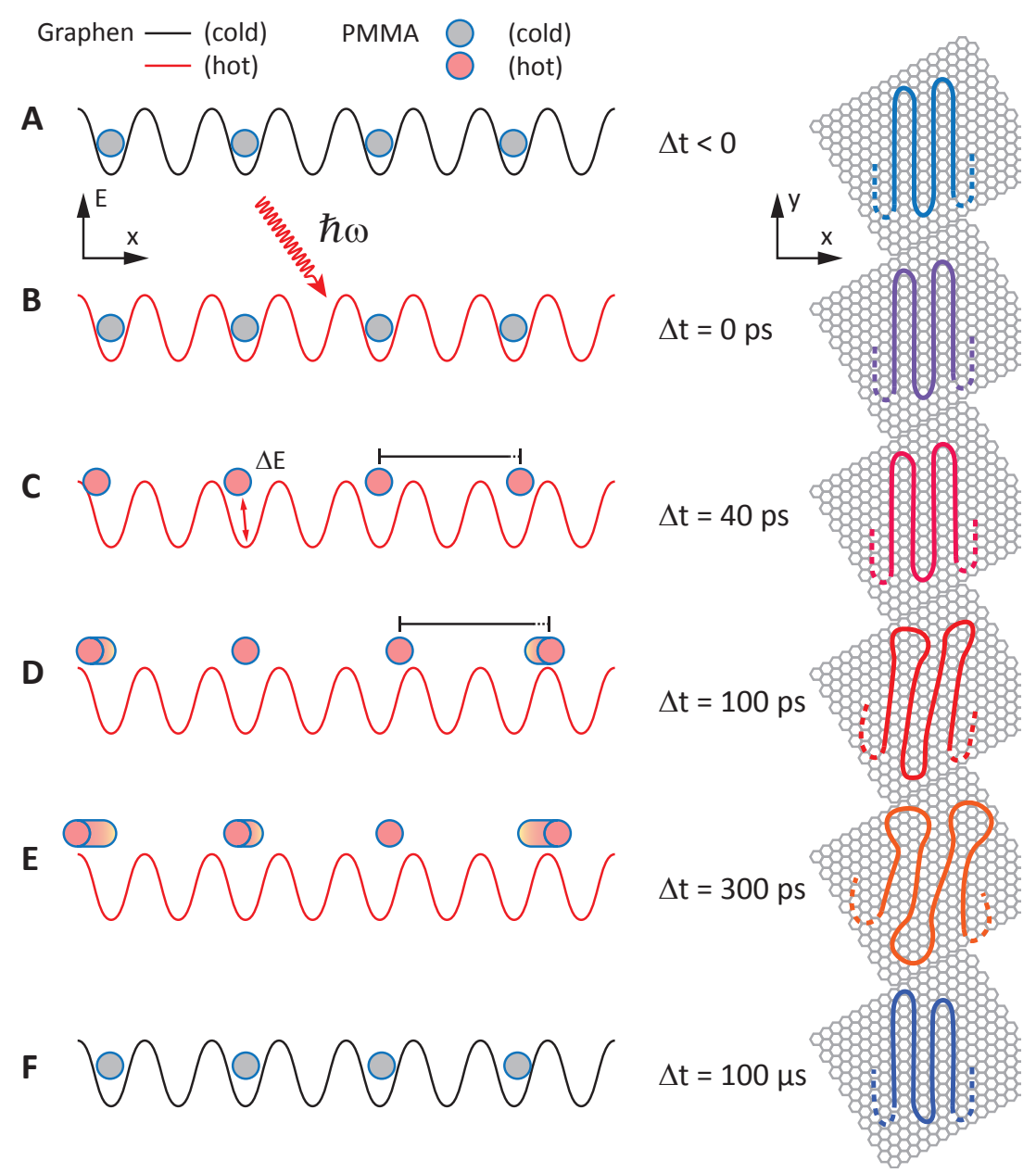

FiguRE 6.17: Sketch of a physical picture of the superstructure dynamics. Left images: Energy-space representation of corrugated graphene potential and PMMA strand cross-sections. Right images: Top-down view of PMMA conformation (red and blue) on graphene substrate (gray). Temperature of PMMA indicated by reddish (warmer) and bluish (colder) colors. A: Unpumped system. B: Graphene heating by light absorption (energy $\hbar \omega$ ). C: Energy transfer to the PMMA, spatial expansion. D: Loss of substrate registration and chain order, increased spatial expansion. E: Formation of amorphous components at low spatial frequencies. F: Cool down and near-complete recrystallization.

substrate. Above a temperature $T_{m 2}>T_{m 1}$, the floating solid loses its crystallinity completely and transitions to a liquid phase. For an experimental observation of this two-step process, see Ref. [254, 255].

The parameter, which ultimately decides for the specific pathway (1) or (2) in the solid-to-liquid transition is the fineness of the substrate lattice as a measure for the relative strength of graphene-adsorbate and intra-adsorbate coupling. In particular, a very fine substrate mesh relative to the superstructure lattice advocates the twostep process, while in a relatively coarse mesh, the periodic perturbations prevent the 
formation of a floating solid. Quantitatively, the criterion for the two-step process can be expressed as $c^{*} / b^{*}>\nu$, with $c^{*}$ the minimum common nonzero reciprocal lattice vector of substrate and superstructure, $b^{*}$ the minimum reciprocal lattice vector of the overlayer, and $\nu$ a constant depending on the Poisson ratio of the material. In the current case, $2.36<\nu<2.55$, for a typical Poisson ratio of PMMA between 0.35 and 0.40 . Following from this criterion, the observed phase transition of the PMMA superstructure in the present case would be of the first kind, hence a direct solid-liquid transition.

At this moment, the character of the transition cannot clearly be extracted from the available data: Since the rotation time of a single adsorbate domain on the substrate is large compared to the typical time scales in the experiment, a potential angular spread of the superstructure diffraction spots will be challenging to observe. However, the enhanced expansion of the superstructure lattice parameter as well as continuing temperature increase above the fluence threshold could be interpreted as the beginning formation of an incommensurate floating-solid phase. Nevertheless, it has to be taken into account that the data is integrated over a relatively large sample area, leading to a strongly averaged signal.

For a determination of the transition character, different approaches could be employed: For example, fluence-dependent measurements at very long delay times comparable to the characteristic domain rotation time could indicate a two-step process by a widening of the azimuthal superstructure spot profile. Complementary, atomistic molecular dynamics simulations of a polymer-graphene bilayer system as found in the experiment could give insight into the melting behavior on the molecular level.

More generally, the transition between the solid and the liquid phases could be explained, for example, by the creation of increasingly large looped chain segments with enhanced mobility. These regions at the border of the crystalline domains would not exhibit a crystalline order anymore (indicated in Fig. 6.17E). In this picture, the velocity given in Eq. 6.5 could be interpreted as the phase front velocity during the floating-solid-to-liquid transition.

Considering that phase front velocities are commonly of a similar order as the speed of sound of the material, e.g. about $200-500 \mathrm{~m} / \mathrm{s}$ for surface melting of $\operatorname{Si}(111)$ [256], which has a direction-depending speed of sound between $v_{s}(h k l)=5-8 \cdot 10^{3} \mathrm{~m} / \mathrm{s}[257]$, the found value of $v_{p h} \approx 6 \mathrm{~m} / \mathrm{s}$ seems comparably small. We therefore try to get an 
estimate on the order of magnitude of this velocity by means of the Frenkel-Wilson law [258]:

$$
v_{p h}=v_{0} \exp \left(-\frac{E_{a}}{k_{B} T}\right)\left(1-\exp \left(-\frac{L_{m}\left(T_{m}-T\right)}{k_{B} T_{m} T}\right)\right)
$$

In Eq. $6.10, v_{0}$ is a pre-exponential factor commonly of the order of the velocity of sound of the material, $E_{a}$ the activation energy for melting per atom, $k_{B} T$ the thermal energy at a given temperature $T$, and $T_{m}$ the melting temperature. Additionally, to apply this model, we need to momentarily assume that we have a well-defined latent heat $L_{m}$ per PMMA monomer. With $v_{0} \approx 2780 \mathrm{~m} / \mathrm{s}$ taken as the velocity of sound for PMMA, and $L_{m} \approx E_{a} \approx k_{B} T_{m}$ with $T_{m} \approx 430 \mathrm{~K}$ the melting temperature of bulk PMMA, this results in phase front velocity of $v_{p h} \approx 55 \mathrm{~m} / \mathrm{s}$. This value is still significantly larger than the experimentally obtained one, but of about the correct order of magnitude, supporting the idea of its interpretation as a phase front velocity. However, the polymer character has not been taken into account in this rough estimate and we used an upper-limit value for $v_{0}$. In this light, a more sophisticated description is needed to correctly extract a more precise value for $v_{p h}$ for this particular system.

\subsection{Summary}

In this chapter, the ultrafast dynamics of a polymer superstructure adsorbed on freestanding graphene have been investigated by the newly developed transmission ULEED setup.

The observed relaxation processes, including a loss of crystalline order and the formation of an amorphous phase, in combination with the clear threshold behavior have led to the conclusion that the superstructure is reversibly melted by intense laser excitation. Specifically, the large span of time constants connected to the various processes can be interpreted as a direct result of a hierarchical order of contributing coupling strengths. This includes the relatively weak registration of the polymer to the substrate on the one hand, and the strong polymer backbone, ultimately limiting the speed of the melting process, on the other hand. However, a direct correspondence between the involved coupling strengths and their respective influence on the superstructure melting necessitates further investigations. 
Similarly, the pathway of the two-dimensional melting process is not clearly resolved. In particular, there exist arguments for both types of melting, namely a direct solid-toliquid transition as well as a two-step process involving a floating solid phase without substrate registration.

Furthermore, it cannot be determined with certainty at the moment, if the enhanced expansion of the adsorbate lattice parameter is caused by a loss of crystallinity of the superstructure or if it is driving the melting process itself. Yet, with an observed chainto-chain distance at the lower end of the values reported for Langmuir-Blodgett films produced from PMMA, the second hypothesis seems more likely [110].

In conclusion, an atomistic molecular dynamics approach, based on the observations made in the experiment, would be extremely helpful to obtain a more detailed physical picture. Moreover, it can potentially help to understand open questions including the correspondence between the characteristic time constants and the involved coupling strengths, the observed discrepancy in time scale when compared to theoretical results for similar systems [259], as well as the nature of the phase transition itself. 



\section{Chapter 7}

\section{Conclusions}

\subsection{Summary}

The work performed in the framework of this thesis comprises two main points, namely the development of an ultrafast LEED setup, based on a nanometric pulsed electron source and its application to resolve the superstructure dynamics of an atomically thin polymer film adsorbed on free-standing graphene.

In the first part of the thesis, the properties of nonlinearly driven, needle-type photocathodes as electron sources for ultrafast low-energy imaging and diffraction experiments have been investigated in theoretical and experimental studies. Particular findings were:

- Nanometric photocathodes show exceptional beam properties, in particular low emittance, as well as high spatial coherence and brightness.

- Numerical FEM simulations on a tip-based electron source geometry confirmed a strong suppression of pulse broadening effects. Additionally, the optimal operation conditions for ultrahigh temporal resolution at low electron energies were determined.

- A prototype electron source was realized, displaying very good overall beam properties in terms of brightness, and coherence.

- Measurements performed on ultrathin films suspended on graphene displayed the electron source's long-term stability as well as its ultimate surface sensitivity down to a single atomic layer. 
- Experimental measurements demonstrated temporal resolutions of few picoseconds at electron energies comparable to classical LEED experiments.

The theoretical and experimental results attest to the outstanding performance of nanometric photocathodes within stroboscopically operated ultrafast experiments.

The applicability of the newly developed source has subsequently been demonstrated within an ultrafast LEED setup in transmission. Specifically, it was used to resolve the superstructure melting dynamics of an ultrathin bilayer system of atactic PMMA adsorbed on free-standing graphene, leading to the following observations:

- Individual polymer chains form orientationally linked, folded-chain superstructure configurations of double periodicity with respect to the graphene.

- This superstructure can be reversibly melted by intense laser irradiation, triggering a multitude of sequential relaxation processes.

- For the PMMA / graphene system, a thermal boundary resistance of $R_{k}=5.8$. $10^{-8} \mathrm{~m}^{2} \mathrm{~K} / \mathrm{W}$ was found from the measured energy transfer time across the bilayer interface of the order of $40 \mathrm{ps}$.

- A loss of crystallinity occurs within about 100 ps, paralleled by a further temperature rise as well as an continuous increase of the superstructure lattice parameter.

- An amorphous phase with pronounced low spatial frequency modes and characteristic, scattering-angle-dependent time constants between $150 \mathrm{ps}$ and $300 \mathrm{ps}$ is subsequently formed.

- Nearly complete recrystallization of the amorphous polymer happens within $100 \mu \mathrm{s}$.

The detailed account on the system's ultrafast relaxation from an extreme out-ofequilibrium state underline the feasibility of the presented approach, making ULEED a new and versatile tool in ultrafast surface and material science.

\subsection{Outlook}

In terms of the superstructure dynamics, these results present an interesting starting point for further theoretical investigations in terms of an atomistic molecular dynamics 
approach. To this end, a cooperation with the group of V. Harmandaris at the University of Crete, Greece, has been initiated. In view of the groups experience in the numerical investigation of graphene / polymer composite materials, an implementation of the system experimentally studied in this thesis should result in valuable insights. In particular, the effects of molecular length and substrate coverage, the correlation between coupling strengths and observed time scales, as well as the nature of the melting process itself are intriguing questions to be investigated.

Currently, first simulations on less complex sample systems, in particular united-atom simulations of a single polyethylene $(\mathrm{PE})$ chain close to the graphene substrate, are performed at different temperatures. The chosen potential is of the van der Waals type, in particular a semi-empirical formulation of dispersion and London interactions. Preliminary observations of the simulations are:

- The PE nearly immediately adsorbs to the graphene substrate on a few-ns time scale. The initial three-dimensional PE configuration with respect to the graphene is shown in Fig. 7.1A, its projection on the substrate plane in Fig. 7.1B.

- For low and intermediate temperatures (300 K and $350 \mathrm{~K}$ ), a folded chain configuration is adopted on a time scale of few tens of nanoseconds, depending on the temperature of the system (Fig. 7.1C and D, respectively). For higher temperatures $(T>400 \mathrm{~K})$, this conformation is lost (Fig. 7.1E).
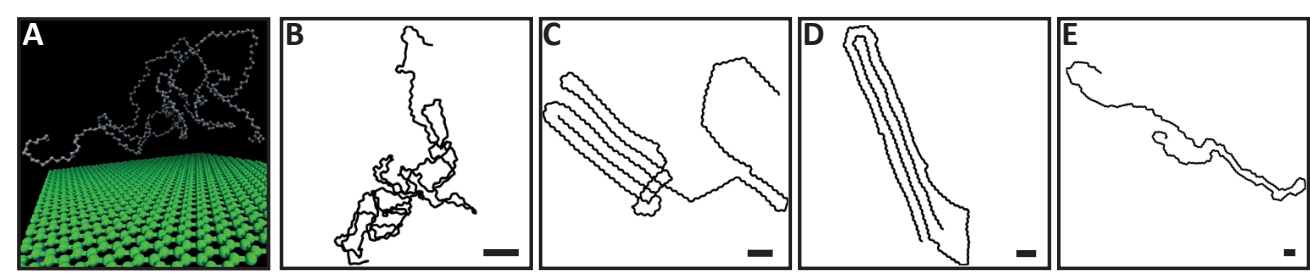

FiguRE 7.1: Exemplary PE conformations from preliminary molecular dynamics simulation on graphene. A, B: Initial PE conformation in 3D (A) and projected to the graphene plane (B). C: Folded-chain conformation at $300 \mathrm{~K}$ after 40 ns. D: Foldedchain configuration at elevated temperatures, different orientation to substrate. E: PE at $450 \mathrm{~K}$ after $40 \mathrm{~ns}$ without folded-chain character. Bar denotes length scale of $1 \mathrm{~nm}$.

- This behavior is mirrored by the evolution of the dihedral angle distribution, specifically by a reduction of gauche configurations with respect to the number of trans configurations in the chain (Fig. 7.2C). This is very close to the distribution expected for a crystalline state. 
- Single PE strands are orientationally linked to the substrate at lower temperatures (Fig. 7.1C, Fig. 7.2B), but loose this linkage at intermediate temperatures, while crystallinity is conserved (Fig. 7.1D).

- The crystal chain conformation displays a double periodicity with respect to the graphene, as can be seen in Fig. 7.2A and B.

- The preferred segment length is temperature dependent. It increases until after the point when substrate registration is lost (Fig. 7.1C) and is subsequently reduced in the amorphous state (Fig. 7.1E). An exemplary temporal evolution is given in Fig. 7.2D. Here, the segment length distribution is weighted by the number of monomers per segment. For $300 \mathrm{~K}$, a preferred fold length of $9-10 \mathrm{~nm}$ is found, for $350 \mathrm{~K}$ this increases to $15-20 \mathrm{~nm}$.
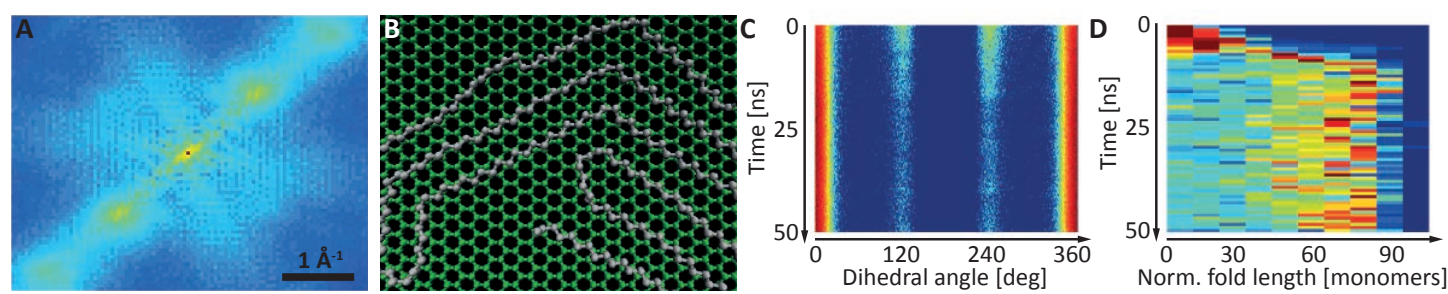

FiguRE 7.2: A: Fourier transformation of conformation shown in Fig. 7.1C. B: Orientation of $\mathrm{PE}$ on graphene substrate for A. C: Dihedral angle distribution. D: Foldedchain segment length distribution, normalized to the number of monomers per fold.

These preliminary results are in very good agreement with the experimental observations on the PMMA / graphene bilayer, e.g., concerning the polymer conformation, its periodicity, orientation and segment length. Further simulations are going to be performed in the near future, including the analysis of a system excited to a state far out-of-equilibrium.

Furthermore, it is planned to experimentally study different types of polymers, for example PE or isotactic PMMA, which has been observed to crystallize in a helical structure [110]. This would allow for the analysis of correlations within single chains, and hence support a differentiation of the respective dynamics from those based on inter-chain interaction. Additionally, the investigation of overlayer lattices with different periodicity might shed some light into the underlying pathway of melting, as previously discussed.

On the technical side, ULEED is going to be further improved, including the possibility of sample heating, the use of a low-noise, high resolution camera system, and measures 
to further increase the temporal resolution of the current system (Sec. 5.4.2). With the improvement of the temporal resolution of ULEED, for example, new systems featuring smaller time scales will be accessible. This may include significantly smaller organic molecules, down to dimers and monomers, eventually allowing for a correlation between the detailed microscopic structures and their macroscopically observed dynamical properties.

Apart from thin films and two-dimensional crystals, the study of dynamics on bulk surfaces of course offers a tremendous amount of intriguing systems, such as adsorbate dynamics on metal surfaces for the study of heterogeneous reactions in femtosecond surface chemistry [260] or surface charge density waves [29]. This will necessitate an implementation of ULEED in the normal-incidence backscattering geometry, as commonly used in LEED setups. The resulting system will potentially be capable of employing the full analytical power of static LEED systems, while additionally allowing access to dynamical properties on ultrafast time scales.
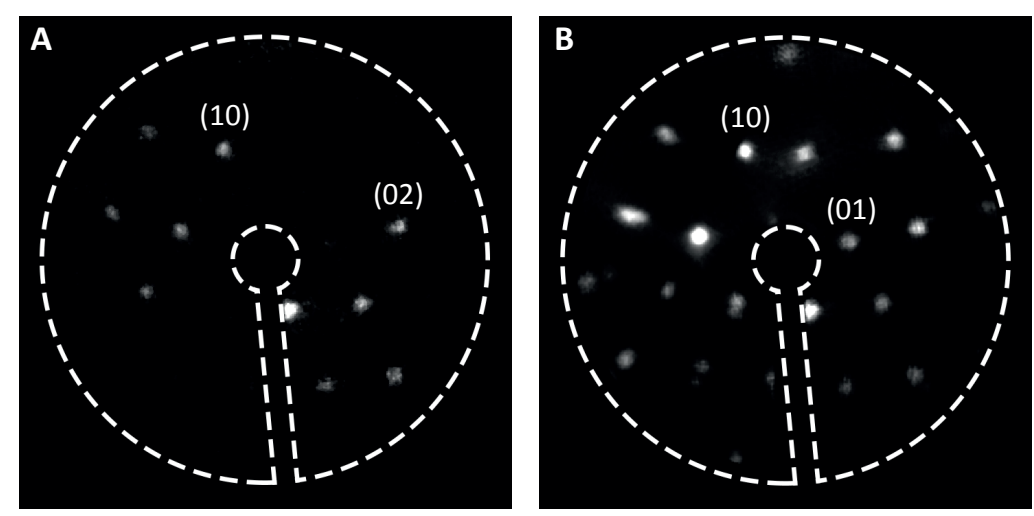

FIGURE 7.3: Ultrafast LEED diffraction images of a single crystalline sapphire (0001) surface in back-reflection geometry. Angle of incidence: $45^{\circ}$. Electron energy: $380 \mathrm{eV}$ (A) and between $335 \mathrm{eV}$ and $435 \mathrm{eV}(\mathbf{B})$. Distortion stems from planar detector employed.

The current setup is only partially usable in back-reflection geometry with the minimal angle of incidence limited by the relatively large electron gun (outer diameter $6 \mathrm{~cm}$ ). However, measurements of a sapphire surface at an angle of incidence of about $45^{\circ}$ have been performed to demonstrate the general feasibility of operation in back-reflection (Fig. 7.3).

There is no fundamental constraint on developing ultrafast LEED for the classical normal incidence geometry based on the methods provided within this work (Fig. 7.4A). In contrast, it is expected from numerical simulations that the experimental parameters 
such as pulse duration and electron beam coherence will strongly benefit from a smaller gun.

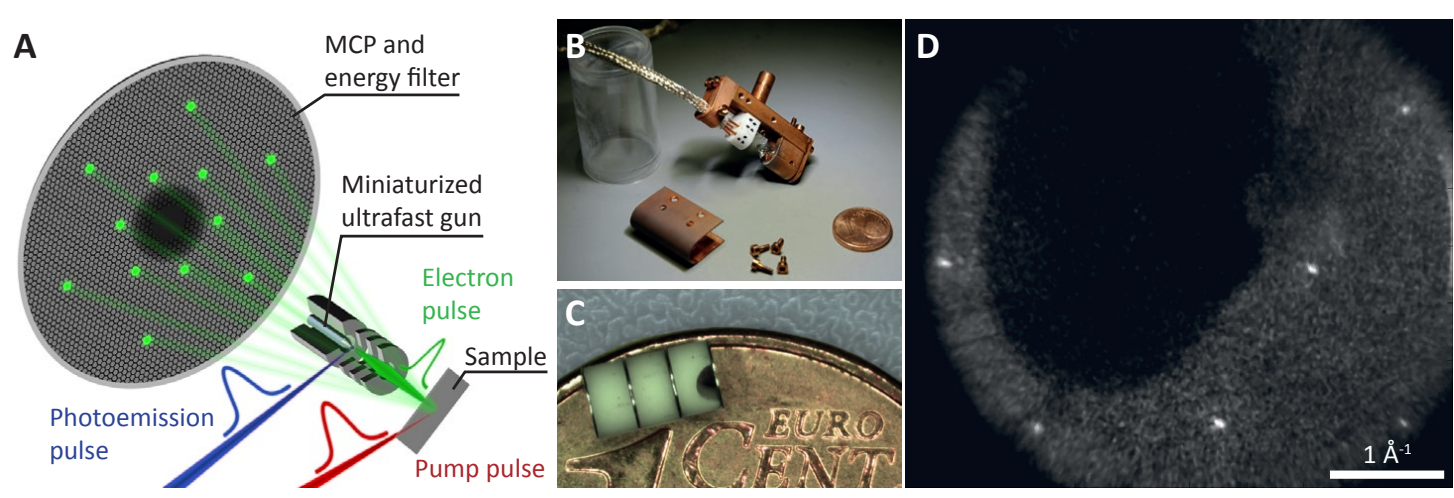

FiguRE 7.4: Ultrafast LEED in normal-incidence back-reflection geometry. A: Sketch of the experimental setup featuring a miniaturized tip-based ultrafast electron gun. B, C: Photograph of the electron source (B) and electrostatic lens system (C). D: First diffraction image of single crystalline sapphire (0001) recorded with the new electron source. Dark center area stems from shadowing of the electron gun. Electron energy:

$150 \mathrm{eV}$. Integration time: $120 \mathrm{s.}$ [261]

On these grounds, first steps in this direction are currently undertaken by the development of a significantly smaller electron source (Fig. 7.4B and C, outer diameter 7 $\mathrm{mm})$. Even though this source is still under development, first diffraction images from a sapphire surface within a normal-incidence geometry have been recorded Fig. 7.4D). A temporal characterization of the electron pulses as well as first time-resolved diffraction experiments are under way.

In comparison with other ultrafast electron-based methods, the present experimental setup features a relatively simple and low-cost approach, e.g., by the utilization of readily available components for regular ultrafast optics experiments. It is hoped that this will make it accessible to a broad scientific community in the near future.

More generally, tip-based electron sources are not only interesting in terms of low-energy applications such as time-resolved LEED. Instead, they are also promising candidates to further advance existing ultrafast imaging and diffraction techniques at higher energies, e.g., in time-resolved RHEED and TEM experiments. To this end, an ultrafast TEM based on a similar needle emitter as employed in ULEED, is currently under development in our laboratories (Fig. 7.5). The use of a nanometric photocathode has been extensively investigated in simulations and has been seen to result in excellent beam properties, capable to further increase the coherence and temporal resolution compared to current ultrafast implementations of TEM [182]. 

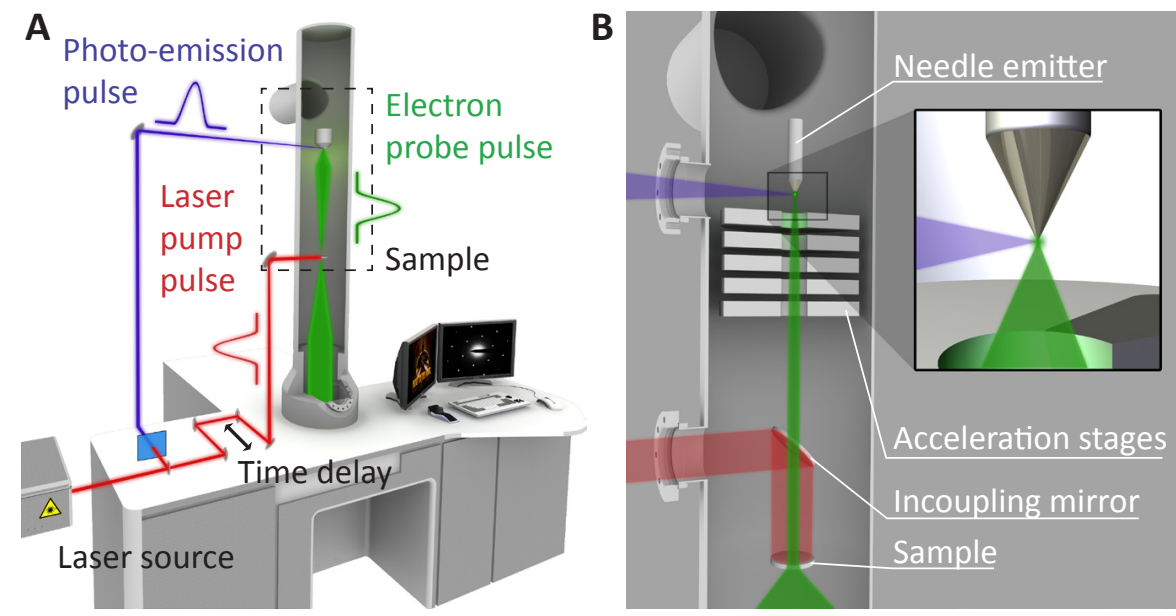

Figure 7.5: Schematic of an ultrafast TEM based on a nanometric tip-based photocathode. A: Laser-pump / electron-probe scheme of the TEM. Dashed black rectangle denotes area magnified in B. B: Inside view of an ultrafast TEM with laser driven needle emitter gun.

To conclude, ULEED and its future developments carry the potential to be the basis of investigating previously inaccessible surface and thin film atomic structural dynamics on their intrinsic ultrafast time scales. 



\section{Appendix A}

\section{Methods and Calculations}

\section{A.1 Analytical model for pulse durations}

The spatio-temporal broadening of electron pulses has three main contributors [165]: A finite width of the initial electron energy spectrum, path length differences on the way to the anode and Coulomb repulsion within the electron bunch. With pulse durations of the order of below 100 femtoseconds, the interaction time of the emitter with the photoemission laser pulse is usually a negligible factor.

Broadening from inter-electron interaction can also be discarded, when operating in a regime of few electrons per pulse [50]. Path length differences, however, arise from the specific gun geometry and lens system employed and will not be taken into account in this analytical model for the sake of simplicity. These considerations leave the initial electron energy spread as the sole broadening factor, which is in agreement with the results obtained from the finite element simulations at least for intermediate and high values of $\Delta E$ (Chap. 3).

The following calculation is based on the model presented by S. Schweda in Ref. [191], but implements further approximations to obtain a simple expression for the pulse duration $\tau_{\text {pulse }}$ as a function of the initial energy spread $\Delta E$.

First, the time of flight of an electron within a plate capacitor geometry (Fig. A.1A) for a given distance $d$ between anode and cathode and an acceleration voltage $U$ is analytically determined. 
A

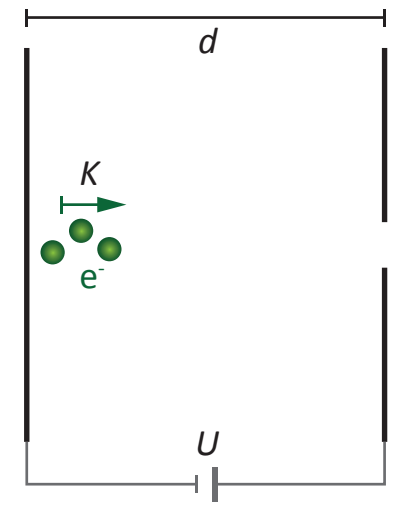

B

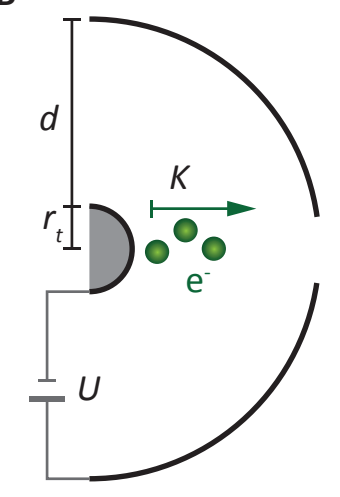

Figure A.1: Sketches of plate capacitor $(\mathbf{A})$ and needle emitter $(\mathbf{B})$ geometries. Variables: $d$ : distance between emission site and anode; $U$ : accelerating voltage; $K$ : Force acting on electron; $r_{t}$ : tip apex radius of curvature.

The force acting upon a single electron within the plate capacitor may be written as

$$
K=\frac{e U}{d}=m_{e} \ddot{r}
$$

where $e$ and $m_{e}$ are the charge and mass of the electron, respectively. Single integration leads to

$$
\dot{r}=\frac{e U}{m_{e} d} t+v_{0}
$$

with the initial velocity given by $v_{0}=\sqrt{2 E / m_{e}}$, and $E$ being the initial electron energy. The time of flight $T(E)$ can then be written as

$$
T(E)=\frac{\sqrt{2 m_{e}} \cdot d}{e U}\left(\dot{r}(t=T)-v_{0}\right)=\frac{\sqrt{2 m_{e}} \cdot d}{e U}(\sqrt{e U+E}+\sqrt{E}) .
$$

The pulse duration for a given energy spread $\Delta E$ is then simply the difference

$$
\tau_{\text {pulse }}=T(E+\Delta E)-T(E) .
$$

In the case of the tip geometry (Fig. A.1B), the velocity is again given by

$$
v(t)=\sqrt{2(E+e \Phi) / m_{e}},
$$

with $\Phi=\Phi(r)$ a radially dependent potential. For simplicity, we chose $\Phi(r)$ to be of the form:

$$
\Phi(r)=\frac{a}{r}+b
$$


The constants a and b can be determined by the boundary conditions $\Phi\left(r_{t}\right)=0$ and $\Phi\left(r_{t}+d\right)=U$, where $r_{t}$ is the tip radius. This form emphasizes the potential decay stronger in comparison with the logarithmic potential given in Ref. [161], but poses still an adequate approximation. Additionally, it has the advantage of delivering an analytical result for the pulse duration. For a more detailed account on the $r$-dependence of the potential of a nanometric tip, see Ref. [174].

For simplicity and a nanometer sized tip as well as macroscopic propagation distances, we can further approximate $r_{t}+d \approx d$, so that the potential now reads

$$
\Phi(r)=U-\frac{U r_{t}}{t}
$$

By separation of the variables, the time of flight can then be written in terms of the following integral:

$$
\int_{0}^{T} d t=T(E) \approx \int_{r_{t}}^{T} \frac{d r}{\sqrt{\frac{2(E+e \Phi(r))}{m_{e}}}} .
$$

This integral can be analytically solved [262] by rewriting it in the form

$$
\int \sqrt{\frac{r}{B_{1} r+B_{2}}} d r
$$

with $B_{1}=B_{1}(E)=2(E+e U) / m_{e}$ and $B_{2}=-2 r_{t} e U / m_{e}$, leading to

$$
T(E) \approx \frac{1}{B_{1}} \sqrt{r\left(B_{1} r+B_{2}\right)}-\frac{B_{2}}{B_{1}^{3 / 2}} \log \left(\sqrt{B_{1} r}+\sqrt{B_{1} r+B_{2}}\right) .
$$

The pulse broadening is then again calculated by the difference as given in Eq. A.4. With $B_{2} \ll B_{1} r$ and the second term in Eq. A.10 also more than three orders of magnitude smaller than the first within a typical tip geometry, the pulse duration can be written as

$$
\tau_{\text {pulse }}=\sqrt{\frac{m_{e}}{2}} d\left(\frac{1}{\sqrt{e U+E_{0}+\Delta E}}-\frac{1}{\sqrt{e U+E_{0}}}\right) .
$$

In this approximation, the tip radius is not included anymore, which coincides with the results from the simulation, namely that the radius of the tip apex does not strongly influence the pulse duration (Sec. 4.4). A more sophisticated model of the propagation dynamics of an ultrashort electron pulse can be found in Ref. [165].

For a rough comparison with the results from the FEM calculations, Fig. A.2 displays the 
computed pulse durations for a propagation distance of $d=12 \mathrm{~mm}$ in the tip geometry for both cases, $\alpha=0^{\circ}$, and $\alpha=10^{\circ}$. For low to intermediate $\Delta E$, the analytical approach agrees well with the FEM simulations with electrons only along the optical axis, but increasingly overestimates the pulse duration at higher energetic spreads.

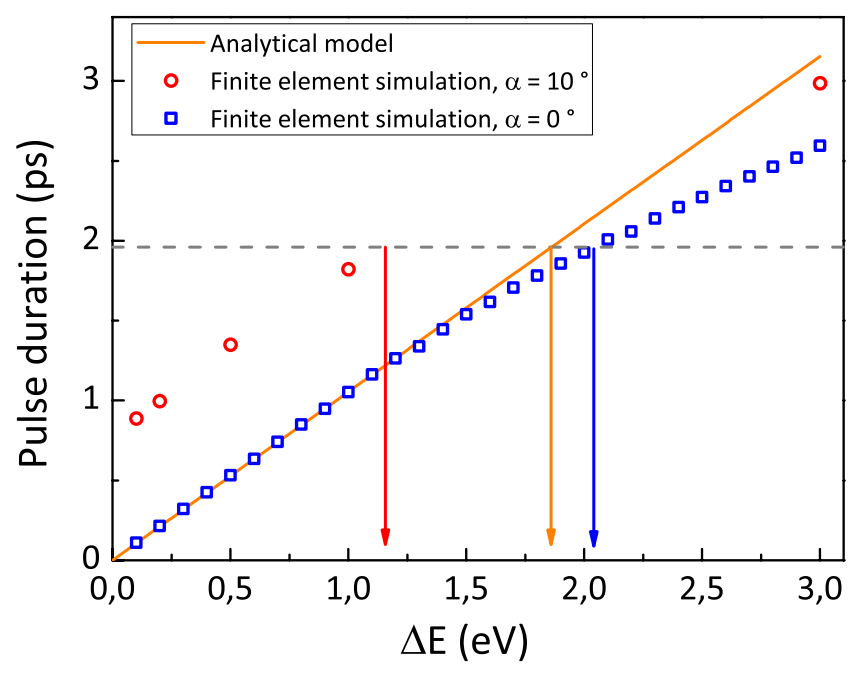

Figure A.2: Comparison of analytical model with results from the finite element simulations for the pulse duration at a given energy spread $\Delta E$. Electron energy: $450 \mathrm{eV}$. The dashed line denotes the pulse duration found in the experiment at the same energy. The red, blue and orange arrows show the resulting energy spread for to the experimental pulse duration within the FEM $\left(1.13 \mathrm{eV}\right.$ for $\alpha=0^{\circ}, 2.03 \mathrm{eV}$ for $\left.\alpha=10^{\circ}\right)$ and the analytical $(1.86 \mathrm{eV})$ model, respectively.

When compared to the experimentally obtained pulse duration at the same energy of $450 \mathrm{eV}$ (gray dashed line), the energetic spread can be estimated for both the FEM (red arrow) and the analytical model (orange arrow). While the FEM for $\alpha=10^{\circ}$ yields a value of about $1 \mathrm{eV}$ in good agreement with the experimentally obtained value, the analytical model as well as the simulations with $\alpha=0^{\circ}$ yield a larger spread of around $2 \mathrm{eV}$. Interestingly, the inclusion of electrons not on the optical axis leads to a more or less constant offset in the temporal resolution. This could potentially help to quickly estimate the pulse duration a given energetic spread and to indirectly measure the actual emission cone of the electron source.

\section{A.2 The electron inelastic mean-free-path}

The universal curve for the electron IMFP for energies between $1 \mathrm{eV}$ and $10 \mathrm{keV}$ consists of two branches with different functional dependency on the electron energy: between 
$1 \mathrm{eV}$ and about $20 \mathrm{eV}$, it displays a proportionality to $E^{-2}$, for energies above $100 \mathrm{eV}$, it is proportional to $E^{1 / 2}$.

Qualitatively, this behavior can be understood in the following way: In the low-energy limit, the functional behavior is dominated by excitation of electron-hole pairs [263]. Specifically, an electron of energy $\mathrm{E}$ has a lifetime $(\tau \propto \mathrm{IMPF})$, which is inversely proportional to a) the number of excitable conduction electrons $(\propto E)$ as well as b) the number of unoccupied states to transfer to $(\propto E)$ [264]. Hence, in the low-energy limit, the functional dependence is IMFP $\propto E^{-2}$. Generally, the IMFP is slightly enhanced for organic molecules [265]. For an more in-depth theoretical treatment (also in the case of higher energy electrons), see Ref. [266].

With increasing energy, ionization of core electrons and plasmonic excitation also become possible, leading to a further reduction of the IMPF. For higher energies of about $100 \mathrm{eV}$ and above, one can approximate the inelastic scattering cross-section ${ }^{1}$ as being proportional to the interaction time of the passing electron. With this interaction time inversely proportional to the velocity of the electron and hence to $E^{-1 / 2}$, we get IMPF $\propto E^{1 / 2}$.

The curves in Fig. 2.1B are calculated using the best-fit equations in the conclusions of [63] for elements with the appropriate values for graphite.

\section{A.3 Finite element method}

The FEM is a numerical procedure to solve partial differential equations. In particular, FEMs are used to find an approximate solution for a simplified mathematical model [267]. They are used in a wide variety of engineering problems and have the advantages of being able to handle complex geometries and boundary conditions. Major drawbacks are the production of only approximate solutions and, as common in numerical methods, the lack of a general closed-form solution, which would allow for a parametric analysis of the problem.

The general concept of FEMs is based on a discretization of the simulation volume in a number of finite-sized sub-volumes (the so-called mesh), which are itself described by a

\footnotetext{
${ }^{1}$ Since the elastic scattering coefficient of electrons does usually not exceed a few percent, it will be ignored in the following argumentation [264].
} 
number of parameters. Ideally, the solution obtained from the FEM converges against a fixed value for an increasingly fine discretization. Since the computational effort increases with the number of elements within the simulation volume, it is important for many applications to develop "smart" meshes. In context of the simulations performed for the tip-geometry, this was achieved by defining different spatial resolutions for various areas, resulting, e.g., in a very fine discretization at the tip apex and larger elements for non-critical regions. The mesh generation was performed with the help of GMSH after the initial geometry construction with MATLAB. To complete the discretization, a basis for solutions with constant boundary values is computed using cubic finite elements on triangles.

The governing equation for the electrostatic boundary problem is Laplace's equation, commonly written as $\Delta U=0$, with $U$ the electrostatic potential. When cylindrical coordinates $(r, \varphi, z)$ are employed, the problem reads

$$
\Delta U=\frac{1}{r} \frac{\partial}{\partial r}\left(r \frac{\partial U}{\partial r}\right)+\frac{1}{r^{2}} \frac{\partial^{2} U}{\partial \varphi^{2}}+\frac{\partial^{2} U}{\partial z^{2}}=0
$$

Since the problem is cylindrically symmetric, the central term of Eq. A.12 is zero. Specifically, two types of boundary conditions are used, namely Dirichlet and Neumann boundary conditions. For the former, the value of the potential $U$ is fixed, thus Dirichlet boundary conditions apply to the lens elements and the tip. In contrast, Neumann boundary conditions are fulfilled, whenever the gradient of the potential with respect to the surface vanishes, and are employed to define the remaining volume surface.

A Runge-Kutta algorithm is used to numerically find the solution to the set of partial differential equations derived from Eq. A.12. In particular, the classical equation of motion for individual electrons with their initial conditions is solved. In the simulations presented in this work, the trajectories of about 9000 electrons have been simulated to obtain a single data point. The resulting temporal and radial spread are then given in terms of the FWHM of the respective distribution.

Apart from the initial mesh generation, all computations are performed within the MATLAB programming environment. For an estimate of the accuracy of the software, part of the results are reproduced in the COMSOL Multiphysics 4.3 package $^{2}$ and found to agree well.

\footnotetext{
${ }^{2}$ http://www.comsol.com/comsol-multiphysics
} 


\section{A.4 Data normalization and fitting}

All time-resolved measurements of the diffraction intensity data presented in this work are normalized to the recorded intensity at negative delay times $\Delta t=t-t_{0}<0$ with $t_{0}$ the temporal overlap between pump and probe pulses at the sample position. For measurements without temporal resolution, including the fluence-dependency, the diffraction intensity is normalized with respect to the unpumped sample. By taking into account only the relative intensity change, it is made sure that the effect of sample degradation is minimized.

Furthermore, the sample degeneration is simultaneously controlled by an alternating recording of the pumped and unpumped sample for each data point. Several of the figures in this work show fitted curves in addition to the time-resolved diffraction data. In particular, the temporal evolution of the diffraction intensity $I(t)$ is fitted by means of a single exponential $[149,268]$ :

$$
I(t)=\Theta\left(t-t_{0}\right) A \exp \left(-\frac{t-t_{0}}{\tau}\right)+I_{0}
$$

where $\Theta\left(t-t_{0}\right)$ is the Heaviside function to allow for fitting the intensity at negative delay times $I\left(t-t_{0}<0\right)=I_{0}$ and $A$ is the amplitude of the recorded intensity change. The temporal overlap between electron probe and laser pump pulse is determined prior to each measurement by means of the described TEF effect (Sec. 5.3.2) and is not used as a free parameter in the fitting process.

\section{A.5 Separation of diffraction spot and disc contributions}

In order to evaluate the intensity evolution of the adsorbate diffraction spots and the amorphous disc isolated from each other, the different contributions are separated by the following approach: Firstly, a radial averaging (Fig. A.3A) is performed for only the areas around (in between) the spots as shown in Fig. A.3B (C), while the intermediate regions (gray shaded areas) and the central beam block (black) are ignored. The angular selection is thereby performed manually within MATLAB. 

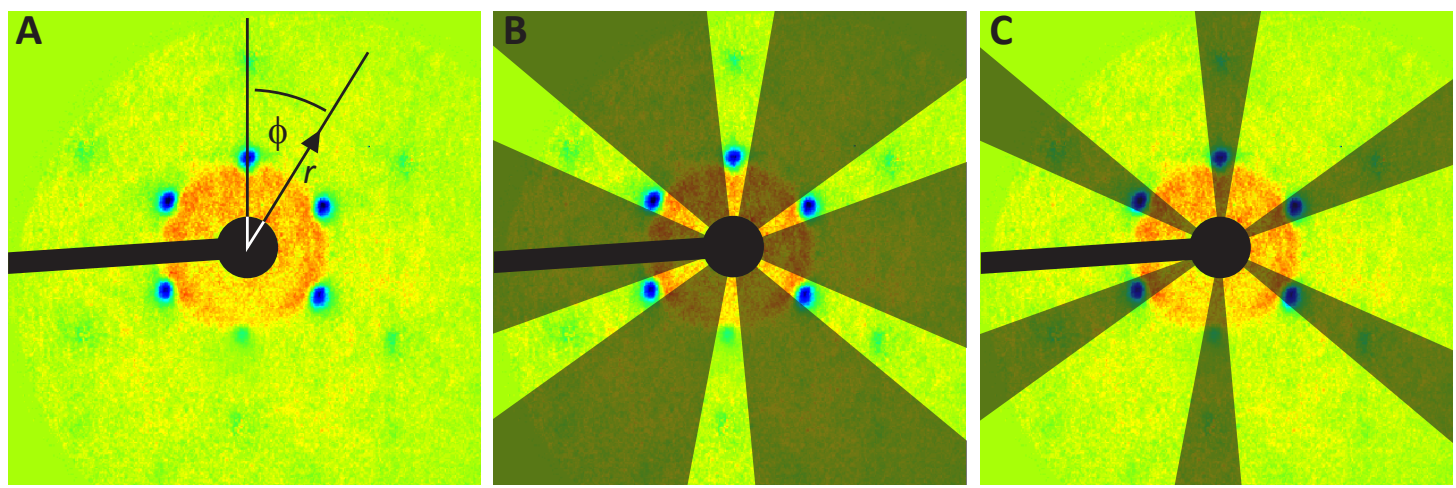

FiguRE A.3: Differentiation routine of isotropic and six-fold symmetric components of the diffraction pattern. Fluence: $6 \mathrm{~mJ} / \mathrm{cm}^{2}$ A: Difference image of a diffraction pattern recorded at large positive delay with respect to the same image taken at negative delays. Coordinate system indicated for angular averaging around the center of the pattern. B: The isotropic signal (gray shaded area) as well as the central beam stop (black) are ignored during the averaging process, leaving only the six-fold symmetric contributions.

C: The same as in B, but for the isotropic contribution.

Secondly, the so obtained isotropic contribution (Fig. A.3C) is subtracted from the signal acquired when averaging over the regions denoted in Fig. A.3B, leaving only the intensity change in the adsorbate peaks.

\section{A.6 Reproducibility of the measurements}

The performance of measurements on a sample system, which is as sensitive to degradation as the bilayer system presented in this work, is a challenge. To nevertheless maintain a good reproducibility of the experimentally obtained results, while taking the mentioned degradation of the probed system into consideration, the following steps are undertaken.

Firstly, all time-dependent measurements are recorded consecutively over multiple measurement runs ("loops"), and secondly, each data point / diffraction image is recorded between 10 and 30 times per run. We also performed measurements with a randomized order of delay points - to conform that cumulative effects for time dependent behavior have been avoided - and obtained the same result. However, in view of the limited exposure time for each sample, we decided to go through the delay points in chronological order to avoid an additional degradation of the sample during extended delay stage positioning. Furthermore, it is apparently of importance for the recording of high quality 
data to maintain constant recrystallization times for the polymer crystallites between pumping, again by avoiding randomly chosen delay points.

Figure A.4 displays the temporal evolution of the PMMA superstructure spot intensity decrease resolved for the four individually recorded measurement runs (red circles). For better comparison, the averaged data points (gray squares) as well as their exponential fit (blue dashed line) are also shown. The individual time constants for each plot are determined by using the same settings as in the case of the averaged data with only the decay time as a free parameter.
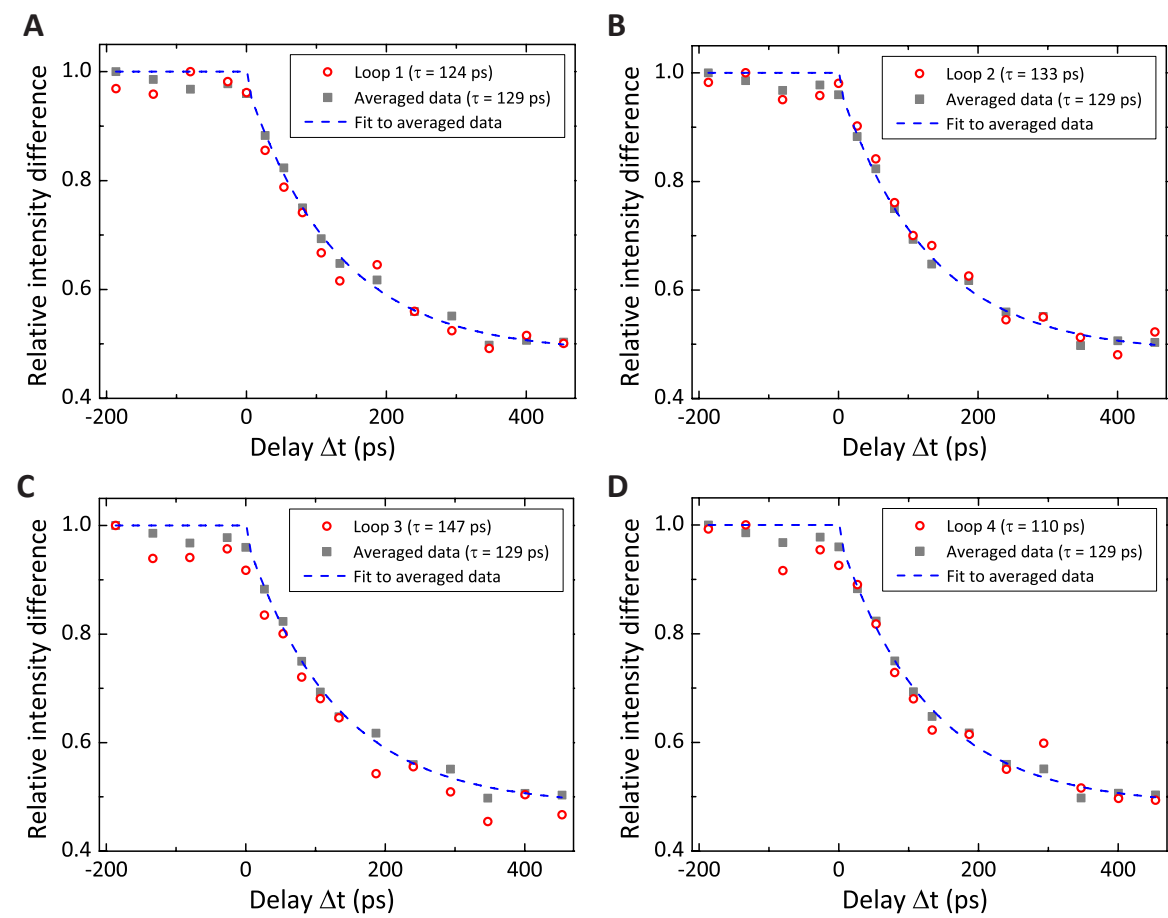

Figure A.4: Loop-resolved superstructure dynamics. Electron energy: $450 \mathrm{eV}$. Fluence: $7.1 \mathrm{~mJ} / \mathrm{cm}^{2}$. Diffraction images per point per loop: 15 . The graphs display the relative intensity difference of the superstructure diffraction peaks consecutively recorded four times $(\mathbf{A}-\mathbf{D})$ in comparison with the averaged values (gray squares) and their exponential fit (blue dashed line).

The amplitude of the intensity loss, the time-zero as well as the individual time constants agree well with the averaged data, leading to an overall decay time of 129 ps with a standard deviation of $14 \mathrm{ps}$. 



\section{Appendix B}

\section{Sample characterization}

\section{B.1 Graphene characterization}

There are several methods to determine the quality as well as the single layer character of the resulting graphene, including AFM, SEM, TEM, optical microscopy ${ }^{1}$ and Raman spectroscopy [71, 269]. For the samples used in this work, Raman spectroscopy is performed either directly on the $\mathrm{Cu}$ substrate or after transfer of the graphene to $\mathrm{SiO}_{2}$ wafers. In comparison with the other methods, Raman spectroscopy has the advantages, that it is not only giving a clear fingerprint of the graphene in terms of number of layers and single crystallinity, but it is also relatively simple, quick, non-destructive, and substrate independent [270].

Figure B.1 shows Raman measurements of single (Fig. B.1A [271] and Fig. B.1B) and multi-layer graphene (Fig. B.1B). The most prominent features are the G peak (around $1580 \mathrm{~cm}^{-1}$ ) and 2D peak ${ }^{2}$ (around $2700 \mathrm{~cm}^{-1}$ ). Whereas the $\mathrm{G}$ peak is scaling directly with the number of the graphene layer(s), the intensity of the 2D peak remains mainly unaffected by thickness variations $[269,270]$. Instead, the 2D peak stems from the second order of zone-boundary phonons (at around $1350 \mathrm{~cm}^{-1}$ ) [272].

Apart from the intensity relation between the $\mathrm{G}$ and 2D peaks the occurrence of single layer graphene can be determined from the shape of the 2D peak. Fig. B.1B, lower

\footnotetext{
${ }^{1}$ Single layers of graphene are visible under the optical microscope when deposited on oxidized $\mathrm{Si}$ substrates with very defined thickness. In this case, single carbon layers can be distinguished by slightly changing the optical path length of the transmitted light in comparison to the empty substrate.

${ }^{2}$ The $2 \mathrm{D}$ peak has been historically named $\mathrm{G}^{\prime}$.
} 
A

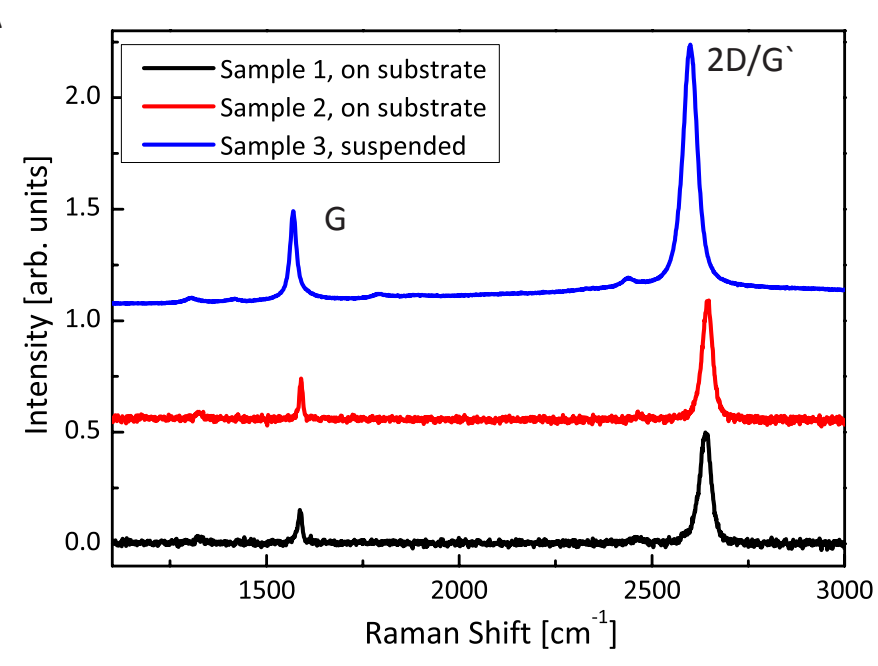

B

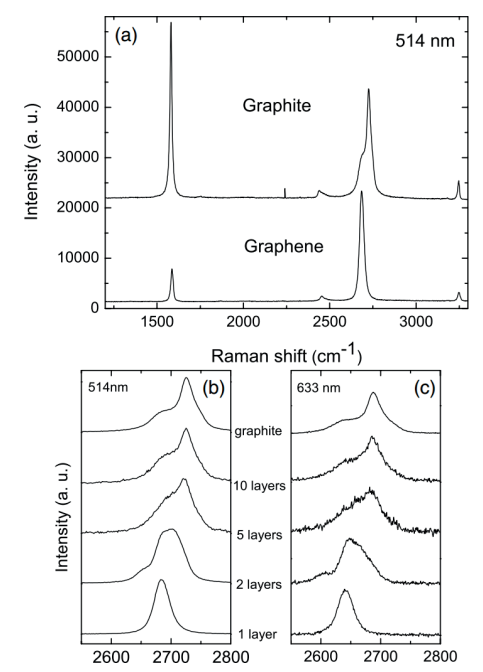

Figure B.1: Raman spectra of graphene samples showing $G$ and 2D peaks. A: Raman spectra taken from three graphene samples, two of them on a $\mathrm{SiO}_{2}$ substrate (red and black curves), one suspended (blue line). The weak $\mathrm{G}$ peak and the symmetric shape of the $2 \mathrm{D}$ peak indicate monolayer graphene. Excitation wavelength: $633 \mathrm{~nm}$. Laser beam spot size: $5 \mu \mathrm{m}$. Integration time: $20 \mathrm{~s}$. B: Reference spectra of single and multi-layer graphene as well as graphite taken from Ref. [269]. Excitation wavelengths: $514 \mathrm{~nm}$

(b) and $633 \mathrm{~nm}(\mathrm{c})$.

panel, illustrate the changing of the 2D peak shape from single to multi-layer graphene / graphite.

When taking the Raman signal from free-standing graphene, a small contribution from PMMA at around $2900 \mathrm{~cm}^{-1}$ can sometimes be observed Fig. B.2) in agreement with the PMMA signal as reported in the literature [273]. However, this feature is not detected in all sample areas and therefore likely due to a locally thicker PMMA film. For comparison, the PMMA film thickness in Ref. [273] was about $200 \mathrm{~nm}$.

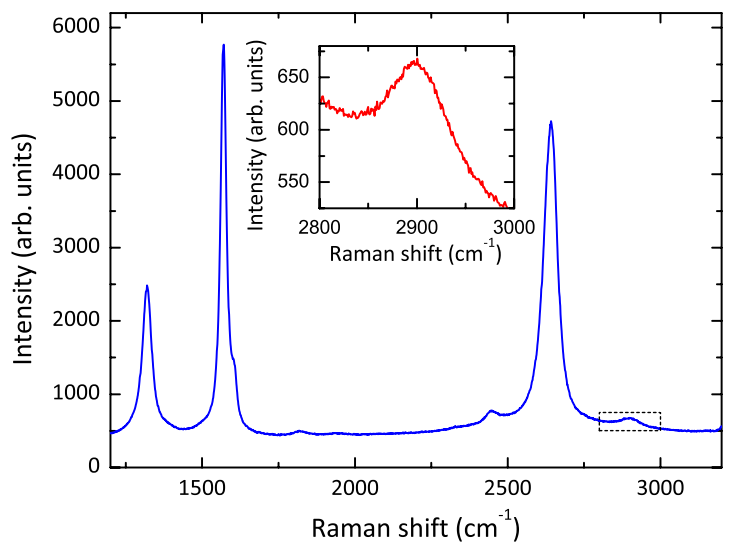

FiguRE B.2: Raman signal from bilayer sample with PMMA contribution. Inset: Magnified area denoted by dashed rectangle. 


\section{B.2 Formation time of the folded-chain crystallites}

We find that the diffraction signal of the polymer superstructure close to the central beam stop depends critically on the drying time. For a qualitative analysis, two samples ("1" and "2" denoting different positions on the sample) with varied drying times are compared in Fig. B.3. Both of them have been prepared in the same sample preparation run under identical conditions. The sample shown in A has been analyzed directly after etching and washing, whereas sample B was given $24 \mathrm{~h}$ to dry under atmospheric conditions.
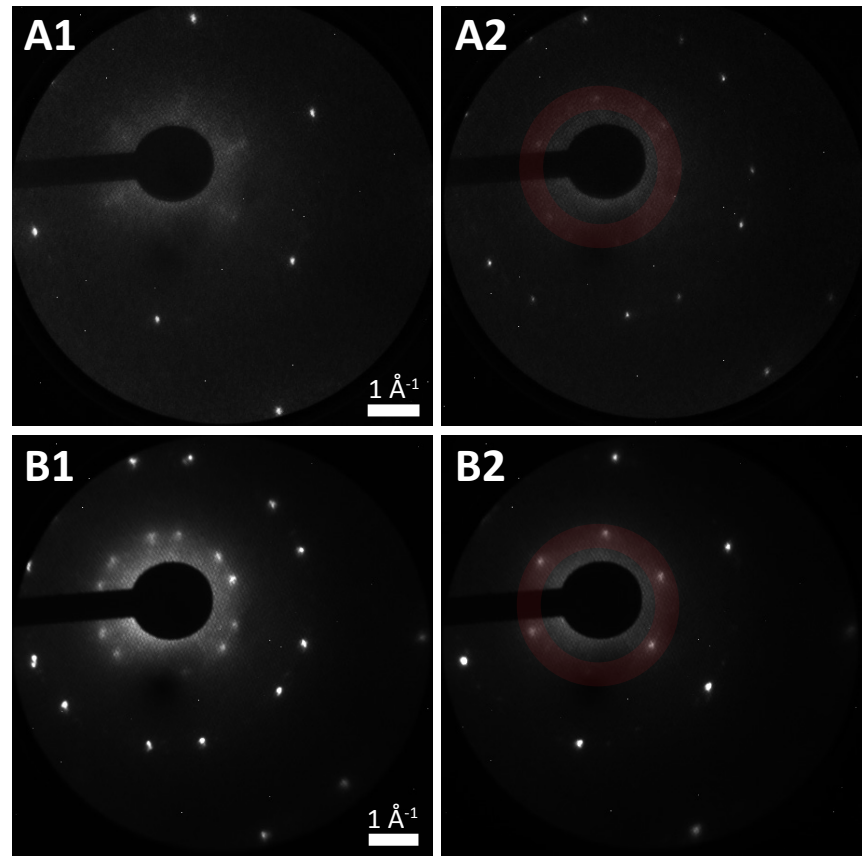

FiguRE B.3: Influence of drying time on the superstructure visibility (marked by gray ring in images A2 and B2). Numbers $\mathbf{1}$ and $\mathbf{2}$ denote two different sample positions. A: Samples directly analyzed after etching. B: Sample from same batch, but analyzed after one day of drying under atmospheric conditions. Integration times: $3 \mathrm{~s}$ per image for A, 5 s per image for B. Both samples are PMMA.

The inner diffraction peaks close to the beam stop (area indicated by gray ring shape in images Fig. B.3A2) are nearly not discernible in the A samples (low drying time), whereas their intensities are comparable to the graphene spot intensity for the B samples (24 h drying time). 


\section{B.3 Thermal resistance of the superstructure}

The polymer superstructure is prepared on many different samples and found to be extremely resistant to thermal and chemical treatment with various solvents [105, 111]. In order to exclude any influence of the Quantifoil film ${ }^{3}$ on the recorded structures, several samples are prepared on $\mathrm{Si}_{x} \mathrm{~N}_{y}$ membranes ${ }^{4}$ instead. This alteration also allows for bake-out of the samples, which is not possible when using Quantifoil. In the latter case, the carbon / plastic membranes are not thermally stable for temperatures above $150{ }^{\circ} \mathrm{C}$, which results in the destruction of the overlaying graphene layer. Fig. B.4 shows three different graphene / PMMA samples prepared on $\mathrm{Si}_{x} \mathrm{~N}_{y}$ membranes before (1) and after (2) bake-out for $6 \mathrm{~h}$ at $350{ }^{\circ} \mathrm{C}$ to $400{ }^{\circ} \mathrm{C}$ under atmospheric conditions. The polymer superstructure is visible on each sample. Note that the overall signal intensity has degraded significantly after bake-out.
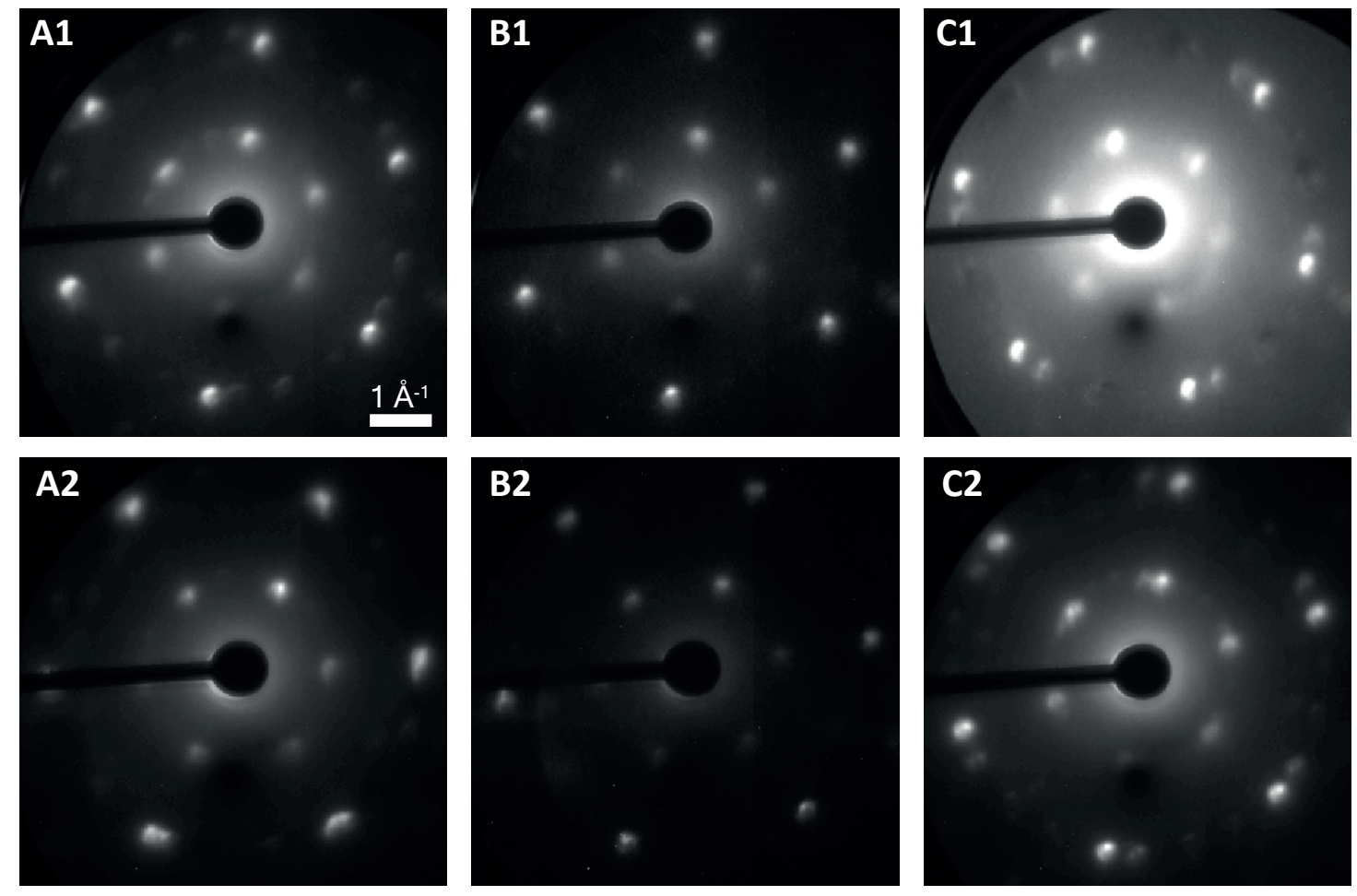

Figure B.4: Sample on SiN before and after bake-out. Top row: PMMA on graphene on SiN-TEM grid (no Quantifoil). Bottom row: Same samples (different sample positions) after bake-out for $6 \mathrm{~h}$ at $350{ }^{\circ} \mathrm{C}$. Overall signal reduction, but superstructure still visible. Integration time: $10 \mathrm{~s}$. Image contrast normalized. Electron energy: $450 \mathrm{eV}$.

\footnotetext{
${ }^{3}$ Quantifoil is produced by evaporation of carbon on a holey plastic film, which is later on dissolved. However, the manufactures claims that not all of the plastic is removed, but about $10 \mathrm{~nm}$ remain below the $10 \mathrm{~nm}$ carbon film (http:// http://www.quantifoil.com/).

${ }^{4}$ Vendor: Plano GmbH (http://www.plano-em.de/)
} 
In the case of PMMA not in contact with a graphene surface, degradation already starts at temperatures as low as $165{ }^{\circ} \mathrm{C}$ by chain scissions at $\mathrm{H}-\mathrm{H}$ linkages [274]. At around $360{ }^{\circ} \mathrm{C}$, random scissions are setting in within the polymer chains. To observe a mere reduction of the overall signal but no removal of the PMMA at such high temperatures indicated the strong influence of the graphene surface potential on the PMMA stability.

\section{B.4 Superstructure degradation}

There are two main contributions to the degradation of the superstructure diffraction signal, namely electron bombardment by the gun and continuous heating by the intense laser pump pulse. The first contribution can be estimated by considering the lifetime of the superstructure in a TEM and comparing the experimental conditions to the ULEED setup. As mentioned, the polymer superstructure lasts only for about $1-2 \mathrm{~s}$ in a TEM and even the low-dose, low-temperature approach of a cryo-TEM cannot increase the lifetime to beyond $10 \mathrm{~s}$.

The main reason for this short lifetime is the small area in which the electrons are focused within in a TEM. While the electron current is distributed over an area with about $60-70 \mu \mathrm{m}$ in diameter in the case of ULEED (Sec. 5.2), TEMs have focal diameters in the order of $5 \mathrm{~nm}$ and below, leading to maximum current densities of about $10^{6} \mathrm{~A} / \mathrm{m}^{2}$. This figure is more than 10 orders of magnitude higher than that for ULEED. In terms of electrons per unit cell per second, this estimate means that whereas in ULEED, each PMMA unit cell is hit by an electron within the timespan of about one hour, electrons bombard the unit cell every few seconds in a TEM even at reduced current densities and taking into account the reduced scattering cross-section at higher energies [275]. This can lead to a series of physical and chemical processes eventually degrading the sample signal [225].

The second important source of superstructure degradation stems from the heating of the sample by the intense pump laser beam. The superstructure lattice parameter as well as diffraction spot width and intensity as a function of total integration time for low fluences are displayed in Fig. B.5A. While the change in the measured lattice parameter is about $1 \%$ (red squares), the relative spot intensity (blue circles) and peak width (green triangles) change significantly. After $60 \mathrm{~min}$, only $70 \%$ of the initial diffraction 
intensity of the spots is left, while the peak has experienced a broadening by about $5-10 \%$.
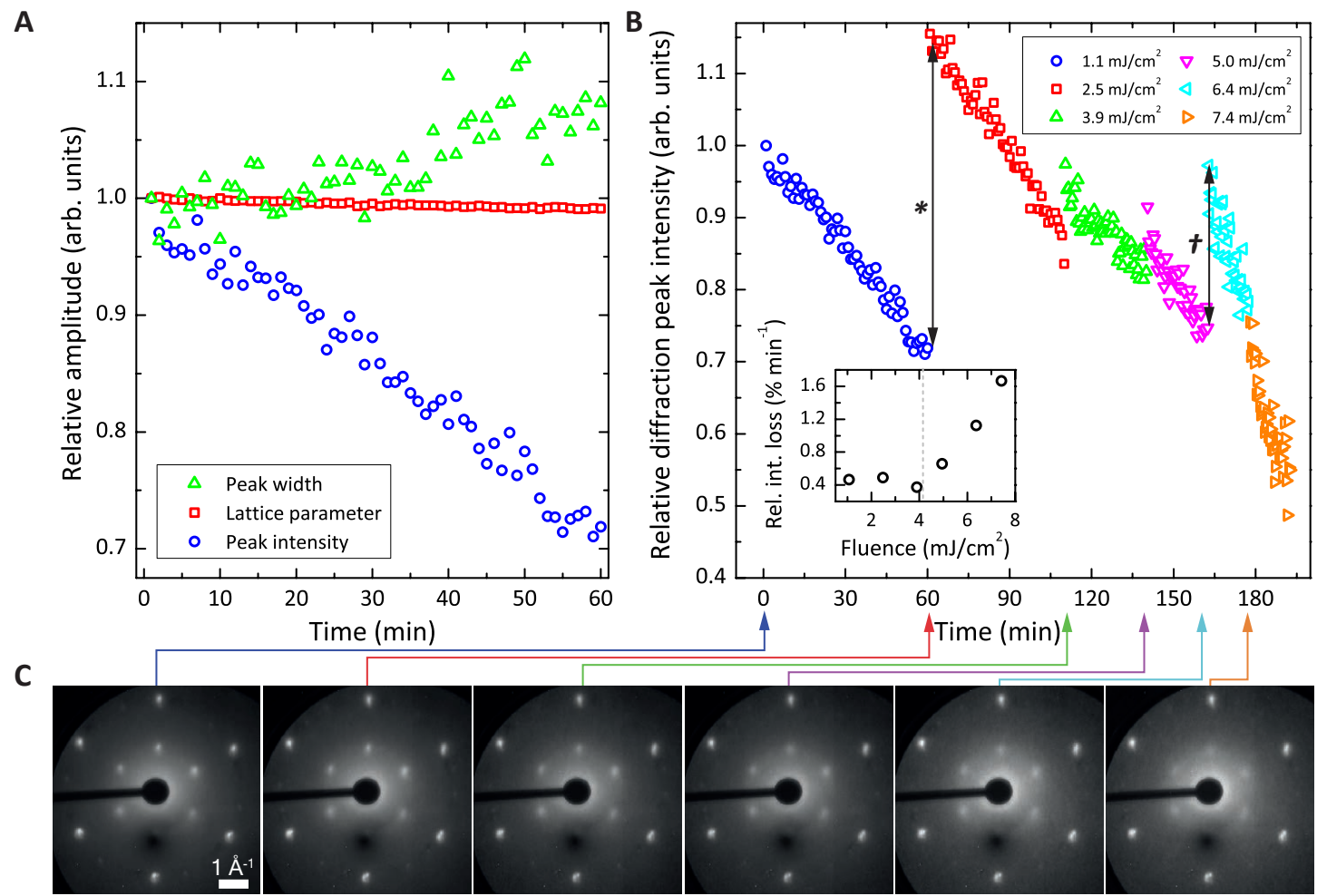

Figure B.5: Superstructure degradation. Electron energy: $450 \mathrm{eV}$. Pump laser repetition rate: $312 \mathrm{kHz}$. All measurement at negative delay times. A: Low-fluence development of the superstructure peak width, peak position and the lattice parameter. Pump fluence: $1.1 \mathrm{~mJ} / \mathrm{cm}^{2}$. B: Intensity development as a function of laser pump fluence. The asterisk $\left(^{*}\right)$ and dagger $(\dagger)$ indicate slight sample movement to increase overall intensity. Inset: Amplitude decrease as a function of pump fluence. Threshold fluence (estimated from visible onset of lost crystalline order) for superstructure melting indicated by gray dashed line. C: Diffraction images taken at the start of each measurement.

When increasing the pump fluence, the rate of intensity loss first remains about constant up to $4 \mathrm{~mJ} / \mathrm{cm}^{2}$ and strongly increases for even higher fluences (Fig. B.5B) up to about $2 \%$ per minute. The intensity loss as a function of fluence is displayed in the inset of Fig. B.5B. The respective diffraction images are shown in Fig. B.5C, recorded at large positive delay times to exclude any effects from the relaxation process of the superstructure (Sec. 5.2).

Notably, the intensity loss is significantly enhanced at fluences beyond a threshold ${ }^{5}$ of $4 \mathrm{~mJ} / \mathrm{cm}^{2}$, with the onset of the loss of crystalline order in the superstructure. In

\footnotetext{
${ }^{5}$ The higher observed threshold fluence (compare Sec. 6.3.4) is most likely caused by slight variations between different sample sites or a small defocussing of the pump beam on the sample.
} 
addition to mere sample degradation, also non-complete recrystallization of the superstructure amounts to the higher observed intensity loss. Also, a dependence of the degradation velocity on the electron energy has not been observed. However, a possible larger inelastic energy transfer on the sample could be masked by a reduced scattering cross section of faster electrons [275]. 



\section{Appendix C}

\section{Abbreviations and symbols}

\section{C.1 Abbreviations}

$\begin{array}{ll}\text { AES } & \text { Auger electron spectroscopy } \\ \text { AFM } & \text { atomic force microscopy } \\ \text { BBO } & \text { B-barium borate } \\ \text { BSCCO } & \text { bismuth strontium calcium copper oxide } \\ \text { CCD } & \text { charge-coupled device } \\ \text { CNT } & \text { carbon nanotube } \\ \text { CVD } & \text { chemical vapor deposition } \\ \text { DI } & \text { de-ionized } \\ \text { FEM } & \text { finite element method } \\ \text { FWHM } & \text { full width at half maximum } \\ \text { HOPG } & \text { highly ordered pyrolytic graphite } \\ \text { IMFP } & \text { inelastic mean-free-path } \\ \text { I-V } & \text { intensity-energy / intensity-velocity } \\ \text { LEED } & \text { low-energy electron diffraction } \\ \text { MCP } & \text { microchannel plate } \\ \text { MMA } & \text { methyl-methacrylate } \\ \text { OPA } & \text { optical parametric amplifier } \\ \text { PC } & \text { polycarbonate } \\ \text { PE } & \text { polyethylene } \\ \text { PINEM } & \text { photon-induced near field electron microscopy } \\ & \end{array}$


PMMA poly(methyl methacrylate)

RHEED reflection high-energy electron diffraction

RMS root-mean-square

sCDW surface charge density wave

SEM scanning electron microscopy

SHG second-harmonic generation

STM scanning tunneling microscopy

TEF transient electric field

TEM transmission electron microscope

THz-STM terahertz STM

trARPES time and angle-resolved photoemission spectroscopy

UED ultrafast electron diffraction

ULEED ultrafast LEED

UTEM ultrafast TEM

XRD X-ray diffraction

\section{C.2 Symbols}

$\begin{array}{ll}c & \text { speed of light }(299792458 \mathrm{~m} / \mathrm{s}) \\ e & \text { charge of an electron }\left(1.60217657 \cdot 10^{-19} \mathrm{C}\right) \\ \epsilon_{0} & \text { vacuum permittivity }\left(8.85418782 \cdot 10^{-12} \mathrm{~F} / \mathrm{m}\right) \\ \hbar & \text { Planck's constant }\left(6.62606957 \cdot 10^{-34} \mathrm{~m}^{2} \mathrm{~kg} / \mathrm{s}\right) \\ k_{B} & \text { Boltzmann constant }\left(1.3806488 \cdot 10^{-23} \mathrm{~m}^{2} \mathrm{~kg} / \mathrm{s}^{2} \mathrm{~K}\right) \\ m_{e} & \text { mass of an electron }\left(9.10938291 \cdot 10^{-31} \mathrm{~kg}\right) \\ \mathbb{1} & \quad \text { identity matrix } \\ A & \text { area } \\ a & \text { lattice parameter } \\ \mathbf{a}_{i} & \text { lattice vector } \\ \mathbf{a}_{i}^{*} & \text { reciprocal lattice parameter } \\ B & \text { brightness } \\ \mathbf{b}_{i} & \text { overlayer lattice parameter } \\ \mathbf{b}_{i}^{*} & \text { reciprocal overlayer lattice vector }\end{array}$


$c_{G} \quad$ specific heat capacity

d distance, thickness

$D \quad$ distribution

E energy

$F \quad$ electric field

$F_{h k} \quad$ structure factor

$f_{h k} \quad$ structure factor coefficient

$\mathbf{g}_{h k} \quad$ reciprocal lattice vector

$h, k, l \quad$ Miller indices

$I \quad$ intensity

$K \quad$ force

$\mathbf{k}, \mathbf{s} \quad$ wave vectors

$L \quad$ diameter of light source

$L_{m} \quad$ specific latent heat for melting

$l_{c} \quad$ transverse coherence length

M mass

$N_{e} \quad$ electron number

n surface normal vector

$R \quad$ distance between light source and scattering object

$R_{k} \quad$ thermal boundary resistance

$r \quad$ radius, distance

$\dot{r}, v \quad$ velocity

$T$ temperature

$t \quad$ time

$U$ potential

$U_{p} \quad$ ponderomotive potential

$\alpha \quad$ angle, expansion coefficient

$\beta \quad$ angle, relativistic factor

$\Delta E \quad$ energy spread

$\Delta t \quad$ delay time

$\delta_{i j} \quad$ Dirac delta function

$\epsilon \quad$ emittance 


$\begin{array}{ll}\epsilon_{F} & \text { energy of Fermi level } \\ \epsilon_{V} & \text { energy of vacuum level } \\ \gamma & \text { Keldysh parameter, photon energy, relativistic factor } \\ \lambda & \text { wave length } \\ \Phi & \text { work function } \\ \varphi & \text { angle, phase coefficient } \\ \Omega & \text { solid angle } \\ \omega & \text { angular frequency } \\ \rho & \text { charge / mass density } \\ \sigma & \text { standard deviation } \\ \Theta & \text { diffraction angle, Heaviside function } \\ \tau & \text { time duration }\end{array}$




\section{Bibliography}

[1] H. Lüth. Solid surfaces, interfaces and thin films. Springer, 4th edition, 2001.

[2] K. Oura, V. G. Lifshits, A. Saranin, A. V. Zotov, and M. Katayama. Surface Science: An introdcution. Springer Berlin Heidelberg, 1st edition, 2003.

[3] S. Y. Tong. Electron-diffraction for surface studies - the first 30 years. Surface Science, 299-300(93):358-374, 1994. URL http://www. sciencedirect.com/ science/article/pii/003960289490667X.

[4] G. Binnig, C. F. Quate, and C. Gerber. Atomic force microscope. Physical Review Letters, 56(9):930-933, 1986. URL http://prl.aps.org/abstract/PRL/v56/i9/ p930_1.

[5] C. Davisson and L. H. Germer. Diffraction of electrons by a crystal of nickel. Physical Review, 30(6):705-740, 1927. URL http://prola.aps.org/abstract/ $\mathrm{PR} / \mathrm{v} 30 / \mathrm{i} 6 / \mathrm{p} 705$ _1.

[6] L. J. Clarke. Surface crystallography: an introduction to low energy electron diffraction. John Wiley \& Sons Ltd, 1st edition, 1985.

[7] D. C. Joy. Scanning electron microscopy. Wiley-VCH Verlag GmbH \& Co. KGaA, 1st edition, 2006.

[8] J. Tersoff and D. R. Hamann. Theory of the scanning tunneling microscope. Physical Review B, 31(2):805-813, 1985. URL http://journals.aps.org/prb/ abstract/10.1103/PhysRevB.31.805.

[9] W. Friedrich, P. Knipping, and M. Laue. Interferenz-Erscheinungen bei Röentgenstrahlen. Sitzungsberichte der Mathematisch-Physikalischen Classe der Königlich-Bayerischen Akademie der Wissenschaften zu Muenchen, pages 303322,1912 . 
[10] N. Camillone, C. E. D. Chidsey, G. Liu, and G. Scoles. Superlattice structure at the surface of a monolayer of octadecanethiol self-assembled on $\mathrm{Au}(111)$. The Journal of Chemical Physics, 98(4):3503-3511, 1993. ISSN 00219606. URL http: //link. aip.org/link/JCPSA6/v98/i4/p3503/s1\&Agg=doi.

[11] L. Fu and C. Kane. Probing Neutral Majorana Fermion Edge Modes with Charge Transport. Physical Review Letters, 102(21):216403, May 2009. ISSN 0031-9007. URL http://link.aps.org/doi/10.1103/PhysRevLett.102.216403.

[12] A. Y. Kitaev. Unpaired Majorana fermions in quantum wires. Physics-Uspekhi, 44:131-136, 2001. URL http://iopscience.iop.org/1063-7869/44/10S/S29.

[13] V. Mourik, K. Zuo, S. M. Frolov, S. R. Plissard, E. P. A. M. Bakkers, and L. P. Kouwenhoven. Signatures of Majorana fermions in hybrid superconductorsemiconductor nanowire devices. Science, 336(6084):1003-7, May 2012. ISSN 1095-9203. URL http://www.ncbi.nlm.nih.gov/pubmed/22499805.

[14] G. A. Somorjai and Y. Li. Introduction to Surface Chemistry and Catalysis. John Wiley \& Sons Ltd, 1st edition, 2010.

[15] G. Che, B. B. Lakshmi, E. R. Fisher, and C. R. Martin. Carbon nanotubule membranes for electrochemical energy storage and production. Nature, 393:346349, 1998. URL http://www.nature.com/nature/journal/v393/n6683/abs/ 393346a0.html.

[16] N. M. Markovic and T. J. Schmidt. Oxygen reduction reaction on Pt and Pt bimetallic surfaces: A selective review. Fuel Cells, 1(2):105-116, 2001. URL http://www.researchgate.net/publication/228553301_Oxygen_reduction_ reaction_on_Pt_and_Pt_bimetallic_surfaces_a_selective_review/file/ $72 \mathrm{e} 7 \mathrm{e} 5290 \mathrm{e} 5 \mathrm{~b} 2853 \mathrm{c} 4$.pdf.

[17] R. C. Sousa and I. L. Prejbeanu. Non-volatile magnetic random access memories (MRAM). Comptes Rendus Physique, 6(9):1013-1021, 2005. URL http://www . sciencedirect.com/science/article/pii/S1631070505001519.

[18] A. K. Geim and I. V. Grigorieva. Van der Waals heterostructures. Nature, 499 (7459):419-25, July 2013. ISSN 1476-4687. URL http://www.ncbi.nlm.nih. gov/pubmed/23887427. 
[19] K. S. Novoselov, V. I. Fal'ko, L. Colombo, P. R. Gellert, M. G. Schwab, and K. Kim. A roadmap for graphene. Nature, 490(7419):192-200, October 2012. ISSN 1476-4687. URL http://www.ncbi.nlm.nih.gov/pubmed/23060189.

[20] A. K. Geim. Random walk to graphene. International Journal of Modern Physics B, 25(30):4055-4080, 2011. URL http://www.worldscientific.com/doi/pdf/ 10.1142/S0217979211059097.

[21] T. Ramanathan, A. A. Abdala, S. Stankovich, D. A. Dikin, M. Herrera-Alonso, R. D. Piner, D. H. Adamson, H. C. Schniepp, X. Chen, R. S. Ruoff, S. T. Nguyen, I. A. Aksay, R. K. Prud'Homme, and L. C. Brinson. Functionalized graphene sheets for polymer nanocomposites. Nature Nanotechnology, 3(6):327-31, June 2008. ISSN 1748-3395. URL http://www.ncbi.nlm.nih.gov/pubmed/18654541.

[22] J. M. Kosterlitz and D. J. Thouless. Ordering, metastability and phase transitions in two-dimensional systems. Journal of Physics C: Solid State Physics, 6(7):11811203, April 1973. ISSN 0022-3719. URL http://stacks.iop.org/0022-3719/6/ $i=7 / a=010$ ?key=crossref.$f 2 d 443370878 b 9288 c 142 e 398 a d 429 b 1$.

[23] Z. Hadzibabic, P. Krüger, M. Cheneau, B. Battelier, and J. Dalibard. BerezinskiiKosterlitz-Thouless crossover in a trapped atomic gas. Nature, 441(7097):111821, June 2006. ISSN 1476-4687. URL http://www.ncbi.nlm.nih.gov/pubmed/ 16810249.

[24] A. M. Kadin, K. Epstein, and A. M. Goldman. Renormalization and the KosterlitzThouless transition in a two-dimensional superconductor. Physical Review B, 27(11):6691-6702, 1983. URL http://prb.aps.org/abstract/PRB/v27/i11/ p6691_1.

[25] D. D. Chambliss. Nucleation of Ordered Ni Island Arrays on Au(111) by SurfaceLattice Dislocations. Physical Review Letters, 66(13):1721-1724, 1991. URL http: //journals.aps.org/prl/abstract/10.1103/PhysRevLett.66.1721.

[26] M. Bienfait. Surface premelting of CH4 thin films. EPL (Europhysics Letters), 4 (1):79-84, 1987. URL http://iopscience.iop.org/0295-5075/4/1/013.

[27] D. B. Pengra, D. M. Zhu, and J. G. Dash. Surface melting of strained and unstrained layers: Kr, Ar, and Ne. Surface Science, 245(1-2):125-131, April 
1991. ISSN 00396028. URL http://linkinghub.elsevier.com/retrieve/pii/ 0039602891904736.

[28] N. Marzari, D. Vanderbilt, A. De Vita, and M. Payne. Thermal Contraction and Disordering of the $\mathrm{Al}(110)$ Surface. Physical Review Letters, 82(16):32963299, April 1999. ISSN 0031-9007. URL http://link.aps.org/doi/10.1103/ PhysRevLett.82.3296.

[29] J. M. Carpinelli, H. H. Weitering, E. W. Plummer, and R. Stumpf. Direct observation of a surface charge density wave. Nature, 381:398-400, 1996. URL http:// www . phys.lsu .edu/plummer/downloads/paper-surfacechargedenswave.pdf.

[30] F. Carbone, O.-H. Kwon, and A. H. Zewail. Dynamics of chemical bonding mapped by energy-resolved 4D electron microscopy. Science, 325(5937):181-4, July 2009. ISSN 1095-9203. URL http://www.ncbi.nlm.nih.gov/pubmed/19589997.

[31] M. Cho. Coherent two-dimensional optical spectroscopy. Chemical Reviews, 108 (4):1331-418, April 2008. ISSN 0009-2665. URL http://pubs .acs .org/doi/abs/ 10.1021/cr078377b? journalCode=chreay.

[32] M. Chergui. Picosecond and femtosecond X-ray absorption spectroscopy of molecular systems. Acta Crystallographica. Section A, Foundations of Crystallography, 66(Pt 2):229-39, March 2010. ISSN 1600-5724. URL http://www.ncbi.nlm.nih. gov/pubmed/20164646.

[33] B. J. Siwick, J. R. Dwyer, R. E. Jordan, and R. J. D. Miller. An atomic-level view of melting using femtosecond electron diffraction. Science, 302(5649):1382-5, November 2003. ISSN 1095-9203. URL http://www.ncbi.nlm.nih.gov/pubmed/ 14631036.

[34] M. Eichberger, M. Krumova, M. Beyer, J. Demsar, H. Berger, G. Moriena, G. Sciaini, and R. J. D. Miller. Snapshots of cooperative atomic motions in the optical suppression of charge density waves. Nature, 468(7325):799-802, December 2010. ISSN 1476-4687. URL http://www.ncbi.nlm.nih.gov/pubmed/21107321.

[35] P. Baum, D.-S. Yang, and A. H. Zewail. 4D visualization of transitional structures in phase transformations by electron diffraction. Science, 318(5851):788-92, November 2007. ISSN 1095-9203. URL http://www.ncbi.nlm.nih.gov/pubmed/ 17975063. 
[36] A. H. Zewail. Four-dimensional electron microscopy. Science, 328(5975):18793, April 2010. ISSN 1095-9203. URL http://www.ncbi.nlm.nih.gov/pubmed/ 20378810.

[37] T. LaGrange, G. H. Campbell, B. W. Reed, M. Taheri, J. B. Pesavento, J. S. Kim, and N. D. Browning. Nanosecond time-resolved investigations using the in situ of dynamic transmission electron microscope (DTEM). Ultramicroscopy, 108(11): 1441-9, October 2008. ISSN 0304-3991. URL http://www.ncbi.nlm.nih.gov/ pubmed/18783886.

[38] O. F. Mohammed, Di.-S. Yang, S. K. Pal, and A. H. Zewail. 4D scanning ultrafast electron microscopy: visualization of materials surface dynamics. Journal of the American Chemical Society, 133(20):7708-11, May 2011. ISSN 1520-5126. URL http://www.ncbi.nlm.nih.gov/pubmed/21539369.

[39] K. B. Eisenthal. Liquid Interfaces Probed by Second-Harmonic and SumFrequency Spectroscopy. Chemical Reviews, 96(4):1343-1360, June 1996. ISSN 1520-6890. URL http://www.ncbi.nlm.nih.gov/pubmed/11848793.

[40] S. Johnson, E. Vorobeva, P. Beaud, C. Milne, and G. Ingold. Full Reconstruction of a Crystal Unit Cell Structure during Coherent Femtosecond Motion. Physical Review Letters, 103(20):205501, November 2009. ISSN 0031-9007. URL http: //link.aps.org/doi/10.1103/PhysRevLett.103.205501.

[41] D. M. Fritz, D. A. Reis, B. Adams, R. A. Akre, J. Arthur, C Blome, P. H. Bucksbaum, A. L. Cavalieri, S. Engemann, S. Fahy, R. W. Falcone, P. H. Fuoss, K. J. Gaffney, M. J. George, J. Hajdu, M. P. Hertlein, P. B. Hillyard, M. Horn-von Hoegen, M. Kammler, J. Kaspar, R. Kienberger, P. Krejcik, S. H. Lee, A. M. Lindenberg, B. McFarland, D. Meyer, T. Montagne, E. D. Murray, A. J. Nelson, M. Nicoul, R. Pahl, J. Rudati, H. Schlarb, D. P. Siddons, K. Sokolowski-Tinten, T. Tschentscher, D. von der Linde, and J B Hastings. Ultrafast bond softening in bismuth: mapping a solid's interatomic potential with X-rays. Science, 315(5812):633-6, February 2007. ISSN 1095-9203. URL http://www.ncbi.nlm.nih.gov/pubmed/17272718.

[42] U. Hofer. Time-Resolved Coherent Photoelectron Spectroscopy of Quantized Electronic States on Metal Surfaces. Science, 277(5331):1480-1482, September 1997. 
ISSN 00368075. URL http://www.sciencemag.org/cgi/doi/10.1126/science. 277.5331 .1480 .

[43] W. E. King, G. H. Campbell, A. Frank, B. Reed, J. F. Schmerge, B. J. Siwick, B. C. Stuart, and P. M. Weber. Ultrafast electron microscopy in materials science, biology, and chemistry. Journal of Applied Physics, 97(11):111101, 2005. ISSN 00218979. URL http://link.aip.org/link/JAPIAU/v97/i11/p111101/ s1\&Agg=doi.

[44] A. Hanisch-Blicharski, A. Janzen, B. Krenzer, S. Wall, F. Klasing, A. Kalus, T. Frigge, M. Kammler, and M. Horn-von Hoegen. Ultra-fast electron diffraction at surfaces: from nanoscale heat transport to driven phase transitions. Ultramicroscopy, 127:2-8, April 2013. ISSN 1879-2723. URL http://www.ncbi.nlm.nih. gov/pubmed/22975358.

[45] P. Zhou, C. Streubühr, A. Kalus, T. Frigge, S. Wall, A. Hanisch-Blicharski, M. Kammler, M. Ligges, U. Bovensiepen, D. von der Linde, and M. Hornvon Hoegen. Ultrafast time resolved reflection high energy electron diffraction with tilted pump pulse fronts. EPJ Web of Conferences, 41:10016, March 2013. ISSN 2100-014X. URL http://www.epj-conferences.org/10.1051/epjconf/ 20134110016.

[46] B. J. Siwick, J. R. Dwyer, R. E. Jordan, and R. J. D. Miller. Ultrafast electron optics: Propagation dynamics of femtosecond electron packets. Journal of Applied Physics, 92(3):1643, 2002. ISSN 00218979. URL http://link.aip.org/link/ JAPIAU/v92/i3/p1643/s1\&Agg=doi.

[47] R. S. Becker, G. S. Higashi, and J. A. Golovchenko. Low-Energy Electron Diffraction during Pulsed Laser Annealing: A Time-Resolved Diffraction Study. Physical Review Letters, 52(4):307-310, 1984. URL http://journals.aps.org/prl/ abstract/10.1103/PhysRevLett.52.307.

[48] M. Krüger, M. Schenk, and P. Hommelhoff. Attosecond control of electrons emitted from a nanoscale metal tip. Nature, 475(7354):78-81, July 2011. ISSN 1476-4687. URL http://www.ncbi.nlm.nih.gov/pubmed/21734706. 
[49] G. Herink, D. R. Solli, M. Gulde, and C. Ropers. Field-driven photoemission from nanostructures quenches the quiver motion. Nature, 483(7388):190193, 2012. ISSN 00280836. URL http://www.nature.com/doifinder/10.1038/ nature10878.

[50] R. Bormann, M. Gulde, A. Weismann, S. Yalunin, and C. Ropers. Tip-Enhanced Strong-Field Photoemission. Physical Review Letters, 105(14):147601, 2010. ISSN 00319007. URL http://link.aps.org/doi/10.1103/PhysRevLett.105.147601.

[51] B. Barwick, C. Corder, J. Strohaber, N. Chandler-Smith, C. Uiterwaal, and H. Batelaan. Laser-induced ultrafast electron emission from a field emission tip. New Journal of Physics, 9(142), May 2007. ISSN 13672630. URL http://stacks.iop.org/1367-2630/9/i=5/a=142?key=crossref . e808ef6132af32ab20f4702ea27e5178.

[52] A. Paarmann, M. Gulde, M. Müller, S. Schäfer, S. Schweda, M. Maiti, C. Xu, T. Hohage, F. Schenk, C. Ropers, and R. Ernstorfer. Coherent femtosecond lowenergy single-electron pulses for time-resolved diffraction and imaging: A numerical study. Journal of Applied Physics, 112(11):113109, 2012. ISSN 00218979. URL http://link.aip.org/link/JAPIAU/v112/i11/p113109/s1\&Agg=doi.

[53] E. Quinonez, J. Handali, and B. Barwick. Femtosecond photoelectron point projection microscope. The Review of Scientific Instruments, 84(10):103710, October 2013. ISSN 1089-7623. URL http://www.ncbi.nlm.nih.gov/pubmed/24182122.

[54] J. C. H. Spence, T. Vecchione, and U. Weierstall. A coherent photofield electron source for fast diffractive and point-projection imaging. Philosophical Magazine, 90(35-36):4691-4702, December 2010. ISSN 1478-6435. URL http: //www.tandf online.com/doi/abs/10.1080/14786431003630868.

[55] L. De Broglie. Recherches sur la théorie des quanta. Phd thesis, Université ParisSorbonne, Paris, 1924. URL http://tel.archives-ouvertes.fr/docs/00/04/ 70/78/PDF/tel-00006807.pdf.

[56] G. P. Thomson and A. Reid. Diffraction of cathode rays by a thin film. Nature, 119(3007):890, 1927. URL http://adsabs.harvard.edu/abs/1927Natur.119Q. $890 \mathrm{~T}$. 
[57] G.P. Thomson. Experiments on the diffraction of cathode rays. Proceedings of the Royal Society A, 117(778):600-609, 1928. URL http://web.pdx.edu/ pmoeck/ pdf/Thomson1928.pdf.

[58] W. L. Bragg. The Specular Reflection of X-rays. Nature, 90(2250):410, 1912. URL http://adsabs.harvard.edu/abs/1912Natur. .90..410B.

[59] P. R. Watson, M. A. Van Hove, and K. Hermann. NIST Surface Structure Database. National Institute of Standards and Technology, 2002. URL http:// chemistry. oregonstate. edu/personalhomepages/Watson/Research/SSD.htm.

[60] P. Auger. Sur les rayons B secondaires produit dans un gaz par des rayons X. Comptes Rendus Physique, 180:65-73, 1925.

[61] M. A. Van Hove, W. H. Weinberg, and C. M. Chan. Low-Energy Electron Diffraction. Springer-Verlag Berlin Heidelberg, 1st edition, 1986.

[62] D. B. Williams, C. B. Carter, and C Barry. Transmission Electron Microscopy: A Textbook for Materials Science. Springer, 2nd edition, 2009.

[63] D. L. Adams, H. B. Nielsen, and M. A. Van Hove. Quantitative analysis of low-energy-electron diffraction: Application to Pt(111). Physical Review B, 20(12):4789-4806, 1979. URL http://journals.aps.org/prb/abstract/10. 1103/PhysRevB.20.4789.

[64] U. Scheithauer, G. Meyer, and M. Henzler. A new LEED instrument for quantitative spot profile analysis. Surface Science, 178(1-3):441-451, 1986. URL http://www.sciencedirect.com/science/article/pii/0039602886903213.

[65] H Minoda, T Shimakura, K Yagi, F Meyer, and M Horn Von Hoegen. Gold-induced faceting on an $\mathrm{Si}(\mathrm{hhm})$ surface $(\mathrm{m} / \mathrm{h}=1.4-1.5)$ studied by spot profile analyzing low-energy electron diffraction. Surface Science, 432(1-2):69-80, 1999. URL http: //www.sciencedirect.com/science/article/pii/S0039602899005166.

[66] M. K. Debe and D. A. King. The reliability of LEED I-V spectra: incidence angle errors and symmetry effects on W $(100)\left(1^{*} 1\right)$. Journal of Physics C: Solid State Physics, 15(10):2257, 1982. URL http://iopscience.iop.org/0022-3719/15/ $10 / 025$. 
[67] G. Held, H. Pfnür, and D. Menzel. A LEED-IV investigation of the Ru (001)p (2x1)-H structure. Surface Science, 271(1-2):21-31, 1992. URL http://www . sciencedirect.com/science/article/pii/0039602892908584.

[68] T. Urano, T. Kanaji, and M. Kaburagi. Surface structure of MgO (001) surface studied by LEED. Surface Science, 134(1):109-121, 1983. URL http://www . sciencedirect.com/science/article/pii/003960288390314X.

[69] M. Henzler and W. Göpel. Oberflächenphysik des Festkörpers. B. G. Teubner, 2nd edition, 1994.

[70] R. Pantel, M. Bujor, and J. Bardolle. Oxygen adsorption on various vicinal faces close to the (0001) basal plane of rhenium. Surface Science, 83(1): 228-242, 1979. URL http://www.sciencedirect.com/science/article/pii/ 0039602879904898.

[71] K. S. Novoselov, A. K. Geim, S. V. Morozov, D. Jiang, Y. Zhang, S. V. Dubonos, I. V. Grigorieva, and A. A. Firsov. Electric field effect in atomically thin carbon films. Science, 306(5696):666-9, October 2004. ISSN 1095-9203. URL http: //www.ncbi.nlm.nih.gov/pubmed/15499015.

[72] A. K. Geim and K. S. Novoselov. The rise of graphene. Nature Materials, 6 (3):183-91, March 2007. ISSN 1476-1122. URL http://www.nature.com/nmat/ journal/v6/n3/abs/nmat1849.html.

[73] A. K. Geim and K. S. Novoselov. Towards Graphene-Based Electronics. Technical report, Centre for Mesoscience \& Nanotechnology, Manchester, 2012. URL http://oai.dtic.mil/oai/oai?verb=getRecord\&metadataPrefix=html\& identifier=ADA554986.

[74] Y. M. Lin, C. Dimitrakopoulos, and K. A. Jenkins. 100-GHz transistors from wafer-scale epitaxial graphene. Science, 327(5966):662, 2010. URL http://www . sciencemag. org/content/327/5966/662. short.

[75] K. Kim, J.-Y. Choi, T. Kim, S.-H. Cho, and H.-J. Chung. A role for graphene in silicon-based semiconductor devices. Nature, 479(7373):338-44, December 2011. ISSN 1476-4687. URL http://www.ncbi.nlm.nih.gov/pubmed/22094694. 
[76] Y.-W. Son, M. L. Cohen, and S. G. Louie. Energy Gaps in Graphene Nanoribbons. Physical Review Letters, 97(21):216803, November 2006. ISSN 0031-9007. URL http://link.aps.org/doi/10.1103/PhysRevLett.97.216803.

[77] H.-Bi. Zhang, Q. Yan, W.-G. Zheng, Z. He, and Z.-Z. Yu. Tough graphenepolymer microcellular foams for electromagnetic interference shielding. ACS Applied Materials \& Interfaces, 3(3):918-24, March 2011. ISSN 1944-8244. URL http://www.ncbi.nlm.nih.gov/pubmed/21366239.

[78] F. Xia, T. Mueller, Y.-M. Lin, Al. Valdes-Garcia, and P. Avouris. Ultrafast graphene photodetector. Nature Nanotechnology, 4(12):839-43, December 2009. ISSN 1748-3395. URL http://www.ncbi.nlm.nih.gov/pubmed/19893532.

[79] S. Stankovich, D. A. Dikin, G. H. B. Dommett, K. M. Kohlhaas, E. J. Zimney, E. A. Stach, R. D. Piner, S. T. Nguyen, and R. S. Ruoff. Graphene-based composite materials. Nature, 442(7100):282-6, July 2006. ISSN 1476-4687. URL http: //www.ncbi.nlm.nih.gov/pubmed/16855586.

[80] Y. Xu, Y. Wang, J. Liang, Y. Huang, Y. Ma, X. Wan, and Y. Chen. A hybrid material of graphene and poly (3,4-ethyldioxythiophene) with high conductivity, flexibility, and transparency. Nano Research, 2(4):343-348, April 2009. ISSN 1998-0124. URL http://link. springer.com/10.1007/s12274-009-9032-9.

[81] Y. R. Lee, A. V. Raghu, H. M. Jeong, and B. K. Kim. Properties of waterborne polyurethane/functionalized graphene sheet nanocomposites prepared by an in situ method. Macromolecular Chemistry and Physics, 2010(15):1247-1254, 2009. URL http://onlinelibrary.wiley.com/doi/10.1002/macp.200900157/full.

[82] H. Quan, B. Zhang, Q. Zhao, R. K. K. Yuen, and R. K. Y. Li. Facile preparation and thermal degradation studies of graphite nanoplatelets (GNPs) filled thermoplastic polyurethane (TPU) nanocomposites. Composites Part A: Applied Science and Manufacturing, 40(9):1506-1513, 2009. URL http://www.sciencedirect. com/science/article/pii/S1359835X09001845.

[83] S. Bae, H. Kim, Y. Lee, X. Xu, J.-S. Park, Yi Zheng, J. Balakrishnan, T. Lei, H. R. Kim, Y. I. Song, Y.-J. Kim, K. S. Kim, B. Ozyilmaz, J.-H. Ahn, B. H. Hong, and S. Iijima. Roll-to-roll production of 30-inch graphene films for transparent 
electrodes. Nature Nanotechnology, 5(8):574-8, August 2010. ISSN 1748-3395. URL http://www.ncbi.nlm.nih.gov/pubmed/20562870.

[84] K. S. Kim, Y. Zhao, H. Jang, S. Y. Lee, J. M. Kim, J.-H. Ahn, P. Kim, J.-Y. Choi, and B. H. Hong. Large-scale pattern growth of graphene films for stretchable transparent electrodes. Nature, 457(7230):706-10, February 2009. ISSN 1476-4687. URL http://www.ncbi.nlm.nih.gov/pubmed/19145232.

[85] J. Ryu, Y. Kim, D. Won, N. Kim, J. S. Park, E.-K. Lee, D. Cho, S.-P. Cho, S. J. Kim, G. H. Ryu, H.-A-S. Shin, Z. Lee, B. H. Hong, and S. Cho. Fast synthesis of high-performance graphene films by hydrogen-free rapid thermal chemical vapor deposition. ACS Nano, 8(1):950-6, January 2014. ISSN 1936-086X. URL http: //www.ncbi.nlm.nih.gov/pubmed/24358985.

[86] A. Bostwick, T. Ohta, T. Seyller, K. Horn, and E. Rotenberg. Quasiparticle dynamics in graphene. Nature Physics, 3(1):36-40, December 2007. ISSN 17452473. URL http://www. nature.com/doifinder/10.1038/nphys477.

[87] T. Trauzettel. Von Graphit zu Graphen. Physik Journal, 6(7):39-44, 2007. URL www.pro-physik.de/details/articlePdf/1104875/issue.html.

[88] Y. Baskin and L. Meyer. Lattice constants of graphite at low temperatures. Physical Review, 100(2):544, 1955. URL http://prola.aps.org/abstract/PR/v100/ i2/p544_1.

[89] O. V. Yazyev and S. G. Louie. Topological defects in graphene: Dislocations and grain boundaries. Physical Review B, 81(19):195420, May 2010. ISSN 1098-0121. URL http://link.aps.org/doi/10.1103/PhysRevB.81.195420.

[90] P. Y. Huang, C. S. Ruiz-Vargas, A. M. van der Zande, W. S. Whitney, M. P. Levendorf, J. W. Kevek, S. Garg, J. S. Alden, C. J. Hustedt, Y. Zhu, J. Park, P. L. McEuen, and D. A. Muller. Grains and grain boundaries in single-layer graphene atomic patchwork quilts. Nature, 469(7330):389-92, January 2011. ISSN 1476-4687. URL http://www.ncbi.nlm.nih.gov/pubmed/21209615.

[91] R. Grantab, V. B. Shenoy, and R. S. Ruoff. Anomalous strength characteristics of tilt grain boundaries in graphene. Science, 330(6006):946-8, November 2010. ISSN 1095-9203. URL http://www.ncbi.nlm.nih.gov/pubmed/21071664. 
[92] L. A. Ponomarenko, A. K. Geim, A. A. Zhukov, R. Jalil, S. V. Morozov, K. S. Novoselov, I. V. Grigorieva, E. H. Hill, V. V. Cheianov, V. I. Fal'ko, K. Watanabe, T. Taniguchi, and R. V. Gorbachev. Tunable metal-insulator transition in double-layer graphene heterostructures. Nature Physics, 7(12):958-961, October 2011. ISSN 1745-2473. URL http://www.nature.com/doifinder/10.1038/ nphys 2114.

[93] S. J. Haigh, A. Gholinia, R. Jalil, S. Romani, L. Britnell, D. C. Elias, K. S. Novoselov, L. A. Ponomarenko, A. K. Geim, and R. Gorbachev. Cross-sectional imaging of individual layers and buried interfaces of graphene-based heterostructures and superlattices. Nature Materials, 11(9):764-7, September 2012. ISSN 1476-1122. URL http://www.ncbi.nlm.nih.gov/pubmed/22842512.

[94] L. Britnell, R. V. Gorbachev, R. Jalil, B. D. Belle, F. Schedin, A. Mishchenko, T. Georgiou, M. I. Katsnelson, L. Eaves, S. V. Morozov, N. M. R. Peres, J. Leist, A. K. Geim, K. S. Novoselov, and L. A. Ponomarenko. Field-effect tunneling transistor based on vertical graphene heterostructures. Science, 335(6071):947-50, February 2012. ISSN 1095-9203. URL http://www.ncbi.nlm.nih.gov/pubmed/ 22300848.

[95] T. K. Das and S. Prusty. Graphene-Based Polymer Composites and Their Applications. Polymer-Plastics Technology and Engineering, 52(4):319-331, March 2013. ISSN 0360-2559. URL http://www.tandfonline.com/doi/abs/10.1080/ 03602559.2012 .751410 .

[96] T. Hasan, V. Scardaci, P. H. Tan, F. Bonaccorso, A. G. Rozhin, Z. Sun, and A. C. Ferrari. Molecular- and Nano-Tubes. Springer, 1st edition, 2011.

[97] K. S. Choi, F. Liu, J. S. Choi, and T. S. Seo. Fabrication of free-standing multilayered graphene and poly(3,4-ethylenedioxythiophene) composite films with enhanced conductive and mechanical properties. Langmuir, 26(15):12902-8, August 2010. ISSN 1520-5827. URL http://www.ncbi.nlm.nih.gov/pubmed/20617852.

[98] J. Du and H. M. Cheng. The fabrication, properties, and uses of graphene/polymer composites. Macromolecular Chemistry and Physics, 213(10-111):1060-1077, 2012. URL http://onlinelibrary.wiley.com/doi/10.1002/macp. 201200029/full. 
[99] P. V. Kamat. Graphene-Based Nanoassemblies for Energy Conversion. The Journal of Physical Chemistry Letters, 2(3):242-251, February 2011. ISSN 1948-7185. URL http://pubs.acs.org/doi/abs/10.1021/jz101639v.

[100] Y. Sun and G. Shi. Graphene/polymer composites for energy applications. Journal of Polymer Science Part B: Polymer Physics, 51(4):231-253, February 2013. ISSN 08876266. URL http://doi.wiley.com/10.1002/polb. 23226.

[101] S. Lara-Avila, K. Moth-Poulsen, R. Yakimova, T. Bjø rnholm, V. Fal'ko, A. Tzalenchuk, and S. Kubatkin. Non-volatile photochemical gating of an epitaxial graphene/polymer heterostructure. Advanced Materials, 23(7):878-82, February 2011. ISSN 1521-4095. URL http://www.ncbi.nlm.nih.gov/pubmed/21328484.

[102] X. Li, Y. Zhu, W. Cai, M. Borysiak, B. Han, D. Chen, R. D. Piner, L. Colombo, and R. S. Ruoff. Transfer of large-area graphene films for high-performance transparent conductive electrodes. Nano Letters, 9(12):4359-63, December 2009. ISSN 1530-6992. URL http://www.ncbi.nlm.nih.gov/pubmed/19845330.

[103] A. Reina, X. Jia, J. Ho, D. Nezich, H. Son, V. Bulovic, M. S. Dresselhaus, and J. Kong. Large area, few-layer graphene films on arbitrary substrates by chemical vapor deposition. Nano Letters, 9(1):30-5, January 2009. ISSN 1530-6984. URL http://www.ncbi.nlm.nih.gov/pubmed/19046078.

[104] Y.-C. Lin, C. Jin, J.-C. Lee, S.-F. Jen, K. Suenaga, and P.-W. Chiu. Clean transfer of graphene for isolation and suspension. ACS Nano, 5(3):2362-8, March 2011. ISSN 1936-086X. URL http://www.ncbi.nlm.nih.gov/pubmed/21351739.

[105] Y. C. Lin, C. C. Lu, C. H. Yeh, and C. Jin. Graphene annealing: how clean can it be? Nano Letters, 12(1):414-419, 2012. URL http://pubs.acs.org/doi/abs/ $10.1021 / \mathrm{n} 1203733 \mathrm{r}$.

[106] H. J. Park, J. Meyer, S. Roth, and V. Skákalová. Growth and properties of few-layer graphene prepared by chemical vapor deposition. Carbon, 48 (4):1088-1094, 2010. URL http://www.sciencedirect.com/science/article/ $\mathrm{pii/S0008622309007659.}$

[107] W. Regan, N. Alem, B. Alemán, B. Geng, C. Girit, L. Maserati, F. Wang, M. Crommie, and A. Zettl. A direct transfer of layer-area graphene. Applied 
Physics Letters, 96(11):113102, 2010. ISSN 00036951. URL http://scitation. aip.org/content/aip/journal/apl/96/11/10.1063/1.3337091.

[108] T. Kuilla, S. Bhadra, D. Yao, and N. H. Kim. Recent advances in graphene based polymer composites. Progress in Polymer Science, 35(11):1350-1375, 2010. URL http://www.sciencedirect.com/science/article/pii/S0079670010000699.

[109] A. N. Rissanou and V. Harmandaris. Dynamics of various polymer-graphene interfacial systems through atomistic molecular dynamics simulations. Soft Matter, 10(16):2876-88, April 2014. ISSN 1744-6848. URL http://www.ncbi.nlm.nih. gov/pubmed/24667937.

[110] J. S. Ha, H.-S. Roh, S.-D. Jung, S.-J. Park, J.-J. Kim, and E.-H. Lee. Structural study of a poly(methylmethacrylate) Langmuir-Blodgett film on a graphite surface by scanning tunneling microscope. Journal of Vacuum Science 83 Technology B: Microelectronics and Nanometer Structures, 12(3):1977, May 1994. ISSN 0734211X. URL http://link. aip.org/link/?JVB/12/1977/1\&Agg=doi.

[111] Y. Takanashi and J. Kumaki. Significant Melting Point Depression of TwoDimensional Folded- Chain Crystals of Isotactic Poly(methyl methacrylate)s Observed by High-Resolution In Situ Atomic Force Microscopy. Physical Chemistry B, 117(18):5594-5605, 2013. URL http://www.ncbi.nlm.nih.gov/pubmed/ 23614490 .

[112] G. C. McGonigal, R. H. Bernhardt, Y. H. Yeo, and D. J. Thomson. Observation of highly ordered, two-dimensional n-alkane and n-alkanol structures on graphite. Journal of Vacuum Science \& Technology B, 9(2):1107, March 1991. ISSN 0734211X. URL http://scitation.aip.org/content/avs/journal/jvstb/9/ 2/10.1116/1.585270.

[113] D. Hoffman and L. Miller. Kinetics of crystallization from the melt and chain folding in polyethylene fractions revisited: theory and experiment. Polymer, 38(13): 3151-3212, 1997. URL http://www.sciencedirect.com/science/article/ pii/S0032386197000712.

[114] H. Ibach. Physics of Surfaces and Interfaces. Springer, 1st edition, 2006. 
[115] R. H. G. Brinkhuis and A. J. Schouten. Thin-film behavior of poly (methyl methacrylates). 1. Monolayers at the air-water interface. Macromolecules, 24(7): 1487-1495, 1991. URL http://pubs.acs.org/doi/abs/10.1021/ma00007a009.

[116] R. H. G. Brinkhuis and A. J. Schouten. Thin-film behavior of poly (methyl methacrylates). 2. An FT-IR study of Langmuir-Blodgett films of isotactic PMMA. Macromolecules, 24(7):1496-1504, 1991. URL http://pubs.acs.org/doi/abs/ 10.1021/ma00007a010.

[117] A. J. DeMaria, W. H. Glenn, M. J. Brienza, and M. E. Mack. Picosecond laser pulses. Proceedings of the IEEE, 57(1):2-25, 1969. ISSN 0018-9219. URL http: //ieeexplore. ieee.org/lpdocs/epic03/wrapper.htm?arnumber=1448794.

[118] P. M. Rentzepis. Ultrafast processes. Science, 169(3942):239-247, 1970. URL http://www. sciencemag. org/content/169/3942/239. short.

[119] G. R. Fleming, J. M. Morris, and G. W. Robinson. Direct observation of rotational diffusion by picosecond spectroscopy. Chemical Physics, 17(1):91-100, 1976. URL http://www.sciencedirect.com/science/article/pii/0301010476850094.

[120] A. A. Ischenko, V. V. Golubkov, V. P. Spiridonov, A. V. Zgurskii, A. S. Akhmanov, M. G. Vabischevich, and V. N. Bagratashvili. A stroboscopical gas-electron diffraction method for the investigation of short-lived molecular species. Applied Physics B, 32(3):161-163, November 1983. ISSN 0721-7269. URL http: //link.springer.com/10.1007/BF00688823.

[121] S. Williamson, G. Mourou, and J. C. M. Li. Time-resolved laser-induced phase transformation in aluminum. Physical Review Letters, 52(26):2364-2367, 1984. URL http://prl.aps.org/abstract/PRL/v52/i26/p2364_1.

[122] Y Erkal, EÇ Turfan, E Sert, and G Mermer. The Potential of the Dynamic Transmission Electron Microscope (DTEM) for In Situ Microscopy. Technical report, Faculty of Health Sciences, Konya, 2013. URL http://posteranmeldung. de/tl_files/vortraege/OralPresentations/Oral12Erkal.pdf.

[123] D. Shorokhov and A. H. Zewail. 4D electron imaging: principles and perspectives. Physical Chemistry Chemical Physics, 10(20):2879-93, May 2008. ISSN 1463-9076. URL http://www.ncbi.nlm.nih.gov/pubmed/18473037. 
[124] A. Stolow, A. E. Bragg, and D. M. Neumark. Femtosecond time-resolved photoelectron spectroscopy. Chemical reviews, 104(4):1719-57, April 2004. ISSN 00092665. URL http://www.ncbi.nlm.nih.gov/pubmed/15080710.

[125] F. Schmitt, P. S. Kirchmann, U. Bovensiepen, R. G. Moore, L. Rettig, M. Krenz, J.-H. Chu, N. Ru, L. Perfetti, D. H. Lu, M. Wolf, I. R. Fisher, and Z.-X. Shen. Transient electronic structure and melting of a charge density wave in TbTe3. Science, 321(5896):1649-52, September 2008. ISSN 1095-9203. URL http://www . ncbi.nlm.nih.gov/pubmed/18703710.

[126] L. Perfetti, P. A. Loukakos, M. Lisowski, U. Bovensiepen, H. Eisaki, and M. Wolf. Ultrafast Electron Relaxation in Superconducting Bi2Sr2CaCu2O8 $+\delta$ by TimeResolved Photoelectron Spectroscopy. Physical Review Letters, 99(19):197001, November 2007. ISSN 0031-9007. URL http://link.aps.org/doi/10.1103/ PhysRevLett.99.197001.

[127] J. A. van Bokhoven, C. Louis, J. T. Miller, M. Tromp, O. V. Safonova, and P. Glatzel. Activation of Oxygen on Gold/Alumina Catalysts: In Situ HighEnergy-Resolution Fluorescence and Time-Resolved X-ray Spectroscopy. Angewandte Chemie, 118(28):4767-4770, July 2006. ISSN 0044-8249. URL http: //doi.wiley.com/10.1002/ange.200601184.

[128] K. Sokolowski-Tinten, C. Blome, J. Blums, A. Cavalleri, Cl. Dietrich, Al. Tarasevitch, I. Uschmann, E. Förster, M. Kammler, M. Horn-von Hoegen, and D. von der Linde. Femtosecond X-ray measurement of coherent lattice vibrations near the Lindemann stability limit. Nature, 422(6929):287-9, March 2003. ISSN 0028-0836. URL http://www.ncbi.nlm.nih.gov/pubmed/12646915.

[129] M. Gao, C. Lu, H. Jean-Ruel, L. C. Liu, A. Marx, K. Onda, S.-Y. Koshihara, Y. Nakano, X. Shao, T. Hiramatsu, G. Saito, H. Yamochi, R. R. Cooney, G. Moriena, G. Sciaini, and R. J. D. Miller. Mapping molecular motions leading to charge delocalization with ultrabright electrons. Nature, 496(7445):343-6, April 2013. ISSN 1476-4687. URL http://www.ncbi.nlm.nih.gov/pubmed/23598343.

[130] T. L. Cocker, V. Jelic, M. Gupta, S. J. Molesky, J. A. J. Burgess, G. D. L. Reyes, L. V. Titova, Y. Y. Tsui, M. R. Freeman, and F. A. Hegmann. An ultrafast terahertz scanning tunnelling microscope. Nature Photonics, 7(8):620-625, July 2013. 
ISSN 1749-4885. URL http://www.nature.com/doifinder/10.1038/nphoton. 2013.151.

[131] B. W. Reed, M. R. Armstrong, N. D. Browning, G. H. Campbell, J. E. Evans, T. LaGrange, and D. J. Masiel. The evolution of ultrafast electron microscope instrumentation. Microscopy and Microanalysis, 15(4):272-81, August 2009. ISSN 1435-8115. URL http://www.ncbi.nlm.nih.gov/pubmed/19575828.

[132] S. Schäfer, W. Liang, and A. H. Zewail. Structural dynamics of nanoscale gold by ultrafast electron crystallography. Chemical Physics Letters, 515(4-6):278282, October 2011. ISSN 00092614. URL http://linkinghub.elsevier.com/ retrieve/pii/S0009261411011699.

[133] M. Nicoul, U. Shymanovich, A. Tarasevitch, D. von der Linde, and K. SokolowskiTinten. Picosecond acoustic response of a laser-heated gold-film studied with time-resolved x-ray diffraction. Applied Physics Letters, 98(19):191902, 2011. ISSN 00036951. URL http://scitation.aip.org/content/aip/journal/apl/ 98/19/10.1063/1.3584864.

[134] B. Krenzer, A. Hanisch-Blicharski, P. Schneider, T. Payer, S. Möllenbeck, O. Osmani, M. Kammler, R. Meyer, and M. Horn-von Hoegen. Phonon confinement effects in ultrathin epitaxial bismuth films on silicon studied by time-resolved electron diffraction. Physical Review B, 80(2):024307, July 2009. ISSN 1098-0121. URL http://link.aps.org/doi/10.1103/PhysRevB.80.024307.

[135] W. Liang, S. Schäfer, and A. H. Zewail. Ultrafast electron crystallography of monolayer adsorbates on clean surfaces: Structural dynamics. Chemical Physics Letters, 542:1-7, July 2012. ISSN 00092614. URL http://www.sciencedirect. com/science/article/pii/S0009261412006501.

[136] U. Dahmen, S. Hagege, and F. Faudot. Observations of interface premelting at grain-boundary precipitates of $\mathrm{Pb}$ in Al. Philosophical Magazine, 84(25-26), 2004. URL http://www.tandfonline.com/doi/abs/10.1080/ 14786430410001671403.

[137] R. Ernstorfer, M. Harb, C. T. Hebeisen, G. Sciaini, T. Dartigalongue, and R. J. D. Miller. The formation of warm dense matter: experimental evidence for electronic 
bond hardening in gold. Science, 323(5917):1033-7, February 2009. ISSN 10959203. URL http://www.ncbi.nlm.nih.gov/pubmed/19164708.

[138] M. Gao, H. Jean-Ruel, and R. R. Cooney. Full characterization of RF compressed femtosecond electron pulses using ponderomotive scattering. Optics Express, 20(11):799-802, 2012. URL http://www.opticsinfobase.org/abstract. cfm?uri=oe-20-11-12048.

[139] G. Sciaini and R. J. D. Miller. Femtosecond electron diffraction: heralding the era of atomically resolved dynamics. Reports on Progress in Physics, 74(9):096101, September 2011. ISSN 0034-4885. URL http://stacks.iop.org/0034-4885/ $74 / i=9 / a=096101$ ?key=crossref $.5 c f 8$ eb1cb398a8048108bdb5d3ce79e3.

[140] P. Baum. On the physics of ultrashort single-electron pulses for timeresolved microscopy and diffraction. Chemical Physics, 423:55-61, September 2013. ISSN 03010104. URL http://linkinghub.elsevier.com/retrieve/pii/ S0301010413002681.

[141] C. Cirelli, M. Hengsberger, A. Dolocan, H. Over, J. Osterwalder, and T. Greber. Direct observation of space charge dynamics by picosecond low-energy electron scattering. EPL (Europhysics Letters), 85(1):17010, January 2009. ISSN 0295-5075. URL http://stacks.iop.org/0295-5075/85/i=1/a=17010? key=crossref . Oada5732e7a0a20a3c25f58fd3693781.

[142] R. Karrer, H. J. Neff, M. Hengsberger, T. Greber, and J. Osterwalder. Design of a miniature picosecond low-energy electron gun for time-resolved scattering experiments. Review of Scientific Instruments, 72(12):4404, 2001. ISSN 00346748. URL http://link.aip.org/link/RSINAK/v72/i12/p4404/s1\&Agg=doi.

[143] A. Janzen, B. Krenzer, O. Heinz, P. Zhou, D. Thien, A. Hanisch, F.-J. Meyer Zu Heringdorf, D. von der Linde, and M. Horn von Hoegen. A pulsed electron gun for ultrafast electron diffraction at surfaces. The Review of Scientific Instruments, 78(1):013906, January 2007. ISSN 0034-6748. URL http://www.ncbi.nlm.nih. gov/pubmed/17503932.

[144] H. Nörenberg, R. Säverin, U. Hoppe, and G. Holzhüter. Estimation of radial distribution functions in electron diffraction experiments: physical, mathematical 
and numerical aspects. Journal of Applied Crystallography, 32(5):911-916, October 1999. ISSN 00218898. URL http://scripts.iucr.org/cgi-bin/paper? S0021889899006603.

[145] T. LaGrange, M. R. Armstrong, K. Boyden, C. G. Brown, G. H. Campbell, J. D. Colvin, W. J. DeHope, A. M. Frank, D. J. Gibson, F. V. Hartemann, J. S. Kim, W. E. King, B. J. Pyke, B. W. Reed, M. D. Shirk, R. M. Shuttlesworth, B. C. Stuart, B. R. Torralva, and N. D. Browning. Single-shot dynamic transmission electron microscopy. Applied Physics Letters, 89(4):044105, 2006. ISSN 00036951. URL http://link. aip.org/link/APPLAB/v89/i4/p044105/s1\&Agg=doi.

[146] G Dehm, JM Howe, and J Zweck. In-situ Electron Microscopy: Applications in Physics, Chemistry and Materials Science. Wiley-VCH Verlag GmbH \& Co. KGaA, 1st edition, 2012.

[147] P. Musumeci, J. T. Moody, C. M. Scoby, M. S. Gutierrez, H. A. Bender, and N. S. Wilcox. High quality single shot diffraction patterns using ultrashort megaelectron volt electron beams from a radio frequency photoinjector. The Review of Scientific Instruments, 81(1):013306, January 2010. ISSN 1089-7623. URL http://www . ncbi.nlm.nih.gov/pubmed/20113092.

[148] G. F. Mancini, B. Mansart, S. Pagano, B. van der Geer, M. de Loos, and F. Carbone. Design and implementation of a flexible beamline for fs electron diffraction experiments. Nuclear Instruments and Methods in Physics Research Section A, 691:113-122, November 2012. ISSN 01689002. URL http: //linkinghub.elsevier.com/retrieve/pii/S0168900212007334.

[149] P. Musumeci, J. T. Moody, C. M. Scoby, M. S. Gutierrez, and M. Westfall. Laserinduced melting of a single crystal gold sample by time-resolved ultrafast relativistic electron diffraction. Applied Physics Letters, 97(6):063502, 2010. ISSN 00036951. URL http://link.aip.org/link/APPLAB/v97/i6/p063502/s1\&Agg= doi.

[150] J. Yang, N. Naruse, and K. Kan. Femtosecond electron guns for ultrafast electron diffraction. IPAC Proceedings, pages 4170-4174, 2012. URL https://accelconf . web.cern.ch/accelconf/IPAC2012/papers/frxbb01.pdf. 
[151] J. B. Hastings, F. M. Rudakov, D. H. Dowell, J. F. Schmerge, J. D. Cardoza, J. M. Castro, S. M. Gierman, H. Loos, and P. M. Weber. Ultrafast time-resolved electron diffraction with megavolt electron beams. Applied Physics Letters, 89(18): 184109, 2006. ISSN 00036951. URL http://link.aip.org/link/APPLAB/v89/ $\mathrm{i} 18 / \mathrm{p} 184109 / \mathrm{s} 1 \&$ Agg=doi.

[152] J. C. Williamson, M. Dantus, S. B. Kim, and A. H. Zewail. Ultrafast diffraction and molecular structure. Chemical Physics Letters, 196(6):529-534, 1992. URL http://www. sciencedirect.com/science/article/pii/000926149285988M.

[153] Ahmed Zewail. $4 D$ Electron Microscopy: Imaging in Space and Time. World Scientific Publishing Co. Pte. Ltd., 1st edition, 2010.

[154] M. E. Haine and P. A. Einstein. Characteristics of the hot cathode electron microscope gun. British Journal of Applied Physics, 3(2):40-46, 1952. URL http://iopscience.iop.org/0508-3443/3/2/302.

[155] E. L. Murphy and R. H. Good Jr. Thermionic emission, field emission, and the transition region. Physical Review, 102(6):1464-1473, 1956. URL http://prola. aps.org/abstract/PR/v102/i6/p1464_1.

[156] D. H. Dowell and J. F. Schmerge. Quantum efficiency and thermal emittance of metal photocathodes. Physical Review Special Topics - Accelerators and Beams, 12(7):074201, July 2009. ISSN 1098-4402. URL http://link.aps.org/doi/10 . 1103/PhysRevSTAB.12.074201.

[157] A. Bouhelier, M. Beversluis, A. Hartschuh, and L. Novotny. Near-Field SecondHarmonic Generation Induced by Local Field Enhancement. Physical Review Letters, 90(1):013903, January 2003. ISSN 0031-9007. URL http://link.aps.org/ doi/10.1103/PhysRevLett.90.013903.

[158] L. Novotny, R. X. Bian, and X. S. Xie. Theory of nanometric optical tweezers. Physical Review Letters, 79(4):645-648, July 1997. ISSN 0031-9007. URL http: //link.aps.org/doi/10.1103/PhysRevLett.79.645.

[159] P. F. Liao and A. Wokaun. Lightning rod effect in surface enhanced Raman scattering. The Journal of Chemical Physics, 76(1):751, 1982. ISSN 00219606. URL http: //scitation.aip.org/content/aip/journal/jcp/76/1/10.1063/1.442690. 
[160] R. Gomer. Field emission and field ionization. Havard University Press, 1st edition, 1961.

[161] H. D. Beckey, H. Krone, and F. W. Roellgen. Comparison of tips, thin wires and sharp metal edges as emitters for field ionization mass spectrometry. Journal of Physics E, 118, 1968. URL http://iopscience.iop.org/0022-3735/1/2/308.

[162] J. Orloff. Handbook of charged particle optics. CRC Press, 2nd edition, 2008.

[163] J. A. Berger, J. T. Hogan, M. J. Greco, W. A. Schroeder, A. W. Nicholls, and N. D. Browning. DC photoelectron gun parameters for ultrafast electron microscopy. Microscopy and Microanalysis, 15(4):298-313, August 2009. ISSN 1435-8115. URL http://www.ncbi.nlm.nih.gov/pubmed/19575831.

[164] M. Aeschlimann and E. Hull. A picosecond electron gun for surface analysis. Review of Scientific Instruments, 66:1000-1009, 1995. URL http://scitation. aip.org/content/aip/journal/rsi/66/2/10.1063/1.1146036.

[165] A. Gahlmann, S. Tae Park, and A. H. Zewail. Ultrashort electron pulses for diffraction, crystallography and microscopy: theoretical and experimental resolutions. Physical Chemistry Chemical Physics, 10(20):2894-909, May 2008. ISSN 1463-9076. URL http://www.ncbi.nlm.nih.gov/pubmed/18473038.

[166] M. Merano, S. Collin, P. Renucci, M. Gatri, S. Sonderegger, A. Crottini, Ganière J. D., and B. Deveaud. High brightness picosecond electron gun. Review of Scientific Instruments, 76(8):085108, 2005. ISSN 00346748. URL http://scitation.aip. org/content/aip/journal/rsi/76/8/10.1063/1.2008975.

[167] Note. Images D-F courtesy of Georg Herink (IV. Physical Institute, University of Göttingen), adapted from [49].

[168] P. Hommelhoff, C. Kealhofer, A. Aghajani-Talesh, Y. R. P. Sortais, S. M. Foreman, and M. A. Kasevich. Extreme localization of electrons in space and time. Ultramicroscopy, 109(5):423-9, April 2009. ISSN 0304-3991. URL http: //www.ncbi.nlm.nih.gov/pubmed/19117677.

[169] N. Behr and M. B. Raschke. Optical Antenna Properties of Scanning Probe Tips: Plasmonic Light Scattering, Tip-Sample Coupling, and Near-Field Enhancement. 
Journal of Physical Chemistry C, 112(10):3766-3773, March 2008. ISSN 19327447. URL http://pubs.acs.org/doi/abs/10.1021/jp7098009.

[170] S. Irvine, A. Dechant, and A. Elezzabi. Generation of 0.4-keV Femtosecond Electron Pulses using Impulsively Excited Surface Plasmons. Physical Review Letters, 93(18):184801, October 2004. ISSN 0031-9007. URL http://link.aps.org/doi/ 10.1103/PhysRevLett.93.184801.

[171] P. Hommelhoff, Y. Sortais, A. Aghajani-Talesh, and M. A. Kasevich. Field Emission Tip as a Nanometer Source of Free Electron Femtosecond Pulses. Physical Review Letters, 96(7):077401, February 2006. ISSN 0031-9007. URL http://link.aps.org/doi/10.1103/PhysRevLett.96.077401.

[172] C Ropers. Femtosecond excitations in metallic nanostructures. Phd thesis, Humboldt-Universität zu Berlin, Berlin, 2007. URL http://edoc.hu-berlin. de/dissertationen/ropers-claus-2007-07-11/METADATA/abstract.php?id= 28147.

[173] H. R. Reiss. Effect of an intense electromagnetic field on a weakly bound system. Physical Review A, 22(5):1786-1813, 1980. URL http://pra.aps.org/abstract/ PRA/v22/i5/p1786_1.

[174] S. V. Yalunin, G. Herink, D. R. Solli, M. Krüger, P. Hommelhoff, M. Diehn, A. Munk, and C. Ropers. Field localization and rescattering in tip-enhanced photoemission. Annalen der Physik, 525(1-2):L12-L18, February 2013. ISSN 00033804. URL http://doi.wiley.com/10.1002/andp. 201200224.

[175] S. V. Yalunin, M. Gulde, and C. Ropers. Strong-field photoemission from surfaces: Theoretical approaches. Physical Review B, 84(19):1-14, 2011. ISSN 10980121. URL http://link.aps.org/doi/10.1103/PhysRevB.84.195426.

[176] Note. Images adapted from Simon Schweda (IV. Physical Institute, University of Göttingen) [191].

[177] W. Lauterborn and T. Kurz. Coherent optics: fundamentals and applications. Springer, 2nd edition, 2003.

[178] A. M. Michalik, E. Y. Sherman, and J. E. Sipe. Theory of ultrafast electron diffraction: The role of the electron bunch properties. Journal of Applied Physics, 104 
(5):054905, 2008. ISSN 00218979. URL http://scitation.aip.org/content/ aip/journal/jap/104/5/10.1063/1.2973157.

[179] Note. Images A and B adapted from [177] and [191], respectively.

[180] W. Demtröder. Experimentalphysik 2 - Elektrizität und Optik. Springer, 6th edition, 2013.

[181] M. Reiser. Theory and design of charged particle beams. Wiley-VCH Verlag GmbH \& Co. KGaA, 1sr edition, 2008.

[182] Stefanie Strauch. Tip-based photoelectron sources for ultrafast transmission electron microscopy. Master thesis, Universität Göttingen, Göttingen, 2013.

[183] M. C. Gordillo and J. Marti. Water on graphene surfaces. Journal of Physics: Condensed Matter, 22(28):284111, July 2010. ISSN 1361-648X. URL http:// www.ncbi.nlm.nih.gov/pubmed/21399283.

[184] O.-H. Kwon and B. Barwick. 4D visualization of embryonic, structural crystallization by single-pulse microscopy. Proceedings of the National Academy of Sciences of the United States of America, 105(25):8519-8524, 2008. URL http://www . pnas . org/content/105/25/8519. short.

[185] R. Bormann. Lokalisierte Femtosekunden-Photoemission aus nanometrischen Goldspitzen. Diploma thesis, Universität Göttingen, Göttingen, 2010.

[186] Max Gulde. Optisch induzierte Feldemission an metallischen Nanostrukturen. Diploma thesis, Universität Göttingen, Göttingen, 2010.

[187] H. Yanagisawa, M. Hengsberger, D. Leuenberger, M. Klöckner, C. Hafner, T. Greber, and J. Osterwalder. Energy Distribution Curves of Ultrafast Laser-Induced Field Emission and Their Implications for Electron Dynamics. Physical Review Letters, 107(8):087601, August 2011. ISSN 0031-9007. URL http://link.aps. org/doi/10.1103/PhysRevLett.107.087601.

[188] M. Schenk, M. Kr, M. Krüger, and P. Hommelhoff. Strong-Field Above-Threshold Photoemission from Sharp Metal Tips. Physical Review Letters, 105(25):257601, December 2010. ISSN 0031-9007. URL http://link.aps.org/doi/10.1103/ PhysRevLett. 105.257601. 
[189] P. J. Bryant, H. S. Kim, Y. C. Zheng, and R. Yang. Technique for shaping scanning tunneling microscope tips. Review of Scientific Instruments, 58(6):1115, 1987. ISSN 00346748. URL http://scitation.aip.org/content/aip/journal/ rsi/58/6/10.1063/1.1139618.

[190] Note. Image courtesy of Simon Schweda (IV. Physical Institute, University of Göttingen).

[191] Simon Schweda. Entwicklung eines Experiments zur zeitaufgelösten Beugung niederenergetischer Elektronen. Master thesis, Universität Göttingen, Göttingen, 2013.

[192] K. C. Mishra, R. Garner, and P. C. Schmidt. Model of work function of tungsten cathodes with barium oxide coating. Journal of Applied Physics, 95(6):3069, 2004. ISSN 00218979. URL http://scitation.aip.org/content/aip/journal/jap/ 95/6/10.1063/1.1646451.

[193] S.-H. Kim, H. Otsuka, H.-W. Shin, K. Tanaka, R. C. Advincula, and H. Usui. Manipulation of Work Function and Surface Free Energy of Tungsten Oxide Hole Injection Layer Modified with a Self-Assembled Monolayer. Japanese Journal of Applied Physics, 50(1S2):01BB01, January 2011. ISSN 0021-4922. URL http: //stacks.iop.org/1347-4065/50/01BB01.

[194] J. G. Fujimoto and E. P. Ippen. Femtosecond laser interaction with metallic tungsten and nonequilibrium electron and lattice temperatures. Physical Review Letters, 53(19):1837-1840, 1984. URL http://adsabs.harvard.edu/abs/ 1984PhRvL. . 53.1837F.

[195] B. Rethfeld, A. Kaiser, M. Vicanek, and G. Simon. Femtosecond laser-induced heating of electron gas in aluminium. Applied Physics A, 69(1):109-112, 1999. URL http://link.springer.com/article/10.1007/s003399900228.

[196] R. Yen, J. Liu, and N. Bloembergen. Thermally assisted multiphoton photoelectric emission from tungsten. Optics Communications, 35(2):277-282, 1980. URL http: //www.sciencedirect.com/science/article/pii/003040188090228X. 
[197] V. T. Binh, N. Garcia, and S. T. Purcell. Electron Field Emission from Atom-Sources: Fabrication, Properties, and Applications of Nanotips. Advances in Imaging and Electron Physics, 95:63-153, 1996. URL http://www. sciencedirect.com/science/article/pii/S1076567008701563.

[198] C. Ropers, T. Elsaesser, G. Cerullo, M. Zavelani-Rossi, and C. Lienau. Ultrafast optical excitations of metallic nanostructures: from light confinement to a novel electron source. New Journal of Physics, 9(10):397-397, October 2007. ISSN 13672630. URL http://stacks.iop.org/1367-2630/9/i=10/a=397?key=crossref . $495913 c 28004$ e470fd663e4af84509d4.

[199] J. R. Dwyer, C. T. Hebeisen, R. Ernstorfer, M. Harb, V. B. Deyirmenjian, R. E. Jordan, and R. J. D. Miller. Femtosecond electron diffraction: 'making the molecular movie'. Philosophical Transactions. Series A, Mathematical, Physical, and Engineering Sciences, 364(1840):741-78, March 2006. ISSN 1364-503X. URL http://www.ncbi.nlm.nih.gov/pubmed/16483961.

[200] H. Park, Z. Hao, X. Wang, S. Nie, R. Clinite, and J. Cao. Synchronization of femtosecond laser and electron pulses with subpicosecond precision. Review of Scientific Instruments, 76(8):083905, 2005. ISSN 00346748. URL http://link. aip.org/link/RSINAK/v76/i8/p083905/s1\&Agg=doi.

[201] T. L. Gilton, J. P. Cowin, G. D. Kubiak, and A. V. Hamza. Intense surface photoemission: Space charge effects and self-acceleration. Journal of Applied Physics, 68 (9):4802, 1990. ISSN 00218979. URL http://link.aip.org/link/JAPIAU/v68/ i9/p4802/s1\&Agg=doi.

[202] H. Park and J.-M. Zuo. Direct measurement of transient electric fields induced by ultrafast pulsed laser irradiation of silicon. Applied Physics Letters, 94(25): 251103, 2009. ISSN 00036951. URL http://link.aip.org/link/APPLAB/v94/ i25/p251103/s1\&Agg=doi.

[203] S. Schäfer, W. Liang, and A. H. Zewail. Primary structural dynamics in graphite. New Journal of Physics, 13(6):063030, June 2011. ISSN 1367-2630. URL http://stacks.iop.org/1367-2630/13/i=6/a=063030?key= crossref.1bb24b4813109185ed905889068d87de. 
[204] H. Park and J.-M. Zuo. Comment on "Structural Preablation Dynamics of Graphite Observed by Ultrafast Electron Crystallography". Physical Review Letters, 105(5):059603, July 2010. ISSN 0031-9007. URL http://link.aps.org/ doi/10.1103/PhysRevLett.105.059603.

[205] J. Cao, Z. Hao, H. Park, C. Tao, D. Kau, and L. Blaszczyk. Femtosecond electron diffraction for direct measurement of ultrafast atomic motions. Applied Physics Letters, 83(5):1044, 2003. ISSN 00036951. URL http://link.aip.org/link/ APPLAB/v83/i5/p1044/s1\&Agg=doi.

[206] M. Eichberger, N. Erasmus, K. Haupt, G. Kassier, A. von Flotow, J. Demsar, and H. Schwoerer. Femtosecond streaking of electron diffraction patterns to study structural dynamics in crystalline matter. Applied Physics Letters, 102(12): 121106, 2013. ISSN 00036951. URL http://link.aip.org/link/APPLAB/v102/ $i 12 / \mathrm{p} 121106 / \mathrm{s} 1 \&$ Agg $=$ doi.

[207] R. Li, W. Huang, Y. Du, L. Yan, Q. Du, J. Shi, J. Hua, H. Chen, T. Du, H. Xu, and C. Tang. Note: Single-shot continuously time-resolved MeV ultrafast electron diffraction. The Review of Scientific Instruments, 81(3):036110, March 2010. ISSN 1089-7623. URL http://www.ncbi.nlm.nih.gov/pubmed/20370233.

[208] F. O. Kirchner, A. Gliserin, F. Krausz, and P. Baum. Laser streaking of free electrons at $25 \mathrm{keV}$. Nature Photonics, 8(1):52-57, December 2013. ISSN 17494885. URL http://www.nature.com/doifinder/10.1038/nphoton.2013.315.

[209] C. T. Hebeisen, R. Ernstorfer, M. Harb, T. Dartigalongue, R. E. Jordan, and R. J. D. Miller. Femtosecond electron pulse characterization using laser ponderomotive scattering. Optics letters, 31(23):3517-9, December 2006. ISSN 0146-9592. URL http://www.ncbi.nlm.nih.gov/pubmed/17099769.

[210] B. J. Siwick, A. A. Green, C. T. Hebeisen, and R. J. D. Miller. Characterization of ultrashort electron pulses by electron-laser pulse cross correlation. Optics Letters, 30(9):1057-9, May 2005. ISSN 0146-9592. URL http://www.ncbi.nlm.nih.gov/ pubmed/15907002.

[211] B. Barwick, D. J. Flannigan, and A. H. Zewail. Photon-induced near-field electron microscopy. Nature, 462(7275):902-6, December 2009. ISSN 1476-4687. doi: 10. 1038/nature08662. URL http://www.ncbi.nlm.nih.gov/pubmed/20016598. 
[212] A. Cavalleri, T. Dekorsy, H. Chong, J. Kieffer, and R. Schoenlein. Evidence for a structurally-driven insulator-to-metal transition in VO2: A view from the ultrafast timescale. Physical Review B, 70(16):161102, October 2004. ISSN 1098-0121. URL http://link.aps.org/doi/10.1103/PhysRevB.70.161102.

[213] M. Breusing, C. Ropers, and T. Elsaesser. Ultrafast carrier dynamics in graphite. Physical Review Letters, 102(8):086809, February 2009. ISSN 0031-9007. URL http://link.aps.org/doi/10.1103/PhysRevLett.102.086809.

[214] M. Drescher, M. Hentschel, R. Kienberger, M. Uiberacker, V. Yakovlev, A. Scrinzi, T. Westerwalbesloh, U. Kleineberg, U. Heinzmann, and F. Krausz. Time-resolved atomic inner-shell spectroscopy. Nature, 419(6909):803-7, October 2002. ISSN 0028-0836. URL http://www.ncbi.nlm.nih.gov/pubmed/12397349.

[215] M. Müller, A. Paarmann, and R. Ernstorfer. Femtosecond electrons probing currents and atomic structure in nanomaterials. arXiv preprint, pages 1-15, 2014. URL http://arxiv.org/abs/1405.4992.

[216] U. Weigel, D. A. Orlov, and S. N. Kosolobov. Cold intense electron beams from LN2-cooled GaAs-photocathodes. Nuclear Instruments and Methods in Physics Research Section A, 536(11):232-328, 2005. URL http://www.sciencedirect. com/science/article/pii/S0168900204019849.

[217] L. Wimmer, G. Herink, D. R. Solli, S. V. Yalunin, K. E. Echternkamp, and C. Ropers. Terahertz control of nanotip photoemission. Nature Physics, 10:432-436, 2014. URL http://www.nature.com/nphys/journal/v10/n6/full/nphys2974.html.

[218] G. H. Kassier, N. Erasmus, K. Haupt, I. Boshoff, R. Siegmund, S. M. M. Coelho, and H. Schwoerer. Photo-triggered pulsed cavity compressor for bright electron bunches in ultrafast electron diffraction. Applied Physics B, 109(2):249-257, September 2012. ISSN 0946-2171. URL http://link.springer.com/10.1007/ s00340-012-5207-2.

[219] T. van Oudheusden, P. L. E. M. Pasmans, S. B. van der Geer, M. J. de Loos, M. J. van der Wiel, and O. J. Luiten. Compression of Subrelativistic Space-ChargeDominated Electron Bunches for Single-Shot Femtosecond Electron Diffraction. Physical Review Letters, 105(26):264801, December 2010. ISSN 0031-9007. URL http://link.aps.org/doi/10.1103/PhysRevLett.105.264801. 
[220] M. Gulde, S. Schweda, G. Storeck, M. Maiti, H. K. Yu, A. M. Wodtke, S. Schafer, and C. Ropers. Ultrafast low-energy electron diffraction in transmission resolves polymer/graphene superstructure dynamics. Science, 345(6193):200-204, July 2014. ISSN 0036-8075. URL http://www.sciencemag.org/cgi/doi/10.1126/ science. 1250658.

[221] A. N. Rissanou and V. Harmandaris. Structure and dynamics of poly(methyl methacrylate)/graphene systems through atomistic molecular dynamics simulations. Journal of Nanoparticle Research, 15(5):1589, April 2013. ISSN 1388-0764. URL http://link. springer.com/10.1007/s11051-013-1589-2.

[222] H. Wang, J. H. Strait, P. A. George, S. Shivaraman, V. B. Shields, M. Chandrashekhar, J. Hwang, F. Rana, M. G. Spencer, C. S. Ruiz-Vargas, and J. Park. Ultrafast relaxation dynamics of hot optical phonons in graphene. Applied Physics Letters, 96(8):081917, 2010. ISSN 00036951. URL http://scitation.aip.org/ content/aip/journal/apl/96/8/10.1063/1.3291615.

[223] M. H. Gass, U. Bangert, A. L. Bleloch, P. Wang, R. R. Nair, and A. K. Geim. Freestanding graphene at atomic resolution. Nature Nanotechnology, 3(11):676-81, November 2008. ISSN 1748-3395. URL http://www.ncbi.nlm.nih.gov/pubmed/ 18989334.

[224] D. C. Miller, M. D. Kempe, C. E. Kennedy, and S. R. Kurtz. Analysis of Transmitted Optical Spectrum Enabling Accelerated Testing of CPV Designs: Preprint. Society of Photographic Instrumentation Engineers (SPIE), 2009. URL http://www.osti.gov/bridge/product.biblio.jsp?osti_id=962021.

[225] D. T. Grubb. Review Radiation damage and electron microscopy of organic polymers. Journal of Materials Science, 9(10):1715-1736, 1974. URL http: //link.springer.com/article/10.1007/BF00540772.

[226] X. Li, W. Cai, J. An, S. Kim, J. Nah, D. Yang, R. Piner, A. Velamakanni, I. Jung, E. Tutuc, S. K. Banerjee, L. Colombo, and R. S. Ruoff. Large-area synthesis of high-quality and uniform graphene films on copper foils. Science, 324(5932):13124, June 2009. ISSN 1095-9203. URL http://www.ncbi.nlm.nih.gov/pubmed/ 19423775. 
[227] M. J. Allen, V. C. Tung, and R. B. Kaner. Honeycomb carbon: a review of graphene. Chemical Reviews, 110(1):132-45, January 2010. ISSN 1520-6890. URL http://www.ncbi.nlm.nih.gov/pubmed/19610631.

[228] R. Bormann. Development of a tip-based ultrafast TEM setup. Phd thesis (not yet published), University of Göttingen, 2014.

[229] Note. Image A courtesy of Holger Stark (Max Planck Institute for Biophysical Chemistry).

[230] J. Kumaki, T. Kawauchi, and E. Yashima. Two-dimensional folded chain crystals of a synthetic polymer in a Langmuir-Blodgett film. Journal of the American Chemical Society, 127(16):5788-9, April 2005. ISSN 0002-7863. URL http:// www.ncbi.nlm.nih.gov/pubmed/15839667.

[231] J.-J. Kim, S.-D. Jung, H.-S. Roh, and J.-S. Ha. Molecular configuration of isotactic PMMA Langmuir-Blodgett films observed by scanning tunnelling microscopy. Thin Solid Films, 244(1-2):700-704, May 1994. ISSN 00406090. URL http://linkinghub.elsevier.com/retrieve/pii/0040609094905533.

[232] Note. Images in A courtesy of Philip Willke, Philipp Ansorg, Thomas Kotzott, and Martin Wenderoth (IV. Physical Institute, University of Göttingen).

[233] Note. Image C courtesy of Stefanie Strauch, James E. Evans, and Nigel D. Browning. This image is part of the Chemical Imaging Initiative at Pacific Northwest National Laboratory under Contract DE-AC05-76RL01830 operated for DOE by Battelle. A portion of the research was performed using EMSL, a national scientific user facility sponsored by the Department of Energy's Office of Biological and Environmental Research and located at Pacific Northwest National Laboratory.

[234] D. C. Bassett, F. C. Frank, and A. Keller. Some new habit features in crystals of long chain compounds part IV. the fold surface geometry of monolayer polyethylene crystals and its relevance to fold packing and crystal growth. Philosophical Magazine, 8(94):1753-1787, October 1963. ISSN 0031-8086. URL http://www. tandfonline.com/doi/abs/10.1080/14786436308207335.

[235] W. Kleber, H.-J. Bautsch, J. Bohm, and D. Klimm. Einführung in die Kristallographie. Oldenbourg Wissenschaftsverlag, 18th edition, 2002. URL http: //www.tandfonline.com/doi/pdf/10.1080/11035895809447264. 
[236] K. Weishaupt, H. Krbecek, M. Pietralla, H. D. Hochheimer, and P. Mayr. Pressure dependence of the elastic constants of poly (methyl methacrylate). Polymer, 36(17):3267-3271, 1995. URL http://www.sciencedirect.com/science/ article/pii/003238619599424S.

[237] E. Pop, V. Varshney, and A. K. Roy. Thermal properties of graphene: Fundamentals and applications. MRS Bulletin, 37(12):1273-1281, November 2012. ISSN 0883-7694. URL http://www. journals.cambridge.org/abstract_ S0883769412002035.

[238] D. R. Stull and H. Prophet. Natl. Stand. Ref. Data Ser., volume 37. National Institute of Standards and Technology, 2nd edition, 1971. URL www.nist.gov/ data/nsrds/NSRDS-NBS37.pdf.

[239] R. R. Nair, P. Blake, and A. N. Grigorenko. Fine structure constant defines visual transparency of graphene. Science, 320(5881):1308, 2008. URL http://www . sciencemag.org/content/320/5881/1308. short.

[240] J. Brandrup, E. H. Immergut, and E. A. Grulke. Polymer Handbook. John Wiley \& Sons Ltd, 4th edition, 1999.

[241] E. Swartz and R. Pohl. Thermal boundary resistance. Reviews of Modern Physics, 61(3):605-668, July 1989. ISSN 0034-6861. URL http://link.aps.org/doi/10. 1103/RevModPhys.61.605.

[242] D. G. Cahill, W. K. Ford, K. E. Goodson, G. D. Mahan, A. Majumdar, H. J. Maris, R. Merlin, and S. R. Phillpot. Nanoscale thermal transport. Journal of Applied Physics, 93(2):793, 2003. ISSN 00218979. URL http://scitation.aip. org/content/aip/journal/jap/93/2/10.1063/1.1524305.

[243] S. Hida, T. Hori, T. Shiga, J. Elliott, and J. Shiomi. Thermal resistance and phonon scattering at the interface between carbon nanotube and amorphous polyethylene. International Journal of Heat and Mass Transfer, 67:10241029, 2013. ISSN 0017-9310. URL http://www.sciencedirect.com/science/ article/pii/S0017931013007308.

[244] S. T. Huxtable, D. G. Cahill, S. Shenogin, L. Xue, R. Ozisik, P. Barone, M. Usrey, M. S. Strano, G. Siddons, M. Shim, and P. Keblinski. Interfacial heat flow in 
carbon nanotube suspensions. Nature Materials, 2(11):731-4, November 2003. ISSN 1476-1122. URL http://www.ncbi.nlm.nih.gov/pubmed/14556001.

[245] D. Konatham and A. Striolo. Thermal boundary resistance at the graphene-oil interface. Applied Physics Letters, 95(16):163105, 2009. ISSN 00036951. URL http: //scitation.aip.org/content/aip/journal/apl/95/16/10.1063/1.3251794.

[246] J. C. Meyer, A. K. Geim, M. I. Katsnelson, K. S. Novoselov, T. J. Booth, and S. Roth. The structure of suspended graphene sheets. Nature, 446(7131):60-3, March 2007. ISSN 1476-4687. URL http://www.ncbi.nlm.nih.gov/pubmed/ 17330039.

[247] N. D. Mermin. Crystalline order in two dimensions. Physical Review, 176(1): 250-254, 1968. URL http://prola.aps.org/abstract/PR/v176/i1/p250_1.

[248] L. D. Landau. Zur Theorie der Phasenumwandlungen II. Phys. Z. Sowjetunion, 26, 1937.

[249] R. Peierls. Quelques propriétés typiques des corps solides. Annales de l'Institut Henri Poincaré, 3:177-222, 1935. URL https://eudml.org/doc/78996.

[250] V. Tewary and B. Yang. Singular behavior of the Debye-Waller factor of graphene. Physical Review B, 79(12):125416, March 2009. ISSN 1098-0121. URL http: //link.aps.org/doi/10.1103/PhysRevB.79.125416.

[251] B. Shevitski, M. Mecklenburg, W. Hubbard, E. White, B. Dawson, M. Lodge, M. Ishigami, and B. Regan. Dark-field transmission electron microscopy and the Debye-Waller factor of graphene. Physical Review B, 87(4):045417, January 2013. ISSN 1098-0121. URL http://link.aps.org/doi/10.1103/PhysRevB. 87. 045417.

[252] C. H. Lui, K. F. Mak, J. Shan, and T. F. Heinz. Ultrafast Photoluminescence from Graphene. Physical Review Letters, 105(12):127404, September 2010. ISSN 00319007. URL http://link.aps.org/doi/10.1103/PhysRevLett.105.127404.

[253] D. R. Nelson and B. I. Halperin. Dislocation-mediated melting in two dimensions. Physical Review B, 19(5):2457-2484, 1979. URL http://journals .aps.org/prb/ abstract/10.1103/PhysRevB.19.2457. 
[254] E. D. Specht, M. Sutton, R. J. Birgeneau, D. E. Moncton, and P. M. Horn. Phase diagram and phase transitions of krypton on graphite in the one-to-twolayer regime. Physical Review B, 30(3):1589-1592, 1984. URL http://journals. aps.org/prb/abstract/10.1103/PhysRevB.30.1589.

[255] R. Schuster, J. V. Barth, G. Ertl, and R. J. Behm. Scanning Tunneling Microscopy Observation of Phase-Transition Phenomena in the Cs/Cu (110) System: Evidence for a Two-Step Disordering Mechanism of a Uniaxial (1x3) Phase. Physical Review Letters, 69(17):2547-2553, 1992. URL http://journals.aps.org/ prl/abstract/10.1103/PhysRevLett.69.2547.

[256] R. Yen, J. M. Liu, H. Kurz, and N. Bloembergen. Space-time resolved reflectivity measurements of picosecond laser-pulse induced phase transitions in (111) silicon surface layers. Applied Physics A Solids and Surfaces, 27(3):153-160, March 1982. ISSN 0721-7250. URL http://link.springer.com/10.1007/BF00616666.

[257] J. J. Wortman and R. A. Evans. Young's Modulus, Shear Modulus, and Poisson's Ratio in Silicon and Germanium. Journal of Applied Physics, 36(1):153, 1965. ISSN 00218979. URL http://scitation.aip.org/content/aip/journal/jap/ 36/1/10.1063/1.1713863.

[258] G. Andrä, H. D. Geiler, and G. Götz. Explosive Liquid-Phase Crystallization of Thin Silicon Films during Pulse Heating. Physica Status Solidi (a), 74(2): 511-515, 1982. URL http://onlinelibrary.wiley.com/doi/10.1002/pssa. $2210740217 /$ abstract.

[259] A. N. Rissanou and V. Harmandaris. A Molecular Dynamics Study of Polymer/Graphene Nanocomposites. Macromolecular Symposia, 331-332(1):43-49, October 2013. ISSN 10221360. URL http://doi.wiley.com/10.1002/masy. 201300070.

[260] R. R. Cavanagh and D. S. King. Dynamics of nonthermal reactions: Femtosecond surface chemistry. The Journal of Physical Chemistry, 97(4):786-798, 1993. URL http://pubs.acs.org/doi/abs/10.1021/j100106a002.

[261] Note. Images B-D courtesy of Gero Storeck and Simon Schweda (IV. Physical Institute, University of Göttingen).

[262] I. N. Bronštejn, J. Hromkovic, and B. Luderer. Taschenbuch der Mathematik. Verlag Harri Deutsch, 6th edition, 2005. 
[263] T. Fauster, L. Hammer, K. Heinz, and M. A. Schneider. Oberflächenphysik: Grundlagen und Methoden. Oldenbourg Wissenschaftsverlag, 1st edition, 2013.

[264] S. M. Sze, J. L. Moll, and T. Sugano. Range-energy relation of hot electrons in gold. Solid-State Electronics, 7(7):509-523, 1964. URL http://www.sciencedirect. com/science/article/pii/0038110164900887.

[265] M. P. Seah and W. A. Dench. Quantitative electron spectroscopy of surfaces: a standard data base for electron inelastic mean free paths in solids. Surface and Interface Analysis, 1(1):2-11, 1979. URL http://onlinelibrary.wiley.com/ doi/10.1002/sia.740010103/abstract.

[266] J. J. Quinn. Range of excited electrons in metals. Physical Review, 126(4):14531457, May 1962. ISSN 0031-899X. URL http://link.aps.org/doi/10.1103/ PhysRev. 126.1453.

[267] B. A. Szabo and I. Babuška. Finite element analysis. Wiley-Interscience, 1st edition, 1991.

[268] S. Hellmann, M. Beye, C. Sohrt, T. Rohwer, F. Sorgenfrei, H. Redlin, M. Kalläne, M. Marczynski-Bühlow, F. Hennies, M. Bauer, A. Föhlisch, L. Kipp, W. Wurth, and K. Rossnagel. Ultrafast Melting of a Charge-Density Wave in the Mott Insulator 1T-TaS2. Physical Review Letters, 105(18):187401, October 2010. ISSN 00319007. URL http://link.aps.org/doi/10.1103/PhysRevLett.105.187401.

[269] A. C. Ferrari, J. C. Meyer, V. Scardaci, C. Casiraghi, M. Lazzeri, F. Mauri, S. Piscanec, D. Jiang, K. S. Novoselov, S. Roth, and A. K. Geim. Raman Spectrum of Graphene and Graphene Layers. Physical Review Letters, 97(18):1-4, October 2006. ISSN 0031-9007. URL http://link.aps.org/doi/10.1103/PhysRevLett. 97.187401.

[270] AC Ferrari, JC Meyer, and V Scardaci. The Raman Fingerprint of Graphene. arXiv preprint, 2006. URL http://arxiv.org/abs/cond-mat/0606284.

[271] Note. Raman data courtesy of Hakki Yu (Max Planck Institute for Biophyisical Chemistry, Göttingen). 
[272] R. J. Nemanich and S. A. Solin. First-and second-order Raman scattering from finite-size crystals of graphite. Physical Review B, 20(2):392-401, 1979. URL http://prb.aps.org/abstract/PRB/v20/i2/p392_1.

[273] Mi. Xia, Z. Su, and S. Zhang. Raman spectra of bilayer graphene covered with Poly(methyl methacrylate) thin film. AIP Advances, 2(3):032122, 2012. ISSN 21583226. URL http://link.aip.org/link/AAIDBI/v2/i3/p032122/s1\&Agg= doi.

[274] T. Kashiwagi, A. Inaba, J. E. Brown, K. Hatada, T. Kitayama, and E. Masuda. Effects of weak linkages on the thermal and oxidative degradation of poly(methyl methacrylates). Macromolecules, 19(8):2160-2168, August 1986. ISSN 0024-9297. URL http://pubs.acs.org/doi/abs/10.1021/ma00162a010.

[275] K. Kanaya and S. Okayama. Penetration and energy-loss theory of electrons in solid targets. Journal of Physics D: Applied Physics, 5(1):43, 1972. URL http: //iopscience.iop.org/0022-3727/5/1/308. 


\section{Publications and Conferences}

\section{Publications related to this work}

- M. Gulde, S. Schweda, G. Storeck, M. Maiti, H. K. Yu, A. M. Wodtke, S. Schäfer, and C. Ropers, Ultrafast low-energy electron diffraction in transmission resolves polymer/graphene superstructure dynamics, Science 345, 200 (2014).

- A. Paarmann, M. Gulde, M. Müller, S. Schäfer, S. Schweda, M. Maiti, C. Xu, T. Hohage, F. Schenk, C. Ropers, and R. Ernstorfer, Coherent femtosecond low-energy single-electron pulses for time-resolved diffraction and imaging: A numerical study, Journal of Applied Physics 112, 113109 (2012).

- G. Herink, D. R. Solli, M. Gulde, and C. Ropers, Field-driven photoemission from nanostructures quenches the quiver motion, Nature 483, 190 (2012).

- S. V. Yalunin, M. Gulde, and C. Ropers, Strong-field photoemission from surfaces: Theoretical approaches, Phys. Rev. B. 84, 195426 (2011).

- R. Bormann, M. Gulde, A. Weismann, S. V. Yalunin, and C. Ropers, Tip-enhanced strong-field photoemission, Phys. Rev. Lett. 105, 147601 (2010).

- M. Gulde, R. Bormann, A. Weismann, S. Yalunin, and C. Ropers, Confined electron emission with femtosecond timing: nonlinearity, localization, enhancement, SPIE 7600, 76001I (2010). 


\section{Parts of this work have been presented on the following conferences}

- 19th International Conference on Ultrafast Phenomena, Naha, Japan (2014)

- DPG-Frühjahrstagung, Dresden, Germany (2014)

- New Trends and Faces in Ultrafast Structural Dynamics, Stellenbosch, South Africa (2013)

- Frontiers in Optics / Laser Science, Rochester (NY), USA (2012)

- DPG-Frühjahrstagung, Regensburg, Germany (2012) 


\section{Acknowledgments}

An dieser Stelle möchte ich mich bei all denjenigen bedanken, welche auf die eine oder andere Weise bei dem Entstehungsprozess dieser Dissertation mitgewirkt haben.

Mein spezieller Dank gilt hierbei Claus Ropers, der mich über die vier Jahre hinweg stets ausgezeichnet betreut und unterstützt hat und es mir darüber hinaus ermöglichte, die Arbeit auf vielen internationalen Konferenzen und Workshops vorzustellen. Nicht zuletzt die vielen Wetten (um Pizza) und Bestechungsversuche (mit Pizza) haben für eine spannende und gleichzeitig gelöste Atmosphäre gesorgt, so dass die wissenschaftliche Arbeit immer Spaß gemacht hat.

Ebenso hervorragend wurde ich durch Sascha Schäfer unterstützt, dessen hilfreiche Kommentare und umfangreiches Wissen an so mancher Stelle den Weg durch eine vermeintliche Sackgasse gewiesen haben. Vielen Dank und "High five"!

Tim Salditt und Klaus Sokolowski-Tinten möchte ich meinen Dank dafür aussprechen, sich als Korreferenten für die Arbeit zur Verfügung gestellt zu haben.

Die unkomplizierte ideelle und finanzielle Förderung meiner Promotion durch die Studienstiftung des deutschen Volkes erkenne ich ebenfalls dankend an. Die Unterstützung ermöglichte es mir nicht nur, mich stärker meiner Arbeit zu widmen, auch die Ergebnisse konnte ich immer zeitnah vor einem internationalen Publikum präsentieren.

Simon Schweda, Manisankar Maiti und Gero Storeck möchte ich für die produktive, kreative und stets lustige Zusammenarbeit im Labor danken. Insbesondere das abwechselnde Messen (mit und ohne Messmütze) hat die Arbeit effektiv vorangebracht. Bevor ich Euch kannte, war es einfach cool, einen Kapuzenpulli zu tragen, jetzt verstehe ich endlich, wozu sie wirklich gut sind. 
For the excellent sample preparation and fun hours in the laboratory as well as excellent and often entertaining discussions I would like to thank Manisankar Maiti, Hakki Yu, Alec Wodtke and Simon Schweda: "Sample? You can have it!".

Des Weiteren möchte ich Philipp Kloth dafür meinen Dank aussprechen, dass er mich in die Geheimnisse des STM-Spitzenätzens eingewiesen hat, sowie Philipp Willke, Philipp Ansorg, Thomas Kotzott und Martin Wenderoth für die Messungen am STM.

Holger Stark vom MPI für biophysikalische Chemie möchte ich ganz herzlich danken, dass er sich so viel Zeit für die Probencharakterisierung mit dem Kryo-TEM genommen hat.

Ohne die stets hilfsbereiten Mitarbeiter der Werkstätten des IV. Physikalisches Instituts und des Instituts für Materialphysik sowie der Zentralwerkstatt der Physik hätte das Projekt nie in dieser Form Erfolg haben können. Besonderen Dank möchte ich in diesem Fall Alexander Gehrt sowie Christof Schmidt für die enorme Unterstützung beim Bau des Vakuumsystems aussprechen.

Felix Schenk, Thorsten Hohage und Axel Munk möchte ich danken für die Erstellung der leistungsfähigen FEM Software, mit der die Simulationen in dieser Arbeit gerechnet wurden.

For clarifying discussions about polymer dynamics and their simulation I would like to thank Marcus Müller and Kostas Daoulas. In this view I would also like to mention the productive and fun cooperation with the University of Crete, Greece. Thank you, Vagelis Harmandaris, Tasia Rissanou, Albert "Party" Power, Veronas Petrakis, and Despoina Tzeli, for introducing me to gromacs and not letting me starve while trying to adapt to the Greek lunch and dinner times: "Time is an illusion. Lunchtime doubly so."

Melanie Müller und Alexander Paarmann möchte ich für die Denkanstöße und Diskussionen zu Oberflächenphysik, Veröffentlichungen, afrikanischem und japanischem Essen danken.

Bei der gesamten Arbeitsgruppe Ropers möchte ich mich bedanken für eine tolle und lustige Zeit. Nie werde ich die Übersegelung des Löschteichs der Physik, gemeinsames Badminton, Grillen, Skifahren oder Gokartrennen vergessen. Bleibt so entspannt! 
I would like to thank Daniel Solli not only for proof-reading the thesis at ludicrous speed ("ain't found no more mistakes"), but also for helping me convert all units to the imperial system.

Die Arbeit im gesamten IV. Physikalisches Institut ist geprägt von einer sehr angenehmen und hilfsbereiten Atmosphäre. Den Mitarbeitern des Instituts für Materialphysik möchte ich Dank dafür aussprechen, dass wir so herzlich im Institut aufgenommen und eingegliedert wurden.

Darüber hinaus danke ich meinen Freunden dafür, dass und wie ("autsch") sie es immer geschafft haben, eine Balance zwischen Physik und Freizeit herzustellen. Insbesondere danke ich hier auch dem Sofa, welches mich über lange Jahre so manche späte Nacht noch aufgenommen hat. Und natürlich den Mitbewohnern des Sofas: Hajo, Reiner, Christian, Carla und irgendwie auch Sergey, die zu später Stunde noch Pizza und anspruchsvolle Filme mit mir geteilt haben.

Außerdem bedanke ich mich bei meiner Familie, die mich über die lange Zeit immer unterstützt hat, gerade dann, wenn es mal wieder nicht so ultraschnell lief. Und nicht zuletzt danke ich meiner Julia, die einfach immer für mich da ist, sei es in einem norwegischen Sumpf, auf einem Pass in den Alpen oder einfach nur zu Hause beim Abendessen. 